\title{
Application of Optimal Prediction to Molecular Dynamics
}

\author{
by \\ John Letherman Barber IV \\ B.S. (University of California, Santa Barbara) 1998 \\ M.A. (University of California, Berkeley) 2000 \\ A dissertation submitted in partial satisfaction of the \\ requirements for the degree of \\ Doctor of Philosophy \\ in \\ Physics \\ in the \\ GRADUATE DIVISION \\ of the \\ UNIVERSITY OF CALIFORNIA, BERKELEY
}

\author{
Committee in charge: \\ Professor Alexandre J. Chorin, Co-Chair \\ Professor Edgar Knobloch, Co-Chair \\ Professor Jonathan S. Wurtele \\ Professor Ole H. Hald
}

Fall 2004 
The dissertation of John Letherman Barber IV is approved:

\begin{tabular}{ll}
\hline Co-Chair & Date \\
\hline Co-Chair & Date \\
& \\
\hline & Date \\
\hline & Date
\end{tabular}

University of California, Berkeley

Fall 2004 
Application of Optimal Prediction to Molecular Dynamics

Copyright 2004

by

John Letherman Barber IV 


\author{
Abstract \\ Application of Optimal Prediction to Molecular Dynamics \\ by \\ John Letherman Barber IV \\ Doctor of Philosophy in Physics \\ University of California, Berkeley \\ Professor Alexandre J. Chorin, Co-Chair \\ Professor Edgar Knobloch, Co-Chair
}

Optimal prediction is a general system reduction technique for large sets of differential equations. In this method, which was devised by Chorin, Hald, Kast, Kupferman, and Levy, a projection operator formalism is used to construct a smaller system of equations governing the dynamics of a subset of the original degrees of freedom. This reduced system consists of an effective Hamiltonian dynamics, augmented by an integral memory term and a random noise term.

Molecular dynamics is a method for simulating large systems of interacting fluid particles. In this thesis, I construct a formalism for applying optimal prediction to molecular dynamics, producing reduced systems from which the properties of the original system can be recovered. These reduced systems require significantly less computational time than the original system.

I initially consider first-order optimal prediction, in which the memory and noise terms are neglected. I construct a pair approximation to the renormalized potential, and ignore three-particle and higher interactions. This produces a reduced system that correctly reproduces static properties of the original system, such as energy and pressure, at low-tomoderate densities. However, it fails to capture dynamical quantities, such as autocorrelation functions.

I next derive a short-memory approximation, in which the memory term is represented as a linear frictional force with configuration-dependent coefficients. This allows the use of a Fokker-Planck equation to show that, in this regime, the noise is $\delta$-correlated in time. This linear friction model reproduces not only the static properties of the original system, but also the autocorrelation functions of dynamical variables.

Professor Alexandre J. Chorin

Dissertation Committee Co-Chair

Professor Edgar Knobloch

Dissertation Committee Co-Chair 
To Dove and my mother and father. 


\section{Contents}

List of Figures $\quad$ iv

1 Introduction 1

2 Mathematical Background 5

2.1 Problem Description . . . . . . . . . . . . . . . . 5

2.2 Basic Probability Theory and the Projection Operator . . . . . . . . . 6

2.3 The Mori-Zwanzig Formalism . . . . . . . . . . . . . . . . . . . . 10

2.4 Optimal Prediction for Hamiltonian Systems . . . . . . . . . . . . . 11

3 Molecular Dynamics $\quad 15$

3.1 The Molecular Dynamics Hamiltonian . . . . . . . . . . . . . . . . 15

3.2 Optimal Prediction and the Molecular Dynamics Hamiltonian . . . . . . . . 17

3.3 Thermodynamic Quantities . . . . . . . . . . . . . . . . 17

3.4 Numerical Methods . . . . . . . . . . . . . . . . . . . . . . . . . . 19

3.4.1 The Velocity Verlet Algorithm . . . . . . . . . . . . . . . . . 19

3.4.2 Boundary Conditions and Initial Conditions . . . . . . . . . . . . . . 21

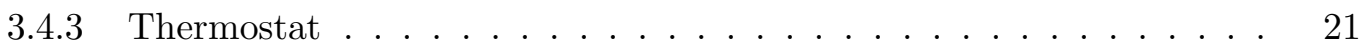

3.4.4 Potential Truncation and Neighbor Lists . . . . . . . . . . . . . . . . 24

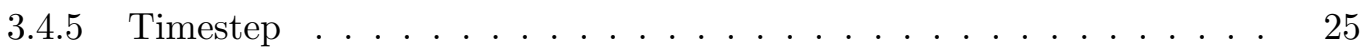

3.4.6 Lennard-Jones Reduced Units . . . . . . . . . . . . . . . . . . . . 26

3.5 Phase Diagram of the Lennard-Jones System . . . . . . . . . . . . . 26

4 First Order Optimal Prediction $\quad 28$

4.1 A Markovian Approximation to Optimal Prediction . . . . . . . . . . . 28

4.2 First Order Optimal Prediction for Molecular Dynamics . . . . . . . . . . . 30

4.2.1 Low-Density Diagrammatic Methods . . . . . . . . . . . . . . . . 31

4.2.2 The General Case: $M$-particle Distribution Functions . . . . . . . . 45

4.2.3 Convergence of the Virial Expansion . . . . . . . . . . . . . . . 47

4.2.4 Calculating the Renormalized Pair Potential . . . . . . . . . . . . . 49

4.3 The Relationship Between the Full and Reduced Systems . . . . . . . . . . 50

4.3 .1 Static Averages . . . . . . . . . . . . . . . . 53

4.3.2 Equilibrium Autocorrelation Functions . . . . . . . . . . . . . 57

4.4 Consequences of Neglecting Many-Particle Interactions . . . . . . . . . . . . 58

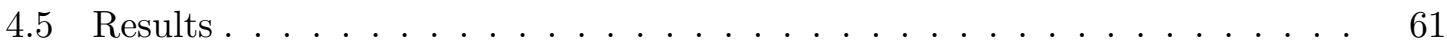

4.5 .1 Full System . . . . . . . . . . . . . . . . . . . 61

4.5 .2 Reduced Systems . . . . . . . . . . . . . . . . . . . . 64 
5 The Linear Friction Approximation $\quad 77$

5.1 Approximating the Memory Kernel . . . . . . . . . . . . . . . . . 77

5.2 Fokker-Planck Equations and the Forcing Term . . . . . . . . . . . . . 81

5.3 Relaxation to Equilibrium . . . . . . . . . . . . . . . . . . . 85

5.4 Approximating the Friction Matrices and Random Forces . . . . . . . . . . 91

5.5 Finding the Optimal Microscopic Timescale . . . . . . . . . . . . . . . . . 103

5.5 .1 Low Density . . . . . . . . . . . . . . . . . 103

5.5 .2 High Density . . . . . . . . . . . . . . . . . 106

5.5.3 Comparison to Optimal Values . . . . . . . . . . . . . . . 107

5.6 Optimal Prediction and Dissipative Particle Dynamics . . . . . . . . . . . 108

5.7 Numerical Tests of the Linear Friction Approximation . . . . . . . . . . . . 112

5.7 .1 Numerical Algorithm . . . . . . . . . . . . . . . . . . . . . . 112

5.7 .2 Results ............................ 114

6 Conclusions $\quad 122$

$\begin{array}{ll}\text { Bibliography } & 125\end{array}$

A Evaluation of the Shear Stress Autocorrelation Function at $t=0 \quad 130$ 


\section{List of Figures}

3.1 The Lennard-Jones Potential _ . . . . . . . . . . . . . . . . . 16

3.2 The truncated Lennard-Jones Potential . . . . . . . . . . . . . 25

3.3 Phase diagram for the Lennard-Jones system . . . . . . . . . . . . . . 27

4.1 Diagrams contributing to the first and second order corrections to the LennardJones potential . . . . . . . . . . . . . . . . . . . 43

4.2 The renormalized Lennard-Jones potential at low densities . . . . . . . . . . 44

4.3 Lower bound on the radius of convergence of the virial series. . . . . . . . . 49

4.4 Radial distribution functions and renormalized potentials derived from the Lennard-Jones potential . . . . . . . . . . . . . . . 51

4.5 Runtime of the full system as a function of density . . . . . . . . . . 62

4.6 Energy per particle of the full system . . . . . . . . . . . . . . 63

4.7 Pressure of the full system . . . . . . . . . . . . . . . 63

4.8 Heat capacity per particle of the full system . . . . . . . . . . . . . . 64

4.9 Cutoff radius of the renormalized pair potential . . . . . . . . . . 65

4.10 Runtimes of the FOOP systems . . . . . . . . . . . . . . 66

4.11 Runtimes of the Galerkin systems . . . . . . . . . . . . . . 66

4.12 Energy calculated via FOOP . . . . . . . . . . . . . . . 68

4.13 Energy calculated via the Galerkin system . . . . . . . . . . . . . . . . 68

4.14 Pressure calculated via FOOP . . . . . . . . . . . . . . . . . 69

4.15 Pressure calculated via the Galerkin system . . . . . . . . . . . . . 69

4.16 Heat Capacity calculated via FOOP . . . . . . . . . . . . . . . 70

4.17 Heat Capacity calculated via the Galerkin system . . . . . . . . . . . . . 70

4.18 Errors in the energy calculated via FOOP . . . . . . . . . . . . . . 72

4.19 Errors in the energy calculated via the Galerkin system . . . . . . . . . . 72

4.20 Errors in the pressure calculated via FOOP . . . . . . . . . . . 73

4.21 Errors in the pressure calculated via the Galerkin system . . . . . . . . . . 73

4.22 Velocity autocorrelation functions calculated via FOOP . . . . . . . . . 75

4.23 Shear stress autocorrelation functions calculated via FOOP . . . . . . . 76

$5.1 \quad \Gamma(\rho)$ for the Lennard-Jones system . . . . . . . . . . . . . . . 95

$5.2 \xi^{\|}(r)$ and $\xi^{\perp}(r)$ for the Lennard-Jones potential . . . . . . . . . . . 96

$5.3 \chi^{(1)}(r)$ and $\chi^{(2)}(r)$ for the Lennard-Jones potential . . . . . . . . . . . . 98

5.4 The set of $(\widehat{\rho}, \widetilde{\rho})$ pairs considered in this thesis . . . . . . . . . . . 100

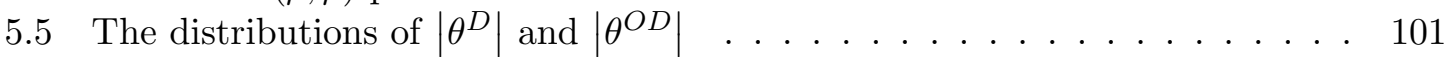

5.6 The distributions of the $A^{i i} \ldots \ldots \ldots \ldots \ldots \ldots \ldots$

5.7 Illustration of the frequency of interparticle encounters . . . . . . . . . . . 104

5.8 The microscopic timescale $\tau^{*}$ as a function of $\tilde{\rho}^{*}$, for various values of $\rho^{*}$. 109 
5.9 Block renormalization of a two-dimensional spin system . . . . . . . . . 112

5.10 Runtimes of the linear friction systems . . . . . . . . . . . . . . 115

5.11 Energy calculated via linear friction . . . . . . . . . . . . . 116

5.12 Pressure calculated via linear friction . . . . . . . . . . . . . . . 116

5.13 Heat Capacity calculated via linear friction . . . . . . . . . . . . . . . 117

5.14 Errors in the energy calculated via linear friction . . . . . . . . . . . 118

5.15 Errors in the pressure calculated via linear friction . . . . . . . . . . . 118

5.16 Velocity autocorrelation functions calculated via linear friction . . . . . . . 120

5.17 Shear stress autocorrelation functions calculated via linear friction . . . . . 121 


\section{Acknowledgements}

I am grateful to the members of my thesis committee for many useful comments and criticisms. I would especially like to thank my advisor Alexandre Chorin for all of his patience, advice, and suggestions, as well as for a careful reading of this thesis.

I would also like to thank all of the people in the mathematics group at Lawrence Berkeley National Laboratory who helped me over the last few years with various aspects of this thesis, and with my understanding of mathematics in general: Panagiotis Stinis, Mayya Tokman, Helen Shvets, Pavel Okunev, Thomas Deschamps, Givon Dror, Carlo Nitsch, Paul Krause, Maria Kourkina, and Christopher Cameron.

In addition, I am grateful to Valerie Heatlie, Anne Takezawa, and Donna Sakima for their help in administrative matters. I greatly appreciate the diligent babysitting assistance and loving encouragement from Patricia Barber, John Barber III, Susan Woeltjen and Lance Woeltjen. Without their help, this thesis would not have been possible. I would also like to thank Sirius Barber-Woeltjen for motivating me to finish this work quickly.

Finally, I would like to thank Dove Woeltjen for her detailed proofreading for clarity and style, as well as for all of her love and support. 


\section{Chapter 1}

\section{Introduction}

The last half century has seen great advances in our understanding of the fluid state. To a large extent, this has been due to the development of powerful computational techniques for the simulation of liquids at the molecular scale. These methods have allowed theoretical results to be tested directly via numerical "experiments" on well-defined models. Early efforts in this direction involved the Monte Carlo simulation of systems of hard spheres or disks [43]. Later, techniques were devised to explore the dynamical properties of many-particle systems by direct numerical integration of the equations of motion of a classical model system $[1,52,65,66,36,37]$. These methods, known collectively as "Molecular Dynamics" (MD) were initially used in the 1950's and 1960's to determine the thermodynamic properties of small systems of particles that corresponded to simple, one component, monatomic fluids and solids. In more recent decades, however, MD has found much wider application. Its use has expanded to include the study of polyatomic fluids, defects in crystals, fracture mechanics, cluster dynamics, and biological macromolecules such as proteins and nucleic acids.

Unfortunately, the range of system sizes accessible via this type of first-principles microscopic simulation severely restricts the utility of MD. For a system of $N$ atoms interacting through a classical pair potential, the amount of time required for a typical MD run scales as $N^{2}$ (or, under certain conditions, as $N$; see Chapter 3), where $N$ is usually $\sim 10^{2}$ to $10^{7}$. This limits the size of MD systems to microscopic length and time scales. However, many of the interesting effects and properties of materials occur in the mesoscopic or macroscopic regimes.

There are several methods that have been devised to overcome this limitation. Advances in parallel computing technology have generated great interest in developing efficient algorithms for performing MD simulations on systems of great size. This brute-force approach has been used to model systems as large as $N \sim 10^{9}$ [13], and has found some use in the study of fracture mechanics in large solids. However, this is still many orders of magnitude smaller than the $\sim 10^{23}$ particles in a macroscopic liquid sample.

A more subtle technique for extending MD to larger scales utilizes the concept of coarse graining, in which the underlying microscopic model is approximated by averaging over small time and length scales, to recover a more slowly-varying, macroscopic model. Research on this type of method has branched in many different directions. A recent, promising implementation of this idea is the technique of "Dissipative Particle Dynamics" (DPD) $[25,16,40,18]$. In DPD, the fundamental elements are not the actual particles of the underlying molecular fluid, but are instead mesoscopic "lumps" of material, each of which 
represents a large number of particles. The various fluid elements interact via a conservative pairwise force, with the addition of momentum-conserving frictional and white noise forces. This model has found some success in reproducing macroscopic-scale hydrodynamic effects, although it lacks a firm theoretical basis.

There are many other coarse-graining techniques that have been developed for various purposes. For example, in the work of Kevrekidis, et. al. [63], a formalism involving "lifting" and "restriction" operators for passing back and forth between microscopic atomistic models and macroscopic continuum models allows the extraction of large-scale data from a small-scale molecular simulation. Along the same lines, Weinan E and collaborators [15] have devised an atomistic-continuum boundary matching scheme, in which certain critical areas of a numerical "sample", such as the vicinity of a fracture, are modelled using a particle-based method, whereas the remainder of the material is modelled using continuum techniques. These types of "multiscale" methods allow the efficient numerical modelling of materials on large spatial and temporal scales, by restricting the application of relatively time-consuming particle-based algorithms to a small fraction of the space or time domain of the full system.

A different implementation of coarse-graining, to be used for determining the properties of large molecules, has been pioneered by Schütte and others [56]. In this approach, instead of studying the exact behavior of a large cluster of particles or a biological macromolecule directly, a finite number of "almost invariant" configurations are identified. The transition probabilities between these states are then calculated by discretizing phase space and performing a Monte-Carlo simulation. Although this severely restricts the amount of information obtained from the system, it allows the determination of many of the macroscopic properties of interest, such as the gross topology of the molecule under study. Other coarse graining methods include lattice techniques [31], and techniques in which systems of polymers are approximated as collections of soft, ellipsoidal particles [46], as well as many others.

The Optimal Prediction (OP) method of Chorin, Hald, Kupferman, and collaborators $[7,8,9,10,11,12,20]$, is a system reduction technique for general differential equations that is closely related to the idea of coarse graining. Given a large system of equations, the degrees of freedom are split into "resolved variables" and "unresolved variables". Under the assumption that there is some prior statistical information about the distribution of the initial conditions in the form of an invariant measure, a smaller set of equations governing the motion of the resolved variables is constructed via the Mori-Zwanzig formalism of nonequilibrium statistical mechanics. This reduced system consists of an effective Hamiltonian dynamics augmented by an integral memory term, as well as a random forcing term.

Molecular dynamics is an excellent candidate for system reduction via OP, due to its underlying Hamiltonian structure, and because the canonical density provides a natural, physically-based measure on the distribution of the coordinates. The most obvious method for applying OP to MD is to divide the particles into "resolved particles" and "unresolved particles", and construct a system of equations that governs the dynamics of the resolved particles alone. This represents a departure from other coarse graining methods, such as those described above, in that the degrees of freedom we retain from the underlying system are not the collective coordinates of many-particle volume elements, but are instead the actual positions and momenta of a representative subset of the original system's molecules. Previous work along these lines has concentrated on the low-temperature limit of a special 
case in one dimension, in which there is a distinct spatial separation maintained between the resolved and unresolved particles [57, 58]. The goal of this thesis is to derive a more general formalism by which OP can be applied to MD at any state point, in any number of dimensions, and without the requirement that the resolved and unresolved particles be kept spatially separated. The resulting method allows the determination of the statistics, as well as the dynamics, of the resolved subset of particles. As we will show in Chapter 4, many of the system's properties of interest can be expressed solely in terms of the coordinates and trajectories of this subset.

This thesis is organized as follows: In Chapter 2, we present some necessary mathematical background from probability theory and introduce a conditional expectation-based projection operator, which allows us to derive the equations of OP via the Mori-Zwanzig formalism. After establishing some notation, we specialize to the case of Hamiltonian systems, which simplifies our expressions somewhat, and provides an elegant framework for what follows.

In Chapter 3, we give an overview of MD. After introducing a very basic model Hamiltonian and describing the interparticle potential, we show in Section 3.2 that OP takes a particularly simple form when applied to this system. We then list several common thermodynamic properties of molecular fluids that will be used in later chapters to test the effectiveness of OP. Finally, we review the popular velocity Verlet algorithm [65, 62], which is commonly used in $\mathrm{MD}$, and which forms the basis for all of the numerical methods used in this thesis. As we will see, various book-keeping methods may be used to reduce the computational cost of an MD simulation from $O\left(N^{2}\right)$ to $O(N)$.

Chapter 4 presents an approximation known as First Order Optimal Prediction (FOOP), in which the memory and random forcing terms are omitted, leaving only the effective Hamiltonian dynamics. The majority of the chapter is concerned with the evaluation of the renormalized Hamiltonian that governs the motion of the resolved particles under this approximation. In Section 4.2.1, we outline how this can be done in the low-density case via a Mayer diagrammatic expansion, where the density of unresolved particles serves as a small parameter. This expansion has much in common with similar diagrammatic methods found in quantum field theory and other areas of physics that involve renormalization. For the general case of arbitrary density, we show in Section 4.2.2 that the renormalized Hamiltonian can be related to the many-particle distribution function familiar from equilibrium statistical mechanics. Section 4.3 gives several examples illustrating how the properties of the original, full system may be recovered from the reduced system. In particular, we derive expressions for the energy, pressure, and heat capacity. In Section 4.5 we test FOOP, and compare the results to those of the full system. We find that FOOP works well at low to moderate densities for determining the static properties of the original system, such as energy or pressure, but fails when dynamical quantities, such as correlation functions, are calculated. This failure is a result of the fact that the memory and forcing terms we have neglected are largely responsible for maintaining the correct temporal correlations in the reduced system.

In order to remedy this, in Chapter 5 we keep the memory and forcing terms, and develop an approximation in which the memory term is modelled as a linear frictional force. In Section 5.2, we show how the statistics of the random forcing are similar to those of $\delta$-correlated white noise, as we derive by considering the Fokker-Planck equation for the distribution of the resolved positions and momenta. Both the coefficent matrices of the friction and the statistics of the white noise turn out to be configuration-dependent. The resulting system of stochastic differential equations has many similarities to the DPD 
approach described above. We next prove in Section 5.3 that this linear friction system necessarily equilibrates to the canonical distribution of the reduced system. The results from this model are comparable to those of FOOP for the calculation of static properties. The linear friction model is also far better at calculating dynamical quantities, particularly velocity autocorrelation functions, although, like FOOP, it breaks down at high densities.

Both of these methods, FOOP and linear friction, require that significant approximations be made to the essentially exact equations of optimal prediction from which they are derived. This inevitably results in appreciable errors in their determination of some of the properties of the fluids they describe, particularly at high densities. Nevertheless, these techniques may serve as the basis for future implementations of molecular dynamics system reduction. 


\section{Chapter 2}

\section{Mathematical Background}

We begin with a general outline of the problem to be solved, as well as a few results and definitions from probability theory. We then derive the primary equation of OP via the Mori-Zwanzig formalism, which is discussed in the context of Hamiltonian dynamical systems.

\subsection{Problem Description}

As our starting point, we consider the system

$$
\begin{aligned}
\frac{d}{d t} \varphi(z, t) & =R(\varphi(z, t)) \\
\varphi(z, 0) & =z .
\end{aligned}
$$

Here, $\varphi, z$, and $R$ are all $N$-dimensional vectors, with components $\varphi_{i}, z_{i}$, and $R_{i}$. Equation (2.1) is an autonomous set of ordinary differential equations (ODEs), where $R$ is generally

taken to be nonlinear. The dependence of $\varphi$ on the initial condition $z$ is explicitly included here because of its importance for later results.

Following Chorin [6], the nonlinear system of ODEs (2.1) can be recast as a linear partial differential equation (PDE). Consider the function $u(z, t)$, which satisfies

$$
\begin{aligned}
\partial_{t} u(z, t) & =L u(z, t) \\
u(z, 0) & =g(z),
\end{aligned}
$$

where

$$
L=\sum_{i=1}^{N} R_{i}(z) \partial_{z_{i}}
$$

is the Liouville operator, and $g(z)$ is an arbitrary initial profile. The solution to (2.2) can be written formally as

$$
u(z, t)=e^{t L} g(z)
$$

where we have used semigroup notation [49] for the evolution operator $e^{t L}$ generated by $L$. The following identities hold for $e^{t L}$ :

$$
\begin{aligned}
e^{0 L} & =I, \\
L e^{t L} & =e^{t L} L, \\
e^{t L} h(z) & =h\left(e^{t L} z\right) .
\end{aligned}
$$


In $(2.5 \mathrm{a}), I$ is the identity operator, and in $(2.5 \mathrm{c}) h(z)$ is an arbitrary function of $z$.

In addition to the formal expression (2.4), the solution to (2.2) can also be written explicitly in terms of the solution to (2.1) as

$$
u(z, t)=g(\varphi(z, t))
$$

(A proof of this, as well as proofs of the identities above, can be found in [6].) The two systems (2.1) and (2.2) are equivalent in that knowledge of the solution to one for all initial conditions immediately yields the solution to the other. If we set $g(z)=z_{i}$ in $(2.2)$, we find that $u(z, t)=\varphi_{i}(z, t)$. Combining this with (2.4) yields

$$
\varphi_{i}(z, t)=e^{t L} z_{i}
$$

which, together with (2.5b), allows us to write the system (2.1) as

$$
\begin{aligned}
\frac{d}{d t} \varphi(z, t) & =e^{t L} L z \\
\varphi(z, 0) & =z .
\end{aligned}
$$

We now make the assumption that (2.6) is too difficult to treat by traditional methods, either because it is too large a system, or because we lack complete information about the initial condition $z$. Our goal is to construct a smaller $M$-dimensional system, with $M<N$. Ideally, the statistical and dynamical properties of this smaller system should mirror those of (2.6).

The initial condition $z$ is partitioned into two parts, so that $z=\{\widehat{z}, \widetilde{z}\}$, where $\widehat{z}=\left\{z_{1}, \ldots, z_{M}\right\}$ and $\widetilde{z}=\left\{z_{M+1}, \ldots, z_{N}\right\}$. We similarly partition $\varphi=\{\widehat{\varphi}, \widetilde{\varphi}\}$, and $R=\{\widehat{R}, \widetilde{R}\}$. It is assumed that $\widehat{z}$ is known, whereas $\widetilde{z}$ is unknown. We therefore seek to obtain an expression for the evolution of $\widehat{\varphi}$ alone, since $\widehat{\varphi}(0)=\widehat{z}$ is known. The $\widehat{\varphi}_{i}$ are called the resolved variables, and the $\widetilde{\varphi}_{i}$ are called the unresolved variables.

If $\widehat{z}$ is known, but we have no information of any kind about $\widetilde{z}$, then little can be done to achieve this goal. Therefore the additional assumption is made that we have some statistical knowledge about the distribution of the initial conditions in the form of a probability density function $f$ on $z$. Before outlining how the existence of such a density helps us to overcome the obstacles inherent in a large nonlinear system such as (2.6), we must make a brief digression to present some results and definitions from probability theory.

\subsection{Basic Probability Theory and the Projection Operator}

We begin with a vector-valued random variable $Z=\left(Z_{1}, \ldots, Z_{N}\right)$. The set of all possible values of $Z$ is $\Omega$, which is known as the sample space. An event is defined to be a subset of $\Omega$, and $\mathscr{B}$ is the set of events we can observe, which is assumed to be a $\sigma$-algebra, i.e. it satisfies the following conditions:

(1) $\emptyset \in \mathscr{B}$, where $\emptyset$ is the empty set.

(2) $\Omega \in \mathscr{B}$.

(3) If $A \in \mathscr{B}$, then $A^{c} \in \mathscr{B}$, where $A^{c} \equiv\{z \in \Omega \mid z \notin A\}$ is the complement of $A$.

(4) If $A_{1}, A_{2}, \ldots$ is a sequence of sets in $\mathscr{B}$, and $A=\bigcup_{n=1}^{\infty} A_{n}$, then $A \in \mathscr{B}$. 
(Note that (1) follows from (2) and (3).) A function $P: \mathscr{B} \rightarrow \mathbb{R}$ is said to be a probability measure if

(1) $P(\emptyset)=0$,

(2) $0 \leq P(A) \leq 1$ for all $A \in \mathscr{B}$,

(3) $P(\Omega)=1$,

(4) For any sequence $A_{1}, A_{2}, \ldots$ of sets in $\mathscr{B}$ which satisfy $A_{i} \cap A_{j}=0$ for $i \neq j$, we have $P\left(\bigcup_{n=1}^{\infty} A_{n}\right)=\sum_{n=1}^{\infty} P\left(A_{n}\right)$.

$P$ assigns to each event in $\mathscr{B}$ a probability in $[0,1]$, and the triplet $(\Omega, \mathscr{B}, P)$ is called a probability space.

Example: If we take our random variable $Z$ to be the number that results from rolling a die, then $\Omega=\{1,2,3,4,5,6\}$. The set of events $\mathscr{B}$, however, depends on what we choose to define as an "event". We may, for example, choose to include as events every possible subset of $\Omega$, in which case $\mathscr{B}$ has $2^{6}=64$ members, including $\emptyset$ and $\Omega$ itself. On the other hand, we may decide to recognize only the two events " $Z$ is even" and " $Z$ is odd", in which case $\mathscr{B}=\{\emptyset,\{2,4,6\},\{1,3,5\}, \Omega\}$. In that case, the probability measure would satisfy $P(\emptyset)=0$, $P(\Omega)=1$, and $P(\{2,4,6\})=P(\{1,3,5\})=1 / 2$.

Information about the probability measure associated with a continuous random variable $Z$ is usually given in the form of a distribution function $F(z)$, which is related to $P$ via

$$
P\left(\left\{Z \in \Omega \mid Z_{1} \leq z_{1}, \ldots, Z_{N} \leq z_{N}\right\}\right)=F(z) .
$$

The random variable $Z$ is then said to have the probability density function (PDF) $f$ if

$$
F(z)=\int_{-\infty}^{z_{1}} d y_{1} \ldots \int_{-\infty}^{z_{N}} d y_{N} f(y)
$$

$f$ obeys the following axioms:

(1) $f(z) \geq 0$ for all $z \in \Omega$

(2) $f$ is integrable on all elements $A \in \mathscr{B}$

(3) $\int_{\Omega} d z f(z)=1$

(4) $\int_{A} d z f(z)$ is the probability that $z \in A \subseteq \Omega$

Given $f$, we can compute the mean, or expectation value, of a function $g$ of $z$ as

$$
E[g] \equiv \int_{\Omega} d z g(z) f(z)
$$

which yields a number independent of $z$.

In our case, the random vector $z$ of initial conditions to equation (2.1) is partially specified, because we have a value for $\widehat{z}$, the vector of the first $M$ components of $z$. We can use this partial information to construct a conditional probability density function on $\widetilde{z}$ alone. This conditional PDF is denoted by $f(\widetilde{z} \mid \widehat{z})$, and is a function of $\widetilde{z}$, parametrized by 
the known value of $\widehat{z}$. If $\widetilde{\Omega}$ is the sample space of $\widetilde{z}$ alone, then $f(\widetilde{z} \mid \widehat{z})$ is given in terms of the full density $f$ by

$$
f(\widetilde{z} \mid \widehat{z})=\frac{f(z)}{\int_{\widetilde{\Omega}} d \widetilde{z} f(z)}
$$

where, in general, $\widetilde{\Omega}$ depends on $\widehat{z}$. Note that (2.8) implies that $f(\widetilde{z} \mid \widehat{z})$ satisfies a similar set of axioms as $f$ :

(1) $f(\widetilde{z} \mid \widehat{z}) \geq 0$ for all $\widetilde{z} \in \widetilde{\Omega}, \widehat{z} \in \widehat{\Omega}$

(2) $f(\widetilde{z} \mid \widehat{z})$ is integrable on all elements $A \in \widetilde{\mathscr{B}}$, where $\widetilde{\mathscr{B}}$ is a $\sigma$-algebra of subsets of $\widetilde{\Omega}$

(3) $\int_{\widetilde{\Omega}} d \widetilde{z} f(\widetilde{z} \mid \widehat{z})=1$ for all $\widehat{z} \in \widehat{\Omega}$

(4) $\int_{A} d \widetilde{z} f(\widetilde{z} \mid \widehat{z})$ is the probability that $\widetilde{z} \in A \subseteq \widetilde{\Omega}$, given $\widehat{z}$.

By analogy with (2.7), we can use the conditional density (2.8) to define the average value of a function $g$ of $z$, constrained by the known value of $\widehat{z}$. This is known as the conditional expectation value of $g(z)$, and is given by

$$
\begin{aligned}
E[g(z) \mid \widehat{z}] & \equiv \int_{\widetilde{\Omega}} d \widetilde{z} g(z) f(\widetilde{z} \mid \widehat{z}) \\
& =\frac{\int_{\widetilde{\Omega}} d \widetilde{z} g(z) f(z)}{\int_{\widetilde{\Omega}} d \widetilde{z} f(z)} .
\end{aligned}
$$

Several properties of the conditional expectation are listed below. Here $g(z)$ and $h(z)$ are arbitrary functions of $z$ for which $E[g], E[h], E\left[g^{2}\right]$, and $E\left[h^{2}\right]$ exist; $w(\widehat{z})$ is an arbitrary function of $\widehat{z}$ for which $E[w]$ and $E\left[w^{2}\right]$ exist; and $\alpha$ and $\beta$ are constants.

(1) $E[g(z) \mid \widehat{z}]$ is a function of $\widehat{z}$ alone.

(2) $E[w(\widehat{z}) g(z) \mid \widehat{z}]=w(\widehat{z}) E[g(z) \mid \widehat{z}]$

(3) $E[E[g(z) \mid \widehat{z}]]=E[g(z)]$

(4) $E[\alpha g(z)+\beta h(z) \mid \widehat{z}]=\alpha E[g(z) \mid \widehat{z}]+\beta E[h(z) \mid \widehat{z}]$

(5) $E\left[(g(z)-E[g(z) \mid \widehat{z}])^{2}\right] \leq E\left[(g(z)-w(\widehat{z}))^{2}\right]$

Properties (1)-(4) follow simply from the definition (2.9) of conditional expectation. Property (5) is a statement of the fact that $E[g(z) \mid \widehat{z}]$ is the best approximation of $g(z)$ by a 
function of $\widehat{z}$, and can be proven as follows:

$$
\begin{aligned}
E\left[(g(z)-w(\widehat{z}))^{2}\right]= & E\left[(g(z)-E[g(z) \mid \widehat{z}]+E[g(z) \mid \widehat{z}]-w(\widehat{z}))^{2}\right] \\
=E & {\left[(g(z)-E[g(z) \mid \widehat{z}])^{2}\right]+E\left[(w(\widehat{z})-E[g(z) \mid \widehat{z}])^{2}\right] } \\
& -2 E[(g(z)-E[g(z) \mid \widehat{z}])(w(\widehat{z})-E[g(z) \mid \widehat{z}])] \\
= & E\left[(g(z)-E[g(z) \mid \widehat{z}])^{2}\right]+E\left[(w(\widehat{z})-E[g(z) \mid \widehat{z}])^{2}\right] \\
\geq E & {\left[(g(z)-E[g(z) \mid \widehat{z}])^{2}\right], }
\end{aligned}
$$

where in the third equality we have used the fact that

$$
\begin{aligned}
E[(g(z)- & E[g(z) \mid \widehat{z}])(w(\widehat{z})-E[g(z) \mid \widehat{z}])] \\
& =E[E[(g(z)-E[g(z) \mid \widehat{z}])(w(\widehat{z})-E[g(z) \mid \widehat{z}]) \mid \widehat{z}]] \quad \text { By (3) } \\
& =E[(w(\widehat{z})-E[g(z) \mid \widehat{z}]) \underbrace{E[(g(z)-E[g(z) \mid \widehat{z}]) \mid \widehat{z}]}_{=0} \quad \text { By (2) } \\
& =0 .
\end{aligned}
$$

We now define a projection operator $\mathbb{P}$ that acts on the space of functions of $z$,

$$
\mathbb{P}\{g(z)\}=(\mathbb{P} g)(\widehat{z}) \equiv E[g(z) \mid \widehat{z}] .
$$

$\mathbb{P}$ is the orthogonal projection onto the space of functions of $\widehat{z}$, and is the same projection as the one described in $[7,8,9,10,11]$. We further define the operator $\mathbb{Q}=I-\mathbb{P} . \mathbb{P}$ and $\mathbb{Q}$ satisfy the operator identities

$$
\begin{aligned}
\mathbb{P}^{2} & =\mathbb{P} \\
\mathbb{Q}^{2} & =\mathbb{Q} \\
\mathbb{P} \mathbb{Q} & =\mathbb{Q P}=0 .
\end{aligned}
$$

Here (2.11c) is an expression of the fact that $\mathbb{Q}$ is the orthogonal projection onto the null space of $\mathbb{P}$.

It is worth noting that there are several other projections commonly in use. For example, if we take the inner product of two functions $g_{1}(z)$ and $g_{2}(z)$ to be $\left(g_{1}, g_{2}\right)=E\left[g_{1} g_{2}\right]$, then we may define the linear projection $\mathbb{P}^{L}$, where

$$
\mathbb{P}^{L}\{g(z)\}=\left(\mathbb{P}^{L} g\right)(\widehat{z}) \equiv \sum_{i, j=1}^{M}\left(a^{-1}\right)_{i j}\left(g(z), \widehat{z}_{i}\right) \widehat{z}_{j}
$$

Here $a^{-1}$ is the inverse of the $M \times M$ matrix $a$, which has entries $a_{i j}=\left(\widehat{z}_{i}, \widehat{z}_{j}\right)$. The linear projection is used extensively in the nonequilibrium statistical mechanics literature $[17,19]$. 
More generally, if we pick a set of $M$ functions $h^{\nu}(\widehat{z})$, which are orthonormal in the sense that $\left(h^{\nu}, h^{\mu}\right)=\delta_{\nu \mu}$, then the finite-rank projection $\mathbb{P}^{F R}$ is given by

$$
\mathbb{P}^{F R}\{g(z)\}=\left(\mathbb{P}^{F R} g\right)(\widehat{z}) \equiv \sum_{\nu=1}^{M}\left(g(z), h^{\nu}\right) h^{\nu} .
$$

If the set of functions $h^{\nu}(\widehat{z})$ spans $\widehat{\Omega}$ as $M$ becomes large, then in this limit $\mathbb{P}^{F R}$ approximates the projection $\mathbb{P}$ defined by (2.10).

The projections $\mathbb{P}^{L}$ and $\mathbb{P}^{F R}$ are both useful in certain types of problems. However, for reasons that will be made clear in Chapter 4, in the context of molecular dynamics only the projection $\mathbb{P}$ is appropriate.

\subsection{The Mori-Zwanzig Formalism}

Following the Mori-Zwanzig procedure $[9,17,44,68,69]$, we use the fact that $\mathbb{P}+\mathbb{Q}=I$ to split the Liouville operator into two parts: $L=\mathbb{P} L+\mathbb{Q} L$. This allows us to write the system (2.6) as

$$
\frac{d}{d t} \varphi(z, t)=e^{t L}(\mathbb{P} L+\mathbb{Q} L) z=e^{t L} \mathbb{P} L z+e^{t L} \mathbb{Q} L z,
$$

which describes the evolution of the full $N$-dimensional vector $\varphi$. Since we are only interested in the evolution of $\widehat{\varphi}$, we choose to focus on the $i^{t h}$ component of (2.14), where $1 \leq i \leq M$. Keeping in mind that $\varphi_{i}=\widehat{\varphi}_{i}$ and $z_{i}=\widehat{z}_{i}$ for $i$ in this range, we then arrive at

$$
\frac{d}{d t} \widehat{\varphi}_{i}(z, t)=e^{t L} \mathbb{P} L \widehat{z}_{i}+e^{t L} \mathbb{Q} L \widehat{z}_{i} .
$$

The second term in $(2.15)$ can be split further. Define $w_{i}(z, t)$ to be the solution to

$$
\begin{aligned}
\partial_{t} w_{i}(z, t) & =\mathbb{Q} L w_{i}(z, t) \\
w_{i}(z, 0) & =\mathbb{Q} L \widehat{z}_{i},
\end{aligned}
$$

which is known as the orthogonal dynamics equation. The formal solution to (2.16) can be written using semigroup notation as $w_{i}(z, t)=e^{t \mathbb{Q} L} \mathbb{Q} L \widehat{z}_{i}$. A proof of the existence of weak solutions to (2.16) can be found in [20].

An important property of $w_{i}(z, t)$ is that $\mathbb{P} w_{i}(z, t)=0$ for all $t$. This can be seen by noting that $\mathbb{P} w_{i}(z, 0)=\mathbb{P} \mathbb{Q} L \widehat{z}_{i}=0$, by $(2.11 \mathrm{c})$. Furthermore, the time derivative of $\mathbb{P} w_{i}(z, t)$ is

$$
\begin{aligned}
\frac{d}{d t} \mathbb{P} w_{i}(z, t) & =\mathbb{P} \frac{d}{d t} w_{i}(z, t) \\
& =\mathbb{P} \mathbb{Q} L w_{i}(z, t) \\
& =0,
\end{aligned}
$$

which implies that $\mathbb{P} w_{i}(z, t)=0$ for all $t$. (The commutativity of $\mathbb{P}$ and $\frac{d}{d t}$ follows from the definition of $\mathbb{P}$.) The dynamics of $w_{i}(z, t)$ therefore lie entirely within the null space of $\mathbb{P}$.

The evolution operators $e^{t L}$ and $e^{t \mathbb{Q} L}$ are related via the Duhamel/Dyson identity [14, 17]:

$$
e^{t L}=\int_{0}^{t} d s e^{(t-s) L} \mathbb{P} L e^{s \mathbb{Q} L}+e^{t \mathbb{Q} L}
$$


Substituting this expression for $e^{t L}$ into the second term of (2.15) yields

$$
\frac{d}{d t} \widehat{\varphi}_{i}(z, t)=e^{t L} \mathbb{P} L \widehat{z}_{i}+\int_{0}^{t} d s e^{(t-s) L} \mathbb{P} L e^{s \mathbb{Q} L} \mathbb{Q} L \widehat{z}_{i}+e^{t \mathbb{Q} L} \mathbb{Q} L \widehat{z}_{i}
$$

If we now define

$$
\begin{aligned}
\mathscr{R}_{i}(\widehat{z}) & =\mathbb{P} L \widehat{z}_{i} \\
\mathscr{K}_{i}(\widehat{z}, t) & =\mathbb{P} L e^{t \mathbb{Q} L} \mathbb{Q} L \widehat{z}_{i} \\
\mathscr{F}_{i}(z, t) & =e^{t \mathbb{Q} L} \mathbb{Q} L \widehat{z}_{i},
\end{aligned}
$$

then we finally arrive at

$$
\frac{d}{d t} \widehat{\varphi}_{i}(z, t)=e^{t L} \mathscr{R}_{i}(\widehat{z})+\int_{0}^{t} d s e^{(t-s) L} \mathscr{K}_{i}(\widehat{z}, s)+\mathscr{F}_{i}(z, t) .
$$

Equation (2.17) is an example of a generalized Langevin equation, and is the primary equation of OP. We refer to (2.17) as the reduced system, whereas (2.1) is the full system. Note that no approximations have been made in deriving (2.17).

The three terms on the right hand side of (2.17) are conventionally understood as follows. The first term, $e^{t L} \mathscr{R}_{i}(\widehat{z})=\mathscr{R}_{i}(\widehat{\varphi}(z, t))$, depends only on the value of $\widehat{\varphi}$ at time $t$, and is a Markovian contribution to the dynamics of $\widehat{\varphi}$. The second term depends upon the entire history of $\widehat{\varphi}(z, s)$ for $0 \leq s \leq t$, and thus represents a memory, with memory kernel $e^{(t-s) L} \mathscr{K}_{i}(\widehat{z}, s)=\mathscr{K}_{i}\left(\widehat{\varphi}_{i}(z, t-s), s\right)$. The last term, $\mathscr{F}_{i}(z, t)$, is the same as $w_{i}(z, t)$ described by equation $(2.16)$, and so $\mathbb{P}_{F_{i}}(z, t)=0$ for all $t$. For this reason, $\mathscr{F}_{i}(z, t)$ is usually interpreted as random noise with zero mean. Notice that $\mathscr{K}_{i}(\widehat{z}, t)=\mathbb{P} L \mathscr{F}_{i}(z, t)$. Such expressions, which relate the memory kernel to the noise, are called fluctuation-dissipation theorems.

In previous work on OP $[7,8,9]$, equation $(2.17)$ was multiplied on the left one final time by $\mathbb{P}$. This eliminates the noise term, since $\mathbb{P} \mathscr{F}_{i}=0$, and yields

$$
\frac{d}{d t} \mathbb{P} \widehat{\varphi}_{i}(z, t)=\mathbb{P} \mathscr{R}_{i}\left(\widehat{\varphi}_{i}(z, t)\right)+\int_{0}^{t} d s \mathbb{P} \mathscr{K}_{i}(\widehat{\varphi}(z, t-s), s),
$$

which is an evolution equation not for $\widehat{\varphi}(z, t)$, but for $\mathbb{P} \widehat{\varphi}(z, t)$. $\mathbb{P} \widehat{\varphi}(z, t)$ represents an average over all solutions with respect to $\widetilde{z}$, while $\widehat{z}$ remains fixed. Since (2.1) is typically nonlinear, the solution will eventually "forget" about our partial knowledge of the initial conditions [27]. Therefore, for the types of systems studied previously, $\mathbb{P} \widehat{\varphi}(z, t)$ converges for large $t$ to a constant value equal to the mean of $\widehat{z}$ under the full density $f$. However, convergence of the system's coordinates to constant values is not consistent with the qualitative behavior of the many-body molecular dynamics systems we will be studying, in which thermal excitations should persist even after the system has come to thermodynamic equilibrium. Therefore we shall work directly with (2.17), rather than with its projected counterpart (2.18).

\subsection{Optimal Prediction for Hamiltonian Systems}

If $(2.1)$ is a Hamiltonian system, with Hamiltonian $H(q, p)$, then it is convenient to make a few cosmetic changes in notation. Let there be $N$ positions $q_{i}$ and $N$ momenta $p_{i}$, each of which is a $d$-dimensional vector. We define $q=\left\{q_{1}, q_{2}, \ldots, q_{N}\right\}$ and $p=\left\{p_{1}, p_{2}, \ldots, p_{N}\right\}$ 
to be the $N d$-dimensional vectors of the positions and momenta, respectively. The set of ODEs generated by $H(q, p)$ is

$$
\begin{aligned}
\frac{d}{d t} q_{i} & =\nabla_{p_{i}} H(q, p) \\
\frac{d}{d t} p_{i} & =-\nabla_{q_{i}} H(q, p) \\
q_{i}(0) & =x_{i} \\
p_{i}(0) & =y_{i} .
\end{aligned}
$$

(Note that the " $\nabla$ " notation is used because the $q_{i}$ and $p_{i}$ are $d$-dimensional vectors.)

We abandon the more general variables $\varphi$ and $z$ in favor of $q, p, x$, and $y$, which are split into $M$ resolved variables and $N-M$ unresolved variables as follows:

$$
\begin{array}{ll}
\widehat{q}=\left\{q_{1}, \ldots, q_{M}\right\} & \widetilde{q}=\left\{q_{M+1}, \ldots, q_{N}\right\} \\
\widehat{p}=\left\{p_{1}, \ldots, p_{M}\right\} & \widetilde{p}=\left\{p_{M+1}, \ldots, p_{N}\right\} \\
\widehat{x}=\left\{x_{1}, \ldots, x_{M}\right\} & \widetilde{x}=\left\{x_{M+1}, \ldots, x_{N}\right\} \\
\widehat{y}=\left\{y_{1}, \ldots, y_{M}\right\} & \widetilde{y}=\left\{y_{M+1}, \ldots, y_{N}\right\} .
\end{array}
$$

Here $\widehat{q}, \widehat{p}, \widehat{x}$, and $\widehat{y}$ are $M d$-dimensional, whereas $\widetilde{q}, \widetilde{p}, \widetilde{x}$, and $\widetilde{y}$ are $(N-M) d$-dimensional. Note that if $q_{i}$ is resolved, then so is its corresponding conjugate momentum $p_{i}$.

In terms of this new notation, (2.17) becomes the pair of equations

$$
\begin{aligned}
\frac{d}{d t} \widehat{q}_{i} & =e^{t L} \mathscr{R}_{i}^{(q)}(\widehat{x}, \widehat{y})+\int_{0}^{t} d s e^{(t-s) L} \mathscr{K}_{i}^{(q)}(\widehat{x}, \widehat{y}, s)+\mathscr{F}_{i}^{(q)}(x, y, t) \\
\frac{d}{d t} \widehat{p}_{i} & =e^{t L} \mathscr{R}_{i}^{(p)}(\widehat{x}, \widehat{y})+\int_{0}^{t} d s e^{(t-s) L} \mathscr{K}_{i}^{(p)}(\widehat{x}, \widehat{y}, s)+\mathscr{F}_{i}^{(p)}(x, y, t),
\end{aligned}
$$

where

$$
\begin{aligned}
\mathscr{R}_{i}^{(q)}(\widehat{x}, \widehat{y}) & =\mathbb{P} L \widehat{x}_{i} & \mathscr{R}_{i}^{(p)}(\widehat{x}, \widehat{y}) & =\mathbb{P} L \widehat{y}_{i} \\
\mathscr{K}_{i}^{(q)}(\widehat{x}, \widehat{y}, t) & =\mathbb{P} L e^{t \mathbb{Q} L} \mathbb{Q} L \widehat{x}_{i} & \mathscr{K}_{i}^{(p)}(\widehat{x}, \widehat{y}, t) & =\mathbb{P} L e^{t \mathbb{Q} L} \mathbb{Q} L \widehat{y}_{i} \\
\mathscr{F}_{i}^{(q)}(x, y, t) & =e^{t \mathbb{Q} L} \mathbb{Q} L \widehat{x}_{i} & \mathscr{F}_{i}^{(p)}(x, y, t) & =e^{t \mathbb{Q} L} \mathbb{Q} L \widehat{y}_{i} .
\end{aligned}
$$

Here the Liouvillian $L$ is

$$
L=\sum_{i=1}^{N}\left\{\left(\nabla_{y_{i}} H(x, y)\right) \cdot \nabla_{x_{i}}-\left(\nabla_{x_{i}} H(x, y)\right) \cdot \nabla_{y_{i}}\right\} .
$$

In section 2.1, we introduced $f$, a probability density on the vector of initial conditions. We did not, however, explain where $f$ comes from. In fact, the form of $f$ is unspecified, and must either be determined from experimental data or deduced from the physics of the problem at hand. Let the probability density of the positions and momenta of the system (2.19) at time $t$ be denoted by $f(x, y, t)$, with $f(x, y, 0)=f_{0}(x, y)$. Then $f(x, y, t)$ satisfies the Liouville equation [17]

$$
\partial_{t} f(x, y, t)=-L f(x, y, t)
$$


where $L$ is as in (2.22). The formal solution to $(2.23)$ is

$$
\begin{aligned}
f(x, y, t) & =e^{-t L} f_{0}(x, y) \\
& =f_{0}\left(e^{-t L} x, e^{-t L} y\right) \\
& =f_{0}(q(x, y,-t), p(x, y,-t)),
\end{aligned}
$$

where $q(x, y,-t)$ and $p(x, y,-t)$ are the solutions to $(2.19)$ at time $-t$. If $A(q(x, y, t), p(x, y, t))$ is some dynamical variable, then its average at time $t$ is

$$
\begin{array}{rlrl}
\langle A\rangle(t) & =\int d x \int d y A(q(x, y, t), p(x, y, t)) f_{0}(x, y) & & \text { (Heisenberg representation) } \\
& =\int d x \int d y f_{0}(x, y) e^{t L} A(x, y) & \\
& =\int d x \int d y A(x, y) e^{-t L} f_{0}(x, y) & \\
& =\int d x \int d y A(x, y) f(x, y, t) . & & \text { (Schroedinger representation) }
\end{array}
$$

In the third equality above, we have used the fact the propagator $e^{-t L}$ is the adjoint of the propagator $e^{t L}$ (see [17]).

An invariant density $f^{*}(x, y)$ satisfies

$$
e^{t L} f^{*}(x, y)=f^{*}(q(x, y,-t), p(x, y,-t))=f^{*}(x, y),
$$

i.e. $f^{*}(x, y)$ is a stationary solution to the Liouville equation. In that case, the average value of the dynamical variable $A$ becomes time-independent: $\langle A\rangle(t)=\langle A\rangle(0)$. An invariant density would therefore be a convenient choice for the $f$ of section 2.1.

In the context of Hamiltonian systems, an excellent candidate for $f$ is the canonical density

$$
f_{c}(x, y)=\frac{1}{Z} e^{-\beta H(x, y)},
$$

where $\beta=1 / k_{B} T, k_{B}$ is Boltzmann's constant, $T$ is the temperature, and

$$
Z=\int d x \int d y e^{-\beta H(x, y)}
$$

is a normalizing factor. The canonical density $f_{c}$ is the equilibrium density for a Hamiltonian system in thermal contact with a heat bath at temperature $\mathrm{T}$, and thus accurately describes the statistics of many real-world systems [48]. It also has the advantage of being an invariant density, which follows from the fact that $L H=0$.

Note that $f_{c}$ is not the only commonly used invariant density for Hamiltonian systems. There are several others, including, for example, the microcanonical distribution

$$
f_{m}(x, y)=\frac{1}{S(E)} \delta(H(x, y)-E)
$$

where $S(E)$ is a normalizing factor, $E$ is the (fixed) energy of the system, and $\delta$ is the Dirac $\delta$-function. $f_{m}$ describes the distribution of coordinates and momenta in an isolated system of constant energy. In reality, however, complete thermodynamic isolation can be realized 
as an approximation at best, as real systems always have some external coupling. For this reason, and for the reasons listed above, we shall use $f_{c}$ exclusively as the PDF of initial conditions throughout this work. This choice follows the example of previous literature on OP $[7,8,9,10,11,12,27,57,58]$. 


\section{Chapter 3}

\section{Molecular Dynamics}

We introduce a Hamiltonian $H$ that governs the dynamics of a simple molecular fluid. After a discussion of several assumptions about the form of $H$, we describe a numerical method for simulating the resulting system. Lastly, we outline the application of OP to our Hamiltonian.

\subsection{The Molecular Dynamics Hamiltonian}

We consider a classical system consisting of $N$ identical, point particles of mass $m$, moving in $d$ dimensions. The particle positions $q_{i}$ are confined within a bounded, simply-connected region $\Omega \subset \mathbb{R}^{d}$. The momenta are $p_{i} \in \mathbb{R}^{d}$. We define $V=|\Omega|$ to be the volume. Throughout this thesis, we will work in the thermodynamic limit, i.e. all results will ultimately be interpreted in the limit $N, V \rightarrow \infty$, while the number density $\rho=N / V$ remains constant ${ }^{1}$.

The Hamiltonian governing the system is:

$$
H(q, p)=\sum_{i=1}^{N} \frac{\left|p_{i}\right|^{2}}{2 m}+\phi(q),
$$

where the potential energy $\phi(q)$ is assumed to be a sum of radially symmetric pair interactions:

$$
\phi(q)=\sum_{i<j}^{N} U\left(\left|q_{i}-q_{j}\right|\right) .
$$

The Hamiltonian (3.1) describes the simplest possible classical system of interacting particles. The assumption of a pairwise potential is an approximation. More realistic $N$-body Hamiltonians may have three-particle, four-particle, or higher interactions. Furthermore, we have neglected quantum effects, as well as the particles' internal degrees of freedom. In fact, (3.1) and (3.2) were used in the earliest versions of MD to be studied numerically $[1,36,37,52,65,66]$. More accurate models have since been developed that take into account many-particle effects and the internal structure of the molecules [3]. These will not be treated here.

\footnotetext{
${ }^{1}$ It is traditional in computational MD for " $\rho$ " to represent number density, rather than mass density, as it typically does in physics.
} 


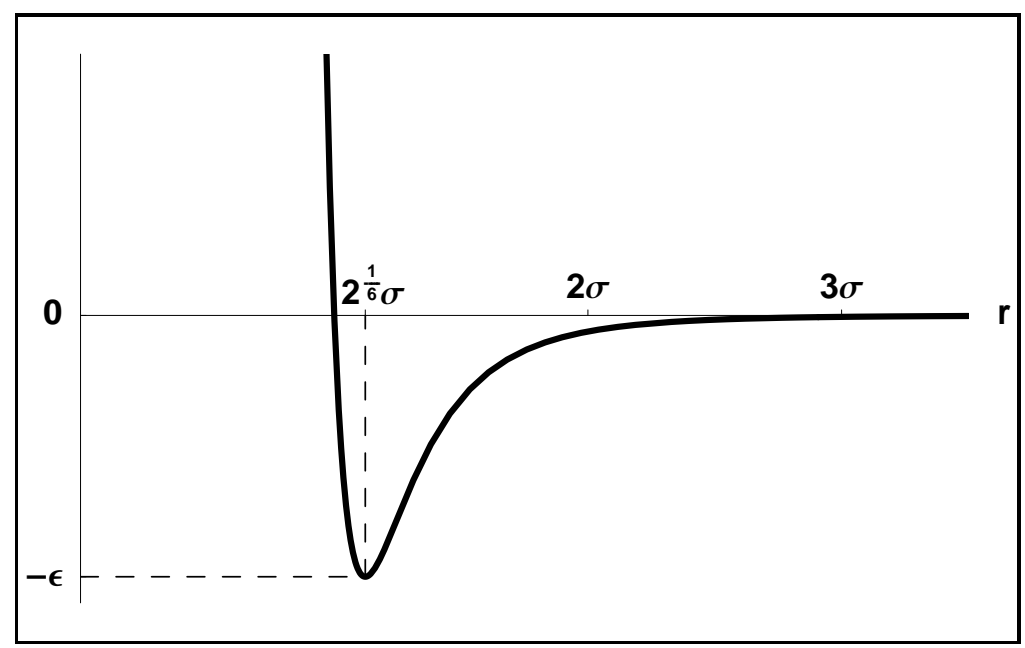

Figure 3.1: The Lennard-Jones Potential $U_{L J}(r)$

In addition to radial symmetry, we make two assumptions about the form of the pair potential $U(r)$ :

(1) $U(r) \geq C$ for some constant $C>-\infty$.

(2) As $r \rightarrow \infty, U(r) \rightarrow 0$ faster than $1 / r^{d}$.

Condition (1) ensures that the canonical density (2.24) can be normalized, whereas condition (2) will be needed to guarantee the convergence of certain integrals that appear in the next chapter. Note that (2) also precludes consideration of the Coulomb potential, which falls off as $1 / r^{d-2}$.

There are several pair potentials commonly used in MD that satisfy spherical symmetry, as well as conditions (1) and (2) above. These include, for example, the hard-sphere potential,

$$
U_{H S}(r)= \begin{cases}+\infty & r \leq \sigma \\ 0 & r>\sigma\end{cases}
$$

which describes the interaction of rigid spheres of diameter $\sigma$ undergoing elastic collisions. This was the first potential to be studied via an MD simulation on a computer [1].

A more realistic alternative to the hard-sphere potential in $d=3$ is the Lennard-Jones potential [35],

$$
U_{L J}(r)=4 \epsilon\left(\left(\frac{\sigma}{r}\right)^{12}-\left(\frac{\sigma}{r}\right)^{6}\right)
$$

which is shown in Figure 3.1. Here $\sigma$ is the scale length of the interaction, and $\epsilon$ is the depth of the potential well. The attractive $r^{-6}$ part of (3.3) comes from the van der Waal force, whereas the form of the repulsive $r^{-12}$ contribution has no physical basis, but was desirable in the early decades of MD for reasons of computational convenience: given $r^{-6}$, one could compute $r^{-12}$ rapidly by performing a single squaring operation. The LennardJones potential has been shown to yield good agreement with the properties of spherically symmetric molecules, such as $\mathrm{Ar}$ and $\mathrm{CH}_{3}$.

Several other potentials commonly used in MD, such as the Morse potential [45] or the Buckingham potential [5], are of a similar shape to $U_{L J}$, but conform more closely to real 
interparticle interactions. Nevertheless, the Lennard-Jones potential has been extensively studied via MD simulation over the years $[3,36,37,52,65,66]$, and a great deal is known about its behavior. It is usually the first potential used to test any new MD scheme. For these reasons, and for its simplicity, we shall use the Lennard-Jones potential in all numerics, although our analytical results will remain general.

\subsection{Optimal Prediction and the Molecular Dynamics Hamil- tonian}

The equations of motion generated by the Hamiltonian (3.1) are

$$
\begin{aligned}
\frac{d}{d t} q_{i} & =\frac{1}{m} p_{i} & q_{i}(0) & =x_{i} \\
\frac{d}{d t} p_{i} & =F_{i}(q) & p_{i}(0) & =y_{i},
\end{aligned}
$$

where

$$
F_{i}(q) \equiv-\nabla_{q_{i}} \phi(q)=-\sum_{j \neq i}^{N} \nabla_{q_{i}} U\left(\left|q_{i}-q_{j}\right|\right)
$$

is the force on the $i^{\text {th }}$ particle. Given these, we can make some simplifications to equations (2.20) and (2.21). The Liouvillian (2.22) becomes

$$
L=\sum_{i=1}^{N}\left\{\frac{1}{m} y_{i} \cdot \nabla_{x_{i}}-\left(\nabla_{x_{i}} \phi(x)\right) \cdot \nabla_{y_{i}}\right\} .
$$

We then find that

$$
\mathbb{P} L \widehat{x}_{i}=\mathbb{P} \frac{1}{m} \widehat{y}_{i}=\frac{1}{m} \widehat{y}_{i}
$$

and

$$
\mathbb{Q} L \widehat{x}_{i}=L \widehat{x}_{i}-\mathbb{P} L \widehat{x}_{i}=\frac{1}{m} \widehat{y}_{i}-\mathbb{P} \frac{1}{m} \widehat{y}_{i}=0,
$$

in which case $\mathscr{R}_{i}^{(q)}(\widehat{x}, \widehat{y})=\widehat{y}_{i} / m$ and $\mathscr{K}_{i}^{(q)}(\widehat{x}, \widehat{y}, t)=\mathscr{F}_{i}^{(q)}(x, y, t)=0$. Dropping the superscripts on $\mathscr{R}_{i}^{(p)}, \mathscr{K}_{i}^{(p)}$, and $\mathscr{F}_{i}^{(p)}$, equation (2.20) becomes

$$
\begin{aligned}
\frac{d}{d t} \widehat{q}_{i} & =\frac{1}{m} \widehat{p}_{i} \\
\frac{d}{d t} \widehat{p}_{i} & =e^{t L} \mathscr{R}_{i}(\widehat{x}, \widehat{y})+\int_{0}^{t} d s e^{(t-s) L} \mathscr{K}_{i}(\widehat{x}, \widehat{y}, s)+\mathscr{F}_{i}(x, y, t),
\end{aligned}
$$

where $\mathscr{R}_{i}(\widehat{x}, \widehat{y}), \mathscr{K}_{i}(\widehat{x}, \widehat{y}, s)$, and $\mathscr{F}_{i}(x, y, t)$ are as defined in $(2.21)$. This cannot be simplified further without making some approximations. The majority of this thesis will consist of the formulation and testing of various approximations to (3.7).

\subsection{Thermodynamic Quantities}

Equations (3.4) can be integrated numerically to yield $q_{i}(t)$ and $p_{i}(t)$ for all of the $N$ particles that make up the system. We will describe a method for accomplishing this in the next 
section. However, the details of the trajectories of individual particles are not our primary concern. We are more interested in macroscopic properties of the bulk fluid. There are two types of quantities that concern us. First, there are static thermodynamic averages, such as energy or pressure, which depend only on the instantaneous distribution of the positions and momenta. Second, there are autocorrelation functions of dynamical variables such as the velocity or shear stress, which may be used to recover transport coefficients via the appropriate Green-Kubo relation [17]. Dynamical correlation functions depend on the collective statistics of the particle trajectories, rather than on the details of particular molecular paths. These macroscopic properties of the system will be used to evaluate the quality of approximations that we will make to $(2.20)$.

We assume that the system is in thermodynamic equilibrium, and that the positions and momenta are distributed according to the canonical density at temperature $T$. Given any dynamical variable $A(q, p)$, we can use (2.24) to find its equilibrium average:

$$
\langle A(q, p)\rangle_{C}=\frac{\int d q \int d p A(q, p) e^{-\beta H(q, p)}}{\int d q \int d p e^{-\beta H(q, p)}},
$$

where $\langle\ldots\rangle_{C}$ denotes an average over the canonical density. Many of the interesting macroscopic properties of molecular systems can be written in terms of equilibrium averages.

The energy $E$, pressure $P$, and constant volume heat capacity $C_{V}$ are given by [3]:

$$
\begin{aligned}
E & =\langle K\rangle_{C}+\langle\phi\rangle_{C} \\
P & =\frac{2}{V d}\left(\langle K\rangle_{C}-\langle\mathcal{W}(q)\rangle_{C}\right) \\
C_{V} & =\frac{1}{k_{B} T^{2}}\left(\left\langle K^{2}\right\rangle_{C}-\langle K\rangle_{C}^{2}+\left\langle\phi^{2}\right\rangle_{C}-\langle\phi\rangle_{C}^{2}\right)
\end{aligned}
$$

where $K=\sum_{i=1}^{N} \frac{\left|p_{i}\right|^{2}}{2 m}$ is the kinetic part of the Hamiltonian (3.1), and

$$
\mathcal{W}(q)=\frac{1}{2} \sum_{i<j}^{N}\left|q_{i j}\right| U^{\prime}\left(\left|q_{i j}\right|\right)
$$

is called the virial. (Here we have defined $q_{i j}=q_{i}-q_{j}$.) Using (3.8), as well as the separability of $H$ into a kinetic part depending solely on $p$ and a potential part depending solely on $q$, it is simple to show that

$$
\begin{aligned}
\langle K\rangle_{C} & =\frac{d}{2} N k_{B} T \\
\left\langle K^{2}\right\rangle_{C} & =\left(k_{B} T\right)^{2}\left(\frac{d}{2} N+\frac{d^{2}}{4} N^{2}\right),
\end{aligned}
$$

in which case $E, P$, and $C_{V}$ become

$$
\begin{aligned}
E & =\frac{d}{2} N k_{B} T+\langle\phi\rangle_{C} \\
P & \left.=\rho k_{B} T-\frac{2}{V d}\langle\mathcal{W}(q)\rangle_{C} \quad \text { (Recall that } \rho=N / V\right) \\
C_{V} & =\frac{d}{2} N k_{B}+\frac{1}{k_{B} T^{2}}\left(\left\langle\phi^{2}\right\rangle_{C}-\langle\phi\rangle_{C}^{2}\right) .
\end{aligned}
$$


These are the expressions that we will be evaluating numerically.

We define the time average of a dynamical variable $A(q, p)$ as

$$
\langle A(q, p)\rangle_{t}=\lim _{\tau \rightarrow \infty} \frac{1}{\tau} \int_{0}^{\tau} d t A(q(t), p(t)) .
$$

In order to easily extract $\langle A(q, p)\rangle_{C}$ from the solution to (3.4) that will be generated numerically, we make the additional assumption that

$$
\langle A(q, p)\rangle_{t}=\langle A(q, p)\rangle_{C}
$$

This is known as the ergodic hypothesis, and is a common assumption in statistical physics, though no proof of it exists for any except the most simple systems.

In addition to the static properties $E, P$, and $C_{V}$, we will also be studying the velocity and shear stress temporal autocorrelation functions. These are commonly evaluated in MD simulations because they are related via Green-Kubo relations to the diffusion coefficient and shear viscosity, respectively. The diffusion coefficient is given by

$$
D=\int_{0}^{\infty} d t C_{v v}(t)
$$

where

$$
C_{v v}(t)=\frac{1}{3}\left\langle v_{i}(0) \cdot v_{i}(t)\right\rangle_{C}
$$

is the velocity autocorrelation function for an arbitrary particle $i$. The shear viscosity is

$$
\eta=\frac{V}{k_{B} T} \int_{0}^{\infty} d t C_{\Xi \Xi}(t)
$$

where

$$
C_{\Xi \Xi}(t)=\left\langle\Xi_{\mu \nu}(0) \Xi_{\mu \nu}(t)\right\rangle_{C} \quad(\mu \neq \nu, \text { no summation })
$$

is the autocorrelation function of $\Xi_{\mu \nu}$, an off-diagonal component of the stress tensor. For purposes of MD simulation, $\Xi_{\mu \nu}$ is best written in the form:

$$
\Xi_{\mu \nu}=\frac{1}{V} \sum_{i=1}^{N}\left(\frac{1}{m} p_{i, \mu} p_{i, \nu}-q_{i, \mu} \frac{\partial}{\partial q_{i, \nu}} \phi(q)\right) .
$$

Here we define a convention, to be used throughout this thesis, that Roman indices $(i, j, \ldots)$ will indicate particle number, while Greek indices $(\mu, \nu, \ldots)$ will denote Cartesian components. Thus, for example, $p_{i \mu}$ is the $\mu^{\text {th }}$ component of $p_{i}$, the momentum vector of the $i^{\text {th }}$ particle.

\subsection{Numerical Methods}

\subsubsection{The Velocity Verlet Algorithm}

In the traditional practice of MD, a system such as (3.4) is solved numerically via a discretetime finite difference scheme with time step $\Delta t$. The typical limiting factor on the speed of any discrete MD algorithm is the evaluation of the forces on each particle due to all the 
others. With $N$ particles and a pairwise interaction, there are in principle $N(N-1) / 2$ separate interparticle forces to calculate. By careful use of various bookkeeping tricks, evaluation of the forces can be reduced for moderate $N$ from an $O\left(N^{2}\right)$ process to an $O(N)$ process. Nevertheless, it remains the most time-consuming part of the simulation. Therefore, the goal is to use a scheme that has as few force evaluations per timestep as possible.

Energy drift is another factor to consider when selecting a finite-difference method for (3.4). Many schemes, such as the popular $4^{\text {th }}$ order Runge-Kutta algorithm for general ODEs, result in a slow upward drift of the energy when applied to Hamiltonian systems. An important set of methods that avoid this energy drift is the class of symplectic methods, which preserve phase space volume, a property shared by the equations of motion themselves. This means that the volume of a set of points in the $(q, p)$ phase space is invariant under the map $(q(t), p(t)) \rightarrow(q(t+\Delta t), p(t+\Delta t))$ which defines the finitedifference scheme. Since energy drift over large timescales typically implies non-conservation of phase space volume, symplectic methods keep the energy constant, to within smallscale fluctuations. Furthermore, while discrete time integrators usually fail to conserve the Hamiltonian, symplectic methods exactly conserve a closely related quantity known as the "shadow Hamiltonian" $\bar{H}$. The shadow Hamiltonian is a function of $\Delta t$, in terms of which it can be written as a Taylor series:

$$
\bar{H}(q, p, \Delta t)=H(q, p)+\sum_{n=1}^{\infty} \Delta t^{n} \bar{H}_{n}(q, p) .
$$

Though it will require some modification to incorporate a thermostat and, later, aspects of OP, we will base our numerics around a particularly simple, efficient, and popular symplectic scheme known as the velocity Verlet method [62]. Given the positions $q_{i}$, momenta $p_{i}$, and accelerations $a_{i}$ of the $N$ particles at time $t$, we compute these quantities at time $t+\Delta t$ via the following algorithm:

$$
\begin{aligned}
p_{i}\left(t+\frac{\Delta t}{2}\right) & =p_{i}(t)+\frac{\Delta t}{2} m a_{i}(t) \\
q_{i}(t+\Delta t) & =q_{i}(t)+\frac{\Delta t}{m} p_{i}\left(t+\frac{\Delta t}{2}\right) \\
a_{i}(t+\Delta t) & =\frac{1}{m} F_{i}\left(q_{i}(t+\Delta t)\right) \\
p_{i}(t+\Delta t) & =p_{i}\left(t+\frac{\Delta t}{2}\right)+\frac{\Delta t}{2} m a_{i}(t+\Delta t) .
\end{aligned}
$$

Here $F_{i}$ is as defined in equation (3.5). The velocity Verlet method is explicit, second order in $\Delta t$, and has several useful qualities. It is time-reversible, simple to implement, and requires only one force evaluation per timestep. Though it necessitates the storage of an additional set of quantities, the accelerations $a_{i}$, this is not an impediment. In a typical MD simulation, speed, not memory, is the limitation on system size.

For the velocity Verlet scheme and the Hamiltonian (3.1), the shadow Hamiltonian $\bar{H}$ 
is $[60]$ :

$$
\begin{aligned}
\bar{H}(q, p, \Delta t) & =\sum_{i=1}^{N} \frac{\left|p_{i}\right|^{2}}{2 m}+\phi(q) \\
& +\Delta t^{2}\left\{\frac{1}{12 m^{2}} \sum_{i, j=1}^{N}\left(p_{i} \cdot \nabla_{q_{i}}\right)\left(p_{j} \cdot \nabla_{q_{j}}\right) \phi(q)-\frac{1}{24 m} \sum_{i=1}^{N}\left|\nabla_{q_{i}} \phi(q)\right|^{2}\right\}+O\left(\Delta t^{4}\right) .
\end{aligned}
$$

We see that (3.15) conserves a quantity that differs from the actual Hamiltonian only to order $\Delta t^{2}$.

\subsubsection{Boundary Conditions and Initial Conditions}

In addition to an integration scheme for (3.4), we must also specify a choice of initial conditions for the positions and momenta, as well as a description of boundary conditions. Though our analytical development assumes that the particles are confined within a general bounded region $\Omega$ in $d$ dimensions, all of our simulations will take place in $d=3$, and make use of periodic boundary conditions in a cubic box of side $L=(N / \rho)^{\frac{1}{3}}$. In the limit of a large system with a short-ranged potential, this difference in boundary assumptions becomes a simple computational convenience that should have negligible effect on the bulk properties of the material.

Ideally, the initial conditions would be drawn randomly from the canonical distribution (2.24). For the momenta, this is simple; each component $y_{i \mu}$ is drawn independently from a Gaussian distribution with mean zero and variance $m k_{B} T$. Given the complexity of the potential $\phi$, however, canonical sampling of the positions is difficult. Our goal is to simulate a box of volume $L^{3}$, filled with $N$ particles at a uniform number density $\rho$. An obvious and simple choice for the initial particle positions would be to select each independently from a uniform distribution spanning the entire box. Unfortunately, this is untenable. Most of the potentials commonly used in MD diverge positively as two particles approach one another. Unless the number density is extremely low, at least two of the $N$ uniformly selected particles will be very close to one another with overwhelming probability. This leads to an unphysical blowup of the total energy of the system. In order to avoid this, we choose instead to place the particles in a face-centered cubic lattice. Because this configuration is not a minimum of the free energy for gases or liquids, an equilibration phase will be required in our numerics, during which the initially ordered configuration will "melt".

\subsubsection{Thermostat}

The state of our system is uniquely specified by $T$ and $\rho$. As the fluid's microscopic structure leaves the initial ordered state, kinetic energy will quickly be lost to potential energy, and the instantaneous temperature

$$
\widehat{T}(p) \equiv \frac{2}{N d k_{B}} \sum_{i=1}^{N} \frac{\left|p_{i}\right|^{2}}{2 m}
$$

will drop. In order to maintain the state of our simulated system, some mechanism must be added to the equations of motion (3.4) and to the numerical scheme (3.15) to regulate the temperature. If this takes the form of a well-modeled interaction between the $N$ particles 
and a heat bath at temperature $T$, it will have the additional benefit of ensuring equilibration to the canonical distribution. Such a mechanism is known as a thermostat.

There are several kinds of thermostats in common use. In general, these can be divided into two categories: momentum scaling and artificial friction methods. The former includes simple scaling, in which all the momenta are multiplied by a factor $(T / \widehat{T})^{1 / 2}$ at every time step, or perhaps periodically. This has the effect of eliminating temperature drift by forcing the instantaneous temperature (3.17) to equal the desired system temperature $T$. Also in wide use is the more drastic Andersen thermostat [4], which re-selects all momenta from a Maxwellian distribution at random intervals. While these methods yield a canonical distribution, and are thus suited to the calculation of static averages, they adversely effect the dynamics, which makes it difficult to extract accurate correlation functions.

The class of artificial friction thermostats includes, for example, the Gaussian isokinetic thermostat [17], which involves adding a term to the equations of motion:

$$
\begin{aligned}
\frac{d}{d t} q_{i} & =\frac{1}{m} p_{i} \\
\frac{d}{d t} p_{i} & =F_{i}(q)-\lambda p_{i},
\end{aligned}
$$

where

$$
\lambda \equiv \frac{\sum_{i=1}^{N} p_{i} \cdot F_{i}(q)}{\sum_{i=1}^{N}\left|p_{i}\right|^{2}}
$$

is a Lagrange multiplier. It can be shown that this form for the friction coefficient $\lambda$ forces the kinetic energy to be constant. This method has several drawbacks. The resulting equilibrium distribution is not canonical, and there are no fluctuations in temperature, which are to be expected in a real system. For this reason, we choose to implement instead the widely used Nosé-Hoover thermostat, which has been proven to yield a canonical equilibrium, allows temperature fluctuations, and does not adversely change the dynamics [47, 26].

In the Nosé-Hoover scheme, the equations of motion are altered as follows:

$$
\begin{aligned}
\frac{d}{d t} q_{i} & =\frac{1}{m} p_{i} \\
\frac{d}{d t} p_{i} & =F_{i}(q)-\zeta p_{i} \\
\frac{d}{d t} \zeta & =\frac{1}{Q}\left(\sum_{i=1}^{N} \frac{\left|p_{i}\right|^{2}}{m}-N d k_{B} T\right)=\frac{N d k_{B}}{Q}(\widehat{T}(p)-T) .
\end{aligned}
$$

Here the heat bath coupling is modeled as a friction term with a time-dependent coefficient $\zeta$. The parameter $Q$ in (3.18) is known as the heat bath mass, though its units are not those of mass, but rather of energy $\cdot$ time $^{2}$. The choice of $Q$ is crucial to the efficient functioning of the thermostat. If $Q$ is too small, the resulting distribution will not be canonical. If $Q$ is too large, fluctuations in temperature will not be sufficiently damped.

Following Nosé [47], we may estimate the best value of $Q$ via the following procedure: Define the temperature deviation as $\delta T=\widehat{T}-T$. Applying (3.17) and (3.18) allows us to 
derive a set of equations of motion for $\zeta$ and $\delta T$ :

$$
\begin{aligned}
\frac{d}{d t} \zeta & =\frac{N d k_{B}}{Q} \delta T \\
\frac{d}{d t} \delta T & =-2 T \zeta+2 \zeta \delta T+\frac{2}{N d k_{B} m} \sum_{i=1}^{N} p_{i} \cdot F_{i}(q)
\end{aligned}
$$

Near equilibrium, we expect both $\zeta$ and $\delta T$ to be small. Therefore, to first order in $\zeta$ and $\delta T$, we may ignore the second term on the right hand side of (3.19). Furthermore, the third term has mean zero at equilbrium, and so may be regarded as "noise". Thus, the above system becomes:

$$
\begin{aligned}
\frac{d}{d t} \zeta & =\frac{N d k_{B}}{Q} \delta T \\
\frac{d}{d t} \delta T & \approx-2 T \zeta+\text { noise }
\end{aligned}
$$

This has the form of a noisy oscillator with natural frequency $\omega$, which is given by

$$
\omega^{2}=\frac{2 T N d k_{B}}{Q} .
$$

In order for this to make sense physically, we require that the period of oscillation $2 \pi / \omega$ correspond to $\tau_{o}$, which is defined to be a typical microscopic timescale of the system. This allows us to solve for $Q$, to obtain

$$
Q=\frac{1}{2 \pi^{2}} \tau_{o}^{2} N d k_{B} T
$$

Nosé [47] has found that the dynamics of the system at equilibrium are highly insensitive to the exact value of $\tau_{o}$, and so we will simply set $\tau_{o}=\sqrt{m \sigma^{2} / \epsilon}$, where $\sigma$ and $\epsilon$ are, respectively, a length scale and an energy scale for the potential.

In order to integrate (3.18), some changes must be made to the velocity Verlet scheme (3.15) to incorporate the thermostat. A simple algorithm for accomplishing this is the following modified velocity Verlet method:

$$
\begin{aligned}
p_{i}\left(t+\frac{\Delta t}{2}\right) & =p_{i}(t)+\frac{\Delta t}{2}\left(m a_{i}(t)-\zeta(t) p_{i}(t)\right) \\
q_{i}(t+\Delta t) & =q_{i}(t)+\frac{\Delta t}{m} p_{i}\left(t+\frac{\Delta t}{2}\right) \\
\zeta(t+\Delta t) & =\zeta(t)+\Delta t \frac{N d k_{B}}{Q}\left\{\widehat{T}\left[p\left(t+\frac{\Delta t}{2}\right)\right]-T\right\} \\
a_{i}(t+\Delta t) & =\frac{1}{m} F_{i}\left(q_{i}(t+\Delta t)\right) \\
p_{i}(t+\Delta t) & =p_{i}\left(t+\frac{\Delta t}{2}\right)+\frac{\Delta t}{2}\left(m a_{i}(t+\Delta t)-\zeta(t+\Delta t) p_{i}(t+\Delta t)\right)
\end{aligned}
$$

where the implicit step (5) can be rendered explicit by solving for $p_{i}(t+\Delta t)$, to yield

$$
p_{i}(t+\Delta t)=\frac{p_{i}\left(t+\frac{\Delta t}{2}\right)+\frac{\Delta t}{2} m a_{i}(t+\Delta t)}{1+\frac{\Delta t}{2} \zeta(t+\Delta t)} .
$$

The modified velocity Verlet algorithm, like the original version, is second order and simple to implement. 


\subsubsection{Potential Truncation and Neighbor Lists}

As mentioned above, the most time-consuming part of most MD algorithms is the force evaluation (step (3) of (3.15) or step (4) of (3.22)), which is an $O\left(N^{2}\right)$ process. The force on particle $i$ due to particle $j$ is

$$
F\left(\left|q_{i j}\right|\right)=-\nabla_{q_{i}} U\left(\left|q_{i j}\right|\right)=-\frac{q_{i j}}{\left|q_{i j}\right|} U^{\prime}\left(\left|q_{i j}\right|\right) .
$$

In a numerical algorithm, the most efficient way to evaluate $F$ for a pair of particles is by defining a quantity

$$
\omega(r) \equiv-\frac{1}{r} U^{\prime}(r),
$$

in which case $F\left(q_{i j}\right)=q_{i j} \omega\left(\left|q_{i j}\right|\right)$. The advantage of this is that if the potential $U(r)$ can be written as a function of $r^{2}$ alone, then $\omega(r)$ can as well. (The Lennard-Jones potential (3.3) has this property.) Since, during any given timestep, we have the components of the vector $q_{i j}=q_{i}-q_{j}$, but not its magnitude, this allows us to save time by evaluating the potential using $\left|q_{i j}\right|^{2}$, without also requiring the calculation of $\left|q_{i j}\right|$.

We can also reduce the number of particle pairs for which the interparticle force must be calculated, by introducing a cutoff radius $r_{c}$, beyond which $U, F$, and $\omega$ are set equal to zero. This is normally accomplished by defining a shifted potential $U_{c}$ that is close to $U$, but has the property that both $U_{c}(r)$ and $U_{c}^{\prime}(r)$ go smoothly to zero at $r=r_{c}$. The form usually chosen for $U_{c}$ is $[3]$ :

$$
U_{c}(r)=\left\{\begin{array}{ll}
U(r)-U\left(r_{c}\right)+\left(r_{c}-r\right) U^{\prime}\left(r_{c}\right) & r<r_{c} \\
0 & r \geq r_{c}
\end{array} .\right.
$$

However, we will instead use the slightly more complicated

$$
U_{c}(r)=\left\{\begin{array}{ll}
U(r)-U\left(r_{c}\right)+\frac{r_{c}{ }^{2}-r^{2}}{2 r_{c}} U^{\prime}\left(r_{c}\right) & r<r_{c} \\
0 & r \geq r_{c}
\end{array},\right.
$$

which has several advantages over the standard version (3.23). If $U(r)$ depends only on $r^{2}$, then the $U_{c}(r)$ we have chosen preserves this property. Furthermore, for the Lennard-Jones potential, the truncated potential (3.24) deviates less from the true potential than $(3.23)^{2}$. The truncated Lennard-Jones potential with $r_{c}=2.5 \sigma$ (a typical value for $r_{c}$ ) is shown in Figure 3.2, along with the original potential for comparison.

It is important to note that when we truncate the potential in the manner described by either (3.23) or (3.24), the new potential $U_{c}(r)$ has a discontinuous second derivative at $r=r_{c}$. This means that the two numerical schemes (3.15) and (3.22) are no longer strictly second order, and, in fact, become first order methods. In addition, the derivation of the shadow Hamiltonian requires more smoothness than is present in either (3.23) or (3.24), so (3.16) is no longer strictly valid either. Nevertheless, the velocity Verlet method (3.15), along with a truncated potential, has been used for decades with excellent results.

By truncating the potential, we eliminate the need to evaluate the force function for the majority of the $N(N-1) / 2$ particle pairs. However, every pair of particles must still be

\footnotetext{
${ }^{2}$ For all $r$ and for all reasonable $r_{c}$, the quantity $\left|U_{c}(r)-U(r)\right|$ is smaller for the truncated potential defined by (3.24) than for that defined by (3.23). The same can be said for the deviations in the $F$ and $\omega$ functions derived from the two truncated potentials.
} 


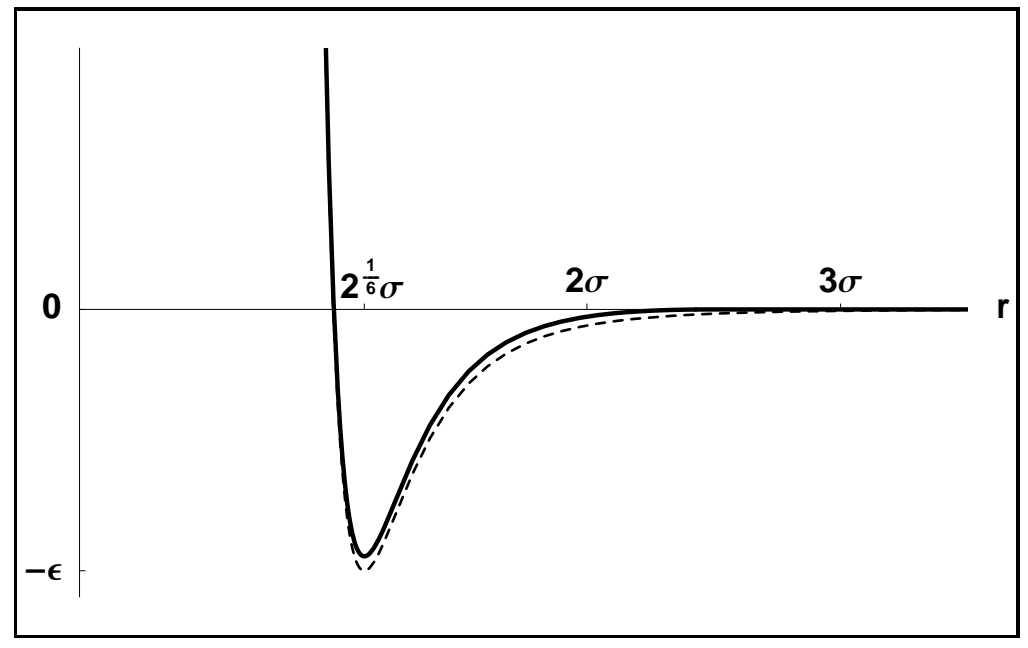

Figure 3.2: Solid line: The truncated Lennard-Jones Potential $U_{c}(r)$ with $r_{c}=2.5 \sigma$. Dashed line: The original Lennard-Jones potential.

checked to see if they fall within $r_{c}$ of each other. Thus, the force evaluation step remains an $O\left(N^{2}\right)$ process. The usual remedy for this is by use of a neighbor list [65, 64]. For each particle, we maintain a list of its most recent neighbors, where a "neighbor" is defined as any other particle less than a distance $r_{\ell}$ away, where $r_{\ell}>r_{c}$ is the neighbor list radius. For the Lennard-Jones potential, $r_{\ell}$ is normally chosen to be approximately $r_{c}+\sigma$.

Under the neighbor list method, we only check the distances between neighbors during a typical timestep. All other particle pairs are ignored. The interparticle force is then calculated for those neighbor pairs that fall within $r_{c}$ of each other. On average, the time needed for force evaluation will then be proportional to $\rho r_{c}{ }^{3} N$, rather than $N^{2}$. The neighbor list is updated periodically by inspecting all $N(N-1) / 2$ particle pair distances, and rebuilding the list according to which particles fall within $r_{\ell}$ of each other. This is an $O\left(N^{2}\right)$ process, but it is done at relatively widely-spaced intervals, so the overall process remains approximately $O(N)$, unless $N$ is quite large. Even in the case of large $N$, the neighbor list method significantly reduces the runtime.

\subsubsection{Timestep}

In any discrete integration scheme, the time step $\Delta t$ must be chosen carefully. Most shortrange interparticle potentials possess a scale length $\sigma$ that can serve both as a measure of the range of the force, as well as a rough estimate of the "size" of a molecule. In nature, this length is usually on the order of a few $\AA$. A good rule of thumb is that the timestep must be much shorter than the time it takes for a particle travelling at a typical thermal velocity to traverse one molecular length, i.e.

$$
\Delta t \ll \sigma \sqrt{\frac{m}{k_{B} T}} .
$$

In all our numerics, we therefore set

$$
\Delta t=\frac{\sigma}{100} \sqrt{\frac{m}{3 k_{B} T}},
$$


which is sufficiently small for most purposes.

\subsubsection{Lennard-Jones Reduced Units}

When studying the interaction of Lennard-Jones particles numerically, it is both traditional and useful to work in Lennard-Jones reduced units, in which all quantities are rendered dimensionless using appropriate combinations of the particle mass $m$, the scale length $\sigma$, the well depth $\epsilon$, and Boltzmann's constant $k_{B}$. The dimensionless quantities are then known as "reduced variables", and are denoted by a superscript asterisk: * . The relationships between several frequently used dimensional quantities and their reduced counterparts are shown below for reference.

$$
\begin{aligned}
& q^{*}=\frac{1}{\sigma} q \\
& p^{*}=\left(\frac{1}{m \epsilon}\right)^{\frac{1}{2}} p \\
& t^{*}=\left(\frac{\epsilon}{m \sigma^{2}}\right)^{\frac{1}{2}} t \\
& U^{*}\left(r^{*}\right)=\frac{1}{\epsilon} U\left(\sigma r^{*}\right) \\
& T^{*}=\frac{k_{B}}{\epsilon} T \\
& \rho^{*}=\sigma^{3} \rho \\
& C_{V}{ }^{*}=\frac{1}{k_{B}} C_{V} \\
& E^{*}=\frac{1}{\epsilon} E \\
& P^{*}=\frac{\sigma^{3}}{\epsilon} P
\end{aligned}
$$

The advantage of this is that all numerical results generated using reduced units are universal, and can be scaled to yield the corresponding quantity for any values of $m, \sigma$, and $\epsilon$. For this reason, almost all of the literature on the Lennard-Jones potential gives the results in reduced units.

\subsection{Phase Diagram of the Lennard-Jones System}

As a reference which will be used throughout this thesis, Figure 3.3 shows the phase diagram of the Lennard-Jones system in reduced units. The horizontal and vertical axes are density and temperature, respectively. Note that this system has a critical point at $\left(\rho_{c}^{*}, T_{c}^{*}\right)=(0.316,1.312)[50]$, above which there is no distinction between the gas and liquid phases. The various regions in the phase diagram carry labels that indicate the nature of the phase at that point:

G: Gas. Low-density subcritical fluid phase.

L: Liquid. High-density subcritical fluid phase.

S: Solid. In the solid state, the Lennard-Jones system crystalizes into either a face-centered cubic (FCC) or hexagonally close-packed (HCC) lattice, depending on the exact value of the density and temperature. Thus this system possesses solid-solid phase transitions between different packings, which are not indicated in Figure 3.3. These will not concern us in this thesis, however, as we will be focusing almost exclusively on the fluid phases.

S.C.: Supercritical Fluid. The single fluid state above the critical temperature. The lower bound of this state $\left(T^{*}=T_{c}^{*}\right)$ is indicated by the dotted line in Figure 3.3.

U: Unstable. A volume filled with a fluid that is initially at an unstable density and temperature will quickly and spontaneously separate into gas phase regions and liquid phase regions. Thus the unstable state points do not actually exist for a system at equilibrium. 


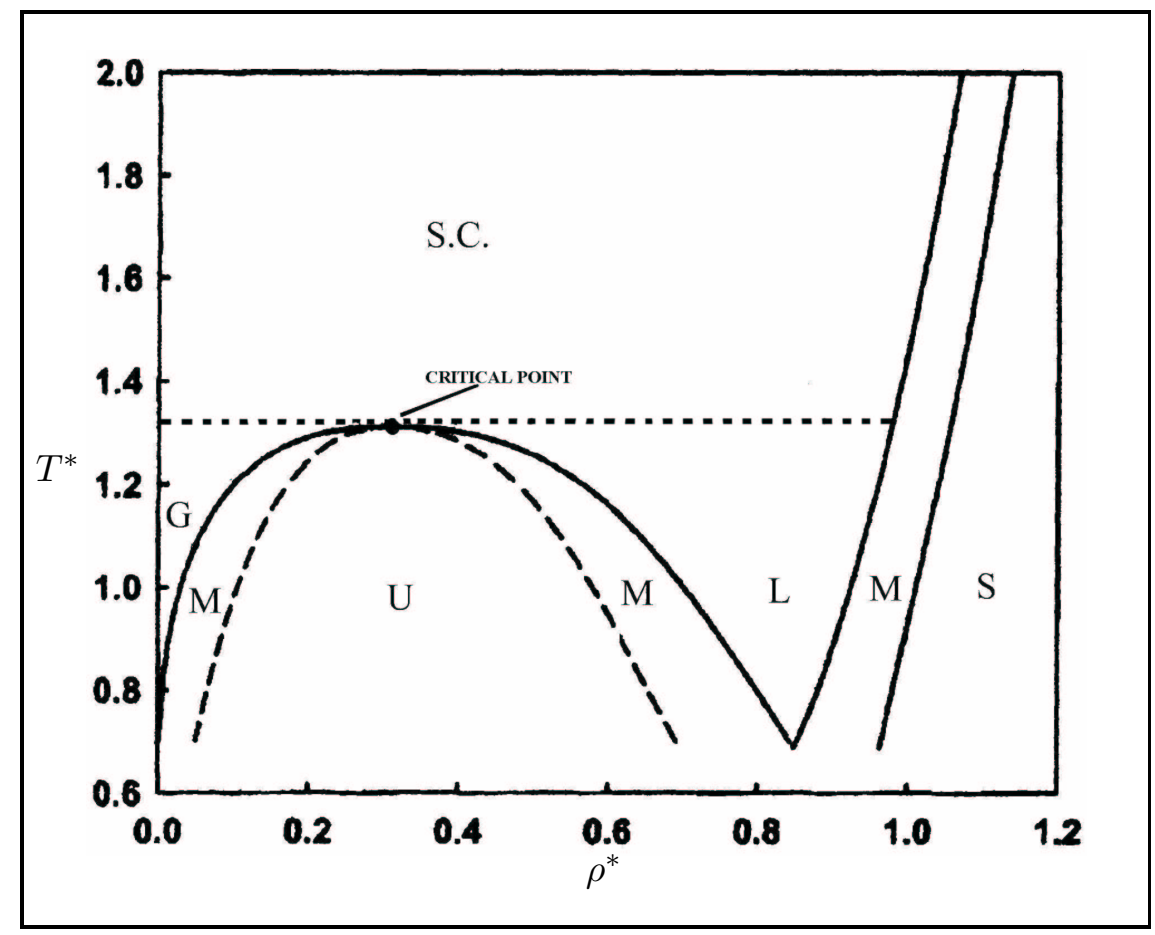

Figure 3.3: Phase diagram for the Lennard-Jones system in the $\left(\rho^{*}, T^{*}\right)$ plane. Solid lines: Phase boundaries. Dashed lines: Stability limits for liquid-gas equilibrium. Dotted line: Lower bound of supercritical fluid. (Image courtesy of Lin, Blanco, and Goddard [38])

M: Metastable. Metastable states, like unstable states, may spontaneously separate into regions of differing phase. However, they are neutrally stable to such separation. In addition to the gas-liquid metastable states, there is also a set of liquid-solid metastable states.

There are several methods by which the states in a numerical phase diagram such as Figure 3.3 are typically characterized during a MD simulation. Transitions to the solid phase may be identified, for example, by measuring the diffusion coefficient, which will be nearly zero for a solid, or by examining the Fourier transform of the particle density, which, for a crystaline state, will possess large contributions at the reciprocal vectors of the lattice [30]. Gases and liquids, on the other hand, can be distinguished by looking at the isothermal compressibility, which is much larger in a gaseous state.

Throughout this thesis, we will assume implicitly that the space in which our system of particles resides is isotropic. This will prove to be a useful assumption, both from the standpoint of conceptual simplicity and mathematical symmetry. The unstable and metastable states, because they are likely to contain boundaries between liquid and gaseous domains, are not spatially homogeneous, and so violate this assumption. For this reason, we will avoid subcritical temperatures in our numerical experiments by simulating only those state points that fall on the $T^{*}=1.6$ line in the $\left(\rho^{*}, T^{*}\right)$ plane. At the very least, this allows us to avoid the gas/liquid metastable/unstable region that appears below $T^{*}=T_{c}^{*}$. 


\section{Chapter 4}

\section{First Order Optimal Prediction}

We outline an approximation to the full equations of OP known as "First Order Optimal Prediction." This approximation implies the existence of a renormalized Hamiltonian governing the reduced system, for which we derive expressions in the low density limit, as well as in the general case. This approximation is tested by comparing several static and dynamical properties calculated from the reduced dynamics with those calculated from the full system.

\subsection{A Markovian Approximation to Optimal Prediction}

We will now construct a Markovian approximation to the general equation (2.17) for the evolution of the resolved variables. The components of the solution to the approximate equation will be denoted by $\bar{\varphi}_{i}$, where $1 \leq i \leq M$, in order to distinguish them from the components $\widehat{\varphi}_{i}$ of the exact solution to $(2.17)$.

We start by setting the memory and random forcing terms in (2.17) to zero, leaving only the first term, which yields

$$
\begin{aligned}
\frac{d}{d t} \widehat{\varphi}_{i}(t) & =e^{t L} \mathscr{R}_{i}(\widehat{z})=\mathscr{R}_{i}\left(e^{t L} \widehat{z}\right) \\
\widehat{\varphi}_{i}(0) & =\widehat{z}_{i} .
\end{aligned}
$$

We then approximate the propagator $e^{t L}$ associated with the full dynamics by $e^{t \mathscr{L}}$, where

$$
\mathscr{L} \equiv \sum_{i=1}^{M} \mathscr{R}_{i}(\widehat{z}) \partial_{\widehat{z}_{i}},
$$

which yields

$$
\begin{aligned}
\frac{d}{d t} \bar{\varphi}_{i}(\widehat{z}, t) & =\mathscr{R}_{i}(\bar{\varphi}(\widehat{z}, t)) \\
\bar{\varphi}_{i}(\widehat{z}, 0) & =\widehat{z}_{i} .
\end{aligned}
$$

An alternative, more intuitive, method of deriving (4.1) proceeds as follows. We start with the exact equation for the evolution of the resolved variables

$$
\begin{aligned}
\frac{d}{d t} \widehat{\varphi}_{i} & =\widehat{R}_{i}(\varphi) \\
\widehat{\varphi}_{i}(0) & =\widehat{z}_{i},
\end{aligned}
$$


where we have used the notation of Section 2.1. Note that evaluating the right hand side of (4.2) requires knowledge of $\widetilde{\varphi}$. We now assume that the distribution of the initial conditions is described by the probability density $f_{0}(z)$. This density evolves according to the Liouville equation (2.23), and at time $t$, the distribution of the $\varphi$ is $f(\varphi, t)$. By analogy with (2.9), and assuming that $\widehat{\varphi}(t)$ is known, we can use $f(\varphi, t)$ to define the time-dependent conditional expectation value of an arbitrary function $g(\varphi)$ as follows:

$$
E_{t}[g(\varphi) \mid \widehat{\varphi}(t)] \equiv \frac{\int d \widetilde{\varphi} g(\varphi) f(\varphi, t)}{\int d \widetilde{\varphi} f(\varphi, t)} .
$$

We now approximate the left hand side of (4.2) by its time-dependent conditional expectation value at time $t$, i.e.

$$
\begin{aligned}
\widehat{R}_{i}(\varphi(t)) & \approx E_{t}\left[\widehat{R}_{i}(\varphi) \mid \widehat{\varphi}(t)\right] \\
& =\frac{\int d \widetilde{\varphi} \widehat{R}_{i}(\varphi) f(\varphi, t)}{\int d \widetilde{\varphi} f(\varphi, t)} \\
& \equiv \mathscr{R}_{i}(\widehat{\varphi}, t) .
\end{aligned}
$$

If $f_{0}(z)$ is an invariant density, such as the canonical density, then $f(\varphi, t)=f_{0}(\varphi)$, and the form of $\mathscr{R}_{i}(\widehat{\varphi}, t)$ becomes time-independent. We then have $\mathscr{R}_{i}(\widehat{\varphi}, t)=e^{t L} E\left[\widehat{R}_{i}(z) \mid \widehat{z}\right]=$ $e^{t L} \mathbb{P} L \widehat{z}_{i}=\mathscr{R}_{i}(\widehat{\varphi})$, as before. Here $\mathbb{P}$ is the projection operator described in Chapter 2. This reproduces equation (4.1), and provides another reason why an invariant density on the initial condition $z$ is preferable.

Both of these methods involve uncontrolled approximations, and no attempt has been made at a rigorous justification. All that can be said is that according to the results of Section $2.2, \mathscr{R}_{i}(\widehat{z})=\mathbb{P} L \widehat{z}_{i}$ is the best approximation of $\widehat{R}_{i}(z)=L \widehat{z}_{i}$ by a function of $\widehat{z}$ alone. Thus, the flow of $\widehat{\varphi}$ generated by $\mathscr{R}$ is in some sense "close" to the flow generated by $\widehat{R}$.

The approximate system (4.1) has come to be known as First Order Optimal Prediction (FOOP), and is the form of OP found in the earliest papers on the topic [10, 11, 12]. These were written before the connection between (4.1) and the Mori-Zwanzig formalism was realized [7]. For Hamiltonian systems, FOOP takes the form

$$
\begin{aligned}
\frac{d}{d t} \bar{q}_{i} & =\mathscr{R}_{i}^{(q)}(\bar{q}, \bar{p}) \\
\frac{d}{d t} \bar{p}_{i} & =\mathscr{R}_{i}^{(p)}(\bar{q}, \bar{p}),
\end{aligned}
$$

where $\mathscr{R}_{i}^{(q)}(\widehat{x}, \widehat{y})=\mathbb{P} L \widehat{x}_{i}$ and $\mathscr{R}_{i}^{(p)}(\widehat{x}, \widehat{y})=\mathbb{P} L \widehat{y}_{i}$. We now introduce a useful theorem due to Hald (though a similar result was derived in a different context by Kirkwood in 1933 [29]):

Theorem 4.1 (Hald) For Hamiltonian systems governed by a Hamiltonian $H(x, y)$,

$$
\begin{aligned}
\mathscr{R}_{i}^{(q)}(\widehat{x}, \widehat{y}) & =\nabla_{\widehat{y}_{i}} \mathscr{H}(\widehat{x}, \widehat{y}) \\
\mathscr{R}_{i}^{(p)}(\widehat{x}, \widehat{y}) & =-\nabla_{\widehat{x}_{i}} \mathscr{H}(\widehat{x}, \widehat{y}),
\end{aligned}
$$

where

$$
\mathscr{H}(\widehat{x}, \widehat{y})=-k_{B} T \log \left(\frac{1}{c} \int d \widetilde{x} \int d \widetilde{y} e^{-\beta H(x, y)}\right) .
$$

(Here $c$ is an arbitrary constant with units of $\operatorname{action}^{(N-M) d}$.) 


\section{Proof:}

$$
\begin{aligned}
\mathscr{R}_{i}^{(q)}(\widehat{x}, \widehat{y}) & =\mathbb{P} L \widehat{x}_{i} \\
& =\mathbb{P}\left\{\nabla_{\widehat{y}_{i}} H(x, y)\right\} \\
& =\frac{\int d \widetilde{x} \int d \widetilde{y} \nabla_{\widehat{y}_{i}} H(x, y) e^{-\beta H(x, y)}}{\int d \widetilde{x} \int d \widetilde{y} e^{-\beta H(x, y)}} \\
& =\frac{-k_{B} T \nabla_{\widehat{y}_{i}} \int d \widetilde{x} \int d \widetilde{y} e^{-\beta H(x, y)}}{\int d \widetilde{x} \int d \widetilde{y} e^{-\beta H(x, y)}} \\
& =\nabla_{\widehat{y}_{i}}\left\{-k_{B} T \log \left(\frac{1}{c} \int d \widetilde{x} \int d \widetilde{y} e^{-\beta H(x, y)}\right)\right\}
\end{aligned}
$$

The expression for $\mathscr{R}_{i}^{(p)}(\widehat{x}, \widehat{y})$ follows analogously. In the last step above, we have inserted $c$ to keep the units consistent.

Hald's Theorem implies that (4.4) can be written as a Hamiltonian system:

$$
\begin{aligned}
\frac{d}{d t} \bar{q}_{i} & =\nabla_{\bar{p}_{i}} \mathscr{H}(\bar{q}, \bar{p}) & \bar{q}_{i}(0) & =\widehat{x}_{i} \\
\frac{d}{d t} \bar{p}_{i} & =-\nabla_{\bar{q}_{i}} \mathscr{H}(\bar{q}, \bar{p}) & \bar{p}_{i}(0) & =\widehat{y}_{i},
\end{aligned}
$$

where $\mathscr{H}$ is the renormalized Hamiltonian. This system can, in principle, be solved to yield trajectories for the $2 M \bar{q}_{i}$ and $\bar{p}_{i}$. However, the approximations we have made in order to arrive at (4.6), i.e. neglecting the memory and random forcing terms in (2.17), are drastic enough that we should not expect the $\bar{q}_{i}(t)$ and $\bar{p}_{i}(t)$ to accurately reproduce the correct dynamics of the $\widehat{q}_{i}$ and $\widehat{p}_{i}$. Nevertheless, the FOOP system will prove to be very useful. As we will show later in this chapter (see Theorem 4.2), numerical solutions to (4.6) can be used to exactly evaluate the canonical average of any function of the resolved coordinates alone. Furthermore, it can be proven that many common static properties of the full system, such as energy, pressure, and heat capacity, are expressible solely in terms of averages over functions of the resolved variables. Note, however, that there is no reason to conclude from this that FOOP can adequately reproduce temporal correlation functions or other quantities that depend strongly on the memory effects we are ignoring.

It is worth pausing at this point to note that if we had chosen to use either the linear projection $\mathbb{P}^{L}(2.12)$ or the finite rank projection $\mathbb{P}^{F R}(2.13)$ instead of $\mathbb{P}$, then Hald's Theorem would no longer hold. More importantly, when $M=N$, i.e. when all particles are resolved, we would fail to recover the original system of ODEs (3.4), which should be required for consistency in a real physical problem. Using $\mathbb{P}$, however, it is clear from the definition (4.5) that $\mathscr{H}=H$ when $M=N$, to within irrelevant constants. Qualitatively, this means that $\mathbb{P}$ is the only projection that preserves the essential "character" of the equations of motion.

\subsection{First Order Optimal Prediction for Molecular Dynamics}

We start with the MD system governed by the Hamiltonian (3.1). Our goal is to evaluate the associated renormalized Hamiltonian (4.5) by integrating out the coordinates $\widetilde{q}$ and $\widetilde{p}$ 
of the $N-M$ unresolved particles. As a shorthand, the resolved particles will be refered to as "r-particles", and the unresolved particles will be called "u-particles".

According to (4.5), the renormalized Hamiltonian is given by

$$
\begin{aligned}
e^{-\beta \mathscr{H}(\widehat{q}, \widehat{p})} & =\frac{1}{c} \int d \widetilde{q} \int d \widetilde{p} \exp \left[-\beta\left(\sum_{i=1}^{N} \frac{\left|p_{i}\right|^{2}}{2 m}+\phi(q)\right)\right] \\
& =\frac{1}{c} \exp \left[-\beta \sum_{i=1}^{M} \frac{\left|\widehat{p}_{i}\right|^{2}}{2 m}\right] \int d \widetilde{p} \exp \left[-\beta \sum_{i=1}^{N-M} \frac{\left|\widetilde{p}_{i}\right|^{2}}{2 m}\right] \int d \widetilde{q} e^{-\beta \phi(q)} .
\end{aligned}
$$

When working with the OP of MD, it is convenient to define the resolved number density $\widehat{\rho} \equiv M / V$ and the unresolved number density $\widetilde{\rho} \equiv(N-M) / V$. Note that $\widehat{\rho}+\widetilde{\rho}=N / V=\rho$. The thermodynamic limit now takes the form of assuming that $N, M, V \rightarrow \infty$, while $\hat{\rho}$ and $\widetilde{\rho}$ remain constant. As we will see below, if the unresolved density $\widetilde{\rho}$ is relatively low, (4.7) can be simplified further by expanding $\mathscr{H}$ as a Taylor series in $\widetilde{\rho}$. In general, however, the $\widetilde{q}$ integral is not analytically tractable, and we must resort to other methods. We will treat the low-density case first.

\subsubsection{Low-Density Diagrammatic Methods}

We begin by dividing the potential (3.2) into three parts:

$$
\phi(q)=\phi_{r r}(\widehat{q})+\phi_{r u}(\widehat{q}, \widetilde{q})+\phi_{u u}(\widetilde{q})
$$

where

$$
\begin{aligned}
\phi_{r r}(\widehat{q}) & \equiv \sum_{i<j}^{M} U\left(\left|\widehat{q}_{i}-\widehat{q}_{j}\right|\right), \\
\phi_{r u}(\widehat{q}, \widetilde{q}) & \equiv \sum_{i=1}^{M} \sum_{j=1}^{N-M} U\left(\left|\widehat{q}_{i}-\widetilde{q}_{j}\right|\right),
\end{aligned}
$$

and

$$
\phi_{u u}(\widetilde{q}) \equiv \sum_{i<j}^{N-M} U\left(\left|\widetilde{q}_{i}-\widetilde{q}_{j}\right|\right) .
$$

Here $\phi_{r r}$ is the potential energy resulting solely from interactions among resolved particles ( "r-r" interactions), $\phi_{r u}$ represents the contribution from interactions between resolved and unresolved particles ("r-u" interactions), and $\phi_{u u}$ is the potential energy resulting from interactions among unresolved particles ("u-u" interactions). If we now choose the arbitrary constant $c$ to be $(N-M) ! h^{(N-M) d}$, where $h$ is Planck's constant, then, after some minor rearrangement, (4.7) becomes

$$
\begin{aligned}
\mathscr{H}(\widehat{q}, \widehat{p})= & \sum_{i=1}^{M} \frac{\left|\widehat{p}_{i}\right|^{2}}{2 m}+\phi_{r r}(\widehat{q})-k_{B} T \log \left\{\frac{\int d \widetilde{q} e^{-\beta\left[\phi_{r u}(\widehat{q}, \widetilde{q})+\phi_{u u}(\widetilde{q})\right]}}{\left.\int d \widetilde{q} e^{-\beta \phi_{u u}(\widetilde{q})}\right\}}\right. \\
& -k_{B} T \log \left\{\frac{1}{(N-M) ! h^{(N-M) d}} \int d \widetilde{p} \int d \widetilde{q} \exp \left[-\beta\left(\sum_{i=1}^{N-M} \frac{\left|\widetilde{p}_{i}\right|^{2}}{2 m}+\phi_{u u}(\widetilde{q})\right)\right]\right\} .
\end{aligned}
$$


The third quantity on the right hand of (4.11) is a constant that is equal to the Helmholtz free energy $F(N-M, \widetilde{\rho}, T)$ of $N-M$ interacting particles at density $\widetilde{\rho}$ and temperature $T$ [48]. Furthermore, we define

$$
H_{G}(\widehat{q}, \widehat{p}) \equiv \sum_{i=1}^{M} \frac{\left|\widehat{p}_{i}\right|^{2}}{2 m}+\phi_{r r}(\widehat{q}) .
$$

$H_{G}$ is the Hamiltonian of the system

$$
\begin{aligned}
\frac{d}{d t} \widehat{q}_{i} & =\frac{1}{m} \widehat{p}_{i} \\
\frac{d}{d t} \widehat{p}_{i} & =-\sum_{j \neq i}^{M} \nabla_{\widehat{q}_{i}} U\left(\left|\widehat{q}_{i}-\widehat{q}_{j}\right|\right),
\end{aligned}
$$

which is obtained from the full system (3.4) by neglecting all of the unresolved degrees of freedom $\widetilde{q}$ and $\widetilde{p}$. The subscript " $G$ " alludes to the fact that this is reminiscent of the Galerkin method of system reduction for ODEs and boundary value problems [28], and we will refer to (4.13) as the "Galerkin system".

The Galerkin system will frequently be used as a "zeroth order" approximation to the exact dynamics (3.7) of the reduced system. Since (4.13) describes a fluid in the state $(\widehat{\rho}, T)$, we would not expect its macroscopic properties to accurately mirror those of the full system, which is in the state $(\rho, T)$. However, we will use the Galerkin system as an example of a bad approximation to (3.7), which will serve as a comparison to FOOP, as well as other, hopefully better, approximations to be made in Chapter 5 .

The renormalized Hamiltonian now becomes

$$
\mathscr{H}(\widehat{q}, \widehat{p})=F(N-M, \widetilde{\rho}, T)+H_{G}(\widehat{q}, \widehat{p})-k_{B} T \log \left\{\frac{\int d \widetilde{q} e^{-\beta\left[\phi_{r u}(\widehat{q}, \widetilde{q})+\phi_{u u}(\widetilde{q})\right]}}{\int d \widetilde{q} e^{-\beta \phi_{u u}(\widetilde{q})}}\right\} .
$$

The first two terms of (4.14) represent the contributions to $\mathscr{H}$ of the unresolved and resolved subsystems taken in isolation, whereas the third term describes the correction due to their interaction.

The correction term can be divided into two parts as follows:

$$
\begin{aligned}
& \log \left\{\frac{\int d \widetilde{q} e^{-\beta\left[\phi_{r u}(\widehat{q}, \widetilde{q})+\phi_{u u}(\widetilde{q})\right]}}{\left.\int d \widetilde{q} e^{-\beta \phi_{u u}(\widetilde{q})}\right\}=}\right. \\
& \quad \log \left\{\frac{1}{V^{N-M}} \int d \widetilde{q} e^{-\beta\left[\phi_{r u}(\widehat{q}, \widetilde{q})+\phi_{u u}(\widetilde{q})\right]}\right\}-\log \left\{\frac{1}{V^{N-M}} \int d \widetilde{q} e^{-\beta \phi_{u u}(\widetilde{q})}\right\} .
\end{aligned}
$$

Each of these two quantities can be expanded for small $\widetilde{\rho}$ in a diagrammatic series. The argument of the second logarithm is simpler, and we will tackle it first. Following the methods of Mayer and Mayer [41] and Mayer and Montroll [42], we define a function

$$
f(r) \equiv e^{-\beta U(r)}-1 .
$$


In terms of $f$, we can write

$$
\begin{aligned}
e^{-\beta \phi_{u u}(\widetilde{q})} & =\prod_{i<j}^{N-M} e^{-\beta U\left(\left|\widetilde{q}_{i}-\widetilde{q}_{j}\right|\right)} \\
& =\prod_{i<j}^{N-M}\left(f_{i j}+1\right) \quad\left(f_{i j} \equiv f\left(\left|\widetilde{q}_{i}-\widetilde{q}_{j}\right|\right)\right) \\
& =1+\sum_{\{i j\}} f_{i j}+\sum_{\{i j\}} \sum_{\{k l\}} f_{i j} f_{k l}+\sum_{\{i j\}} \sum_{\{k l\}} \sum_{\{m n\}} f_{i j} f_{k l} f_{m n}+\cdots,
\end{aligned}
$$

where $\sum_{\{i j\}}$ indicates a sum over all distinct pairs of u-particles, $\sum_{\{i j\}} \sum_{\{k l\}}$ indicates a sum over all distinct pairs of pairs, and so on. The Mayer $f$-function is convenient to work with because it is short ranged: $f(r) \rightarrow-\beta U(r)$ for large $r$, so $f$ goes to zero asymptotically at the same rate as $U$. This is in contrast to the behavior of $\exp (-\beta U(r))$, which goes to 1 as $r \rightarrow \infty$.

The argument of the second logarithm in the correction term (4.15) can now be written as

$$
\begin{aligned}
& \frac{1}{V^{N-M}} \int d \widetilde{q} e^{-\beta \phi_{u u}(\widetilde{q})}= \\
& \quad \frac{1}{V^{N-M}} \int d \widetilde{q}\left\{1+\sum_{\{i j\}} f_{i j}+\sum_{\{i j\}} \sum_{\{k l\}} f_{i j} f_{k l}+\sum_{\{i j\}} \sum_{\{k l\}} \sum_{\{m n\}} f_{i j} f_{k l} f_{m n}+\cdots\right\} .
\end{aligned}
$$

The first quantity in brackets above yields a 1 , since $\int d \widetilde{q}=V^{N-M}$. The remaining terms in (4.16) are integrals over all $N-M$ of the $\widetilde{q}_{i}$, where the integrands consist of one or more $f$-functions, the arguments of which involve some subset of the $\widetilde{q}_{i}$. These integrals are best depicted diagrammatically using labeled graphs known as Mayer diagrams. We represent the $i^{\text {th }}$ u-particle by a labeled, filled node: $\bullet^{i}$. An " $f$-bond" linking particles $i$ and $j$ contributes a factor $f_{i j}$ to the integrand, and is represented by a line joining the nodes labeled $i$ and $j:{ }^{i} \longrightarrow{ }^{j}$. The way in which the various integrals in (4.16) are represented by diagrams is best illustrated by example:

$$
\begin{aligned}
& \overbrace{7}^{2}=\frac{1}{V} \int d \widetilde{q}_{1} \int d \widetilde{q}_{2} f_{1,2} \\
& =\frac{1}{V} \int d \widetilde{q}_{3} \int d \widetilde{q}_{4} \int d \widetilde{q}_{7} f_{3,4} f_{4,7} \\
& \underbrace{1}_{2}=\frac{1}{V} \int d \widetilde{q}_{4} \int d \widetilde{q}_{9} \int d \widetilde{q}_{1} \int d \widetilde{q}_{2} f_{4,9} f_{9,1} f_{9,2} f_{1,2} \quad \text { etc... }
\end{aligned}
$$

The purpose of the $1 / V$ factor is to ensure that the diagrams are intensive quantities. Each $\widetilde{q}_{i}$ that takes part in a diagram is integrated over the whole domain $\Omega$. In the first example above, we can fix $\widetilde{q}_{1}$ as the origin of the $\widetilde{q}_{2}$ integration. Unless $\widetilde{q}_{1}$ happens to be within a few multiples of the potential scale length $\sigma$ of $\partial \Omega$, where $\partial \Omega$ is the boundary of 
$\Omega$, we then have:

$$
\begin{aligned}
\bullet & =\frac{1}{V} \int d \widetilde{q}_{1} \int d \widetilde{q}_{2} f_{1,2} \\
& =\frac{1}{V} \int d \widetilde{q}_{1} \int d \widetilde{q}_{2} f\left(\left|\widetilde{q}_{2}\right|\right) \\
& =\int d \widetilde{q}_{2} f\left(\left|\widetilde{q}_{2}\right|\right),
\end{aligned}
$$

A similar argument shows that the $1 / V$ is necessary to cancel an extraneous volume factor for each diagram. The boundary effects alluded to above are restricted to a thin "skin" of volume $\sim \sigma|\partial \Omega|$. Since $\sigma$ is of the order of several $\AA$, this constitutes a negligible fraction of the total volume, and we will ignore this effect. Note that such boundary effects may not be ignored when considering a long-range interaction such as the Coulomb potential.

All of the diagrams shown above are examples of connected diagrams. A connected diagram has the property that it is possible to find a path joining any two of its nodes, where a "path" is defined as a sequence of nodes connected by bonds. The vast majority of the diagrams in the expansion (4.16) are unconnected diagrams, i.e. diagrams that can be divided into two or more smaller diagrams (known as "components") that are not connected by any bonds. The value of an unconnected diagram is the product of the values of the components that comprise it. Some examples of unconnected diagrams, along with their corresponding values, are shown below:

$$
\begin{aligned}
\bullet_{2}^{1} \bullet_{4}^{3} & =\left(\frac{1}{V} \int d \widetilde{q}_{1} \int d \widetilde{q}_{2} f_{1,2}\right)^{2} \\
\overbrace{6}^{5} & =\left(\frac{1}{V} \int d \widetilde{q}_{1} \int d \widetilde{q}_{2} \int d \widetilde{q}_{3} f_{1,2} f_{2,3}\right)\left(\frac{1}{V} \int d \widetilde{q}_{1} \int d \widetilde{q}_{2} \int d \widetilde{q}_{3} f_{1,2} f_{2,3} f_{1,3}\right)
\end{aligned}
$$

Here we have made use of the fact that the node labels are dummy indices that refer to variables of integration, and thus may be permuted or renamed without changing the value of the corresponding integral. For this reason, we will usually omit the labels altogether.

The connection set of a labeled diagram is the set of node index pairs that are connected by bonds. For example, the connection set for

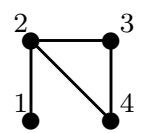

is $\{(1,2),(2,3),(2,4),(3,4)\}$. Two unlabeled diagrams are called topologically equivalent if they have the same number of nodes and bonds, and labeled versions of each can be found that have the same connection set. Two diagrams that are not topologically equivalent are called topologically distinct. Thus

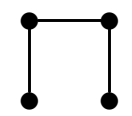

and

are topologically equivalent, whereas

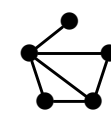

and

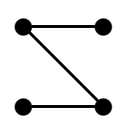

.

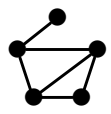


are topologically distinct.

Equation (4.16) is equivalent to the following sum of diagrams:

$$
\begin{aligned}
& \frac{1}{V^{N-M}} \int d \widetilde{q} e^{-\beta \phi_{u u}(\widetilde{q})} \\
& =1+\sum_{m=2}^{N-M}\left\{\begin{array}{c}
\text { A weighted sum of all topologically } \\
\text { distinct, unlabeled diagrams that have } \\
\text { exactly } m \text { nodes }
\end{array}\right\}
\end{aligned}
$$

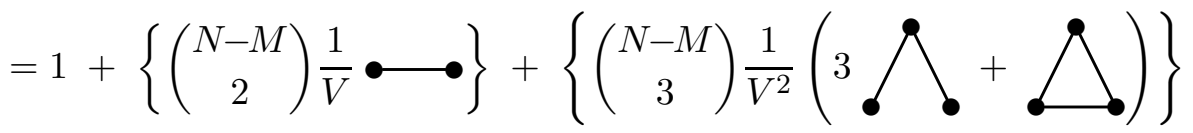

$$
\begin{aligned}
& +\left\{\frac{1}{2}\left(\begin{array}{l}
4 \\
2
\end{array}\right)\left(\begin{array}{c}
N-M \\
4
\end{array}\right) \frac{1}{V^{2}} \nmid\right.
\end{aligned}
$$

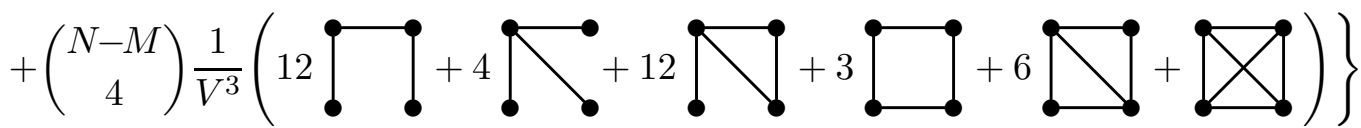

$$
\begin{aligned}
& +\cdots
\end{aligned}
$$

The coefficients in front of each diagram above can be determined from the following counting argument. Consider a diagram consisting of $m$ nodes and $k$ components, where $m \geq 2$ and $k \geq 1$. We define $n=m-k$ to be the number of "extra" nodes in the diagram (note that this nomenclature is nonstandard). The integral term in (4.16) that gives rise to this diagram initially has the prefactor $1 / V^{N-M}$. However, $N-M-m$ of these volume factors are immediately cancelled when we integrate out the coordinates of the u-particles that do not take part in the diagram. We must then remove another factor of $1 / V$ for each of the $k$ components, due to the $1 / V$ in the definitions of the diagrams. This leaves us with an overall prefactor of $1 / V^{m-k}=1 / V^{n}$. The remainder of the coefficient preceding each diagram above is a combinatorical factor representing the number of distinct ways of forming labeled versions of that diagram using the $N-M$ possible indices. This suggests that we may write the above sum as

$$
\begin{aligned}
& \frac{1}{V^{N-M}} \int d \widetilde{q} e^{-\beta \phi_{u u}(\widetilde{q})} \\
& =1+\sum_{n=1}^{N-M-1}\left(\frac{1}{V}\right)^{n}\left\{\begin{array}{c}
\text { A sum of all topologically distinct, unlabeled diagrams } \\
\text { with } n \text { extra nodes, weighted by the number of distinct } \\
\text { ways of forming each diagram from } N-M \text { u particles }
\end{array}\right\} \\
& =1+\frac{1}{V}\left\{\left(\begin{array}{c}
N-M \\
2
\end{array}\right) \bullet \longrightarrow\right\}
\end{aligned}
$$

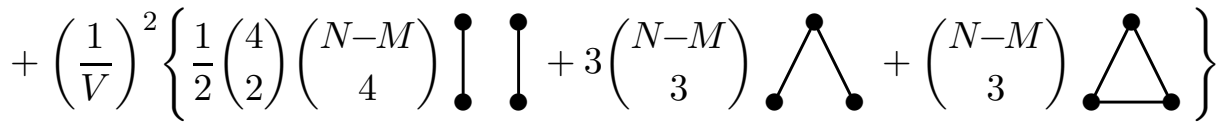

$$
\begin{aligned}
& +\cdots
\end{aligned}
$$


There is an explicit expression for the combinatorical factors that serve as weights in (4.17). Consider a general term involving a (possibly unconnected) diagram with $n$ extra nodes and $k$ components. If the $i^{\text {th }}$ component has $\alpha_{i}$ extra nodes, where $\sum_{i=1}^{k} \alpha_{i}=n$, then its total number of nodes will be $\alpha_{i}+1$. Furthermore, there are $N-M-n-k \mathrm{u}$-particles that are not associated with any node in the diagram. This implies that the number of distinct ways of constructing this diagram is

$$
\frac{1}{\mu(\alpha)}\left(\prod_{i=1}^{k} \gamma_{i}\right)\left(\begin{array}{c}
N-M \\
\alpha_{1}+1, \ldots, \alpha_{k}+1
\end{array}\right)
$$

where $\alpha=\left(\alpha_{1}, \ldots, \alpha_{k}\right)$ and

$$
\left(\begin{array}{c}
N-M \\
\alpha_{1}+1, \ldots, \alpha_{k}+1
\end{array}\right)=\frac{(N-M) !}{(N-M-n-k) ! \prod_{i=1}^{k}\left(\alpha_{i}+1\right) !}
$$

is the multinomial coefficient giving the number of ways of dividing $N-M$ elements into $k+1$ distinct sets of sizes $N-M-n-k, \alpha_{1}+1, \ldots, \alpha_{k}+1$. The $\gamma_{i}$ are geometrical factors, independent of $N$ and $M$, which describe the number of ways of forming the $i^{\text {th }}$ component, given $\alpha_{i}+1$ distinguishable nodes. There is no general formula that determines the $\gamma_{i}$, but they can usually be found easily by inspection. For example, the diagram

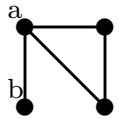

has $\gamma=12$, because for each of the four ways of assigning an index to node a, there are three ways of assigning one of the remaining indices to node $b$ (the final two nodes are equivalent). Finally, $\mu(\alpha)$ is a multiplicity term that has one factor of $p$ ! for every $\alpha_{i}$ whose value is repeated $p$ times. For example, $\mu((1,1,2,3,3,4,4,4,5))=2 ! 2 ! 3 !=24$. If all the $\alpha_{i}$ are different, then $\mu(\alpha)=1$. The purpose of $\mu$ is to account for the $p$ ! possible permutations of the indices among $p$ identical components. These permutations do not generate distinct diagrams, and would otherwise be multiply counted, i.e.

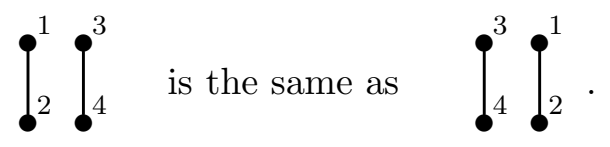

The condition $\sum_{i=1}^{k} \alpha_{i}=n$ implies that $\alpha \in P\{n\}$, where $P\{n\}$ is the set of partitions of $n$, i.e. $P\{1\}=\{(1)\}, P\{2\}=\{(1,1),(2)\}, P\{3\}=\{(1,1,1),(1,2),(3)\}$, etc. Many of the terms in (4.17) with identical $n$ also have the same values of $\alpha$ and $\mu(\alpha)$, as well as the same multinomial coefficient. We can therefore simplify (4.17) further by the following additional grouping of diagrams:

$$
\frac{1}{V^{N-M}} \int d \widetilde{q} e^{-\beta \phi_{\text {uu }}(\widetilde{q})}=1+\sum_{n=1}^{N-M-1}\left(\frac{1}{V}\right)^{n} \sum_{\alpha \in P\{n\}} \frac{1}{\mu(\alpha)}\left(\begin{array}{c}
N-M \\
\alpha_{1}+1, \ldots, \alpha_{k}+1
\end{array}\right) \prod_{i=1}^{k} C_{\alpha_{i}}
$$

where

$$
C_{s}=\left\{\begin{array}{c}
\text { A sum of all topologically distinct, connected, unlabeled } \\
\text { diagrams with } s+1 \text { nodes, with each diagram preceeded } \\
\text { by its corresponding } \gamma \text { factor }
\end{array}\right\} .
$$


The diagrams described in the definition of $C_{s}$ are known as clusters in the physics literature, and so we will call $C_{s}$ the cluster sum ${ }^{1}$ of order $s$. The first few $C_{s}$ are listed below:

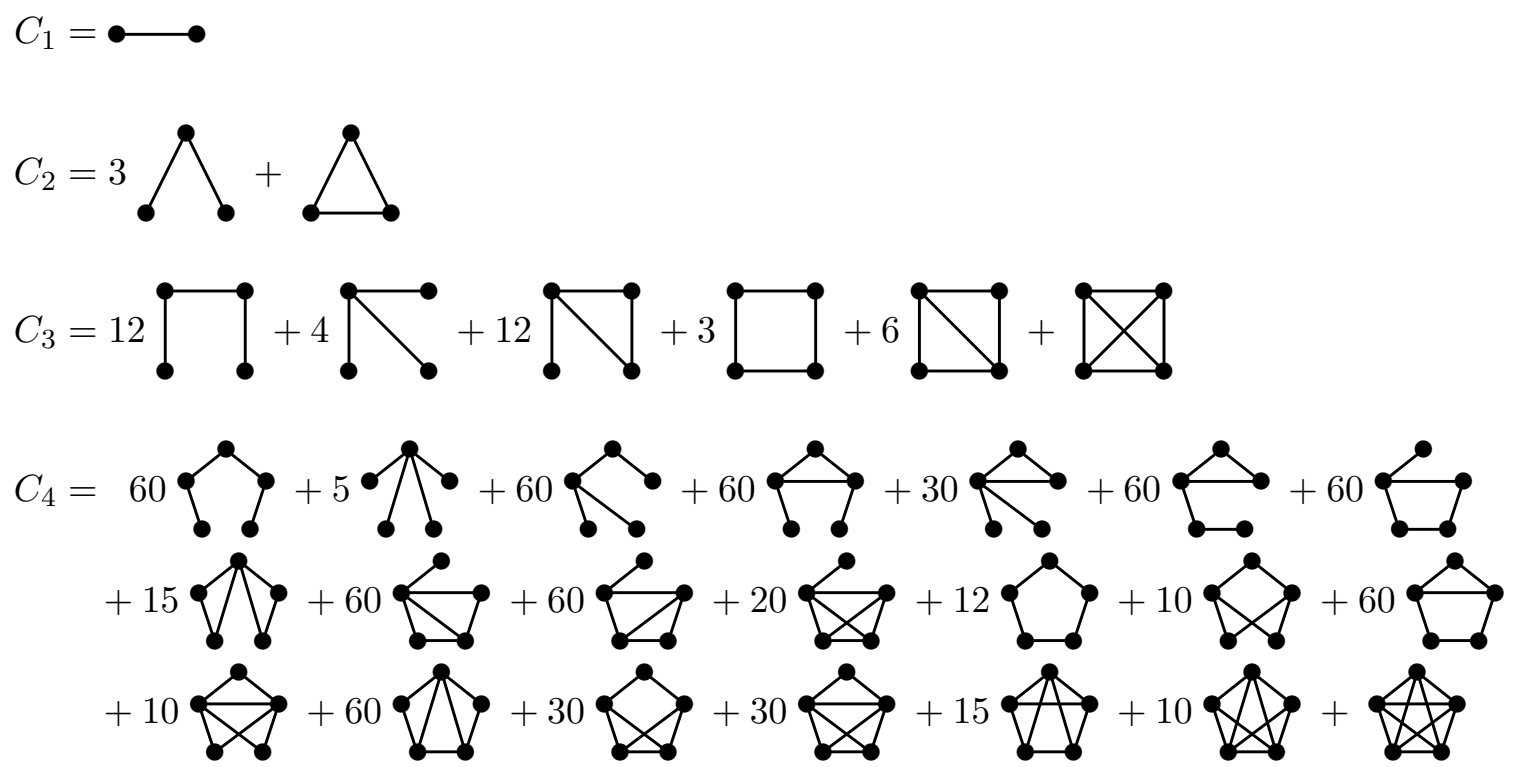

Note that the cluster sums, as well as the diagrams of which they are comprised, depend on $T$, but not on $N, M$, or $V$.

According to (4.15), we are interested in the logarithm of (4.18). Taylor expanding the logarithm in powers of $1 / V$ yields

$$
\begin{aligned}
\log \left\{\frac{1}{V^{N-M}} \int d \widetilde{q} e^{-\beta \phi_{\text {uu }}(\widetilde{q})}\right\} & =\log \left\{1+\sum_{n=1}^{N-M-1}\left(\frac{1}{V}\right)^{n} \theta_{n}\right\} \\
& =\sum_{n=1}^{\infty}\left(\frac{1}{V}\right)^{n} \psi_{n},
\end{aligned}
$$

where

$$
\theta_{n}=\sum_{\alpha \in P\{n\}} \frac{1}{\mu(\alpha)}\left(\begin{array}{c}
N-M \\
\alpha_{1}+1, \ldots, \alpha_{k}+1
\end{array}\right) \prod_{i=1}^{k} C_{\alpha_{i}},
$$

and the $\psi_{n}$ are to be determined. In principle, we can find the $\psi_{n}$ by a direct comparison of the expansions on either side of (4.19), but first we will need to introduce some more terminology from graph theory.

An articulation point of a connected diagram is a node whose removal causes the diagram to split into two or more disconnected components. A diagram that has an articulation point is called reducible, and can be written as a product of lower order diagrams by fixing the variable associated with the articulation node as the origin for the integrations over all other

\footnotetext{
${ }^{1}$ Mayer and Mayer instead used the related quantity $b_{s}=(1 / s !) C_{s}$, which they called the cluster integral of order $s$.
} 
node variables. For example,

$$
\begin{aligned}
\boldsymbol{\gamma} & =\frac{1}{V} \int d \widetilde{q}_{1} \int d \widetilde{q}_{2} \int d \widetilde{q}_{3} f_{1,2} f_{2,3} \\
& =\frac{1}{V} \int d \widetilde{q}_{2} \int d \widetilde{q}_{1} f\left(\left|\widetilde{q}_{1}\right|\right) \int d \widetilde{q}_{3} f\left(\left|\widetilde{q}_{3}\right|\right) \\
& =\left(\int d \widetilde{q}_{1} f\left(\left|\widetilde{q}_{1}\right|\right)\right)^{2}=(\bullet)^{2} .
\end{aligned}
$$

Diagrams without articulation points are called irreducible. We define $\bar{C}_{s}$ to be the irreducible part of $C_{s}$, i.e. it is equal to the order $s$ cluster sum with all reducible diagrams dropped. The first few $\bar{C}_{s}$ are

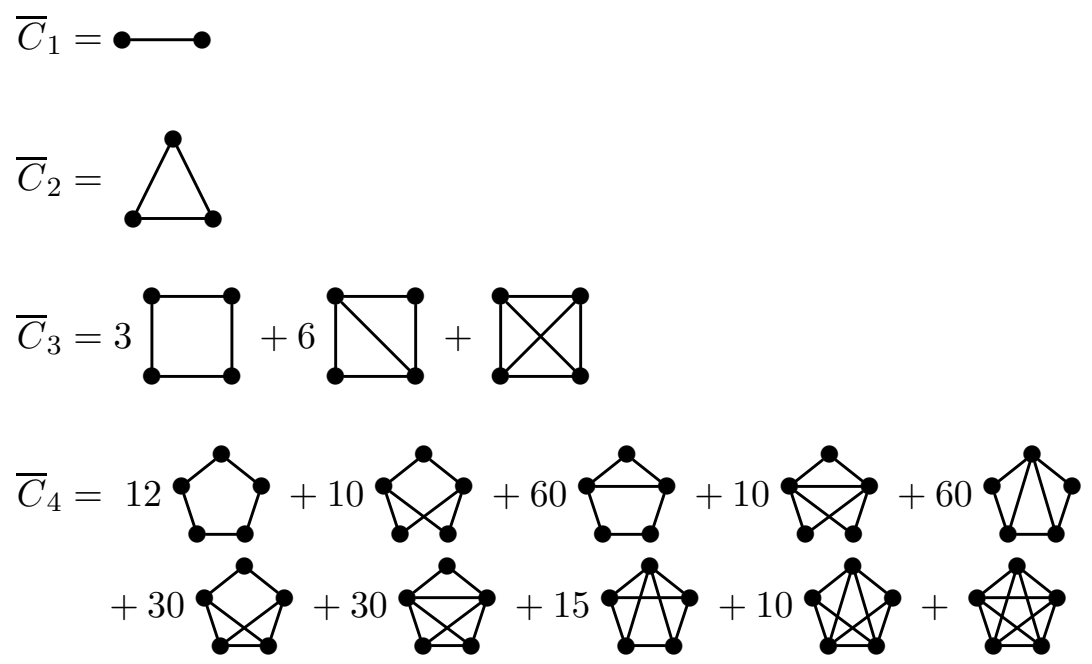

Returning to (4.19) and using the definition (4.20) of the $\theta_{n}$, we can obtain lengthy expressions for the first few $\psi_{n}$ in terms of the cluster sums $C_{s}$ (which will not be reproduced here). After inserting the expressions for the $C_{s}$ and passing to the thermodynamic limit, we find that a fortuitous cancellation occurs, and that the first few $\psi_{n}$ satisfy

$$
\psi_{n}=\frac{(N-M)^{n+1}}{(n+1) !} \bar{C}_{n}
$$

In fact, the above expression is generally true for all $n$. The proof of this is beyond the scope of this thesis, and can be found in [41]. Combining (4.21) and (4.19), and using the fact that $(N-M) / V=\widetilde{\rho}$, we finally arrive at

$$
\log \left\{\frac{1}{V^{N-M}} \int d \widetilde{q} e^{-\beta \phi_{u u}(\widetilde{q})}\right\}=(N-M) \sum_{n=1}^{\infty} \frac{\widetilde{\rho}^{n}}{(n+1) !} \bar{C}_{n} .
$$

The first term in (4.15) can be obtained as a series in $\widetilde{\rho}$ by a procedure similar to that which lead us to (4.22), with a few notational differences. Our integrand now contains the coordinates of u-particles, which are integrated over, as well as the coordinates of r-particles, which are fixed. As in (4.16), we expand the integral as a series of products of Mayer $f$ functions, which we again depict as diagrams. There are now two varieties of nodes: those denoting u-particles, which we continue to represent as filled nodes $(\bullet)$, and those denoting 
r-particles, which we represent as open nodes ( o ). These will be referred to as "u-nodes" and "r-nodes", respectively. Lines still represent $f$-bonds, which may connect either two $\mathrm{u}$-nodes, or one r-node and one u-node. There are no bonds allowed between r-nodes in these diagrams, since we subtracted out all r-r interactions before arriving at the expression (4.15) for the correction term.

We must be careful in specifying the meaning of these new, more general diagrams. All diagrams (or components of diagrams) that consist solely of u-nodes retain their previous definitions. Diagrams or components that have one or more r-nodes, as well as one or more u-nodes, are defined similarly, except that they lack the prefactor $1 / V$, and the integrands are parameterized by the fixed coordinates associated with each r-node. Some examples:

$$
\begin{aligned}
& \longrightarrow=\int d \widetilde{q}_{1} f_{1, i} \\
& \overbrace{i}^{i}=\int d \widetilde{q}_{1} f_{1, i} f_{1, j} \\
& \overbrace{i}^{j}=\left(\frac{1}{V} \int d \widetilde{q}_{1} \int d \widetilde{q}_{2} f_{1,2}\right)\left(\int d \widetilde{q}_{1} \int d \widetilde{q}_{2} f_{1, i} f_{1, j} f_{2, j}\right)
\end{aligned}
$$

Note that the labels on r-nodes refer to specific particles, and thus may not be changed or omitted without altering the meaning of the diagram. An entirely unlabeled diagram with $p$ r-nodes is taken as shorthand for the sum over all topologically distinct ways of labelling that diagram using $p$ of the $M$ possible resolved indices, i.e.

$$
\begin{aligned}
& \longrightarrow=\sum_{i=1}^{M} i_{0} \longrightarrow \\
& \prod_{0}=\sum_{i<j}^{M}\left\{i_{i}^{0}+i_{j}^{j} j^{i}\right\}
\end{aligned}
$$

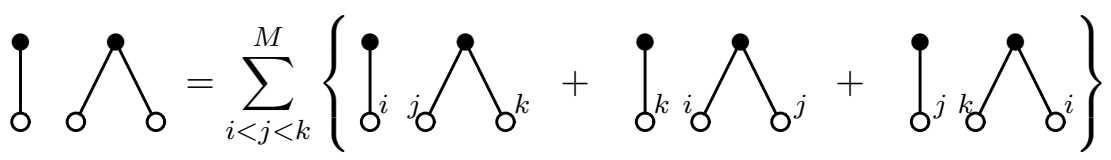

$$
\begin{aligned}
& \text { etc... }
\end{aligned}
$$

Using the above definitions, and by a counting argument nearly identical to the one that gave us (4.19) and (4.20), we obtain

$$
\frac{1}{V^{N-M}} \int d \widetilde{q} e^{-\beta\left[\phi_{r u}(\widehat{q}, \widetilde{q})+\phi_{u u}(\widetilde{q})\right]}=1+\sum_{n=1}^{N-M}\left(\frac{1}{V}\right)^{n} \Theta_{n}
$$

where

$$
\Theta_{n}=\sum_{\alpha \in P\{n\}} \sum_{\beta_{1}=0}^{M} \cdots \sum_{\beta_{k}=0}^{M} \frac{1}{\mu(\alpha, \beta)}\left(\begin{array}{c}
N-M \\
\alpha_{1}+\delta_{0, \beta_{1}}, \cdots, \alpha_{k}+\delta_{0, \beta_{k}}
\end{array}\right) \prod_{i=1}^{k} \mathbb{C}_{\beta_{i}, \alpha_{i}} .
$$


Here $\delta$ is the Kronecker delta function, $\beta_{i}$ is the number of r-nodes in the $i^{\text {th }}$ component of the diagram, and $\mu(\alpha, \beta)$ is again a multiplicity factor, though its definition has changed somewhat. It now contains a factor of $p$ ! for every $\alpha_{i}$ whose value is repeated $p$ times, provided the corresponding $\beta_{i}$ is zero. For example, $\mu((1,2,2,3,3,3),(6,0,0,7,0,0))=2$ ! 2 !. The $\mathbb{C}_{\ell, s}$ are a more general version of the $C_{s}$ introduced earlier. $\mathbb{C}_{\ell, s}$ is a weighted sum of all topologically distinct, connected, unlabeled diagrams consisting of $\ell$ r-nodes and $s$ u-nodes, where the weights are given by the number of distinct ways of arranging $s$ labels over the $\mathrm{u}$-nodes of the corresponding diagram, treating the r-nodes as distinguishable. Note that $\mathbb{C}_{0, s}=C_{s}$. The first few $\mathbb{C}_{\ell, s}$ with $\ell \geq 1$ are listed below:

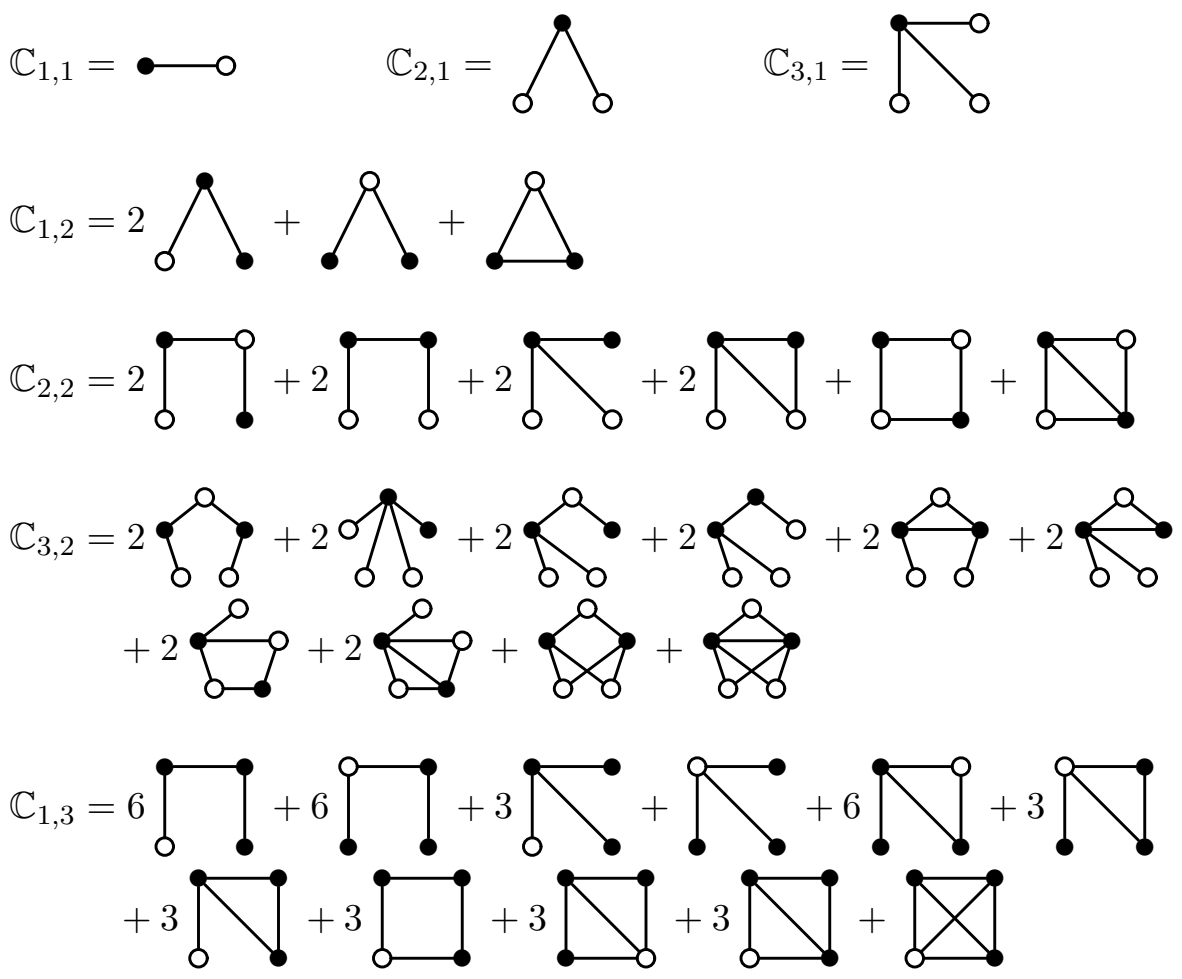

The next cluster sum in the sequence, $\mathbb{C}_{2,3}$, contains 42 diagrams, so we see that the length of $\mathbb{C}_{\ell, s}$ grows rapidly with $\ell$ and $s$.

Before we continue, we must generalize the definition of "reducible" introduced earlier. A connected diagram can be written as a product of smaller diagrams, and is thus reducible, if one or both of the following criteria are met:

(1) By removal of a single u-node, we can divide the diagram into two or more disconnected pieces, at least one of which contains no r-nodes.

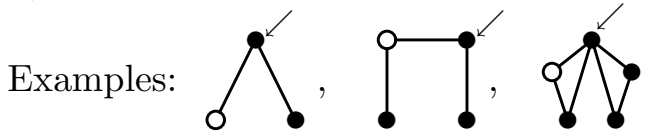

(2) By removal of some number of r-nodes, we can divide the diagram into two or more disconnected pieces.

Examples:
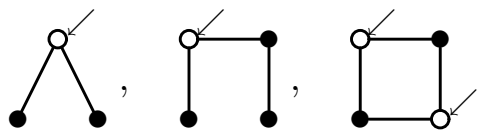
For diagrams with only u-nodes, this is equivalent to the previous definition. We define $\overline{\mathbb{C}}_{\ell, s}$ as the irreducible part of $\mathbb{C}_{\ell, s}$. The $\overline{\mathbb{C}}_{\ell, s}$ corresponding to the $\mathbb{C}_{\ell, s}$ listed above are:

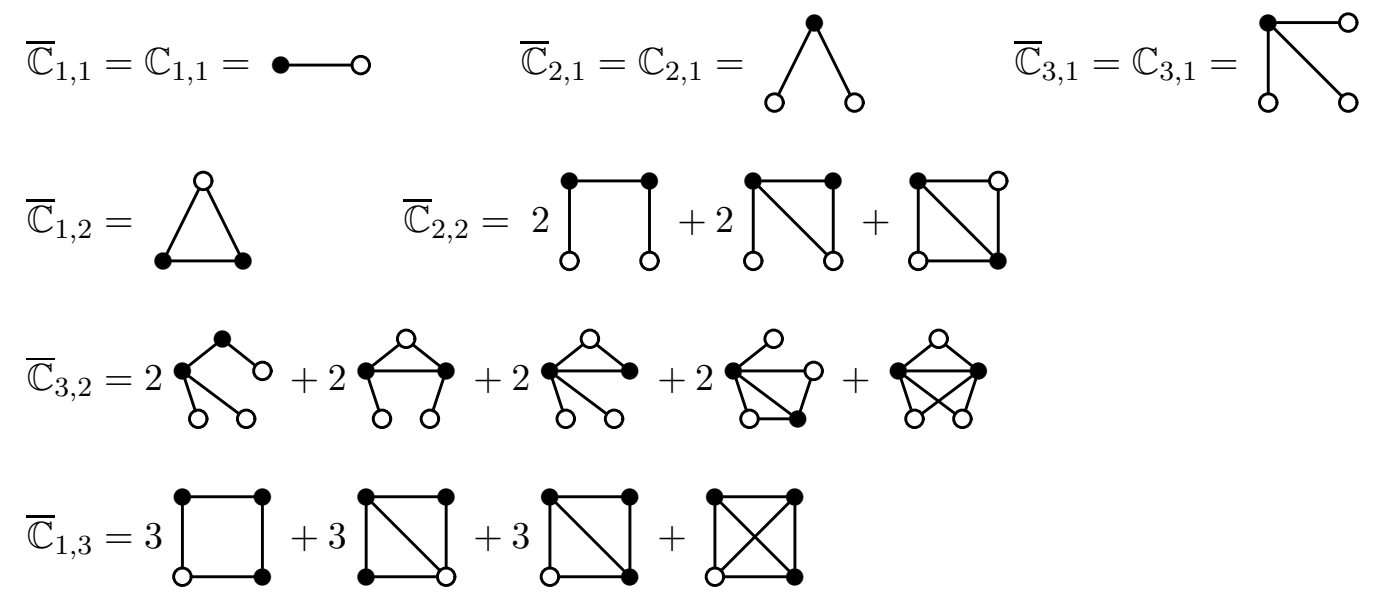

As was done previously, we Taylor expand the logarithm of (4.23) in powers of $1 / V$ to obtain

$$
\begin{aligned}
\log \left\{\frac{1}{V^{N-M}} \int d \widetilde{q} e^{-\beta\left[\phi_{r u}(\widehat{q}, \widetilde{q})+\phi_{u u}(\widetilde{q})\right]}\right\} & =\log \left\{1+\sum_{n=1}^{N-M}\left(\frac{1}{V}\right)^{n} \Theta_{n}\right\} \\
& =\sum_{n=1}^{\infty}\left(\frac{1}{V}\right)^{n} \Psi_{n} .
\end{aligned}
$$

By comparing the expansions of both sides of (4.25) term by term, using the definitions of the $\overline{\mathbb{C}}_{\ell, s}$, and passing to the thermodynamic limit, we find that the first few $\Psi_{n}$ satisfy

$$
\Psi_{n}=\frac{(N-M)^{n+1}}{(n+1) !} \bar{C}_{n}+\frac{(N-M)^{n}}{n !} \sum_{\ell=1}^{M} \overline{\mathbb{C}}_{\ell, n} .
$$

As before, this relation holds generally, but the proof is nontrivial, and is beyond the scope of this thesis. A proof based in the grand canonical ensemble, which is equivalent in the thermodynamic limit, can be found in [23] and the references therein.

Using (4.22), (4.25), and (4.26), we now arrive at the following expression for the correction term (4.15):

$$
\log \left\{\frac{\int d \widetilde{q} e^{-\beta\left[\phi_{\text {ru }}(\widehat{q}, \widetilde{q})+\phi_{u u}(\widetilde{q})\right]}}{\int d \widetilde{q} e^{-\beta \phi_{u u}(\widetilde{q})}}\right\}=\sum_{n=1}^{\infty} \frac{1}{n !} \widetilde{\rho}^{n} \sum_{\ell=1}^{M} \overline{\mathbb{C}}_{\ell, n},
$$

After combining (4.27) with (4.14), and performing a simple regrouping of terms, we obtain our final expression for the renormalized Hamiltonian:

$$
\mathscr{H}(\widehat{q}, \widehat{p})=\sum_{i=1}^{M} \frac{\left|\widehat{p}_{i}\right|^{2}}{2 m}+\widehat{\phi}(\widehat{q})+F(N-M, \widetilde{\rho}, T),
$$

where the $F(N-M, \widetilde{\rho}, T)$ term is a constant that has no effect on the dynamics, and is included for completeness only. Here

$$
\widehat{\phi}(\widehat{q})=\widehat{\phi}^{(1)}(\widehat{q})+\widehat{\phi}^{(2)}(\widehat{q})+\widehat{\phi}^{(3)}(\widehat{q})+\cdots
$$


is called the renormalized potential. $\widehat{\phi}^{(\ell)}$ represents a sum of $\ell$-particle interactions, and is given by

$$
\widehat{\phi}^{(\ell)}(\widehat{q})=\delta_{\ell, 2} \phi_{r r}(\widehat{q})-k_{B} T \sum_{n=1}^{\infty} \frac{1}{n !} \widetilde{\rho}^{n} \overline{\mathbb{C}}_{\ell, n} .
$$

The fact that the original pair potential gains 3-particle, 4-particle, and higher interactions after renormalization should come as no surprise to those familiar with other, similar problems in physics, such as the block renormalization of spin systems. We will discuss the convergence properties of the sum (4.30) in Section 4.2.3.

Let us examine the various terms in (4.29). The first term in the renormalized potential is

$$
\begin{aligned}
& \widehat{\phi}^{(1)}(\widehat{q})=-k_{B} T \sum_{n=1}^{\infty} \frac{1}{n !} \widetilde{\rho}^{n} \overline{\mathbb{C}}_{1, n} \\
& =-k_{B} T\left(\tilde{\rho}\{\bullet \bullet\}+\frac{1}{2 !} \widetilde{\rho}^{2}\{\stackrel{\Omega}{\varrho}\}\right.
\end{aligned}
$$

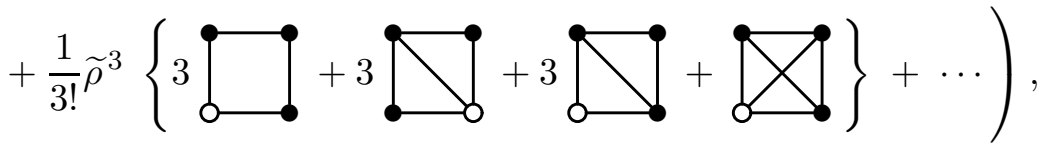

which is a single-particle effective field felt by each r-particle due to the surrounding sea of $\mathrm{u}$-particles (recall that each of the diagrams above is shorthand for a sum over all $M$ possible labellings of the single r-node). However, unless an $\mathrm{r}$-particle is within a few multiples of $\sigma$ of the boundary $\partial \Omega$, spatial isotropy implies that all of the diagrams in the expansion of $\widehat{\phi}^{(1)}$ are constants, independent of the resolved coordinates. The r-node is merely an anchor that serves as the origin for the integrals associated with the u-nodes. In fact, a simple counting argument can be used to show that, in general, $\mathbb{C}_{1, n}=M C_{n}$, in which case

$$
\widehat{\phi}^{(1)}=-k_{B} T M \sum_{n=1}^{\infty} \frac{1}{n !} \widetilde{\rho}^{n} \bar{C}_{n}=\text { constant. }
$$

In the event that one of the r-particles should happen to be within several $\sigma$-multiples of a wall, the corresponding single-particle field $\widehat{\phi}^{(1)}$ would be nonconstant due to the anisotropy of space in this region, and the particle would feel a net force which, by symmetry, must be directed normal to the wall. The magnitude and sign of this force would depend on the distance to the wall, as well as upon the shape of the interparticle potential. In principle, by appropriately restricting the integration domains of the diagrams to reflect the proximity of the wall, (4.31) could be used to estimate the potential associated with this force. Since both the underlying interparticle potential $U(r)$ and the correspoding Mayer function $f(r)$ have a short range equal to several multiples of $\sigma$, we expect these "boundary forces" to persist for a comparable distance into the fluid from the wall. However, since we are ignoring boundary effects, $\widehat{\phi}^{(1)}$ is assumed to be constant for all particles.

The second term in the renormalized potential consists of diagrams with two r-nodes and any number of u-nodes. By spatial isotropy, the associated integrals can depend only on the distance between the two r-particles. Thus we can write $\widehat{\phi}^{(2)}$ as

$$
\widehat{\phi}^{(2)}(\widehat{q})=\sum_{i<j}^{M} \widehat{U}\left(\left|\widehat{q}_{i}-\widehat{q}_{j}\right|\right),
$$



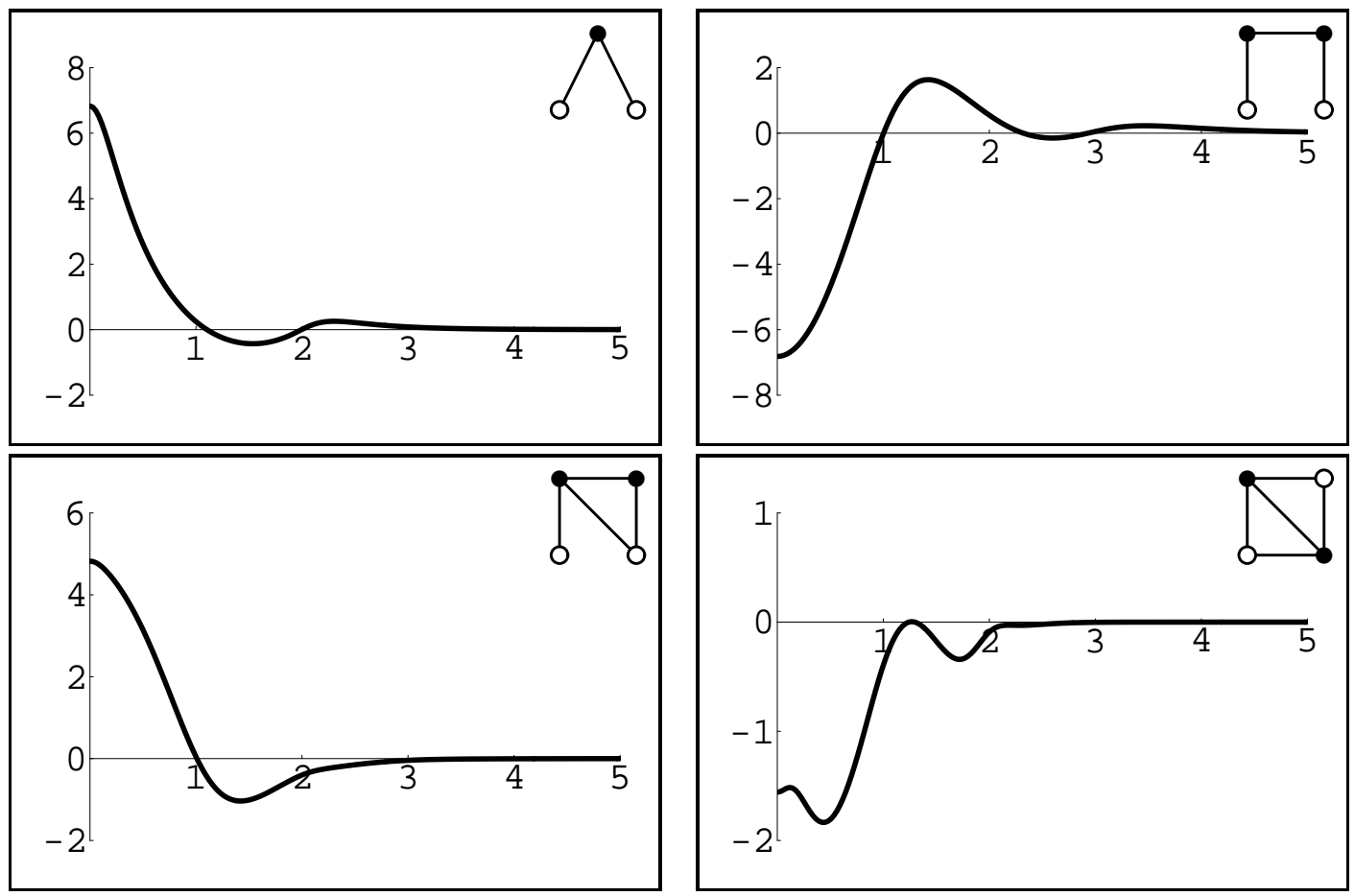

Figure 4.1: Diagrams contributing to the first and second order corrections to the LennardJones potential at $T^{*}=1.6$, in reduced units.

where $\widehat{U}$, the renormalized pair potential, is given by

$$
\begin{aligned}
& \widehat{U}\left(\left|\widehat{q}_{i}-\widehat{q}_{j}\right|\right)=U\left(\left|\widehat{q}_{i}-\widehat{q}_{j}\right|\right)
\end{aligned}
$$

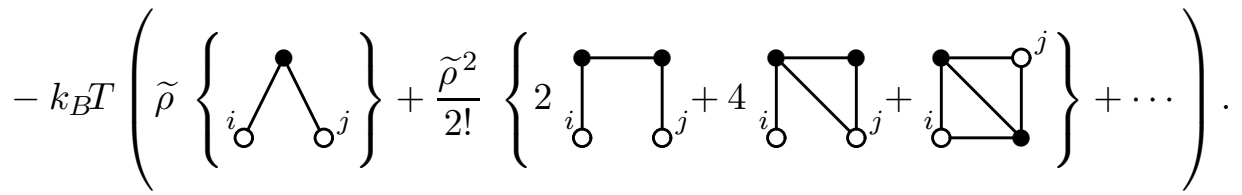

In arriving at (4.32), we have used the fact that, by symmetry,

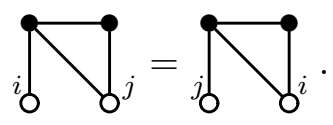

The various diagrams that make up the correction terms to the "bare potential" $U$ must in general be calculated numerically for each value of the temperature. In order to compute $\widehat{U}$ up through order $n$ in $\widetilde{\rho}$, we need to evaluate several $n d$-dimensional integrals of products of $f$-functions for a large number of values of the interparticle separation. This is computationally intensive, however, and in practice, we will truncate (4.32) after the second order correction. To give some idea of what these corrections look like, the first four diagrams in (4.32) are plotted in Figure 4.1 as a function of the interparticle separation for the Lennard-Jones potential in three dimensions at $T^{*}=1.6$.

The renormalized pair potential $\widehat{U}$ can also be calculated exactly from a MD simulation, using methods to be described in Section 4.2.4. The results of such calculations for several densities are shown in Figure 4.2, along with $\widehat{U}$ obtained by truncating (4.32) after the term 

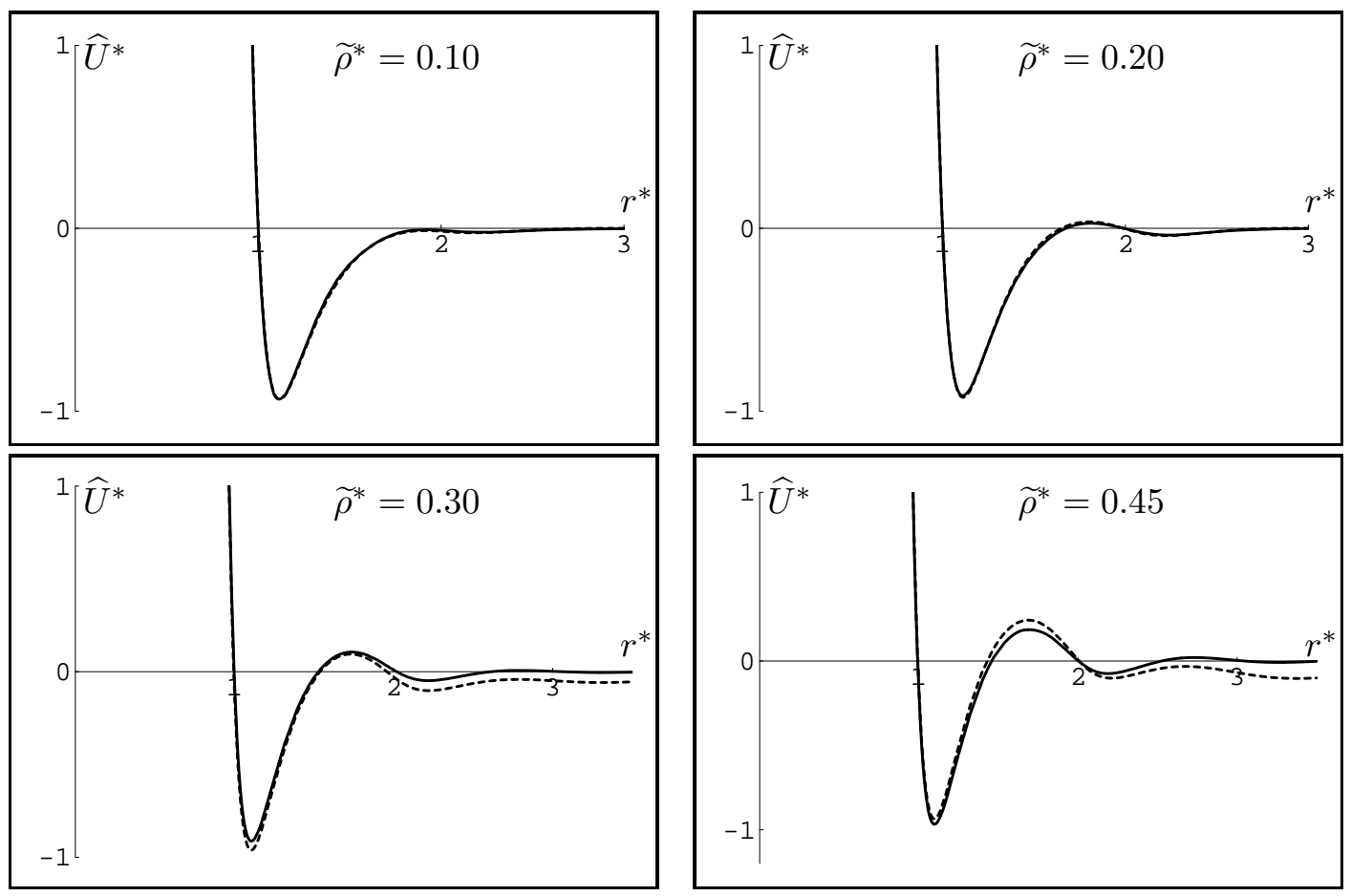

Figure 4.2: Solid lines: $\widehat{U}^{*}\left(r^{*}\right)$ obtained from MD experiments. Dashed lines: $\widehat{U}^{*}\left(r^{*}\right)$ obtained by truncating the diagrammatic series (4.32) to second order. Here $T^{*}=1.6$.

second order in $\widetilde{\rho}$. We see that the truncated series works well up to about $\widetilde{\rho}^{*} \sim 0.30$, at which point significant errors begin to appear for $r^{*} \gtrsim 2.0$. This occurs because the higher order terms in (4.32) that we have neglected determine the shape of $\widehat{U}(r)$ for large values of $r$.

Note that as the density $\widetilde{\rho}$ of u-particles increases, the renormalized pair potential $\widehat{U}$ develops features that are not present in the bare potential $U$. As we will see in Section 4.2.4, this effect becomes even more pronounced at higher densities. The physical reason for this is that as $\widetilde{\rho}$ increases, it becomes more and more probable for one or more u-particles to occupy the region between any two r-particles. As the two r-particles approach one another, they feel a resistance due to the need to push the interceding u-particles out of the way. The average effect of this is to create a series of additional maxima and minima in $\widehat{U}$. For the Lennard-Jones potential, particles have an effective "size" of roughly $\sigma$. Two r-particles that are a distance $n \sigma$ apart therefore have room for about $n$ u-particle between them. For this reason, we expect successive maxima in $\widehat{U}$ to be separated by a distance of approximately $\Delta r \approx \sigma$, or $\Delta r^{*} \approx 1$ in reduced units. Though this effect is hard to discern in Figure 4.2, it can can be seen much more easily for higher values of $\widetilde{\rho}$ (see, in particular, Figure 4.4). 
The next term in (4.29) is the triplet potential

$$
\begin{aligned}
& \widehat{\phi}^{(3)}(\widehat{q})=-k_{B} T \sum_{n=1}^{\infty} \frac{1}{n !} \widetilde{\rho}^{n} \overline{\mathbb{C}}_{3, n} \\
& =-k_{B} T(\tilde{\rho}\{\overbrace{0}^{\circ}\}
\end{aligned}
$$

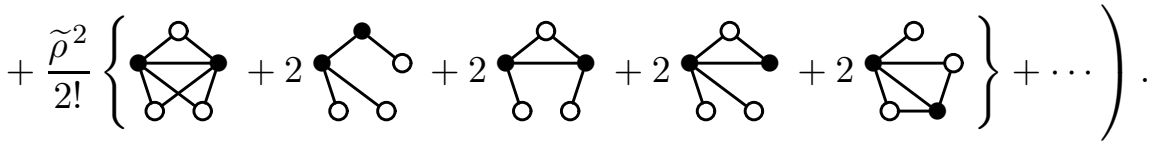

Since the distances between the three particles serve to uniquely specify their relative positions, spatial isotropy allows us to express this as

$$
\widehat{\phi}^{(3)}(\widehat{q})=\sum_{i<j<k}^{M} \widehat{U}_{3}\left(\left|\widehat{q}_{i}-\widehat{q}_{j}\right|,\left|\widehat{q}_{j}-\widehat{q}_{k}\right|,\left|\widehat{q}_{k}-\widehat{q}_{i}\right|\right),
$$

where $\widehat{U}_{3}$ must be invariant under arbitrary permutations of its three arguments. For numerical applications, the force on particle $i$ due to the triplet potential can, in principle, be written as

$$
\begin{aligned}
F_{i}^{(3)}(\widehat{q}) & =-\nabla_{\widehat{q}_{i}} \sum_{\substack{j<k \\
j, k \neq i}}^{M} \widehat{U}_{3}\left(\left|\widehat{q}_{i}-\widehat{q}_{j}\right|,\left|\widehat{q}_{j}-\widehat{q}_{k}\right|,\left|\widehat{q}_{k}-\widehat{q}_{i}\right|\right) \\
& =\sum_{\substack{j<k \\
j, k \neq i}}^{M}\left\{\left(\widehat{q}_{i}-\widehat{q}_{j}\right) \widehat{\omega}_{3}\left(\left|\widehat{q}_{i j}\right| ;\left|\widehat{q}_{i k}\right|,\left|\widehat{q}_{j k}\right|\right)+\left(\widehat{q}_{i}-\widehat{q}_{k}\right) \widehat{\omega}_{3}\left(\left|\widehat{q}_{i k}\right| ;\left|\widehat{q}_{i j}\right|,\left|\widehat{q}_{j k}\right|\right)\right\},
\end{aligned}
$$

where $\widehat{q}_{i j}=\widehat{q}_{i}-\widehat{q}_{j}$, and

$$
\widehat{\omega}_{3}\left(x_{1} ; x_{2}, x_{3}\right) \equiv-\frac{1}{x_{1}} \frac{\partial}{\partial x_{1}} \widehat{U}_{3}\left(x_{1}, x_{2}, x_{3}\right)
$$

is the three-particle analog of the $\omega$ function defined in Section 3.4.4. In practice, however, the calculation of the various terms in the expansion of the triplet potential is too great a computational challenge to be useful. For example, in order to evaluate $\widehat{U}_{3}$ by discretising the range of each of its three arguments into 100 segments (which amounts to a very low resolution), one must calculate and store on the order of $10^{6}$ separate high-dimensional integrals for each diagram. For this reason, we are forced to neglect the triplet and all higher potentials, and approximate the renormalized potential by its pair contribution (4.32) alone. At low densities, this approximation is plausible, since the multiple-particle interactions that are mediated by the neglected potentials would not be expected to occur with any significant frequency until the density becomes somewhat higher.

\subsubsection{The General Case: $M$-particle Distribution Functions}

The $\widetilde{\rho}$-expansion of the renormalized potential (4.30) is an example of a virial series, in which a thermodynamic or structural quantity is expanded as a series in the density. Virial series 
have historically played an important role in the theory of non-ideal gases and low density liquids [41]. However, their utility for obtaining useful results is limited to low densities. For this reason, we need to explore alternative methods of evaluating the renormalized potential that are valid at higher densities.

A comparison between (4.11) and (4.28) reveals that

$$
e^{-\beta \widehat{\phi}(\widehat{q})}=\frac{\int d \widetilde{q} e^{-\beta \phi(q)}}{\int d \widetilde{q} e^{-\beta \phi_{u u}(\widetilde{q})}}
$$

which we will take as the definition of the renormalized potential. We may now relate $\widehat{\phi}$ to the $M$-particle distribution function $g^{(M)}$ commonly encountered in statistical mechanics, which, for a particular form of the interparticle interaction, is defined as

$$
g^{(M)}(\widehat{q})=\frac{\left(\begin{array}{c}
\text { Probability of the configuration } \\
\widehat{q} \text { in the system with interactions }
\end{array}\right)}{\left(\begin{array}{c}
\text { Probability of the same } \\
\text { configuration in an ideal gas }
\end{array}\right)} .
$$

It can be shown [23] that this is equivalent to

$$
g^{(M)}(\widehat{q}, \widetilde{\rho}, T) \equiv \frac{1}{\widetilde{\rho}^{M}} \frac{N !}{(N-M) !} \frac{\int d \widetilde{q} e^{-\beta \phi(q)}}{\int d q e^{-\beta \phi(q)}},
$$

where the dependence of $g^{(M)}$ on the density $\widetilde{\rho}$ of particles whose coordinates have been integrated away, as well as on the temperature, has been made explicit. Combining (4.33) and (4.35) yields

$$
\widehat{\phi}(\widehat{q})=-k_{B} T \log \left(g^{(M)}(\widehat{q}, \widetilde{\rho}, T)\right)-\underbrace{k_{B} T \log \left(\rho^{M} \frac{(N-M) !}{N !} \frac{\int d q e^{-\beta \phi(q)}}{\int d \widetilde{q} e^{-\beta \phi_{u u}(\widetilde{q})}}\right)}_{\text {an irrelevant constant }},
$$

which is valid at any density or temperature, and is the most general expression for the renormalized potential.

The $M$-particle distribution function is often written as $g^{(M)}=\exp (-\beta \psi)$, where $\psi$ is called the potential of mean force. Equation (4.36) tells us that $\widehat{\phi}=\psi+$ constants. In what follows, we will ignore all constants, and treat $\psi$ and $\widehat{\phi}$ as equal. Just as in (4.29), the potential of mean force $\psi$, and hence also the renormalized potential, can be written as the sum of a pair contribution, a triplet contribution, and so on. For example, the twoparticle and three-particle distribution functions are defined in terms of the pair and triplet potentials as

$$
g^{(2)}(r, \widetilde{\rho}, T) \equiv \exp [-\beta \widehat{U}(r)]
$$

and

$$
g^{(3)}\left(r_{1}, r_{2}, r_{3}, \widetilde{\rho}, T\right) \equiv \exp \left[-\beta\left(\widehat{U}\left(r_{1}\right)+\widehat{U}\left(r_{2}\right)+\widehat{U}\left(r_{3}\right)+\widehat{U}_{3}\left(r_{1}, r_{2}, r_{3}\right)\right)\right] .
$$

Higher many-particle distribution functions are defined analogously.

The two-particle distribution function $g^{(2)}(r, \widetilde{\rho}, T)$ is usually refered to as the radial distribution function (RDF), and has been extensively studied. It describes the distribution of particles around a single particle located at the origin in a fluid at the state point $(\widetilde{\rho}, T)$. 
For a fluid with no interparticle interactions, $g^{(2)}(r)=1$ for all $r$. In a real fluid, any two particles are prevented from approaching one another closely by a hard-core repulsion, and $g^{(2)}(r) \rightarrow 0$ as $r \rightarrow 0$. However, as $r \rightarrow \infty$, the two particles become uncorrelated, and $g^{(2)} \rightarrow 1$. In the intermediate region, which is usually several multiples of $\sigma$ wide, the RDF tends to oscillate, though the extent of the oscillation depends strongly on the density and temperature. Given $g^{(2)}$, we can calculate the renormalized pair potential as $\widehat{U}(r)=-k_{B} T \log \left(g^{(2)}(r)\right)$.

\subsubsection{Convergence of the Virial Expansion}

Now that we have connected the renormalized potential $\widehat{\phi}$ to the $M$-particle distribution function $g^{(M)}$ familiar from statistical mechanics, we can use several classical results to investigate the convergence properties of the series (4.30). As was originally shown by Mayer and Montroll [42], $g^{(M)}(\widehat{q}, \widetilde{\rho}, T)$ has the following series expansion in the density $\widetilde{\rho}$ :

$$
g^{(M)}(\widehat{q}, \widetilde{\rho}, T)=e^{-\beta \phi_{r r}(\widehat{q})}\left(1+\sum_{n=1}^{\infty} \frac{1}{n !} \widetilde{\rho}^{n} \sum_{\ell=1}^{M} \mathbb{C}_{\ell, n}\right)
$$

If we recall that (to within constants) $\widehat{\phi}(\widehat{q})=-k_{B} T \log \left(g^{(M)}(\widehat{q})\right)$, then we can write

$$
\widehat{\phi}(\widehat{q})=\phi_{r r}(\widehat{q})-k_{B} T \log \left(1+\sum_{n=1}^{\infty} \frac{1}{n !} \widetilde{\rho}^{n} \sum_{\ell=1}^{M} \mathbb{C}_{\ell, n}\right) .
$$

At the same time, combining equations (4.29) and (4.30) allows us to write the renormalized potential as

$$
\widehat{\phi}(\widehat{q})=\phi_{r r}(\widehat{q})-k_{B} T \sum_{n=1}^{\infty} \frac{1}{n !} \widetilde{\rho}^{n} \sum_{\ell=1}^{M} \overline{\mathbb{C}}_{\ell, n} .
$$

Comparing (4.39) and (4.38) shows that the effect of the log operation in (4.38) is simply to remove all of the reducible graphs from the sum. This is the primary result of [42].

The maximum value of $\widetilde{\rho}$ for which the series in (4.37) and (4.39) converge is called the radius of convergence of these series, and it represents the largest value of $\widetilde{\rho}$ for which the virial expansion of the renormalized potential is useable and valid. Lebowitz and Penrose [34] have demonstrated that, for $s \geq 1$, the virial series of both the $s$-particle distribution function $g^{(s)}\left(q_{1}, \ldots, q_{s}\right)$ and the $s$-particle potential of mean force $\psi\left(q_{1}, \ldots, q_{s}\right)$ have a radius of convergence that is at least as great that of the virial series of the pressure $P$, which is given by [41]

$$
P(\rho)=\rho k_{B} T\left(1-\sum_{n=1}^{\infty} \frac{n}{n+1} \frac{\rho^{n}}{n !} \bar{C}_{n}\right) .
$$

This means that a lower bound on the radius of convergence of (4.40) will also serve as a lower bound on the convergence of the series in (4.30) for any value of $\ell$. This is advantageous because (4.40) is much simpler to work with than either (4.37) or (4.39), and many rigorous results concerning this series have been derived over the years. 
We define the class of stable potentials as the set of all interparticle pair potentials $U(r)$ that satisfy the following two properties:

$$
\begin{aligned}
& A \equiv \int d x\left|e^{-\beta U(|x|)}-1\right|<\infty \\
& \sum_{i<j}^{N} U\left(\left|x_{i}-x_{j}\right|\right) \geq-N B, \text { for some constant } B \geq 0
\end{aligned}
$$

Condition (4.41) is obeyed by any potential that satisfies the assumptions we made about the form of $U(r)$ in Section 3.1. Furthermore, Ruelle [54] has proven that (4.42) is satisfied for a wide range of potentials, including the Lennard-Jones potential. We now define $\rho_{o}$ as the radius of convergence of (4.40). Several lower bounds on $\rho_{o}$ have been derived (see [55] or [33] for a summary of these results), and the one we will use is that of Groeneveld [22], which states that

$$
\rho_{o} \geq \rho_{o}^{-} \equiv \frac{1}{e A\left(1+e^{2 \beta B}\right)} .
$$

Given a particular potential, the integral $A$ is easily calculated numerically. Finding a value for $B$ is often much more difficult. In the case of the Lennard-Jones potential (3.3) we can determine $B$ by noting that, given $N \gg 1$ particles, the configuration of lowest potential energy occurs when the particles are arrayed in a face-centered cubic (FCC) lattice. If we assume that the FCC cell has a side of length " $a$ ", then the positions of the particles on the corners of each cell in this lattice can be written as $a \times(i, j, k)$, whereas the positions of the particles on the faces of each cell can be written as $a \times\left(i+\frac{1}{2}, j+\frac{1}{2}, k\right), a \times\left(i+\frac{1}{2}, j, k+\frac{1}{2}\right)$, and $a \times\left(i, j+\frac{1}{2}, k+\frac{1}{2}\right)$. Here $i, j$, and $k$ are integers that range from $-\infty$ to $\infty$. Since all particles are equivalent, we can focus on the particle at the origin $(i=j=k=0)$ without loss of generality. The potential energy due to this particle's interactions with all of the other particles in the lattice is then the same as the potential energy per particle in the system as a whole. This quantity is a function of the lattice spacing $a$, and can be written as

$$
\frac{\phi(a)}{N}=2 \epsilon\left[\frac{\sigma^{12}}{a^{12}}\left(\alpha_{12}+3 \beta_{12}\right)-\frac{\sigma^{6}}{a^{6}}\left(\alpha_{6}+3 \beta_{6}\right)\right],
$$

where

$$
\begin{aligned}
& \alpha_{n} \equiv \sum_{i, j, k=-\infty}^{\infty} \frac{1}{\left(i^{2}+j^{2}+k^{2}\right)^{n / 2}}, \\
& \beta_{n} \equiv \sum_{i, j, k=-\infty}^{\infty} \frac{1}{\left[(i+1 / 2)^{2}+(j+1 / 2)^{2}+k^{2}\right]^{n / 2}} .
\end{aligned}
$$

In the expression for $\alpha_{n}$, the ' notation indicates that the $i=j=k=0$ term is not included in the sum. In order to calculate $B$, we must find the finimum of (4.44) over all values of $a$. Using the fact that $\alpha_{6}=8.402, \alpha_{12}=6.202, \beta_{6}=35.743$, and $\beta_{12}=256.746$, it is easy to show that ${ }^{2}$

$$
B=-\min _{a} \frac{\phi(a)}{N}=8.610 \epsilon
$$

\footnotetext{
${ }^{2}$ It should be noted that Ruelle's expression for $B$ in [54] yields $B=6258.27 \epsilon$ for the Lennard-Jones potential, which is a gross over-estimate.
} 


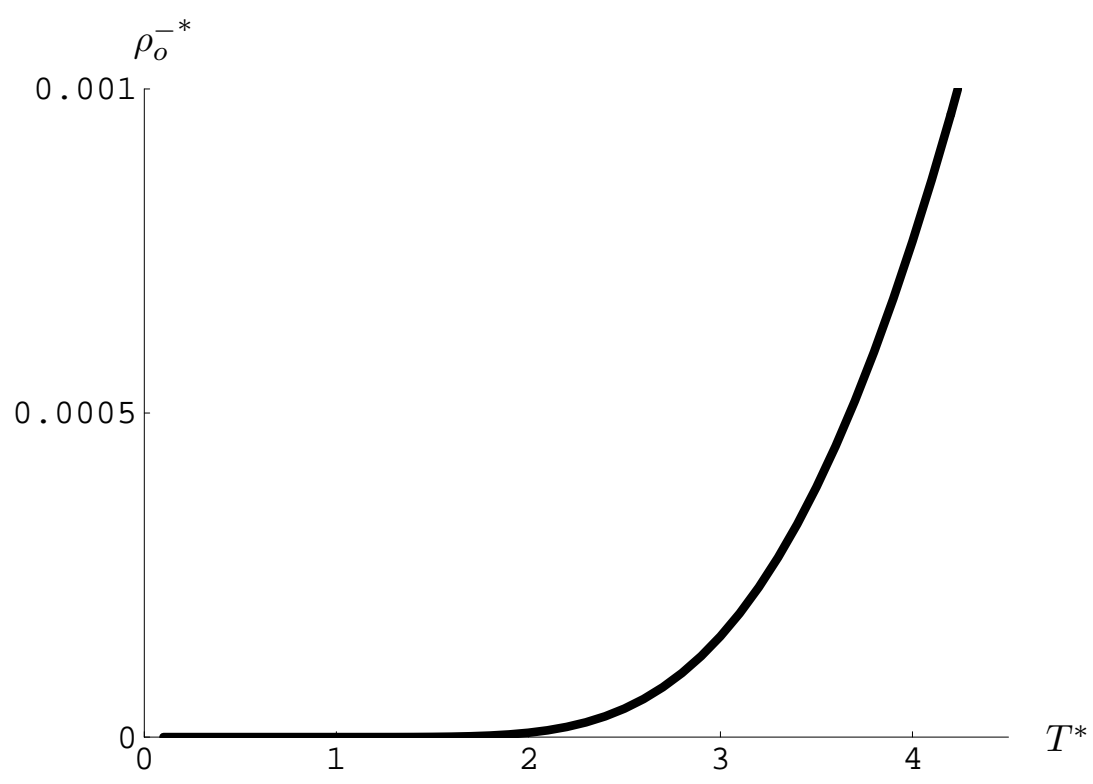

Figure 4.3: Groeneveld's lower bound $\rho_{o}^{-*}$, plotted in reduced units as a function of $T^{*}$ for the Lennard-Jones potential.

Groeneveld's lower bound $\rho_{o}^{-}$on the radius of convergence $\rho_{o}$ is plotted for the LennardJones potential in Figure 4.3. We see that the typical values of $\rho_{o}^{-}$are extremely low. In particular, for $T^{*}=1.6$, the value of the temperature used in numerics throughout this thesis, we have $\rho_{o}^{-*} \sim 6.5 \times 10^{-7}$. However, we should keep in mind that there was no reason a priori to assume that $\rho_{o}$ was different from zero. In other words, the series (4.40) might not have converged for any value of $\rho$. It is therefore encouraging that one can prove convergence for nonzero density. Furthermore, (4.43) is only a lower bound, and the true value of $\rho_{o}$ may be many orders of magnitude higher. In fact, numerical studies of the virial series for the pressure $[65,23]$ have shown empirically that $(4.40)$, and hence also (4.30), converges at densities that extend far into the dense fluid range (i.e. $\left.\rho_{o}^{*} \gtrsim 0.7\right)$. This is corroborated by Figure 4.2, which suggests that keeping only two terms in the expansion (4.32) is sufficient to capture the general shape of $\widehat{U}$ at densities up to $\widetilde{\rho}^{*} \sim 0.5$.

\subsubsection{Calculating the Renormalized Pair Potential}

There are various methods of calculating $g^{(2)}$ for a given state point. At low densities, one can use the diagrammatic expansion of the previous section to find $g^{(2)}$ via $\widehat{U}$. At higher densities, there are several approximate and exact integral equations for $g^{(2)}$ that can be solved numerically. These will not be described here. The RDF can also be determined experimentally, either by computer simulation using $\mathrm{MD}$, as in [66], or by direct X-ray scattering experiments on real fluids (see, for example, [21]).

In this thesis, we will derive our high-density RDF's (and the corresponding $\widehat{U}$ 's) from 
computer MD data. The definition (4.34) of $g^{(M)}$ allows us to write $g^{(2)}$ as

$$
g^{(2)}(r)=\frac{\left(\begin{array}{c}
\text { Number of particle pairs separated } \\
\text { by a distance between } r \text { and } r+d r \\
\text { in the system with interactions }
\end{array}\right)}{\left(\begin{array}{c}
\text { Number of particle pairs separated } \\
\text { by the same distance in an ideal gas }
\end{array}\right)},
$$

where " $d r$ " is a small interval. Thus $g^{(2)}(r, \widetilde{\rho}, T)$ is easily evaluated by running a MD simulation of the full system (3.4) with $N$ particles at density $\widetilde{\rho}$ and temperature $T$. The numerator in (4.45) can be found directly by performing an operation every few timesteps in which all $N(N-1) / 2$ of the interparticle distances are sorted into bins and counted [3]. Examples of $g^{(2)}(r)$ calculated in this fashion, as well as the corresponding $\widehat{U}$ 's, are plotted in Figure 4.4 for several moderate-to-high values of $\widetilde{\rho}$. Note that the prediction made earlier that the successive maxima of $\widehat{U}$ should be separated by a distance of $\Delta r \approx \sigma\left(\Delta r^{*} \approx 1\right.$ in reduced units) is verified by these high-density results.

The three-particle distribution function $g^{(3)}$, can, in principle, be determined from computer simulation or real experiments (for an example of the latter for a colloidal liquid in two dimensions, see [67]). In reality, the problem of extracting $g^{(3)}$ from simulation data is much more difficult than that of determining $g^{(2)}$, for essentially the same reasons as in the low-density diagrammatic case. We therefore choose to ignore the triplet and all higher potentials, and again approximate $\widehat{\phi}$ by its pairwise term alone:

$$
\widehat{\phi}(\widehat{q}) \approx \widehat{\phi}^{(2)}(\widehat{q})
$$

For arbitrary densities, (4.46) is a rather drastic approximation, and it seems plausible to expect it to break down as $\widetilde{\rho}$ increases and the magnitude of the many-particle contributions to the renormalized potential becomes large. The reality of when and why this approximation fails is more subtle, however, as will be discussed in Section 4.4.

\subsection{The Relationship Between the Full and Reduced Systems}

We now have an approximate model for the evolution of the resolved coordinates, in the form of a dynamical system governed by the renormalized Hamiltonian (4.28):

$$
\begin{aligned}
\frac{d}{d t} \bar{q}_{i} & =\frac{1}{m} \bar{p}_{i} & \bar{q}_{i}(0) & =\widehat{x}_{i} \\
\frac{d}{d t} \bar{p}_{i} & =-\nabla_{\bar{q}_{i}} \widehat{\phi}(\bar{q}) & \bar{p}_{i}(0) & =\widehat{y}_{i},
\end{aligned}
$$

where $1 \leq i \leq M$. As described above, we will generally ignore all but the pair contribution to the renormalized potential:

$$
\widehat{\phi}(\bar{q}) \approx \sum_{i<j}^{M} \widehat{U}\left(\left|\bar{q}_{i}-\bar{q}_{j}\right|\right)
$$

Here $\widehat{U}$ may be calculated from the diagrammatic expansion (4.32) at low unresolved densities, and from MD data for higher densities.

Our goal is to determine how the macroscopic properties of the full system (as described in Section 3.3), can be extracted from the dynamics of (4.47). We begin by considering 

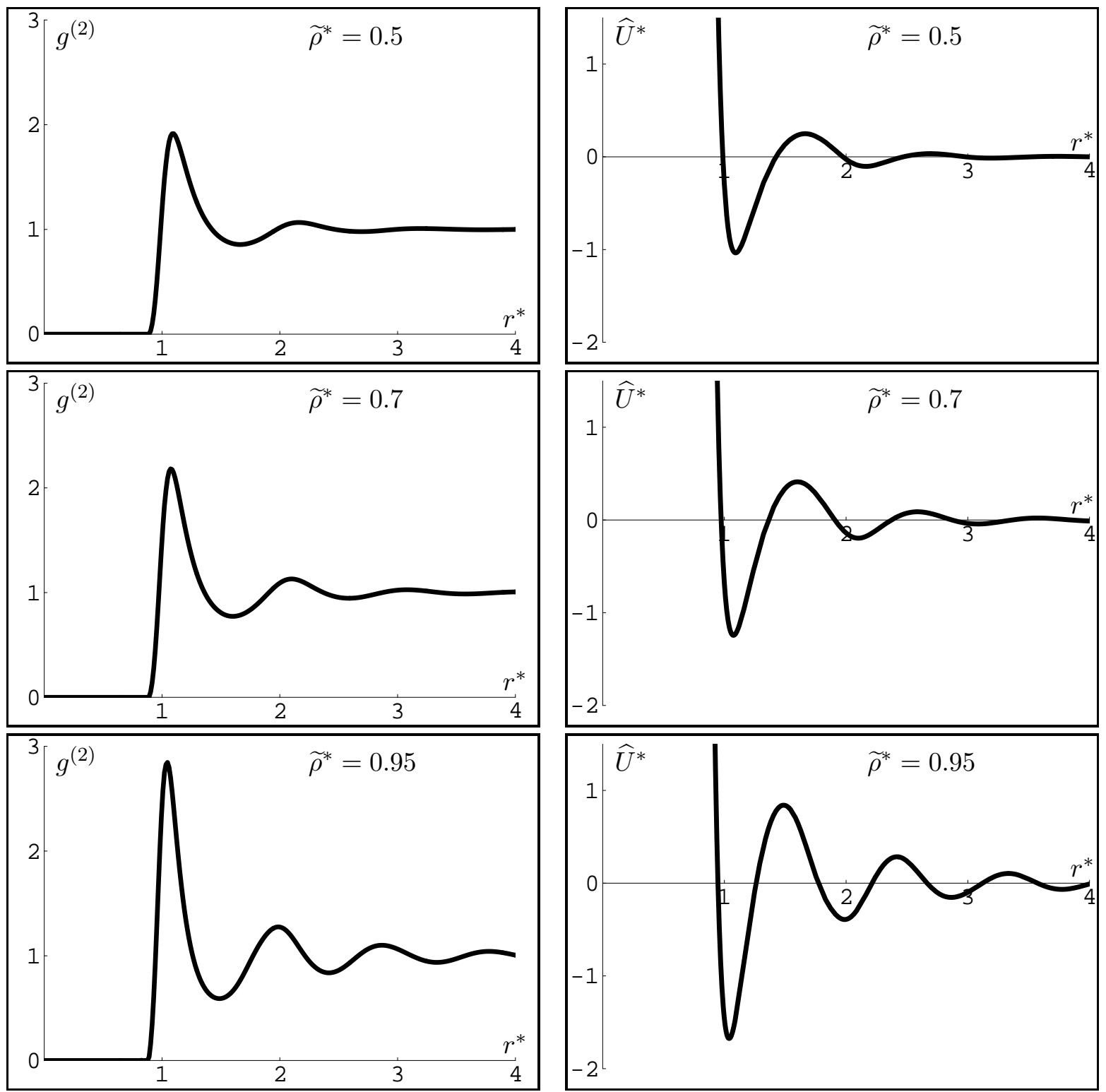

Figure 4.4: $g^{(2)}\left(r^{*}\right)$ and $\widehat{U}^{*}\left(r^{*}\right)$ of the Lennard-Jones potential determined by direct MD simulation for several values of $\widetilde{\rho}^{*}$. Here $T^{*}=1.6$. 
(4.47), with the exact expression (4.33) for $\widehat{\phi}$. Under the influence of a heat bath in real systems, or a thermostat on the computer ${ }^{3},(4.47)$ will quickly equilibrate to the renormalized canonical distribution (RCD):

$$
\hat{f}_{c}(\widehat{q}, \widehat{p})=\frac{1}{\widehat{Z}} e^{-\beta \mathscr{H}(\widehat{q}, \widehat{p})},
$$

where $\widehat{Z}=\int d \widehat{p} \int d \widehat{q} \exp (-\beta \mathscr{H}(\widehat{q}, \widehat{p}))$. Given some phase variable $A$ which is a function of the resolved coordinates alone (a "resolved phase variable"), we will write its average under the RCD as

$$
\langle A\rangle_{R C} \equiv \frac{\int d \widehat{p} \int d \widehat{q} A(\widehat{q}, \widehat{p}) e^{-\beta \mathscr{H}(\widehat{q}, \widehat{p})}}{\int d \widehat{p} \int d \widehat{q} e^{-\beta \mathscr{H}(\widehat{q}, \widehat{p})}}
$$

When evaluating a MD simulation of the reduced system (4.47), our ability to calculate thermodynamic quantities is limited to the evaluation of averages and correlations of resolved phase variables under the RCD. The properties of the full system that we are interested in must be related to these quantities. The following two closely-related theorems will be useful:

Theorem 4.2 If $A$ is a resolved phase variable, then $\langle A\rangle_{R C}=\langle A\rangle_{C}$.

\section{Proof:}

$$
\begin{aligned}
\langle A\rangle_{R C} & =\frac{\int d \widehat{p} \int d \widehat{q} A(\widehat{q}, \widehat{p}) e^{-\beta \mathscr{H}(\widehat{q}, \widehat{p})}}{\int d \widehat{p} \int d \widehat{q} e^{-\beta \mathscr{H}(\widehat{q}, \widehat{p})}} \\
& =\frac{\frac{1}{c} \int d \widehat{p} \int d \widehat{q} A(\widehat{q}, \widehat{p}) \int d \widetilde{p} \int d \widetilde{q} e^{-\beta H(q, p)}}{\frac{1}{c} \int d \widehat{p} \int d \widehat{q} \int d \widetilde{p} \int d \widetilde{q} e^{-\beta H(q, p)}} \quad \text { by Hald's Theorem } \\
& =\frac{\int d p \int d q A(\widehat{q}, \widehat{p}) e^{-\beta H(q, p)}}{\int d p \int d q e^{-\beta H(q, p)}}=\langle A\rangle_{C}
\end{aligned}
$$

Theorem 4.3 Let $g^{(m)}\left(\widehat{q}_{1} \ldots \widehat{q}_{m}, \rho, T\right)$ be the m-particle distribution function derived from the potential $\phi$ of the full system, and let $\widehat{g}^{(m)}\left(\widehat{q}_{1} \ldots \widehat{q}_{m}, \widehat{\rho}, T\right)$ be the corresponding $m$-particle distribution function derived from the renormalized potential $\widehat{\phi}$. Then, for fixed $m$, $g^{(m)}\left(\widehat{q}_{1} \ldots \widehat{q}_{m}, \rho, T\right)=\widehat{g}^{(m)}\left(\widehat{q}_{1} \ldots \widehat{q}_{m}, \widehat{\rho}, T\right)$ in the thermodynamic limit.

Proof: From the definition of the m-particle distribution function [23], we have:

$$
\begin{aligned}
g^{(m)}\left(\widehat{q}_{1} \ldots \widehat{q}_{m}, \rho, T\right) & \equiv \frac{1}{\rho^{m}} \frac{N !}{(N-m) !} \frac{\int d \widehat{q}_{m+1} \ldots d \widehat{q}_{M} \int d \widetilde{q} e^{-\beta \phi(q)}}{\int d \widehat{q} \int d \widetilde{q} e^{-\beta \phi(q)}} \\
& =\frac{1}{\rho^{m}} \frac{N !}{(N-m) !} \frac{\int d \widehat{q}_{m+1} \ldots d \widehat{q}_{M} e^{-\beta \widehat{\phi}(\widehat{q})}}{\int d \widehat{q} e^{-\beta \widehat{\phi}(\widehat{q})}}
\end{aligned}
$$

\footnotetext{
${ }^{3}$ For a proof that this must occur for the Nosé-Hoover thermostat, see [47] or [26].
} 
Using the fact that $\rho=(N / M) \widehat{\rho}$, the prefactor can be rearranged as

$$
\begin{aligned}
\frac{1}{\rho^{m}} \frac{N !}{(N-m) !} & =\frac{1}{\widehat{\rho}^{m}} \frac{M^{m}}{N^{m}} \frac{N !}{(N-m) !} \\
& =\frac{1}{\widehat{\rho}^{m}} \frac{M !}{(M-m) !}\left\{\frac{(M-m) !}{M !} \frac{M^{m}}{N^{m}} \frac{N !}{(N-m) !}\right\} .
\end{aligned}
$$

In the thermodynamic limit, $N$ and $M$ become large, and the quantity in brackets can be simplified using Stirling's approximation:

$$
\begin{aligned}
\frac{(M-m) !}{M !} \frac{M^{m}}{N^{m}} \frac{N !}{(N-m) !} & \approx \frac{(M-m)^{M-m+\frac{1}{2}} e^{m-M}}{M^{M+\frac{1}{2}} e^{-M}} \frac{M^{m}}{N^{m}} \frac{N^{N+\frac{1}{2}} e^{-N}}{(N-m)^{N-m+\frac{1}{2}} e^{m-N}} \\
& =\frac{\left(1-\frac{m}{M}\right)^{M-m+\frac{1}{2}}}{\left(1-\frac{m}{N}\right)^{N-m+\frac{1}{2}}} \longrightarrow \frac{e^{-m}}{e^{-m}}=1,
\end{aligned}
$$

and the original expression becomes

$$
g^{(m)}\left(\widehat{q}_{1} \ldots \widehat{q}_{m}, \rho, T\right)=\frac{1}{\widehat{\rho}^{m}} \frac{M !}{(M-m) !} \frac{\int d \widehat{q}_{m+1} \ldots d \widehat{q}_{M} e^{-\beta \widehat{\phi}(\widehat{q})}}{\int d \widehat{q} e^{-\beta \widehat{\phi}(\widehat{q})}}=\widehat{g}^{(m)}\left(\widehat{q}_{1} \ldots \widehat{q}_{m}, \widehat{\rho}, T\right) .
$$

\subsubsection{Static Averages}

\section{Energy}

According to (3.9), the energy of the full system in 3 dimensions is given by

$$
E=\frac{3}{2} N k_{B} T+\langle\phi\rangle_{C},
$$

where the canonical average of the potential $\phi$ is the quantity typically evaluated in a simulation. We may express $\langle\phi\rangle_{C}$ in terms of the RDF of the full system as follows:

$$
\begin{aligned}
\langle\phi\rangle_{C} & =\frac{\int d q \sum_{i<j}^{M} U_{i j} e^{-\beta \phi(q)}}{\int d q e^{-\beta \phi(q)}} \\
& =\frac{N(N-1)}{2} \frac{\int d q U_{12} e^{-\beta \phi(q)}}{\int d q e^{-\beta \phi(q)}} \quad \text { because all particle pairs are equivalent. } \\
& =\frac{N(N-1)}{2} \int d q_{1} \int d q_{2} U_{12} \underbrace{\left.\frac{\int d q_{3} \ldots d q_{N} e^{-\beta \phi(q)}}{\int d q e^{-\beta \phi(q)}}\right\}}_{=\frac{(N-2) !}{N !} \rho^{2} g^{(2)}\left(\left|q_{12}\right|\right)} \\
& =\frac{\rho^{2}}{2} \int d q_{1} \int d q_{2} U\left(\left|q_{12}\right|\right) g^{(2)} \\
& =N \frac{\rho}{2} \int d r U(|r|) g^{(2)}(|r|) .
\end{aligned}
$$


Theorem 4.3 tells us that $g^{(2)}=\widehat{g}^{(2)}$, which allows us to write

$$
\begin{aligned}
\langle\phi\rangle_{C} & =N \frac{\rho}{2} \int d r U(|r|) \widehat{g}^{(2)}(|r|) \\
& =\left(\frac{N}{M}\right)^{2} M \frac{\widehat{\rho}}{2} \int d r U(|r|) \widehat{g}^{(2)}(|r|) \\
& =\left(\frac{N}{M}\right)^{2}\left\langle\phi_{r r}\right\rangle_{R C},
\end{aligned}
$$

where $\phi_{r r}$ is as defined in (4.8). This implies that the quantity

$$
\widehat{E}(M) \equiv \frac{3}{2} N k_{B} T+\left(\frac{N}{M}\right)^{2}\left\langle\phi_{r r}\right\rangle_{R C},
$$

if evaluated using the full expression (4.33) for $\widehat{\phi}$, should exactly reproduce the energy of the full system.

\section{Pressure}

An expression for the pressure similar to (4.51) can be derived in an analogous fashion. Equation (3.10) tells us that

$$
\begin{aligned}
P & =\rho k_{B} T-\frac{2}{3 V}\langle\mathcal{W}(q)\rangle_{C} \\
& =\rho k_{B} T-\frac{1}{3 V}\left\langle\sum_{i<j}^{N}\left|q_{i j}\right| U^{\prime}\left(\left|q_{i j}\right|\right)\right\rangle_{C},
\end{aligned}
$$

which can be expressed in terms of the RDF as

$$
P=\rho k_{B} T-\frac{\rho^{2}}{6} \int d r|r| U^{\prime}(|r|) g^{(2)}(|r|) .
$$

Using Theorem 4.3 to replace $g^{(2)}$ by $\widehat{g}^{(2)}$ and proceeding as before, we obtain

$$
\widehat{P}(M) \equiv \rho k_{B} T-\left(\frac{N}{M}\right)^{2} \frac{2}{3 V}\left\langle\mathcal{W}_{r r}(\widehat{q})\right\rangle_{R C},
$$

where

$$
\mathcal{W}_{r r}(\widehat{q}) \equiv \frac{1}{2} \sum_{i<j}^{M}\left|\widehat{q}_{i j}\right| U^{\prime}\left(\left|\widehat{q}_{i j}\right|\right)
$$

is the resolved virial. $\widehat{P}(M)$ should yield the exact pressure of the full system.

\section{Heat Capacity}

The heat capacity presents a somewhat more challenging problem than the energy or pressure. According to (3.11), the heat capacity can be written as

$$
C_{V}=\frac{3}{2} N k_{B}+\frac{1}{k_{B} T^{2}} \delta \phi^{2}
$$


where

$$
\delta \phi^{2}=\left\langle\phi^{2}\right\rangle_{C}-\langle\phi\rangle_{C}^{2}
$$

is the fluctuation in the potential energy, and is usually the quantity calculated in a simulation. The first term above can be broken up as follows:

$$
\begin{aligned}
\left\langle\phi^{2}\right\rangle_{C} & =\left\langle\sum_{i<j}^{N} \sum_{k<\ell}^{N} U_{i j} U_{k \ell}\right\rangle_{C} \\
& =\left\langle\begin{array}{c}
\text { terms with both } \\
\text { indices in common }
\end{array}\right\rangle_{C}+\left\langle\begin{array}{c}
\text { terms with one } \\
\text { index in common }
\end{array}\right\rangle_{C}+\left\langle\begin{array}{c}
\text { terms with no } \\
\text { indices in common }
\end{array}\right\rangle_{C} \\
& =\left\langle\sum_{i<j}^{N}{U_{i j}^{2}}^{2}\right\rangle_{C}+\left\langle 2 \sum_{i=1}^{N} \sum_{\substack{j<k \\
j, k \neq i}}^{N} U_{i j} U_{i k}\right\rangle_{C}+\left\langle\sum_{i<j}^{N} \sum_{\substack{k<\ell \\
k, \ell \neq i, j}}^{N} U_{i j} U_{k \ell}\right\rangle_{C}
\end{aligned}
$$

In each of the three quantities above, all terms of the sum yield the same contribution to the average, since all particles are equivalent. The first summation has $N(N-1) / 2$ terms, the second has $N(N-1)(N-2) / 2$ terms, and the third has $N(N-1)(N-2)(N-3) / 4$ terms. (4.54) can therefore be written as

$$
\begin{aligned}
\left\langle\phi^{2}\right\rangle_{C}=\frac{1}{2} N(N-1)\left\langle U_{12}^{2}\right\rangle_{C}+N(N-1)(N & -2)\left\langle U_{12} U_{13}\right\rangle_{C} \\
& +\frac{1}{4} N(N-1)(N-2)(N-3)\left\langle U_{12} U_{34}\right\rangle_{C} .
\end{aligned}
$$

Finally, using (4.50), Theorem 4.2, and the indistinguishability of the resolved particles, we obtain

$$
\begin{aligned}
& \delta \phi^{2}=\frac{N(N-1)}{M(M-1)}\left\langle\sum_{i<j}^{M} U_{i j}^{2}\right\rangle_{R C}+\frac{N(N-1)(N-2)}{M(M-1)(M-2)}\left\langle 2 \sum_{i=1}^{M} \sum_{\substack{j<k \\
j, k \neq i}}^{M} U_{i j} U_{i k}\right\rangle_{R C} \\
& +\frac{N(N-1)(N-2)(N-3)}{M(M-1)(M-2)(M-3)}\left\langle\sum_{i<j}^{M} \sum_{\substack{k<\ell \\
k, \ell \neq i, j}}^{M} U_{i j} U_{k \ell}\right\rangle_{R C}-\left(\frac{N(N-1)}{M(M-1)}\left\langle\phi_{r r}\right\rangle_{R C}\right)^{2} .
\end{aligned}
$$

We see that the $\delta \phi^{2}$ calculated under the RCD can be scaled to recover the same quantity calculated under the full distribution. However, unlike the corresponding expressions for energy and pressure, different pieces of $\delta \phi^{2}$ are scaled by different functions of $N$ and $M$. Note that we have used a different method to arrive at (4.55) than we used to obtain the expressions for energy and pressure. The reasons for this are subtle. Had we used distribution functions as before, we would have arrived at an expression similar to (4.55), except that all rational polynomials in $N$ and $M$ would be replaced by their large $N, M$ limit, i.e. $(N / M)^{2}$ instead of $N(N-1) / M(M-1)$, for example. This is a result of the assumption, inherent in the use of Theorem 4.3, that we are operating in the thermodynamic limit. In most cases treated here, this is a sound assumption. However, here this gives the wrong result for any values of $N$ and $M$, due to the fact that the accuracy of (4.55) depends 
on a significant degree of cancellation between the last two terms. This cancellation cannot be captured without using the exact form for the $N$ and $M$-dependent coefficients.

If we define

$$
\alpha_{1} \equiv \sum_{i<j}^{M} U_{i j}{ }^{2} \quad \alpha_{2} \equiv 2 \sum_{i=1}^{M} \sum_{\substack{j<k \\ j, k \neq i}}^{M} U_{i j} U_{i k} \quad \alpha_{3} \equiv \sum_{i<j}^{M} \sum_{\substack{k<\ell \\ k, \ell \neq i, j}}^{M} U_{i j} U_{k \ell},
$$

then (4.55) can be written as

$$
\begin{aligned}
\delta \phi^{2}=\frac{N(N-1)}{M(M-1)}\left\langle\alpha_{1}\right\rangle_{R C}+\frac{N(N-1)(N-2)}{M(M-1)(M-2)}\left\langle\alpha_{2}\right\rangle_{R C} & \\
& +\frac{N(N-1)(N-2)(N-3)}{M(M-1)(M-2)(M-3)}\left\langle\alpha_{3}\right\rangle_{R C}-\left(\frac{N(N-1)}{M(M-1)}\left\langle\phi_{r r}\right\rangle_{R C}\right)^{2} .
\end{aligned}
$$

Each of the $\alpha_{i}$ must be evaluated separately in order to implement (4.56). The most straightforward way to do this would be to keep track of the contribution to the total potential energy from each of the $M(M-1) / 2$ pairs of resolved particles. The products of these contributions could then be sorted into $\alpha_{1}, \alpha_{2}$, and $\alpha_{3}$ depending on the number of distinct indices. Unfortunately, the computational overhead associated with performing this sorting procedure at each timestep is at least $O\left(M^{2}\right)$, and is therefore untenable.

We can calculate the $\alpha_{i}$ via a more indirect route by defining the following two quantities:

$$
\phi_{i} \equiv \sum_{j \neq i}^{M} U_{i j} \quad \beta_{i} \equiv \sum_{j \neq i}^{M} U_{i j}^{2}
$$

By a simple process of rearranging indices, it is then possible to show that

$$
\begin{aligned}
\phi_{r r} & =\frac{1}{2} \sum_{i=1}^{M} \phi_{i} & \alpha_{1} & =\frac{1}{2} \sum_{i=1}^{M} \beta_{i} \\
\alpha_{2} & =\sum_{i=1}^{M}\left(\phi_{i}^{2}-\beta_{i}\right) & \alpha_{3} & =\phi_{r r}^{2}-\alpha_{1}-\alpha_{2}
\end{aligned}
$$

During a simulation, the $\phi_{i}$ and $\beta_{i}$ can be evaluated at each timestep in the course of calculating the interparticle forces with very little additional computational overhead. The calculation of the $\alpha_{i}$ then introduces an extra $O(M)$ process, which is acceptable.

Combining equations (4.53) and (4.56) shows that the quantity

$$
\begin{aligned}
\widehat{C}_{V}(M)=\frac{3}{2} N k_{B} & +\frac{1}{k_{B} T^{2}}\left[\frac{N(N-1)}{M(M-1)}\left\langle\alpha_{1}\right\rangle_{R C}+\frac{N(N-1)(N-2)}{M(M-1)(M-2)}\left\langle\alpha_{2}\right\rangle_{R C}\right. \\
& \left.+\frac{N(N-1)(N-2)(N-3)}{M(M-1)(M-2)(M-3)}\left\langle\alpha_{3}\right\rangle_{R C}-\left(\frac{N(N-1)}{M(M-1)}\left\langle\phi_{r r}\right\rangle_{R C}\right)^{2}\right]
\end{aligned}
$$

should reproduce the heat capacity of the full system exactly. 


\subsubsection{Equilibrium Autocorrelation Functions}

As we have seen, it is relatively simple to determine how static quantities, such as energy or pressure, must be scaled in order to reproduce the properties of the full system from data generated by the reduced dynamics (4.47). Equilibrium autocorrelation functions such as those described in Section 3.3, on the other hand, are extremely difficult to study analytically for $t>0$. In fact, the only factor we can directly control via scaling is the value of the autocorrelation function at $t=0$.

Consider some dynamical variable $A(q, p)$, which depends on both the resolved and unresolved variables. Its autocorrelation function, calculated in the context of the full system, is $C_{A A}(t)=\langle A(0) A(t)\rangle_{C}$. We define $\widehat{A}(\widehat{q}, \widehat{p})$ to be the corresponding quantity in the reduced system, with autocorrelation function $C_{\widehat{A} \widehat{A}}(t)=\langle\widehat{A}(0) \widehat{A}(t)\rangle_{R C}$. Our goal is to choose the scaling of $\widehat{A}$ to ensure that $C_{\widehat{A} \widehat{A}}(0)=C_{A A}(0)$. How these two autocorrelation functions compare for $t>0$ is beyond our control.

\section{Velocity Autocorrelation Function}

Let us consider the velocity autocorrelation function first. Under the full system, we have

$$
\begin{aligned}
C_{v v}(0) & =\frac{1}{3}\left\langle v_{i}(0) \cdot v_{i}(0)\right\rangle_{C} \quad & \text { for any } 1 \leq i \leq N \\
& =\frac{k_{B} T}{m}, &
\end{aligned}
$$

and for the reduced system

$$
\begin{aligned}
C_{\widehat{v} \widehat{v}}(0) & =\frac{1}{3}\left\langle\widehat{v}_{i}(0) \cdot \widehat{v}_{i}(0)\right\rangle_{R C} \quad & \text { for any } 1 \leq i \leq M \\
& =\frac{k_{B} T}{m} . &
\end{aligned}
$$

(Here $v_{i}=p_{i} / m$ and $\widehat{v}_{i}=\widehat{p}_{i} / m$.) Since both the full system and the reduced system are thermostated to maintain the same temperature $T$, no special scaling is required to ensure that the two velocity autocorrelation functions coincide at $t=0$.

\section{Shear Stress Autocorrelation Function}

The shear stress autocorrelation function $C_{\Xi \Xi}(t)=\left\langle\Xi_{\mu \nu}(0) \Xi_{\mu \nu}(t)\right\rangle_{C}$ is a much more difficult and interesting case. According to equation (3.14), for the full system

$$
\Xi_{\mu \nu}=\frac{1}{V} \sum_{i=1}^{N}\left(\frac{1}{m} p_{i, \mu} p_{i, \nu}-q_{i, \mu} \frac{\partial}{\partial q_{i, \nu}} \phi(q)\right),
$$

in which case it can be shown (see Appendix A) that

$$
C_{\Xi \Xi}(0)=\frac{k_{B} T}{V}\left\{\rho k_{B} T+\frac{2 \pi}{15} \rho^{2} \int_{0}^{\infty} d r \frac{d}{d r}\left(r^{4} U^{\prime}(r)\right) g^{(2)}(r)\right\} .
$$

For the reduced system, we define

$$
\widehat{\Xi}_{\mu \nu}=\frac{1}{V} \sum_{i=1}^{M}\left(a \frac{1}{m} \widehat{p}_{i, \mu} \widehat{p}_{i, \nu}-b \widehat{q}_{i, \mu} \frac{\partial}{\partial \widehat{q}_{i, \nu}} \phi_{r r}(\widehat{q})\right),
$$


where $a$ and $b$ are scaling constants to be determined. A derivation identical to that which generated (4.58) yields

$$
C_{\widehat{\Xi} \widehat{\Xi}}(0)=\frac{k_{B} T}{V}\left\{a^{2} \widehat{\rho} k_{B} T+\frac{2 \pi}{15} b^{2} \widehat{\rho}^{2} \int_{0}^{\infty} d r \frac{d}{d r}\left(r^{4} U^{\prime}(r)\right) \widehat{g}^{(2)}(r)\right\} .
$$

If we now apply Theorem 4.3, and use the fact that $\widehat{\rho}=(M / N) \rho$, we arrive at

$$
C_{\widehat{\Xi} \widehat{\Xi}}(0)=a^{2} \frac{M}{N} \rho k_{B} T+\frac{2 \pi}{15} b^{2}\left(\frac{M}{N}\right)^{2} \rho^{2} \int_{0}^{\infty} d r \frac{d}{d r}\left(r^{4} U^{\prime}(r)\right) g^{(2)}(r),
$$

which, when compared with (4.58), implies that

$$
a=\left(\frac{N}{M}\right)^{\frac{1}{2}} \quad b=\frac{N}{M} .
$$

Thus, the autocorrelation function of the resolved quantity

$$
\widehat{\Xi}_{\mu \nu}=\frac{1}{V} \sum_{i=1}^{M}\left(\left(\frac{N}{M}\right)^{\frac{1}{2}} \frac{1}{m} \widehat{p}_{i, \mu} \widehat{p}_{i, \nu}-\frac{N}{M} \widehat{q}_{i, \mu} \frac{\partial}{\partial \widehat{q}_{i, \nu}} \phi_{r r}(\widehat{q})\right)
$$

will be guaranteed to coincide with $C_{\Xi \Xi}(t)$ at $t=0$, i.e.

$$
\left\langle\widehat{\Xi}_{\mu \nu}^{2}(0)\right\rangle_{R C}=\left\langle\Xi_{\mu \nu}^{2}(0)\right\rangle_{C} .
$$

Keep in mind, however, that our approximation (4.48) to the renormalized potential will prevent equation (4.60) from being obeyed exactly at moderate to high densities.

There is no reason to suspect that the above scaling procedure, which constrains the values of the autocorrelation functions at $t=0$, will reproduce these functions for the full system at $t>0$. In fact, we will see that FOOP is not nearly as effective in reproducing the velocity and shear stress autocorrelation functions as it is for calculating static quantities. This is to be expected. Autocorrelation functions embody the correlations of dynamical quantities over extended periods of time, and are thus closely dependent upon the memory and forcing terms that we ignored when deriving FOOP.

\subsection{Consequences of Neglecting Many-Particle Interactions}

In an actual reduced simulation, in which expressions such as (4.51), (4.52), and (4.57) are used to determine the properties of the full system, the accuracy of the results will depend on the degree to which the pair approximation (4.46) is valid. When many-particles effects play a significant role in the dynamics, we expect these expressions to yield values that differ appreciably from the true energy, pressure, and heat capacity.

Consider a system of interacting particles with a typical Hamiltonian of the form $H=K+\phi$, where $K$ is the kinetic energy and $\phi$ is the potential energy. It is common for the phase of such a system to be classified according to the relative magnitudes of $\langle K\rangle$ and $\langle\phi\rangle:|\langle K\rangle| \gg|\langle\phi\rangle|$ corresponds to a gaseous phase, $|\langle K\rangle| \ll|\langle\phi\rangle|$ indicates a solid, and $|\langle K\rangle| \sim|\langle\phi\rangle|$ is usually taken to correspond to a liquid.

The same type of reasoning can be used to gain some qualitative insight into when (4.46) should be expected to fail. As (4.29) indicates, the renormalized potential $\widehat{\phi}$ may be written 
as a sum of a two-particle term, a three-particle term, etc. The $n$-particle contribution to $\widehat{\phi}$ can be expressed as

$$
\widehat{\phi}^{(n)}(\widehat{q})=\sum_{i_{1}<\ldots<i_{n}}^{M} \widehat{U}_{n}\left(\widehat{q}_{i_{1}}, \ldots, \widehat{q}_{i_{n}}\right),
$$

where the form of $\widehat{U}_{n}$ depends on $\widetilde{\rho}$ and $T$. Note that $\widehat{U}_{2}=\widehat{U}$, the renormalized pair potential introduced at the end of Section 4.2.1. The average of $\widehat{\phi}$ in the (exact) reduced system is

$$
\langle\widehat{\phi}\rangle_{R C}=\left\langle\widehat{\phi}^{(2)}\right\rangle_{R C}+\left\langle\widehat{\phi}^{(3)}\right\rangle_{R C}+\left\langle\widehat{\phi}^{(4)}\right\rangle_{R C}+\ldots
$$

Note that each of these terms must be an extensive quantity, i.e. proportional to the number of resolved particles $M$, in order for the average energy per particle to be stable in the thermodynamic limit. Understanding when the various terms in (4.61) are large or small will take us a long way towards determining the appropriateness of (4.46), as well as towards explaining several of the numerical results to come later in this chapter.

We begin by defining some terminology. A set of $n$ resolved particles, with coordinates $\left(\widehat{q}_{1}, \ldots, \widehat{q}_{n}\right)$, is said to be "within range $R$ of each other" if $\left|\widehat{q}_{i}-\widehat{q}_{j}\right| \leq R$ for all $1 \leq i, j \leq n$. We define $R_{n}$ to be the "range" of $\widehat{U}_{n}$, in the sense that $\widehat{U}_{n}\left(\widehat{q}_{1}, \ldots, \widehat{q}_{n}\right)=0$ unless $\left(\widehat{q}_{1}, \ldots, \widehat{q}_{n}\right)$ are within range $R_{n}$ of each other. For a bare potential such as the Lennard-Jones potential, $\widehat{U}_{n}$ will never be strictly zero as long as its arguments are finite, so we take this definition to mean that $R_{n}$ is the range beyond which $\widehat{U}_{n}$ is approximately zero. For example, the renormalized pair potentials shown in Figure 4.2 have $R_{2}^{*} \sim 2$ or 3 . In general, $R_{n}$ will be a function of $\widetilde{\rho}$ and $T$.

For a reduced system containing $M$ resolved particles at a density of $\widehat{\rho}$, we define a quantity $\mathcal{N}_{n}(\widehat{\rho}, R, T)$ by requiring that $M \mathcal{N}_{n}(\widehat{\rho}, R, T)$ be the average number of $n$-tuplets of particles within range $R$ of each other in the system at equilibrium. Clearly, the maximum possible value of $M \mathcal{N}_{n}$ is $\left(\begin{array}{c}M \\ n\end{array}\right)$. However, the actual value in a typical system will be far less than this. As will be shown below, the fact that $\mathcal{N}_{n}$ is an intensive quantity follows from the fact that $\left\langle\widehat{\phi}^{(n)}\right\rangle$ is extensive.

Finally, we introduce $\bar{U}_{n}(\widetilde{\rho}, T)$, which is defined as the magnitude of the average value of $\widehat{U}_{n}\left(\widehat{q}_{1}, \ldots, \widehat{q}_{n}\right)$, given that the coordinates $\left(\widehat{q}_{1}, \ldots, \widehat{q}_{n}\right)$ are within range $R_{n}$ of each other. In other words, $\bar{U}_{n}$ is the size of a typical contribution to $\widehat{\phi}^{(n)}$ due to $n$ particles that are in the process of interacting via $\widehat{U}_{n}$.

Given the above definitions, a few remarks can be made concerning the behavior of $R_{n}$, $\mathcal{N}_{n}$, and $\bar{U}_{n}$. For simplicity, we will leave out the dependence of these three quantities on $T$ here, since this is an inherently constant-temperature problem.

- $R_{n}(\widetilde{\rho})$ is a monotonically increasing function of $\widetilde{\rho}$.

As $\widetilde{\rho}$ increases from 0 , the diagrammatic expansion of $\widehat{U}_{n}$ contains significant contributions from higher and higher order diagrams, which have larger ranges than lower order diagrams. This effect is evident for $n=2$ in Figures 4.2 and 4.4.

- $R_{n}(\widetilde{\rho}) \rightarrow R_{n}(0)>0$ as $\widetilde{\rho} \rightarrow 0$. For $n=2, \widehat{U}_{2} \rightarrow U$, which has a finite, nonzero range. For $n>2$, the shape of $\widehat{U}_{n}$ is dominated by the first diagram in its series, which likewise has a finite, nonzero range.

- $\mathcal{N}_{n}(\widehat{\rho}, R)$ is a monotonically increasing function of both $\widehat{\rho}$ and $R$. 
- $\mathcal{N}_{n}(\widehat{\rho}, R) \rightarrow 0$ as either $\widehat{\rho} \rightarrow 0$ or $R \rightarrow 0$.

As either the density of particles $\widehat{\rho}$ or the range $R$ decrease, the probability that $n$ particles will fall within range $R$ of each other decreases as well, and vice versa.

- As $\widetilde{\rho} \rightarrow 0, \bar{U}_{2}(\widetilde{\rho}) \rightarrow \bar{U}_{2}(0)>0$.

When $\widetilde{\rho}=0$, the renormalized pair potential reduces to the bare potential, which has nonzero magnitude.

- For $n>2, \bar{U}_{n}(\widetilde{\rho}) \rightarrow 0$ as $\widetilde{\rho} \rightarrow 0$.

This follows from the fact that $\widehat{U}_{n}$ for $n>2$ is linear in $\widetilde{\rho}$ to lowest order.

In addition, note that in a noninteracting, ideal gas, where the particle positions are uncorrelated and distributed uniformly throughout the domain, there is no inherent scale length of the system independent of the density, and $\mathcal{N}_{n}$ will satisfy

$$
\mathcal{N}_{n}(\widehat{\rho}, R)=\mathcal{N}_{n}^{\text {ideal }}\left(\widehat{\rho} R^{d}\right),
$$

where $d$ is the dimensionality of the system. Here $\mathcal{N}_{n}^{\text {ideal }}$ is a monotonically increasing function of its argument. Equation (4.62) follows from a simple scaling argument that involves considering the average number of particles within a ball of radius $R$, which is proportional to $\widehat{\rho} R^{d}$. For an interacting system, (4.62) fails, due to the fact that a natural, density-independent microscopic length scale exists in the form of $\sigma$, the range of the interparticle potential. However, equation (4.62) should still hold approximately when $R \gg \sigma$ or $\widehat{\rho}^{-1 / d} \gg \sigma$.

The magnitude of the $n^{\text {th }}$ order term in (4.61) can now be written as

$$
\begin{aligned}
\left|\left\langle\widehat{\phi}^{(n)}\right\rangle_{R C}\right| & =\left(\begin{array}{c}
\text { Average number of resolved } \\
n \text {-tuplets interacting via } \widehat{U}_{n}
\end{array}\right) \times\left|\left(\begin{array}{c}
\text { Average potential energy due } \\
\text { to } n \text { particles in the process } \\
\text { of interacting via } \widehat{U}_{n}
\end{array}\right)\right| \\
& =M \mathcal{N}_{n}\left(\widehat{\rho}, R_{n}(\widetilde{\rho})\right) \bar{U}_{n}(\widetilde{\rho}) .
\end{aligned}
$$

Note that since $\left\langle\widehat{\phi}^{(n)}\right\rangle_{R C}$ is necessarily extensive, and $\bar{U}_{n}$ is intensive by definition, this indicates that $\mathcal{N}_{n}$ is intensive. The fact that $\widehat{\rho}=\rho-\widetilde{\rho}$ allows us to rewrite (4.63) as

$$
\frac{1}{M}\left|\left\langle\widehat{\phi}^{(n)}\right\rangle_{R C}\right|=\mathcal{N}_{n}\left(\rho-\widetilde{\rho}, R_{n}(\widetilde{\rho})\right) \bar{U}_{n}(\widetilde{\rho}) .
$$

By definition, $\widetilde{\rho}$ is constrained to lie between 0 and $\rho$. Using the properties of $R_{n}, \mathcal{N}_{n}$, and $\bar{U}_{n}$ described above, we can study (4.64) in each of these two limiting cases.

For $\widetilde{\rho} \rightarrow 0$, we have

$$
\lim _{\widetilde{\rho} \rightarrow 0} \frac{1}{M}\left|\left\langle\widehat{\phi}^{(n)}\right\rangle_{R C}\right|=\underbrace{\mathcal{N}_{n}\left(\rho, R_{n}(0)\right)}_{>0} \underbrace{\bar{U}_{n}(0)}_{\substack{>0 \\
=0}}=\left\{\begin{array}{cc}
\mathcal{N}_{2}\left(\rho, R_{2}(0)\right) \bar{U}_{2}(0) & n=2 \\
0 & n>2
\end{array} .\right.
$$

On the other hand, when $\widetilde{\rho} \rightarrow \rho,(4.64)$ becomes

$$
\lim _{\widetilde{\rho} \rightarrow \rho} \frac{1}{M}\left|\left\langle\widehat{\phi}^{(n)}\right\rangle_{R C}\right|=\underbrace{\mathcal{N}_{n}\left(0, R_{n}(\rho)\right)}_{=0} \underbrace{\bar{U}_{n}(\rho)}_{>0}=0 .
$$


Equations (4.65) and (4.66) show that, for $n>2$, the error associated with ignoring the $n^{\text {th }}$ order term in (4.29) will drop to zero when either $\widetilde{\rho} \rightarrow 0$ or $\widetilde{\rho} \rightarrow \rho$. Stated in a more applicable way, for a reduced system of size $M$, the error associated with making the pair approximation (4.46) will drop to zero when either $M \rightarrow N$ or $M \rightarrow 0$. The first of these is unremarkable, since when $M=N$, we recover the exact full system (3.4). The latter, however, is surprising, and seems to imply that we can gain something for nothing by working with a reduced system containing only a handful of resolved particles. In reality, of course, a reduced system with such a small value of $M$ would be plagued by other sources of error. In particular, the errors in numerically calculating canonical averages, which typically scale as $M^{-1 / 2}$, become large in this limit. There would also be errors of order $M^{-1}$ associated with the fact that very small $M$ values place the system too far from the thermodynamic limit. It is an empirical rule of thumb in MD simulation that one must have at least several hundred particles in the system in order for finite system-size effects of this kind to be insignificant compared to other types of error (i.e. rounding error, finite timestep error, etc) [3]. Nevertheless, equations (4.65) and (4.66) make a definite prediction: That as the size $M$ of our reduced system decreases from $N$, the error due to (4.46) will first increase from zero, before attaining a maximum (or maxima) at some intermediate value, and then decreasing until $M$ becomes moderately small, at which point other sources of error become dominant.

All of what has been said so far in this section has been very qualitative. It would be convenient, however, to have some quantitative means of estimating the value of $M$ at which our pair approximation fails. Ideally, this would take the form of two functions, $\rho^{+}(\rho)$ and $\rho^{-}(\rho)$, such that (4.46) fails for $\tilde{\rho}$ in the range $\rho^{-}(\rho) \leq \widetilde{\rho} \leq \rho^{+}(\rho)$. For fixed $\rho, \rho^{+}$and $\rho^{-}$ could probably best be estimated by determining the values of $\widetilde{\rho}$ for which the magnitude of the triplet potential is comparable to that of the pair potential, i.e.

$$
\frac{1}{M}\left|\left\langle\widehat{\phi}^{(2)}\right\rangle_{R C}\right| \sim \frac{1}{M}\left|\left\langle\widehat{\phi}^{(3)}\right\rangle_{R C}\right|
$$

or equivalently, by (4.64),

$$
\mathcal{N}_{2}\left(\rho-\widetilde{\rho}, R_{2}(\widetilde{\rho})\right) \bar{U}_{2}(\widetilde{\rho}) \sim \mathcal{N}_{3}\left(\rho-\widetilde{\rho}, R_{3}(\widetilde{\rho})\right) \bar{U}_{3}(\widetilde{\rho}) .
$$

Unfortunately, the quantitative forms of the functions $R_{n}, \mathcal{N}_{n}$, and $\bar{U}_{n}$ are extremely difficult to determine either analytically or numerically, since they require knowledge of the exact form of the renormalized potential in order to be calculated. For this reason, the limits of our approximation will have to be found empirically by direct simulation of the reduced system.

\subsection{Results}

\subsubsection{Full System}

In order to serve as a baseline to which results from various reduced systems may later be compared, simulations of the full system (3.4) were done using the modified velocity Verlet scheme (3.22). The full system consisted of $N=4000$ Lennard-Jones particles with a cutoff radius of $r_{c}^{*}=2.5$. As described previously, the temperature was chosen to be $T^{*}=1.6$, which, according to (3.25), yields a timestep of $\Delta t^{*} \approx 0.005$. These simulations were performed for densities in the range $\rho^{*} \in[0.1,1.1]$, at intervals of $\Delta \rho^{*}=0.05$. In order to 


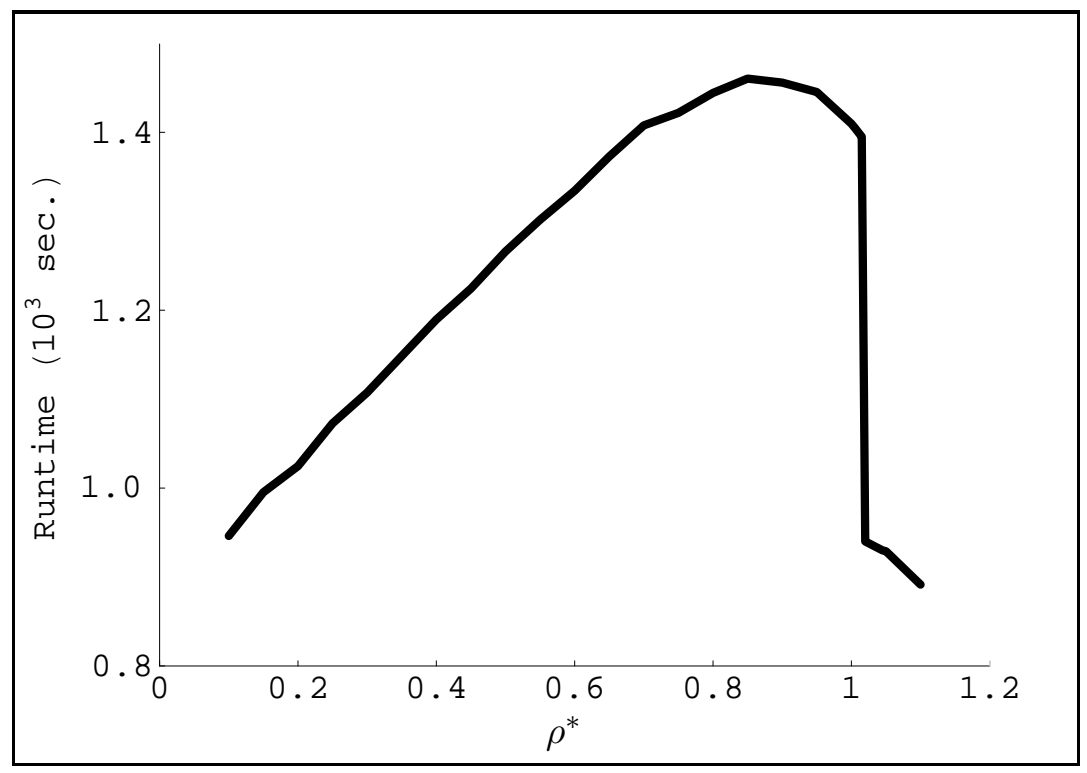

Figure 4.5: Runtime of the full system as a function of the reduced density $\rho^{*}$

check that the algorithm yields accurate results, additional simulations were performed for $\rho^{*} \in[0.955,1.045]$, at intervals of $\Delta \rho^{*}=0.005$. This range of densities contains the fluidsolid phase transition at this temperature, which, according to the Lennard-Jones phase diagram in Section 3.5, occurs at $\rho^{*} \approx 1.02$. Thermodynamic averages determined from these simulations, such as energy or pressure, should have a discontinuity at this density. Furthermore, we expect the heat capacity to diverge, due to the fact that energy added to a system at the fluid-solid transition will go into "melting" the solid, rather than increasing the kinetic temperature.

In each simulation, the system was initially integrated for 1000 timesteps, during which it was allowed to equilibrate, followed by 50,000 timesteps during which data was taken. It is interesting to look at the runtime of these simulations as a function of density, which is plotted in Figure 4.5. As the density increases from zero, the average number of neighbors associated with each particle grows. Thus a larger number of interparticle force calculations must be performed per timestep, and the runtime increases. At high densities, however, the freedom of individual particles to move around the domain is curtailed, and the neighbor list needs to be recalculated less frequently, which causes the runtime to decrease. In fact, at sufficiently high densities a point is reached at which particles may not move past one another to any extent, and are trapped in an effective potential well due to the combined interactions with their neighbors. Past this point, the neighbor list need only be calculated once at the beginning of the simulation, and, as can be seen in Figure 4.5, the runtime drops considerably. The density at which this occurs is the location of the fluid-solid phase transition. According to these numerics, the location of the transition falls in the interval $1.015 \leq \rho^{*} \leq 1.020$, in accord with our expectations.

The energy per particle $E / N$, the pressure $P$, and the heat capacity per particle $C_{V} / N$ of the full system were evaluated using equations (3.9), (3.10), and (3.11), respectively. The results of these calculations are shown as functions of density in Figures 4.6, 4.7, and 4.8. Note that, as expected, there are discontinuities in the energy and pressure, as well 


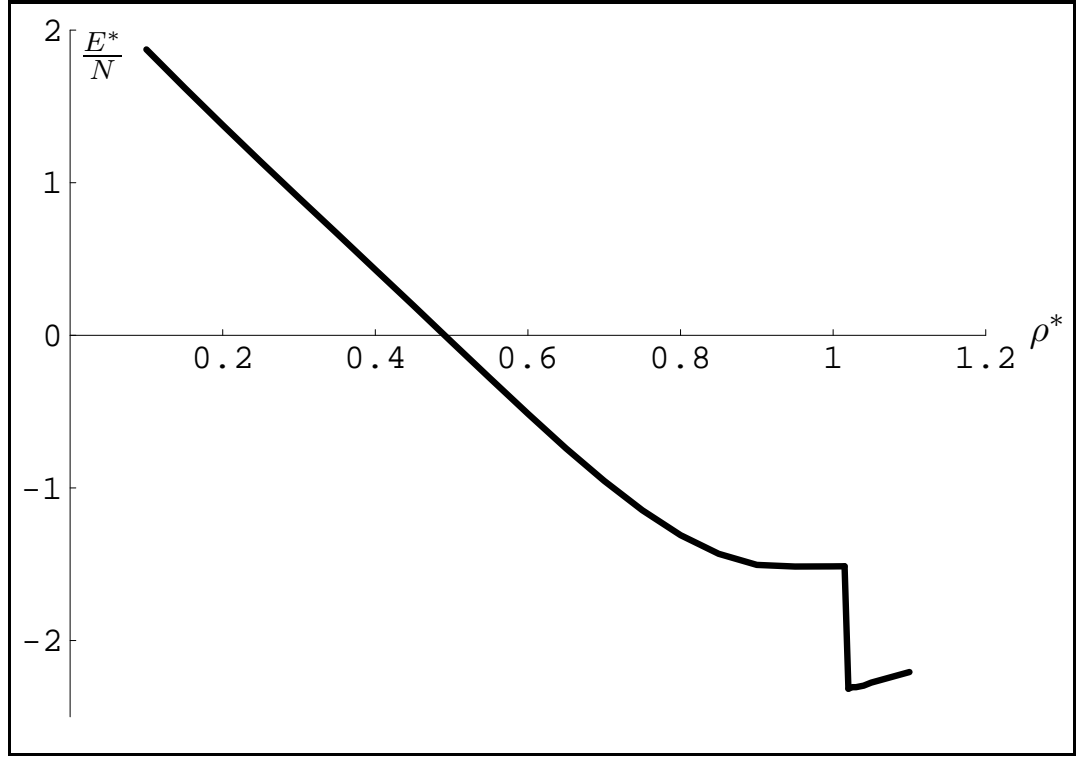

Figure 4.6: Energy per particle $E^{*} / N$ of the full system as a function of density, in reduced units

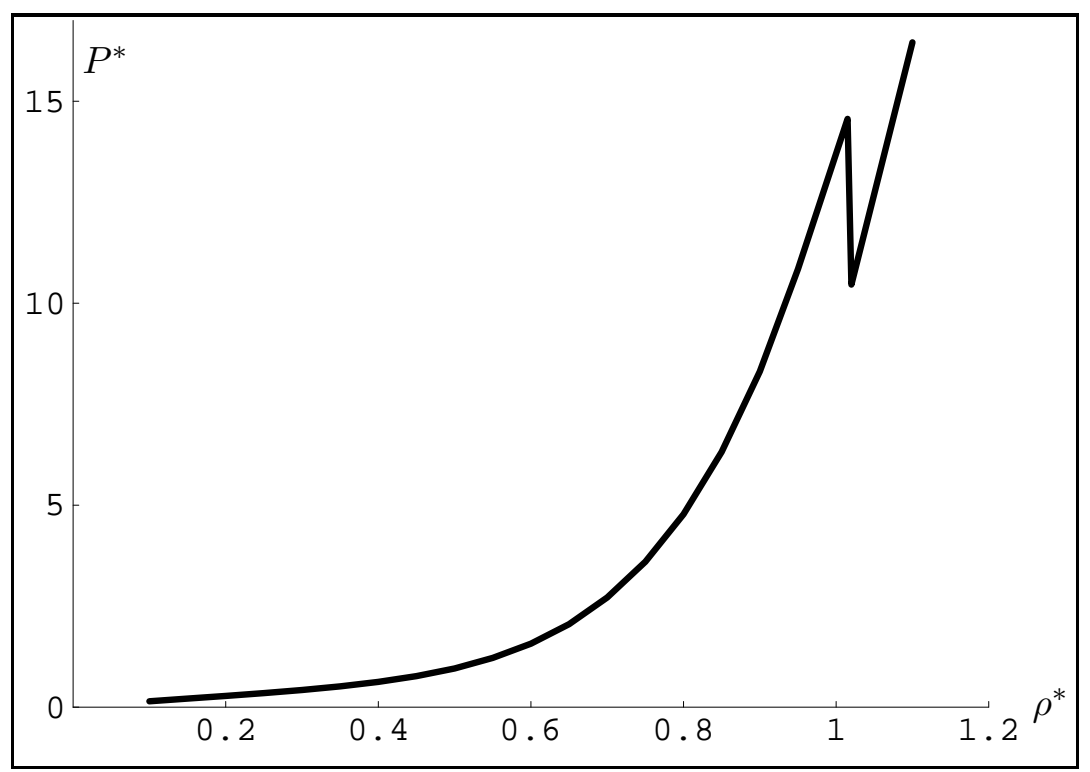

Figure 4.7: Pressure $P^{*}$ of the full system as a function of density, in reduced units 


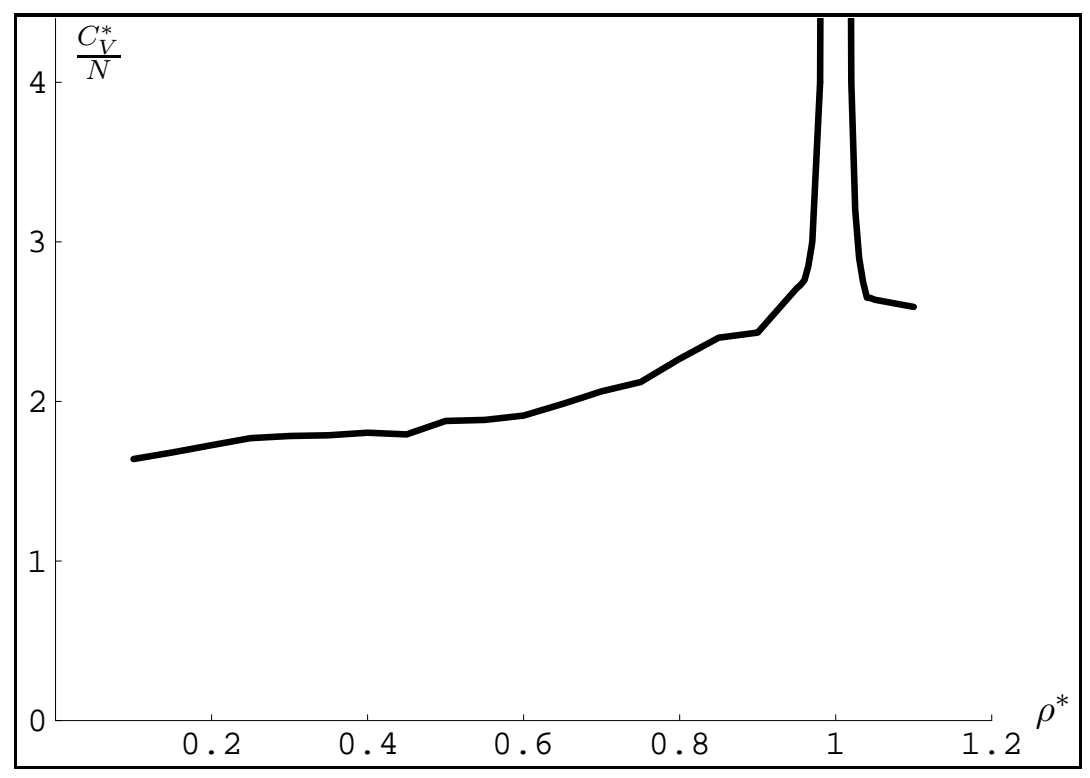

Figure 4.8: Heat capacity per particle $C_{V}^{*} / N$ of the full system as a function of density, in reduced units

as a divergence in the heat capacity, within the interval $1.015 \leq \rho^{*} \leq 1.020$ of the phase transition. Note also that the results for the heat capacity are somewhat noisier than those for either energy or pressure. This occurs universally in MD [3], and is a consequence of the fact that the heat capacity represents the mean fluctuation in a quantity, whereas energy and pressure are simple averages.

Velocity and shear stress autocorrelation functions were also calculated for the full system. These will be discussed below.

\subsubsection{Reduced Systems}

The pair-approximated FOOP system ((4.47) with (4.48)) and the Galerkin system (4.13) were each simulated using the same scheme (3.22) as the full system, as well as the same values of $T^{*}$ and $\Delta t^{*}$, and the same number of timesteps. For every density $\rho^{*} \in[0.1,1.1]$ at which the full system was studied, five different values of $\mathrm{M}$ were tried for each of the two reduced models: $M=2916,2048,1372,864$, and 500. (The reduced systems were not simulated at the closely-spaced densities in the range [0.955, 1.045], which were used solely to verify the reliability of our implementation of the algorithm (3.22) against established results.) As explained in Section 3.4.2, the reason for these particular values of $M$ is that the system is initialized in a face-centered cubic configuration, which constrains $M$ to be of the form $4 n_{c}^{3}$, where $n_{c}$ is the number of unit cells in each spatial direction. The density of resolved particles for each of these reduced simulations was $\widehat{\rho}^{*}=(M / N) \rho^{*}$. Furthermore, the unresolved density $\widetilde{\rho}^{*}=((N-M) / N) \rho^{*}$ was used for each FOOP system to calculate the form of the renormalized pair potential $\widehat{U}$, using the methods outlined in this chapter. For $\widetilde{\rho}^{*} \leq 0.25$, the diagrammatic sum truncated to $2^{\text {nd }}$ order was used, and for $\widetilde{\rho}^{*}>0.25$ the RDF calculated by MD was used to find $\widehat{U}$.

One complication that arises in dealing with the renormalized pair potential is that it 


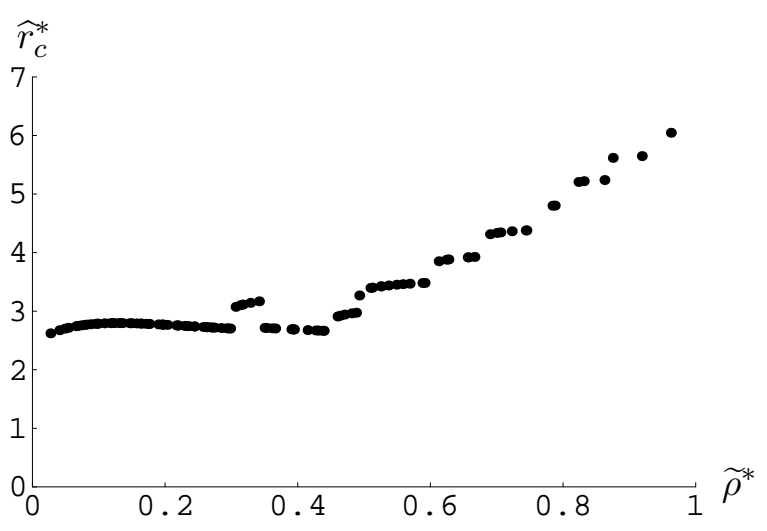

Figure 4.9: Cutoff radius $\widehat{r}_{c}$ of the renormalized pair potential as a function of the unresolved density $\widetilde{\rho}^{*}$.

has a longer range than the bare potential, as is evident in Figures 4.2 and 4.4. For fixed temperature, the unresolved density $\widetilde{\rho}$ alone determines its shape. In general, the larger $\widetilde{\rho}$ is, the longer the range of $\widehat{U}$. Therefore, a different cutoff radius $\widehat{r}_{c}$ was used for each distinct value of $\widetilde{\rho}$ studied by FOOP. This cutoff radius was determined in the following manner: if $r_{c}$ was the cutoff radius used in the full system $\left(r_{c}^{*}=2.5\right.$ in our case), then $\widehat{r}_{c}$ was chosen to be the smallest value of $r$ such that $\left|\widehat{U}\left(r^{\prime}\right)\right| \leq\left|U\left(r_{c}\right)\right|$ for all $r^{\prime} \geq r$. The cutoff radius of the FOOP system, as determined by this method, is shown in Figure 4.9 for each value of $\widetilde{\rho}^{*}$ to be examined. As expected, the cutoff radius is generally larger for larger $\widetilde{\rho}^{*}$. The apparent discontinuities in the graph of $\widehat{r}_{c}^{*}$ as a function of $\widetilde{\rho}^{*}$ are a result of maxima and minima of the renormalized pair potential that rise to cross the $|\widehat{U}|=\left|U\left(r_{c}\right)\right|$ threshold as $\widetilde{\rho}^{*}$ increases.

The fact that a larger cutoff radius must be used for larger values of $\widetilde{\rho}$ has significant consequences for the running speed of FOOP simulations. When $M$ is small or $\rho$ is large, $\widehat{r}_{c}$ increases, and the neighbor list of each particle grows in length. With a greater number of interparticle forces to evaluate per timestep, the FOOP system requires more time to simulate than the corresponding Galerkin system.

The "relative runtime" is defined as

$$
\text { Relative Runtime } \equiv \frac{(\text { Runtime of the reduced system })}{\left(\begin{array}{c}
\text { Runtime of the full system at } \\
\text { the same density and temperature }
\end{array}\right)}
$$

Thus, relative runtimes less than 1 indicate that the reduced system runs faster than the full system, whereas values greater than 1 mean that it is slower. Figures 4.10 and 4.11 show, for each value of $M$, the relative runtimes of the FOOP systems and the Galerkin systems, respectively, as functions of density. Here, and throughout this thesis, data obtained by simulating the reduced models will be graphed as a function of $\rho^{*}$, the density of the full system they seek to mimic, rather than as a function of $\widehat{\rho}^{*}$, the actual density of resolved particles. This allows easy comparison between the full system and reduced system results. 


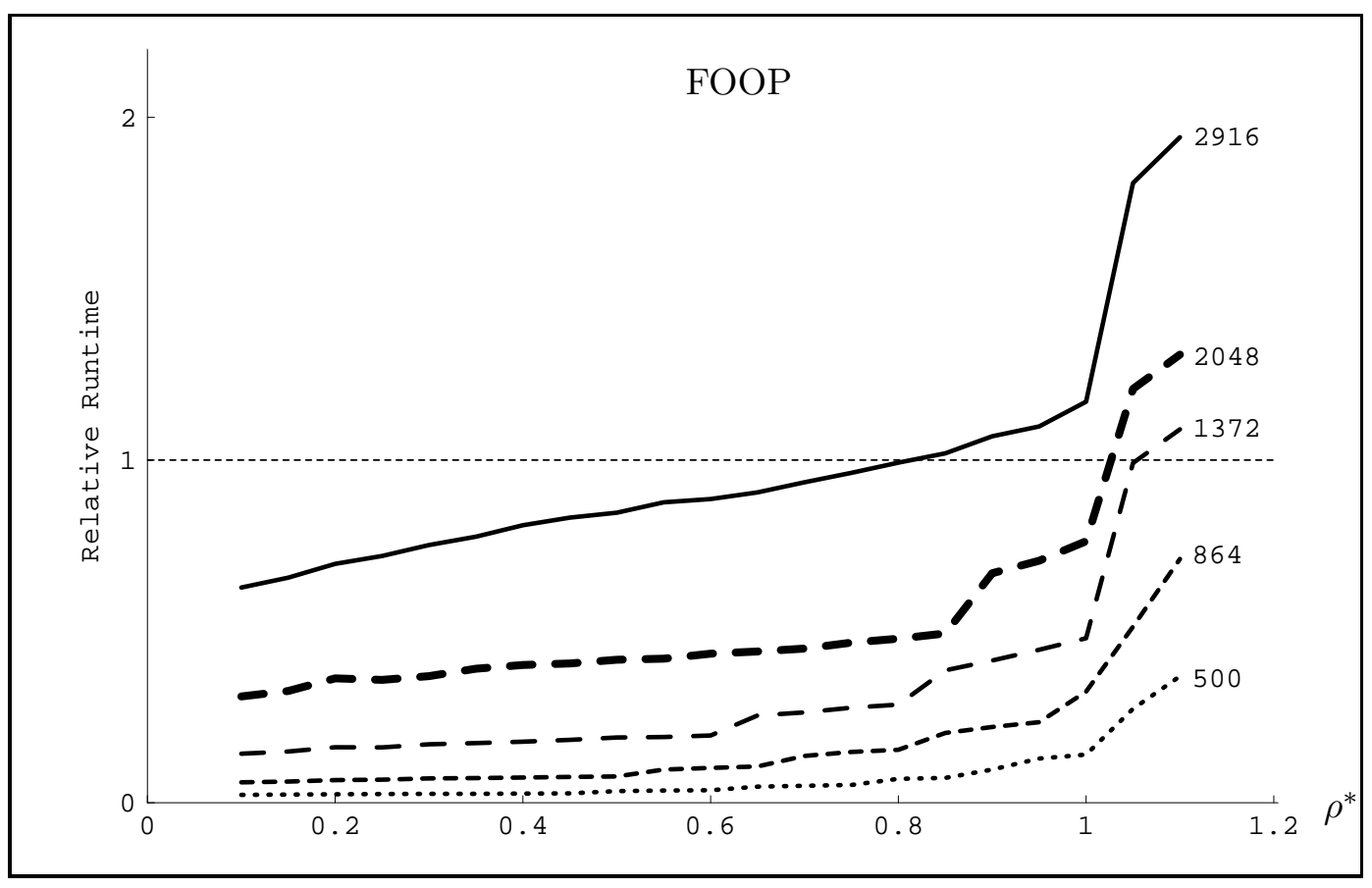

Figure 4.10: Relative runtimes of the FOOP systems as functions of reduced density, and for various values of $M$

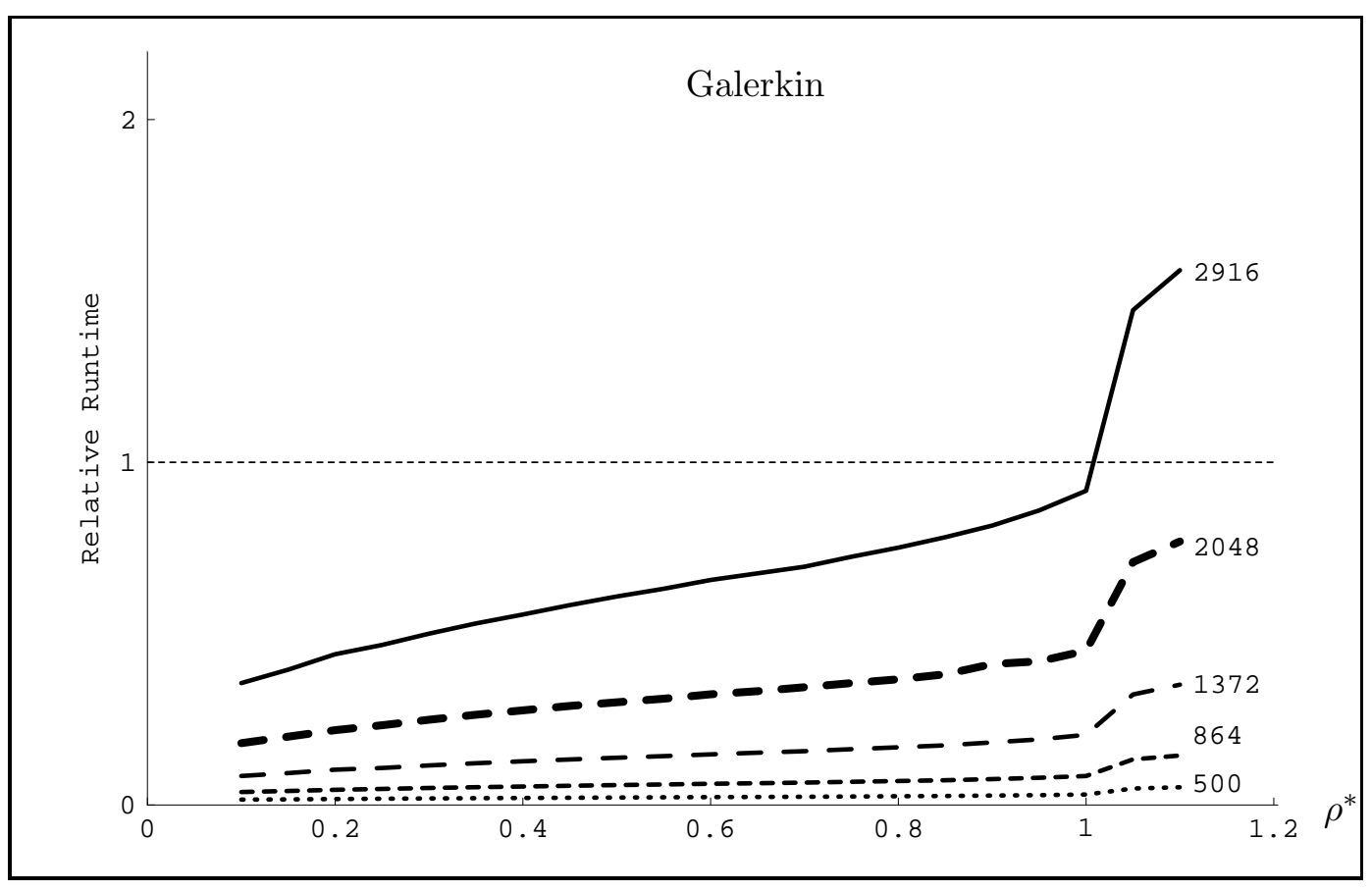

Figure 4.11: Relative runtimes of the Galerkin systems as functions of reduced density, and for various values of $M$ 
As should be expected, the smaller the value of $M$, the faster the reduced systems run. Furthermore, for fixed $M$ and $\rho^{*}$, the Galerkin system always runs faster than the FOOP system. This is a result of the fact that the renormalized potential has a larger cutoff radius, as explained above. It may seem peculiar at first that at high densities the Galerkin system with $M=2916$ requires more time to run than the full system. In fact, the increase in the relative runtime of all of the reduced systems for $\rho^{*} \gtrsim 1$ is simply a consequence of the full system's phase transition, which causes it to run faster. This implies that in order to approximate the solid phase via FOOP, one must use a sufficiently small value of $M$. Otherwise, little will be gained in terms of computational advantage.

\section{Static Averages}

The scaled energy per particle $\widehat{E}^{*}(M) / N$, the scaled pressure $\widehat{P}^{*}(M)$, and the scaled heat capacity per particle $\widehat{C}_{V}^{*}(M) / N$ were calculated during each of the reduced simulations, using (4.51), (4.52), and (4.57), respectively. The FOOP energy, pressure, and heat capacity are shown in Figures 4.12, 4.14, and 4.16, respectively, along with the same result from the full system for comparison. The same three quantities calculated via the Galerkin system are shown in Figures 4.13, 4.15, and 4.17.

We will discuss the energy and pressure results first. We see that, for both the energy and the pressure, FOOP performs significantly better than the Galerkin method. The energy and pressure curves derived from FOOP coincide nearly perfectly with the exact results from the full system, up to $\rho^{*} \approx 0.7$ and $\rho^{*} \approx 0.5$, respectively. By contrast, the Galerkin results begin to diverge from the exact curves both at a lower density and to a greater degree. This is as expected, since the Galerkin method makes no attempt to account for the influence of the unresolved particles.

It is important to note that FOOP fails to capture the behavior of the energy and pressure in the vicinity of the phase transition at $\rho^{*} \approx 1$. The reason for this is that the fluid-solid transition is, by definition, entirely dependent upon the many-particle effects that we have neglected. In a way, a solid comprised of $N$ molecules can be thought of as a single $N$-tuplet of particles interacting via a long-range $N$-particle potential. Clearly, no reduced system that includes only pairwise interactions can adequately model a solid or dense liquid. However, if we could somehow calculate $\widehat{\phi}$ exactly, the curves from the full system and the curves for each of the FOOP systems would necessarily lie on top of one another, to within an error of order $1 / \sqrt{M n_{s}}$ resulting from finite system sizes and run lengths, where $n_{s}$ is the number of timesteps in the simulation. The same is true for any static quantity for which a scaling formula analogous to equations (4.51) and (4.52) can be derived.

The results for heat capacity are not nearly as smooth as those for energy or pressure. In fact, for $M=864$ and $M=500$, the degree of noise was so extreme that the results were rendered useless. Thus, Figures 4.16 and 4.17 contain only the data from the $M=2916$, $M=2048$, and $M=1372$ reduced systems. From these, the only conclusions that can be drawn are that the results are the right order of magnitude, and that neither model correctly predicts the divergence of $C_{V}$ near the phase transition. This latter fact should come as no surprise, given the explanation outlined above.

There are two main reasons why any calculation of the heat capacity via these methods will be inherently prone to noise. The first, as mentioned in Section 4.5.1, is that $C_{V}$ represents the fluctuation of a quantity, rather than just a simple average. More important, 


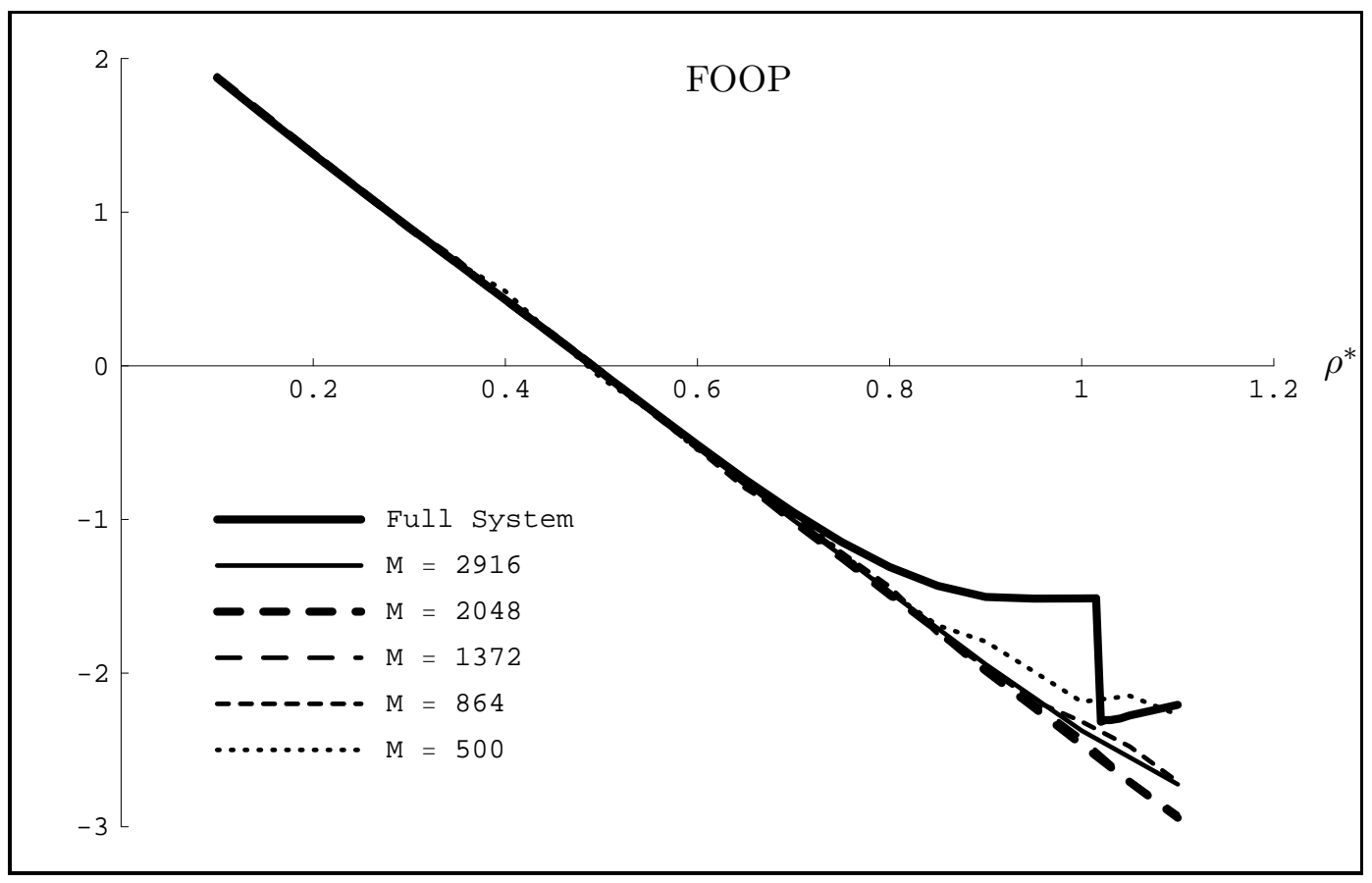

Figure 4.12: $E^{*} / N$ of the full system, and $\widehat{E}^{*}(M) / N$ calculated via the FOOP system for various $M$ values.

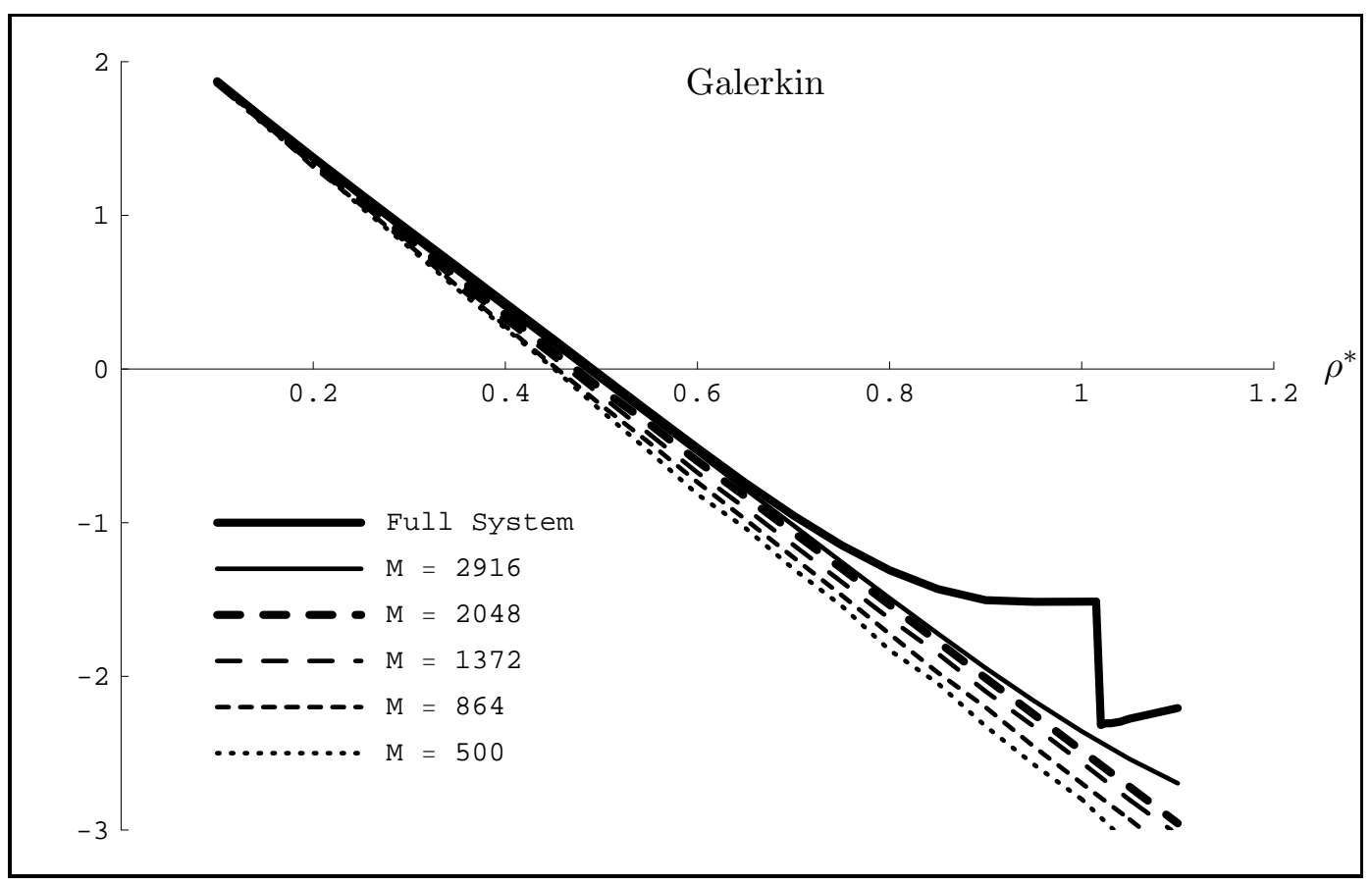

Figure 4.13: $E^{*} / N$ of the full system, and $\widehat{E}^{*}(M) / N$ calculated via the Galerkin system for various $M$ values. 


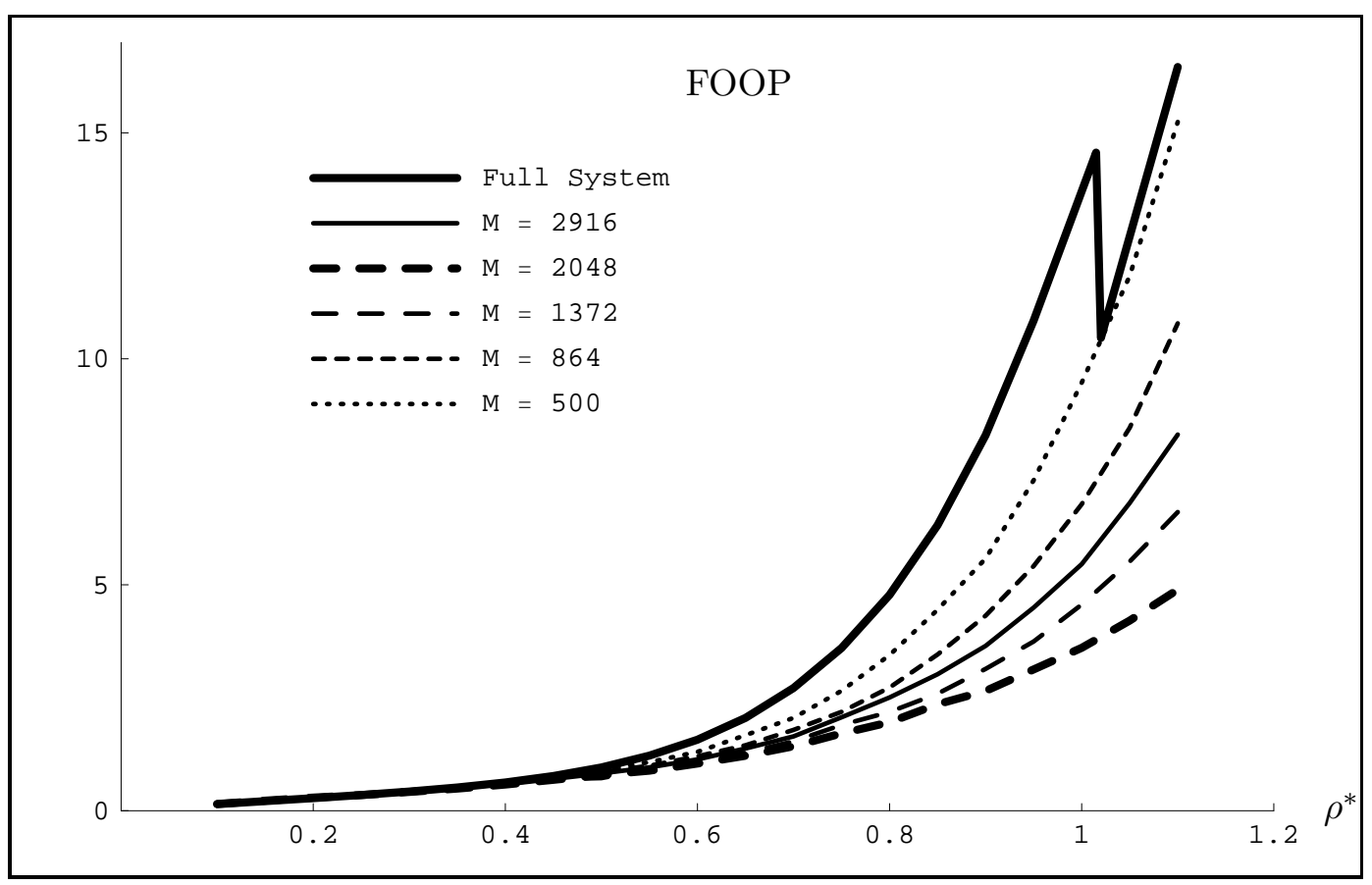

Figure 4.14: $P^{*}$ of the full system, and $\widehat{P}^{*}(M)$ calculated via the FOOP system for various $M$ values.

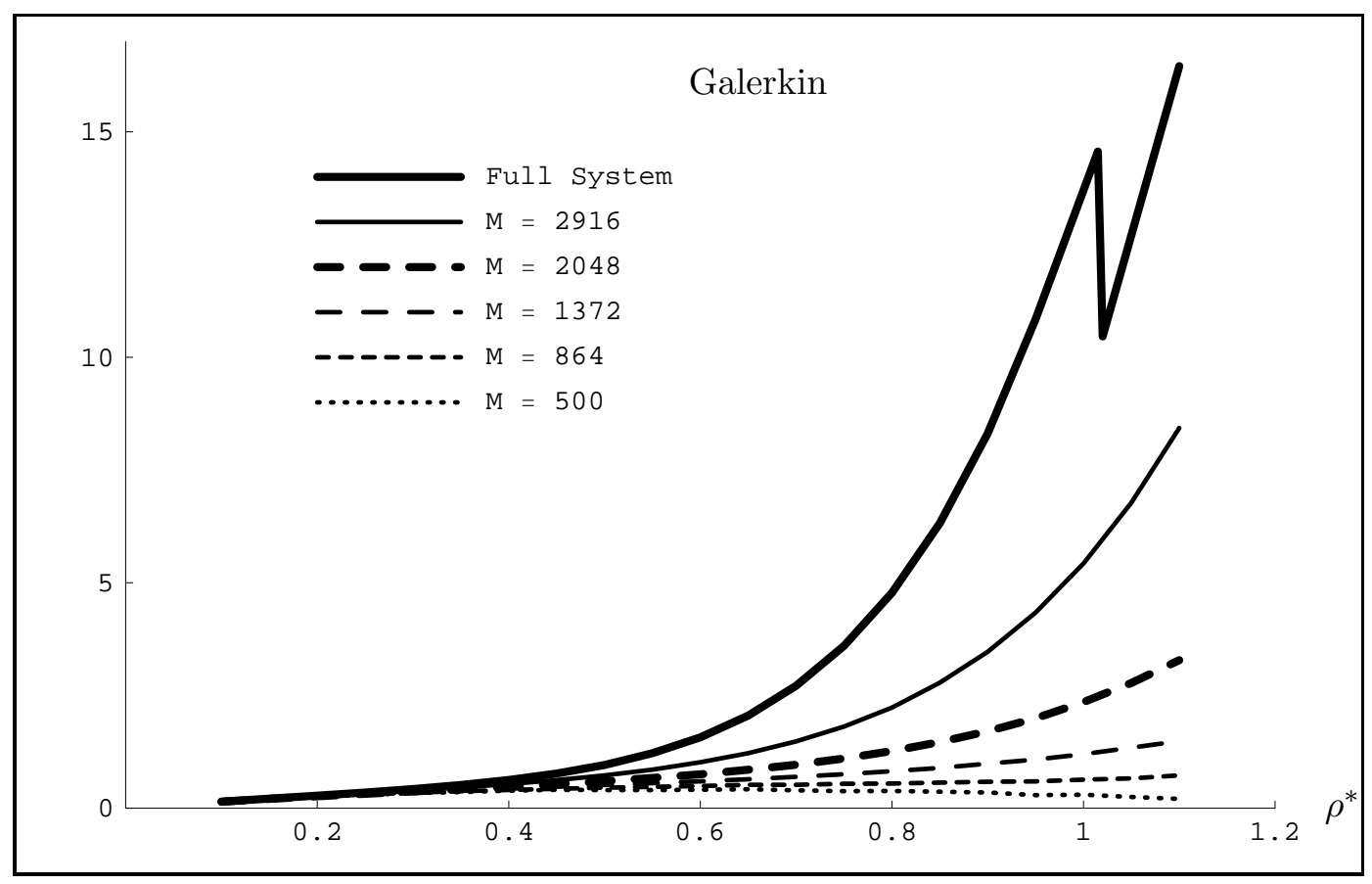

Figure 4.15: $P^{*}$ of the full system, and $\widehat{P}^{*}(M)$ calculated via the Galerkin system for various $M$ values. 


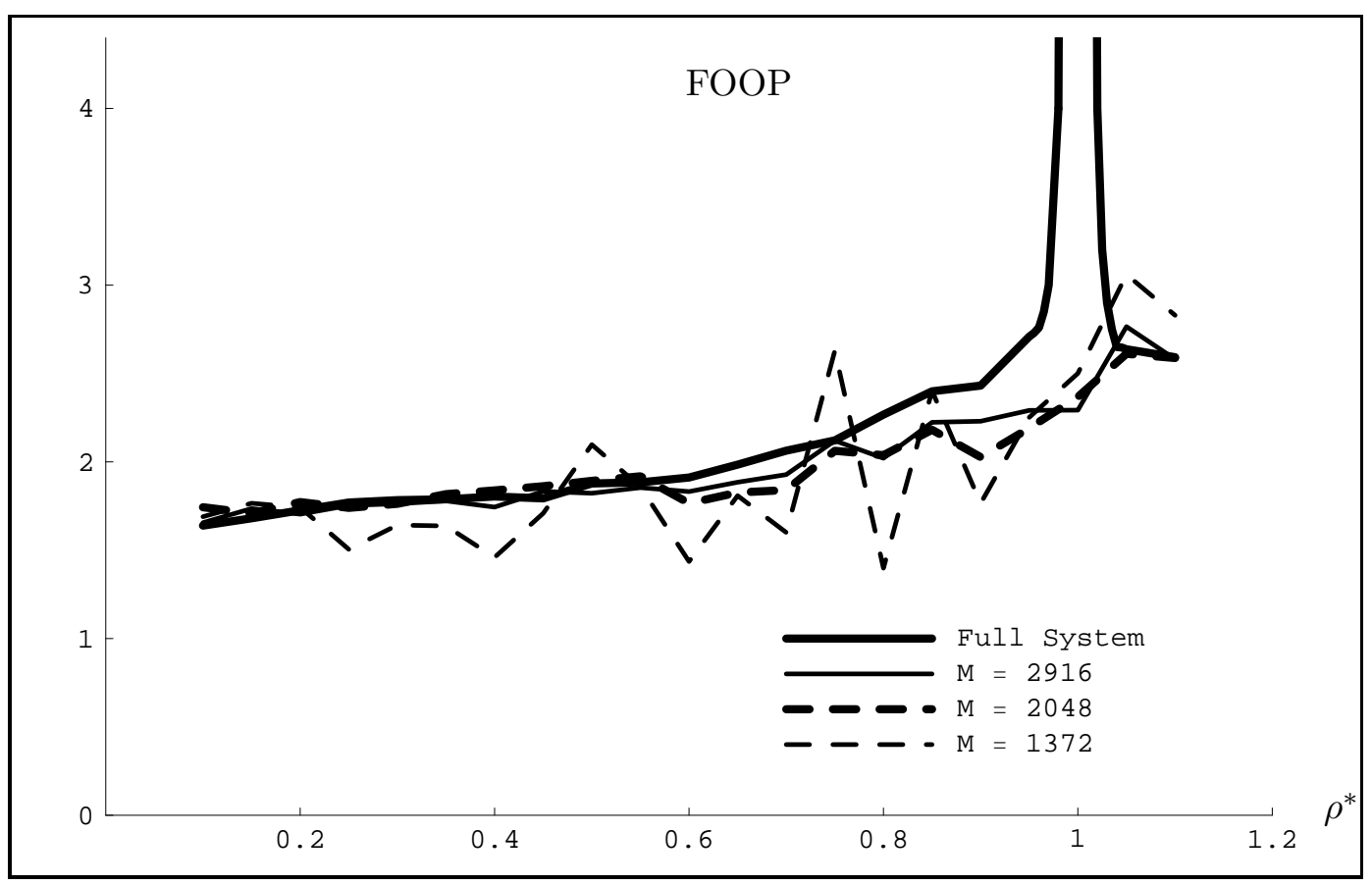

Figure 4.16: $C_{V}^{*} / N$ of the full system, and $\widehat{C}_{V}^{*}(M) / N$ calculated via the FOOP system for various $M$ values.

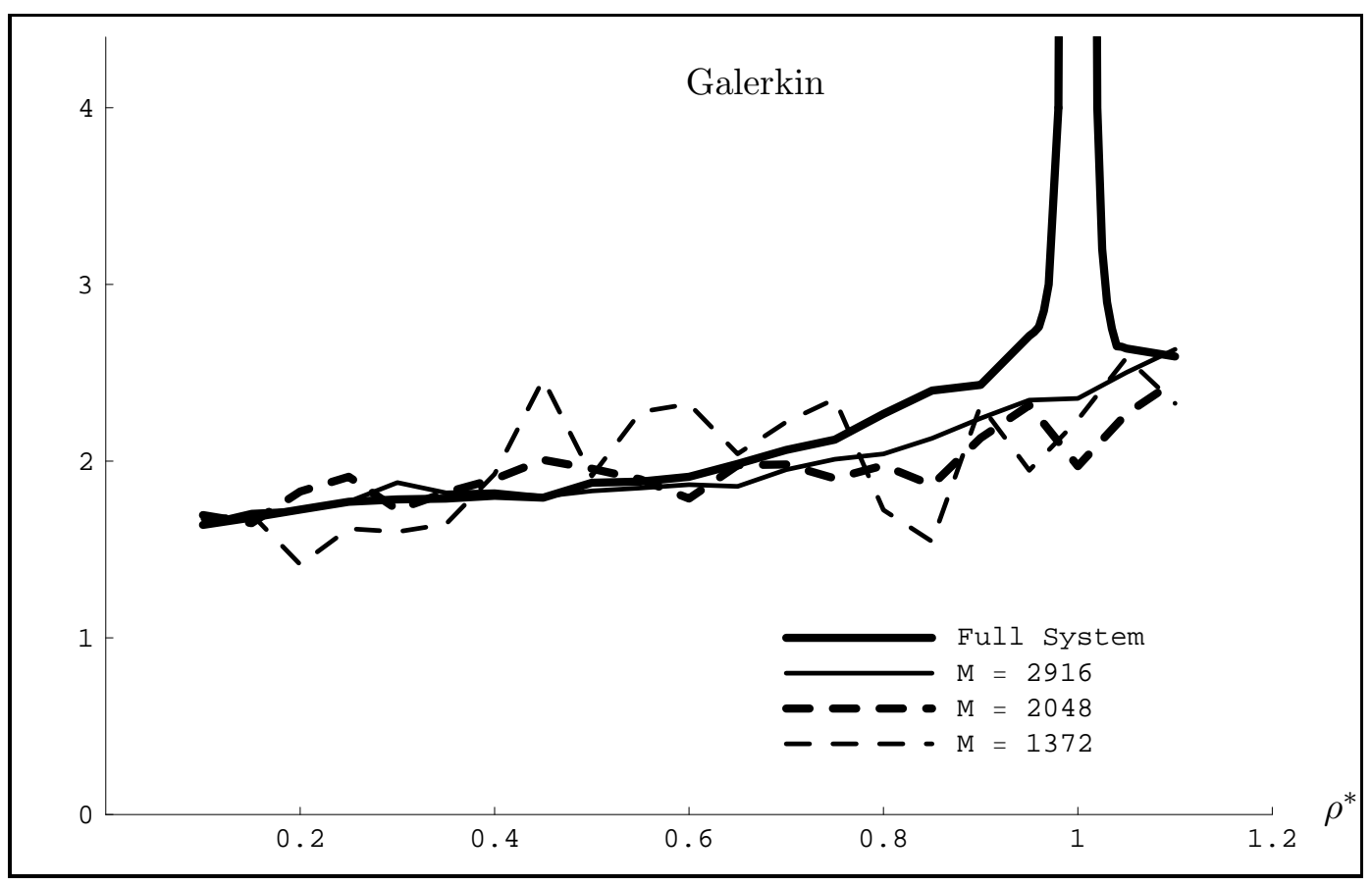

Figure 4.17: $C_{V}^{*} / N$ of the full system, and $\widehat{C}_{V}^{*}(M) / N$ calculated via the Galerkin system for various $M$ values. 
however, is the fact, alluded to briefly in Section 4.3.1, that the accuracy of the expression (4.57) for $\widehat{C}_{V}(M)$ depends upon a large degree of cancellation between $\left\langle\phi^{2}\right\rangle$ and $\langle\phi\rangle^{2}$. In particular, the last two terms in (4.57) (i.e. those involving alpha 3 and $\phi_{r r}$ ) are of similar magnitude but opposite sign. Each of these two terms has a coefficient of order $(N / M)^{4}$, which can be as high as $\sim 4000$ for the values of $N$ and $M$ we are considering. In general, any calculation that involves finding the difference between two large random quantities that nearly cancel one another will be subject to overwhelming uncertainty. Unfortunately, there is no remedy for this problem aside from carrying out simulations much longer than those performed for this thesis.

Unlike the heat capacity data, the energy and pressure results are regular enough to permit some discussion of the magnitude their errors. We define $\delta E$ to be the deviation between the energy per particle recovered from the reduced system and the full system:

$$
\delta E \equiv\left|\frac{\widehat{E}(M)-E}{N}\right| .
$$

The deviation in the pressure is similarly defined as

$$
\delta P \equiv|\widehat{P}(M)-P|
$$

Figures 4.18 and 4.19 show $\delta E$ as a function of $M$ at several moderate-to-high densities for FOOP and the Galerkin system, respectively. Similar graphs for $\delta P$ are shown in Figures 4.20 and 4.21. As predicted in Section 4.4, we see that for FOOP both $\delta E$ and $\delta P$ have a maximum in the intermediate region near $M=2048$. This effect is most striking in the results for $\delta P$. By contrast, the Galerkin data shows no such feature; both $\delta E$ and $\delta P$ increase monotonically as $M$ decreases. This is because the Galerkin system contains no mechanism to compensate for the neglected particles, as FOOP does in the form of a modified pair potential.

It should be pointed out that for low values of $\rho^{*}$, which are not shown in these figures, FOOP no longer produces these types of neat, unimodal error curves. This is because, at low densities, the systematic error associated with neglecting many-particle effects is negligible compared to the various other sources of random noise described earlier.

\section{Autocorrelation Functions}

The velocity autocorrelation functions (VAC's) of the full system and of the various FOOP systems are shown in Figure 4.22 for several representative values of $\rho^{*}$. As predicted in Section 4.3.2, FOOP does not do nearly as well calculating autocorrelation functions as it does with quantities like energy or pressure. We see that, although the value of $C_{\widehat{v} \widehat{v}}(t)$ is constrained to equal $k_{B} T / m$ at $t=0$, FOOP fails to capture the correct shape of the VAC's for $t>0$, especially when $M$ is small. At low densities, the decay rate is insufficient, whereas at high densities, FOOP does not reflect the oscillatory behavior of the full system's VAC, which is typical of a solid or dense fluid. It should be mentioned that the VAC's calculated from the Galerkin system (which are not shown) were found to be identical to those generated by FOOP, which implies that the difference between the renormalized and bare potentials does not significantly impact autocorrelation functions.

The shear stress autocorrelation functions (SSAC's), shown in Figure 4.23, were calculated using (3.14) and (4.59). These are subject to far greater statistical errors than the 


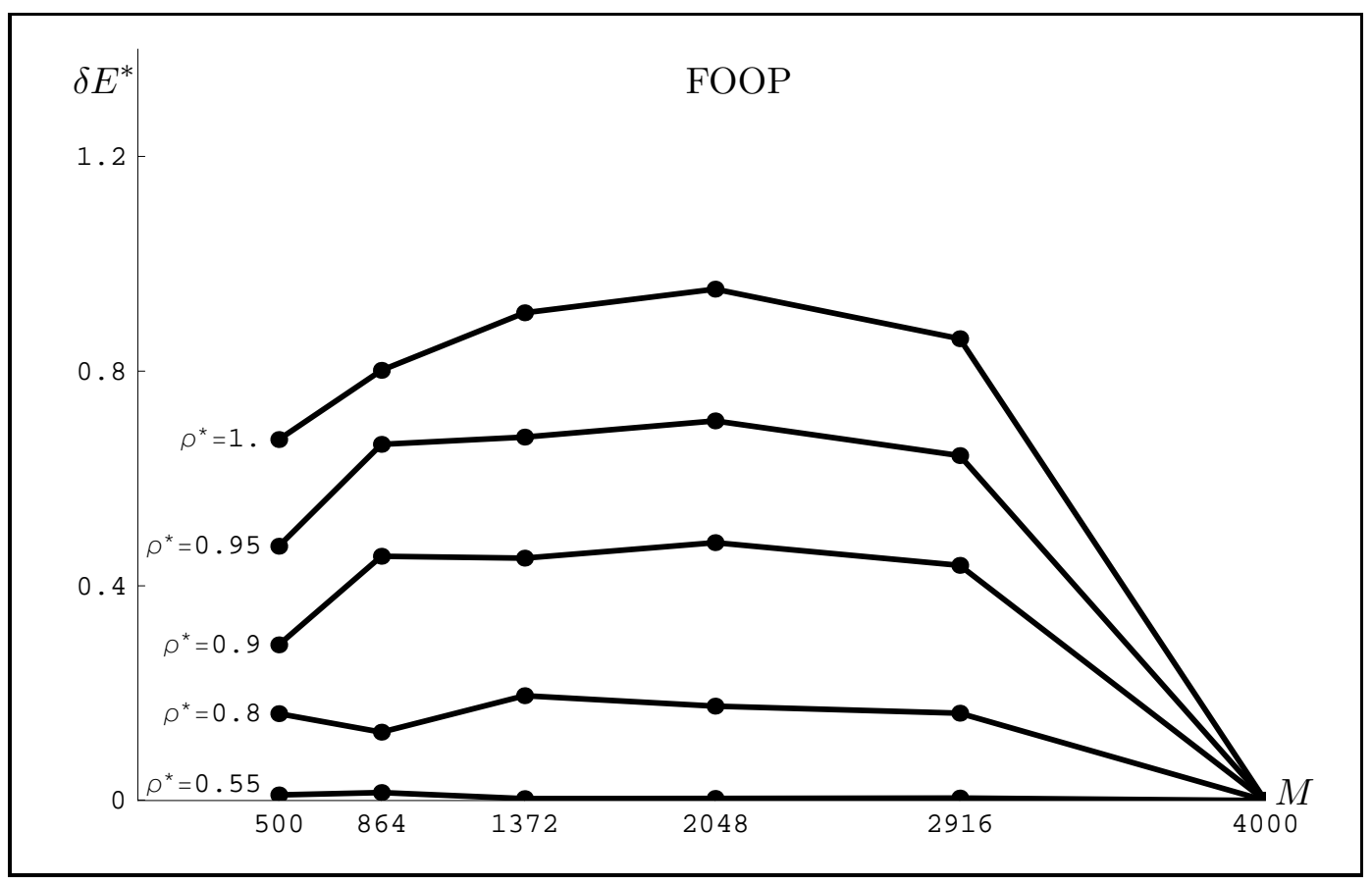

Figure 4.18: $\delta E^{*}$ of the FOOP system as a function of $M$, for several densities.

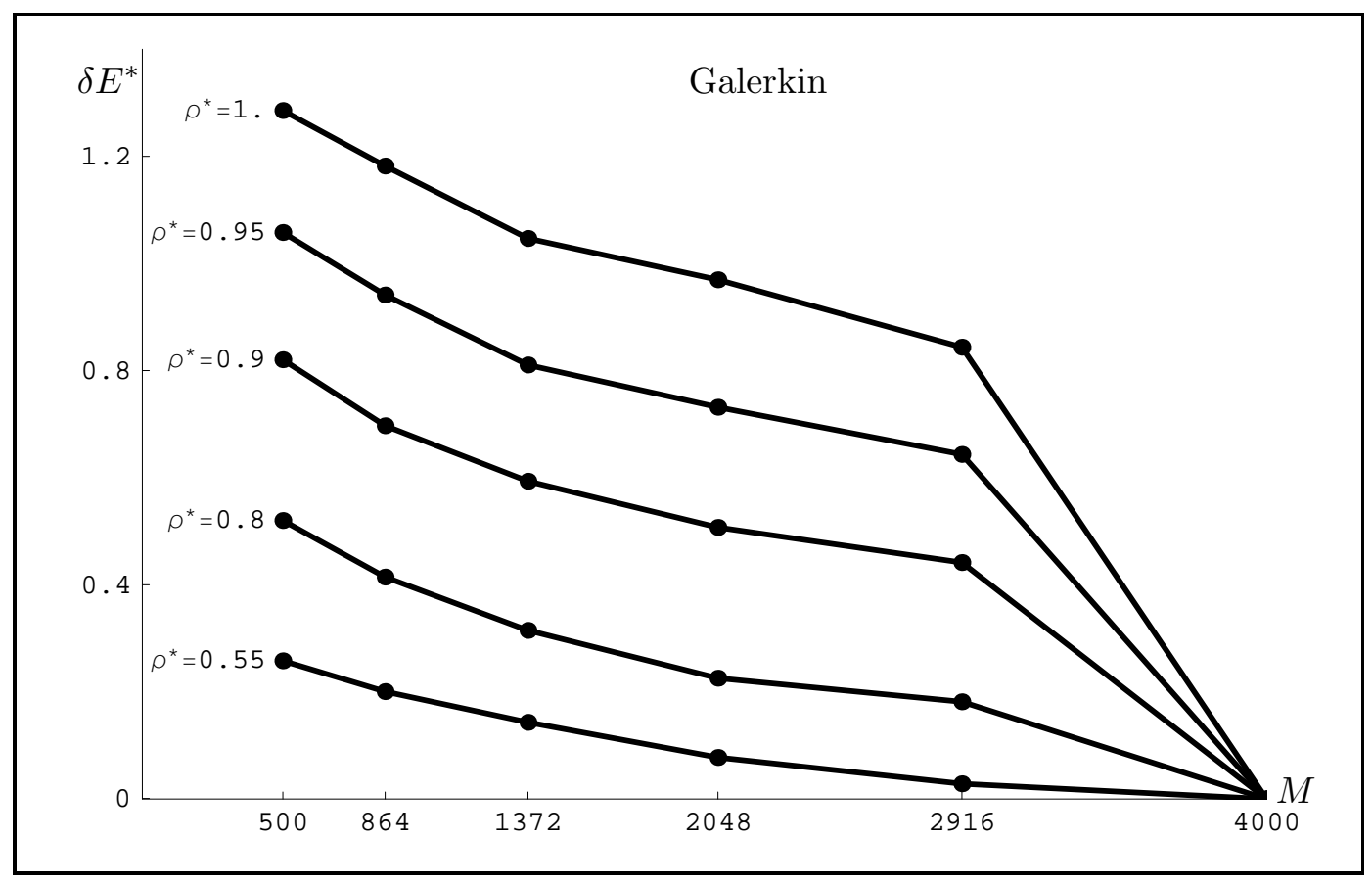

Figure 4.19: $\delta E^{*}$ of the Galerkin system as a function of $M$, for several densities. 


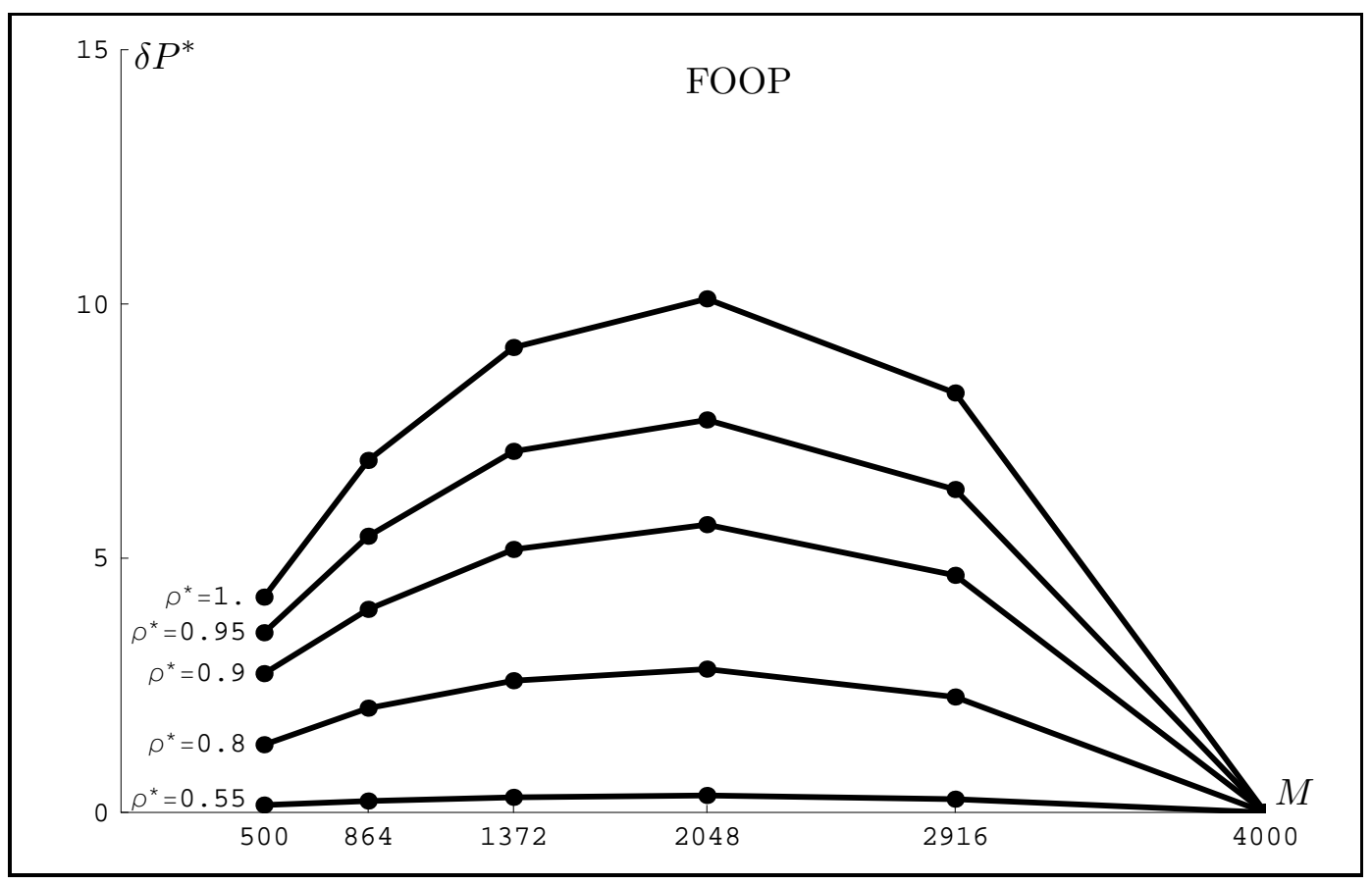

Figure 4.20: $\delta P^{*}$ of the FOOP system as a function of $M$, for several densities.

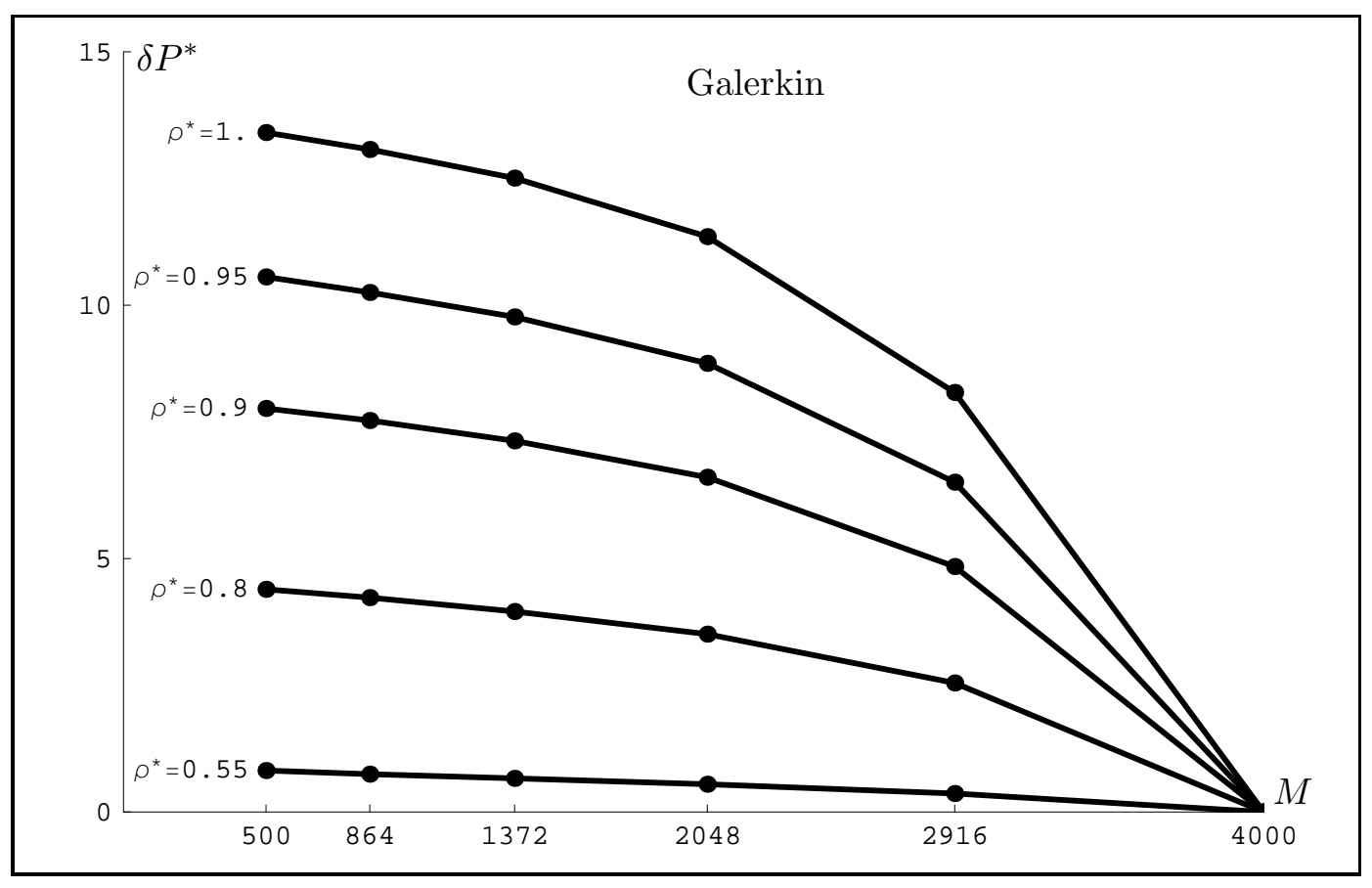

Figure 4.21: $\delta P^{*}$ of the Galerkin system as a function of $M$, for several densities. 
VAC's, due to the fact that the definitions of $C_{\Xi \Xi}(t)$ and $C_{\widehat{\Xi} \widehat{\Xi}}(t)$ do not allow us to perform an average over all particles for each value of $t$, as we could when calculating the VAC's. We are then left only with an average over all timesteps, which yields a precision an order of magnitude smaller than in the VAC case. Despite the increased noise, however, several features are clear. Aside from random errors, there are two types of deviations that can be discerned between the SSAC's of the FOOP and full systems. First, we see that the reduced and full system SSAC's do not coincide very well at $t=0$ for high densities. This is again due to our neglect of many-particle effects, since the value of the autocorrelation function of any quantity at $t=0$ is the same as the static average of that quantity's square, i.e. $C_{A A}(0)=\langle A(0) A(0)\rangle=\left\langle A^{2}\right\rangle$. Also apparent, particularly in the low density result, is the fact that the reduced SSAC's do not decay sufficiently fast to match the behavior of the full system. This is in accord with the VAC results described above.

Clearly, there is room to improve upon FOOP's treatment of autocorrelation functions. In order to better calculate these quantities, we must find some way of accounting for the contributions of the memory and forcing terms in (3.7). This will be the topic of Chapter 5 . 


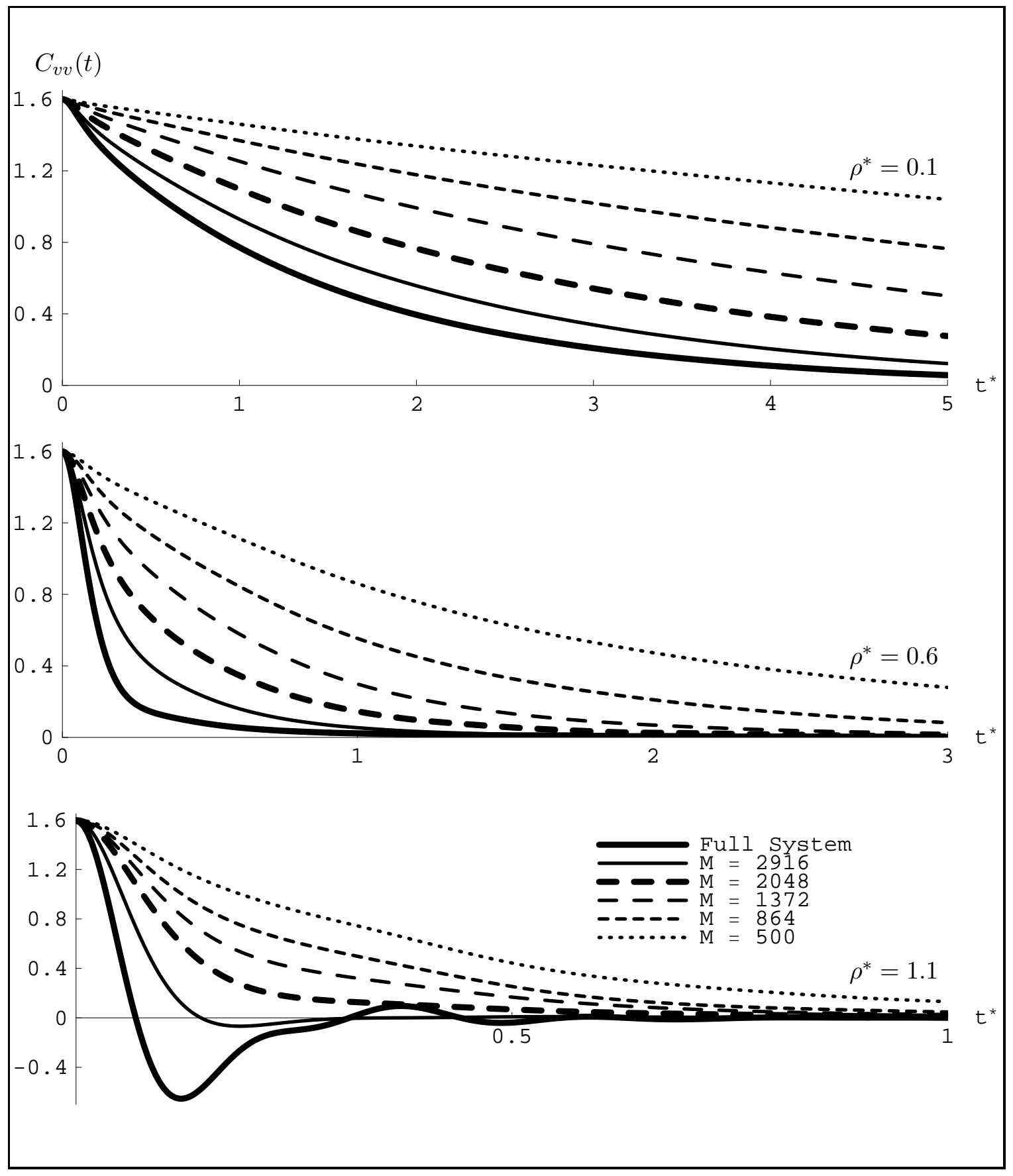

Figure 4.22: Velocity autocorrelation functions calculated via the full system and via FOOP, for various values of $M$, and for several densities. 


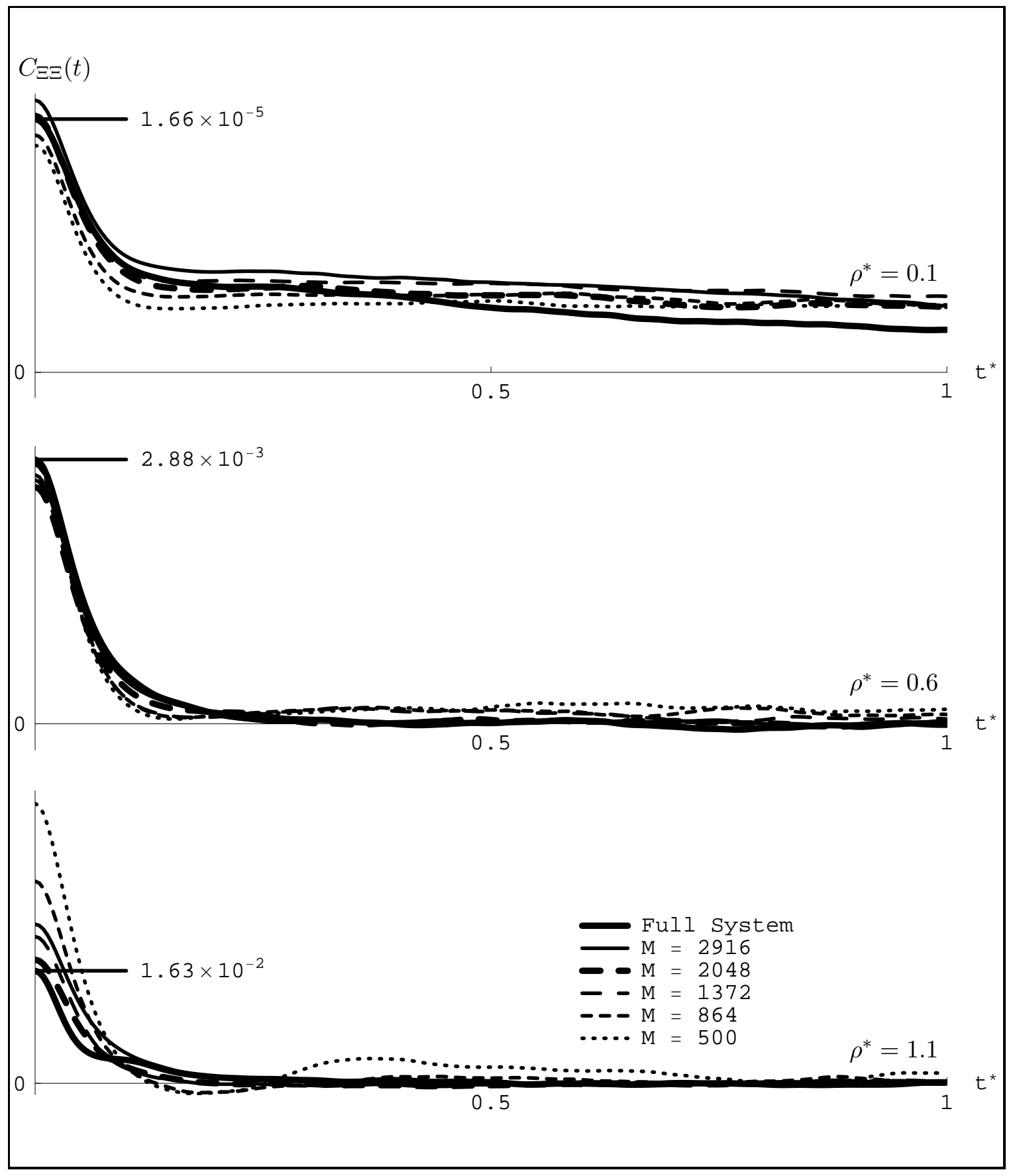

Figure 4.23: Shear stress autocorrelation functions calculated via the full system and via FOOP, for various values of $M$, and for several densities. The values of $C_{\Xi \Xi}(0)$ for the full system are labeled for reference. 


\section{Chapter 5}

\section{The Linear Friction Approximation}

In order to include the effects of the memory integral in (3.7), we propose a linear friction approximation to this term. This leads to a Fokker-Planck equation for the evolution of the probability density of the positions and momenta. If we then require the renormalized canonical distribution (4.49) to be a stationary solution of this equation, we can derive constraints on the form of the random forcing term. The resulting system is a set of coupled Langevin equations for the resolved positions and momenta.

\subsection{Approximating the Memory Kernel}

In Chapter 4, the FOOP approximation was unable to accurately reproduce the shape of the autocorrelation functions of dynamical variables. In order to remedy this, we need to somehow include the contributions from the memory and forcing terms in (3.7). We begin by developing an approximation to the memory integral associated with the $i^{\text {th }}$ particle, which is given exactly by

$$
\mathcal{M}_{i}(t) \equiv \int_{0}^{t} d s e^{(t-s) L} \mathscr{K}_{i}(\widehat{x}, \widehat{y}, s)=\int_{0}^{t} d s \mathscr{K}_{i}(\widehat{q}(t-s), \widehat{p}(t-s), s),
$$

where

$$
\mathscr{K}_{i}(\widehat{x}, \widehat{y}, s)=\mathbb{P} L e^{s \mathbb{Q} L} \mathbb{Q} L \widehat{y}_{i} .
$$

(Recall that $\widehat{y}_{i}$ is the initial value of the momentum of the $i^{\text {th }}$ resolved particle.) The definition of $\mathcal{M}_{i}(t)$ implies that the integration variable $s$ can be interpreted as increasing backwards in time, with larger values of $s$ incorporating information about the behavior of the resolved variables further in the past. Since the memory kernel (5.2) contains a leading factor of $\mathbb{P}$ and the dynamics are highly nonlinear, we expect the system to "forget" about the values of the resolved variables at much earlier times. This implies that $\mathscr{K}_{i}(\widehat{x}, \widehat{y}, s) \rightarrow 0$ as $s \rightarrow \infty$ for all finite $\widehat{x}$ and $\widehat{y}$. Furthermore, since we assumed in Chapter 3 that our interparticle potential is bounded from below, conservation of energy implies that the trajectories $(\widehat{q}(t), \widehat{p}(t))$ are bounded. Thus, we should expect the integrand $\mathscr{K}_{i}(\widehat{q}(t-s), \widehat{p}(t-s), s)$ to decay to zero for large $s$ as well.

We now make the additional assumption that the support backwards in time of the memory kernel $\mathscr{K}_{i}(\widehat{x}, \widehat{y}, s)$ is short, with a characteristic timescale $\tau>0$, and that we may approximate it by

$$
\mathscr{K}_{i}(\widehat{x}, \widehat{y}, s) \approx \mathscr{K}_{i}(\widehat{x}, \widehat{y}, 0) k(s / \tau) .
$$


Here $k(s / \tau)$ is an unknown function that must satisfy $k(0)=1$ and $\int_{0}^{\infty} d s k(s / \tau)=\tau$. The approximation (5.3) is common in the nonequilibrium statistical mechanics literature $[39,53,59]$, and it often yields surprisingly good results for autocorrelation functions. This is due to the fact that correlation functions are somewhat insensitive to the exact form of the memory kernel. Some common choices for $k(s / \tau)$ include an exponential form, $k(s / \tau)=\exp (-s / \tau)$, as well as a Gaussian form, $k(s / \tau)=\exp \left(-\frac{\pi}{4} \frac{s^{2}}{\tau^{2}}\right)$. Since $\tau$ is a timescale of the microscopic dynamics, it should be an intensive quantity, which may depend on $\widehat{\rho}, \widetilde{\rho}$, and $T$, but not on $N, M$, or $V$ separately. Aside from these rather general observations, $\tau$ remains an undetermined parameter. Some criteria for estimating the best value of $\tau$ will be discussed later in this chapter.

The memory term now becomes

$$
\mathcal{M}_{i}(t) \approx \int_{0}^{t} d s e^{(t-s) L} \mathscr{K}_{i}(\widehat{x}, \widehat{y}, 0) k(s / \tau) .
$$

Regardless of the exact form of $k(s / \tau)$, we assume that $\tau$ is small, which implies that $k(s / \tau)$ is strongly peaked near $s=0$. We then may make the approximation that

$$
\mathcal{M}_{i}(t) \approx \tau e^{t L} \mathscr{K}_{i}(\widehat{x}, \widehat{y}, 0)
$$

This is equivalent to approximating the integral (5.1) by its value at $s=0$ multiplied by the constant timescale $\tau$. As we will see in Section 5.2, (5.4) implies that the random forcing term in (3.7) is $\delta$-correlated in time. Thus, (5.4) is closely related to the assumption of $\delta$-correlated noise that is often found in the literature of dynamical renormalization [24].

In general, (5.4) is difficult to justify analytically, although, for the special case where $k(s / \tau)=\exp (-s / \tau)$, we can derive (5.4) in the low- $\tau$ limit by showing that

$$
\lim _{\tau \rightarrow 0}\left|\frac{\int_{0}^{t} d s \mathscr{K}_{i}(t-s) e^{-s / \tau}-\tau \mathscr{K}_{i}(t)}{\tau}\right|=0
$$

when $t>0$. Here, we have used the shorthand $\mathscr{K}_{i}(t)=e^{t L} \mathscr{K}_{i}(\widehat{x}, \widehat{y}, 0)$. We proceed as follows:

$$
\begin{aligned}
\frac{1}{\tau}\left[\int_{0}^{t} d s \mathscr{K}_{i}(t-s) e^{-s / \tau}-\tau \mathscr{K}_{i}(t)\right] & =\int_{0}^{t} d s \mathscr{K}_{i}(t-s) \frac{d}{d s}\left(-e^{-s / \tau}\right)-\mathscr{K}_{i}(t) \\
& =-\left.\mathscr{K}_{i}(t-s) e^{-s / \tau}\right|_{s=0} ^{t}-\mathscr{K}_{i}(t)+\int_{0}^{t} d s e^{-s / \tau} \frac{d}{d s} \mathscr{K}_{i}(t-s) \\
& =-\mathscr{K}_{i}(0) e^{-t / \tau}-\int_{0}^{t} d s e^{-s / \tau} \mathscr{K}_{i}^{\prime}(t-s) .
\end{aligned}
$$

If we also assume that $\left|\mathscr{K}_{i}^{\prime}(t)\right| \leq C$ for some $C>0$, then (5.5) becomes:

$$
\begin{aligned}
\lim _{\tau \rightarrow 0}\left|\frac{\int_{0}^{t} d s \mathscr{K}_{i}(t-s) e^{-s / \tau}-\tau \mathscr{K}_{i}(t)}{\tau}\right| & \leq \lim _{\tau \rightarrow 0}\left[\left|\mathscr{K}_{i}(0)\right| e^{-t / \tau}+C \int_{0}^{t} d s e^{-s / \tau}\right] \\
& \leq \lim _{\tau \rightarrow 0}\left[\left|\mathscr{K}_{i}(0)\right| e^{-t / \tau}+C \tau\left(1-e^{-t / \tau}\right)\right]=0 .
\end{aligned}
$$

This shows that, in this instance at least, (5.4) holds to lowest order in $\tau$. 
Equation (5.4) requires us to evaluate $\mathscr{K}_{i}(\widehat{x}, \widehat{y}, 0)$. According to (5.2), this quantity is

$$
\begin{aligned}
\mathscr{K}_{i}(\widehat{x}, \widehat{y}, 0) & =\mathbb{P} L \mathbb{Q} L \widehat{y}_{i} \\
& =\mathbb{P} L(I-\mathbb{P})\left(-\nabla_{\widehat{x}_{i}} \phi(x)\right) \\
& =-\mathbb{P} \sum_{j=1}^{N} \frac{1}{m} y_{j} \cdot \nabla_{x_{j}}\left(\nabla_{\widehat{x}_{i}} \phi(x)-\nabla_{\widehat{x}_{i}} \widehat{\phi}(\widehat{x})\right) \\
& =-\sum_{j=1}^{N} \frac{1}{m} \mathbb{P}\left\{y_{j} \cdot \nabla_{x_{j}}\left(\nabla_{\widehat{x}_{i}} \phi(x)-\nabla_{\widehat{x}_{i}} \widehat{\phi}(\widehat{x})\right)\right\} .
\end{aligned}
$$

Since the Hamiltonian (3.1) of the full system is separable into a kinetic part and a potential part, the projection operator $\mathbb{P}$ is separable as well. This allows us to write (5.6) as

$$
\mathscr{K}_{i}(\widehat{x}, \widehat{y}, 0)=-\sum_{j=1}^{N} \frac{1}{m} \mathbb{P}\left\{\mathbb{P}\left\{y_{j}\right\} \cdot \nabla_{x_{j}}\left(\nabla_{\widehat{x}_{i}} \phi(x)-\nabla_{\widehat{x}_{i}} \widehat{\phi}(\widehat{x})\right)\right\} .
$$

The fact that the $\widetilde{y}_{i}$ have zero mean implies that

$$
\mathbb{P} y_{j}= \begin{cases}\widehat{y}_{j} & 1 \leq j \leq M \\ 0 & j>M\end{cases}
$$

in which case we can truncate the sum in (5.7) at the $M^{\text {th }}$ term, to yield

$$
\mathscr{K}_{i}(\widehat{x}, \widehat{y}, 0)=-\sum_{j=1}^{M} \frac{1}{m} \mathbb{P}\left\{\left(\nabla_{\widehat{x}_{i}} \otimes \nabla_{\widehat{x}_{j}}\right)(\phi(x)-\widehat{\phi}(\widehat{x}))\right\} \widehat{y}_{j} .
$$

Here " $\otimes$ " is the dyadic product, which, given two vectors $a$ and $b$, forms a matrix with components $(a \otimes b)_{\mu \nu}=a_{\mu} b_{\nu}$. When this matrix acts on a third vector $c$, the result is $(a \otimes b) c=(b \cdot c) a$.

We now define the matrices $A^{i j}$ by

$$
A^{i j}(\widehat{x}) \equiv \frac{\tau}{m} \mathbb{P}\left\{\left(\nabla_{\widehat{x}_{i}} \otimes \nabla_{\widehat{x}_{j}}\right)(\phi(x)-\widehat{\phi}(\widehat{x}))\right\},
$$

in which case the memory term becomes

$$
\mathcal{M}_{i}(t) \approx-\sum_{j=1}^{M} A^{i j}(\widehat{q}) \widehat{p}_{j}
$$

This has the form of a linear frictional force on particle $i$ that depends on the momenta of all $M$ resolved particles, with matrix coefficients $A^{i j}$ that are functions of the configuration $\widehat{q}$. The $A^{i j}$ are therefore called the friction matrices.

We should be careful to note that $A^{i j}$ is not the $i, j^{\text {th }}$ component of a matrix $A$. Rather, the $A^{i j}$ are a family of $d \times d$ matrices, indexed by $i$ and $j$, which range from 1 to $M$. If we wish to refer to the components of $A^{i j}$, we will use Greek subscript indices: $A_{\mu \nu}^{i j}$. According to the definition of the dyadic product, $(a \otimes b)^{T}=(b \otimes a)$, where the superscript ${ }^{T}$ indicates a matrix transpose. This implies that $\left(A^{i j}\right)^{T}=A^{j i}$. Furthermore, another property of the $A^{i j}$ is described by the following theorem: 
Theorem 5.1 Given an arbitrary set of $M$ d-dimensional vectors $a_{i}$, the following inequality holds:

$$
\sum_{i, j=1}^{M} a_{i} \cdot\left(A^{i j}(\widehat{x}) a_{j}\right) \geq 0
$$

Proof: In order to prove this, we must first obtain the $A^{i j}$ in a slightly different form than (5.8):

$$
\begin{aligned}
A^{i j}(\widehat{x}) & =\frac{\tau}{m} \mathbb{P}\left\{\left(\nabla_{\widehat{x}_{i}} \otimes \nabla_{\widehat{x}_{j}}\right)(\phi(x)-\widehat{\phi}(\widehat{x}))\right\} \\
& =\frac{\tau}{m} \sum_{\mu, \nu=1}^{d}\left(\hat{e}_{\mu} \otimes \hat{e}_{\nu}\right) \frac{\int d \widetilde{x}\left[\nabla_{i \mu} \nabla_{j \nu}(\phi-\widehat{\phi})\right] e^{-\beta \phi}}{\int d \widetilde{x} e^{\beta \phi}} .
\end{aligned}
$$

where " $\nabla_{i \mu}$ " is short for $\nabla_{\widehat{x}_{i \mu}}$, and $\hat{e}_{\mu}$ is a unit vector in the $\mu^{\text {th }}$ direction. The integral above can be manipulated as follows:

$$
\begin{aligned}
& \int \frac{d \widetilde{x}\left[\nabla_{i \mu} \nabla_{j \nu}(\phi-\widehat{\phi})\right] e^{-\beta \phi}}{\int d \widetilde{x} e^{-\beta \phi}} \\
&= \frac{\int d \widetilde{x}\left(\nabla_{i \mu} \nabla_{j \nu} \phi\right) e^{-\beta \phi}}{\int d \widetilde{x} e^{-\beta \phi}}-\nabla_{i \mu} \nabla_{j \nu} \widehat{\phi} \\
&= \nabla_{i \mu}\left(\frac{\int d \widetilde{x}\left(\nabla_{j \nu} \phi\right) e^{-\beta \phi}}{\int d \widetilde{x} e^{-\beta \phi}}\right)+\beta \frac{\int d \widetilde{x}\left(\nabla_{j \nu} \phi\right)\left(\nabla_{i \mu} \phi\right) e^{-\beta \phi}}{\int d \widetilde{x} e^{-\beta \phi}} \\
&-\beta\left(\frac{\int d \widetilde{x}\left(\nabla_{j \nu} \phi\right) e^{-\beta \phi}}{\int d \widetilde{x} e^{-\beta \phi}}\right)\left(\frac{\int d \widetilde{x}\left(\nabla_{i \mu} \phi\right) e^{-\beta \phi}}{\int d \widetilde{x} e^{-\beta \phi}}\right)-\nabla_{i \mu} \nabla_{j \nu} \widehat{\phi} \\
&=\nabla_{i \mu} \nabla_{j \nu} \widehat{\phi}+\beta \mathbb{P}\left\{\left(\nabla_{i \mu} \phi\right)\left(\nabla_{j \nu} \phi\right)\right\}-\beta\left(\nabla_{i \mu} \widehat{\phi}\right)\left(\nabla_{j \nu} \widehat{\phi}\right)-\nabla_{i \mu} \nabla_{j \nu} \widehat{\phi} \\
&=\frac{1}{k_{B} T} \mathbb{P}\left\{\nabla_{i \mu}(\phi-\widehat{\phi}) \nabla_{j \nu}(\phi-\widehat{\phi})\right\},
\end{aligned}
$$

which allows us to write $A^{i j}$ as

$$
A^{i j}(\widehat{x})=\frac{\tau}{m k_{B} T} \mathbb{P}\left\{\left[\nabla_{\widehat{x}_{i}}(\phi(x)-\widehat{\phi}(\widehat{x}))\right] \otimes\left[\nabla_{\widehat{x}_{j}}(\phi(x)-\widehat{\phi}(\widehat{x}))\right]\right\} .
$$

Given this form for $A^{i j}$, and $M$ arbitrary, $d$-dimensional vectors $a_{i}$ (which may or may not depend on the resolved variables $\widehat{x}$ and $\widehat{y}$ ), we have:

$$
\begin{aligned}
\sum_{i, j=1}^{M} a_{i} \cdot\left(A^{i j}(\widehat{x}) a_{j}\right) & =\frac{\tau}{m k_{B} T} \sum_{i, j=1}^{M} \mathbb{P}\left\{\left[a_{i} \cdot \nabla_{\widehat{x}_{i}}(\phi(x)-\widehat{\phi}(\widehat{x}))\right]\left[a_{j} \cdot \nabla_{\widehat{x}_{j}}(\phi(x)-\widehat{\phi}(\widehat{x}))\right]\right\} \\
& =\frac{\tau}{m k_{B} T} \mathbb{P}\left\{\left[\sum_{i=1}^{M} a_{i} \cdot \nabla_{\widehat{x}_{i}}(\phi(x)-\widehat{\phi}(\widehat{x}))\right]^{2}\right\} \geq 0
\end{aligned}
$$


Theorem $5.1^{1}$ is equivalent to the statement that $\mathcal{A}$ is positive semi-definite, where

$$
\mathcal{A}=\left(\begin{array}{cccc}
A^{11} & A^{12} & \cdots & A^{1 M} \\
A^{21} & A^{22} & & \vdots \\
\vdots & & \ddots & \vdots \\
A^{M 1} & \cdots & \cdots & A^{M M}
\end{array}\right)
$$

is the $M d \times M d$ block matrix of the $A^{i j}$. The fact that $\left(A^{i j}\right)^{T}=A^{j i}$ also tells us that $\mathcal{A}^{T}=\mathcal{A}$.

\subsection{Fokker-Planck Equations and the Forcing Term}

We have approximated the memory term in (3.7) by (5.9). We have yet to find a corresponding expression for the random forcing terms $\mathscr{F}_{i}(x, y, t)$. According to $(2.21)$, these are given exactly by

$$
\mathscr{F}_{i}(x, y, t)=e^{t \mathbb{Q} L} \mathbb{Q} L \widehat{y}_{i} .
$$

However, this expression is difficult to work with due to the leading $e^{t \mathbb{Q} L}$ factor, and so we will use a more indirect method of finding an approximation for the $\mathscr{F}_{i}$. First, some mathematical background must be introduced.

Suppose we have $n d$-dimensional, time-dependent vectors $z_{i}(t)$, which evolve according to the set of stochastic differential equations

$$
\frac{d}{d t} z_{i}=\Gamma_{i}(z, t)
$$

where the $\Gamma_{i}$ are stochastic processes that depend on the $z_{i}$. We now define the vectors $\alpha^{i}$ and the matrices $\gamma^{i j}$ by

$$
\begin{aligned}
\alpha^{i}(z, t) & \equiv \lim _{\Delta t \rightarrow 0} \frac{1}{\Delta t}\left\langle z_{i}(t+\Delta t)-z_{i}(t)\right\rangle \\
\gamma^{i j}(z, t) & \equiv \lim _{\Delta t \rightarrow 0} \frac{1}{\Delta t}\left\langle\left(z_{i}(t+\Delta t)-z_{i}(t)\right) \otimes\left(z_{j}(t+\Delta t)-z_{j}(t)\right)\right\rangle,
\end{aligned}
$$

where $\langle\ldots\rangle$ indicates an average over the ensemble of the $\Gamma_{i}$, treating the values of the $z_{i}$ at time $t$ as fixed. If the $\alpha^{i}$ and $\gamma^{i j}$ exist, then the probability density $f(z, t)$ of the $z_{i}$ evolves in time according to the Fokker-Planck equation [61]:

$$
\partial_{t} f+\sum_{i=1}^{n} \sum_{\mu=1}^{d} \partial_{z_{i \mu}}\left(\alpha_{\mu}^{i}(z, t) f\right)-\frac{1}{2} \sum_{i, j=1}^{n} \sum_{\mu, \nu=1}^{d} \partial_{z_{i \mu}} \partial_{z_{j \nu}}\left(\gamma_{\mu \nu}^{i j}(z, t) f\right)=0 .
$$

Example: Consider the quantity $u(t)$, which evolves according to the simple one-dimensional Langevin equation

$$
\frac{d}{d t} u=-a u+b \dot{W}(t)
$$

\footnotetext{
${ }^{1}$ Note that the proof of Theorem 5.1 presented here is similar in many respects to the proof in [9] of the decay of the renormalized Hamiltonian under the "t-damping approximation".
} 
where $a$ and $b$ are positive constants, and $\dot{W}(t)$ is the standard white noise process, which satisfies

$$
\langle\dot{W}(t)\rangle=0 \quad\left\langle\dot{W}(t) \dot{W}\left(t^{\prime}\right)\right\rangle=\delta\left(t-t^{\prime}\right) .
$$

The probability density $f(u, t)$ of $u$ at time $t$ satisfies

$$
\partial_{t} f+\partial_{u}(\alpha(u, t) f)-\frac{1}{2} \partial_{u}^{2}(\gamma(u, t) f)=0 .
$$

We can calculate $\alpha$ and $\gamma$ using the fact that

$$
u(t+\Delta t)=u(t)-a u(t) \Delta t+b \int_{t}^{t+\Delta t} d s \dot{W}(s)+O\left(\Delta t^{2}\right)
$$

in which case:

$$
\begin{aligned}
\alpha & =\lim _{\Delta t \rightarrow 0} \frac{1}{\Delta t}\langle u(t+\Delta t)-u(t)\rangle \\
& =\lim _{\Delta t \rightarrow 0} \frac{1}{\Delta t}\{-a u(t) \Delta t+b \int_{t}^{t+\Delta t} d s \underbrace{\langle\dot{W}(s)\rangle}_{=0}+O\left(\Delta t^{2}\right)\} \\
& =-a u \\
\gamma & =\lim _{\Delta t \rightarrow 0} \frac{1}{\Delta t}\langle(u(t+\Delta t)-u(t))(u(t+\Delta t)-u(t))\rangle \\
& =\lim _{\Delta t \rightarrow 0} \frac{1}{\Delta t}\left\langle\left(-a u(t) \Delta t+b \int_{t}^{t+\Delta t} d s \dot{W}(s)\right)\left(-a u(t) \Delta t+b \int_{t}^{t+\Delta t} d s^{\prime} \dot{W}\left(s^{\prime}\right)\right)+O\left(\Delta t^{2}\right)\right\rangle \\
& =\lim _{\Delta t \rightarrow 0} \frac{1}{\Delta t}\{a^{2} u^{2} \Delta t^{2}-2 a b u \Delta t \int_{t}^{t+\Delta t} d s \underbrace{\langle\dot{W}(s)\rangle}_{=0}+b^{2} \int_{t}^{t+\Delta t} d s \int_{t}^{t+\Delta t} d s^{\prime} \underbrace{\left\langle\dot{W}(s) \dot{W}\left(s^{\prime}\right)\right\rangle}_{=\delta\left(s-s^{\prime}\right)}\} \\
& =b^{2} .
\end{aligned}
$$

The Fokker-Planck equation (5.15) for $f(u, t)$ then becomes:

$$
\partial_{t} f-a \partial_{u}(u f)-\frac{1}{2} b^{2} \partial_{u}^{2} f=0 .
$$

The above techniques can also be used to generate a Fokker-Planck equation for the evolution of $f(\widehat{q}, \widehat{p}, t)$, the probability density function of the resolved variables. Our goal is to use this Fokker-Planck equation to derive some constraints on the form of the random forces $\mathscr{F}_{i}$. Given the approximation (5.9) to the memory term, the equations (3.7) for the evolution of the $\widehat{q}_{i}$ and $\widehat{p}_{i}$ become

$$
\begin{aligned}
\frac{d}{d t} \widehat{q}_{i} & =\frac{1}{m} \widehat{p}_{i} \\
\frac{d}{d t} \widehat{p}_{i} & =-\nabla_{\widehat{q}_{i}} \widehat{\phi}(\widehat{q})-\sum_{j=1}^{M} A^{i j}(\widehat{q}) \widehat{p}_{j}+\mathscr{F}_{i}(t) .
\end{aligned}
$$


We will treat the $\mathscr{F}_{i}$ as zero-mean stochastic processes with as-yet-unknown statistics.

With the $2 M \widehat{q}_{i}$ and $\widehat{p}_{i},(5.13)$ becomes

$$
\begin{aligned}
\partial_{t} f & +\sum_{i=1}^{M}\left\{\nabla_{\widehat{q}_{i}} \cdot\left(\alpha \widehat{q}^{\widehat{q}_{i}} f\right)+\nabla_{\widehat{p}_{i}} \cdot\left(\alpha^{\widehat{p}_{i}} f\right)\right\} \\
& -\frac{1}{2} \sum_{i, j=1}^{M} \sum_{\mu, \nu=1}^{d}\left\{\partial_{\widehat{q}_{i \mu}} \partial_{\widehat{q}_{j \nu}}\left(\gamma_{\mu \nu}^{\widehat{q}_{i} \widehat{q}_{j}} f\right)+2 \partial_{\widehat{q}_{i \mu}} \partial_{\widehat{p}_{j \nu}}\left(\gamma_{\mu \nu}^{\widehat{q}_{i} \widehat{p}_{j}} f\right)+\partial_{\widehat{p}_{i \mu}} \partial_{\widehat{p}_{j \nu}}\left(\gamma_{\mu \nu}^{\widehat{p}_{i} \widehat{p}_{j}} f\right)\right\}=0,
\end{aligned}
$$

where

$$
\begin{aligned}
\alpha^{\widehat{q}_{i}} & =\lim _{\Delta t \rightarrow 0} \frac{1}{\Delta t}\left\langle\widehat{q}_{i}(t+\Delta t)-\widehat{q}_{i}(t)\right\rangle \\
\alpha^{\widehat{p}_{i}} & =\lim _{\Delta t \rightarrow 0} \frac{1}{\Delta t}\left\langle\widehat{p}_{i}(t+\Delta t)-\widehat{p}_{i}(t)\right\rangle \\
\gamma^{\widehat{q}_{i} \widehat{q}_{j}} & =\lim _{\Delta t \rightarrow 0} \frac{1}{\Delta t}\left\langle\left(\widehat{q}_{i}(t+\Delta t)-\widehat{q}_{i}(t)\right) \otimes\left(\widehat{q}_{j}(t+\Delta t)-\widehat{q}_{j}(t)\right)\right\rangle \\
\gamma^{\widehat{q}_{i} \widehat{p}_{j}} & =\lim _{\Delta t \rightarrow 0} \frac{1}{\Delta t}\left\langle\left(\widehat{q}_{i}(t+\Delta t)-\widehat{q}_{i}(t)\right) \otimes\left(\widehat{p}_{j}(t+\Delta t)-\widehat{p}_{j}(t)\right)\right\rangle \\
\gamma^{\widehat{p}_{i} \widehat{p}_{j}} & =\lim _{\Delta t \rightarrow 0} \frac{1}{\Delta t}\left\langle\left(\widehat{p}_{i}(t+\Delta t)-\widehat{p}_{i}(t)\right) \otimes\left(\widehat{p}_{j}(t+\Delta t)-\widehat{p}_{j}(t)\right)\right\rangle .
\end{aligned}
$$

Here " $\langle\ldots\rangle "$ indicates an average over the ensemble of the $\mathscr{F}_{i}$, with $\widehat{q}(t)$ and $\widehat{p}(t)$ taken as fixed. Equations (5.16) imply that

$$
\begin{aligned}
& \widehat{q}_{i}(t+\Delta t)=\widehat{q}_{i}(t)+\Delta t \frac{1}{m} \widehat{p}_{i}(t)+O\left(\Delta t^{2}\right) \\
& \widehat{p}_{i}(t+\Delta t)=\widehat{p}_{i}(t)+\Delta t\left(-\nabla_{\widehat{q}_{i}} \widehat{\phi}(\widehat{q}(t))-\sum_{j=1}^{M} A^{i j}(\widehat{q}(t)) \widehat{p}_{j}(t)\right)+\int_{t}^{t+\Delta t} d s \mathscr{F}_{i}(s)+O\left(\Delta t^{2}\right),
\end{aligned}
$$

which can be used to evaluate the $\alpha$ 's and $\gamma$ 's via the same method as in the one-dimensional example above, to yield:

$$
\begin{aligned}
\alpha^{\widehat{q}_{i}} & =\frac{1}{m} \widehat{p}_{i} & \alpha^{\widehat{p}_{i}} & =-\nabla_{\widehat{q}_{i}} \widehat{\phi}-\sum_{j=1}^{M} A^{i j} \widehat{p}_{j} \\
\gamma^{\widehat{q}_{i} \widehat{q}_{j}} & =0 & \gamma^{\widehat{q}_{i} \widehat{p}_{j}} & =0
\end{aligned}
$$

and

$$
\gamma^{\widehat{p}_{i} \widehat{p}_{j}}=\lim _{\Delta t \rightarrow 0} \frac{1}{\Delta t} \int_{t}^{t+\Delta t} d s \int_{t}^{t+\Delta t} d s^{\prime}\left\langle\mathscr{F}_{i}(s) \otimes \mathscr{F}_{j}\left(s^{\prime}\right)\right\rangle .
$$

The most general condition that the $\mathscr{F}_{i}$ must satisfy in order for this last quantity to exist and be nonzero is

$$
\left\langle\mathscr{F}_{i}(s) \otimes \mathscr{F}_{j}\left(s^{\prime}\right)\right\rangle=B^{i j}(\widehat{q}(s), \widehat{p}(s), s) \delta\left(s-s^{\prime}\right),
$$

in which case

$$
\gamma^{\widehat{p}_{i} \widehat{p}_{j}}=B^{i j}(\widehat{q}, \widehat{p}, t)
$$


Here the $B^{i j}$ are a family of $d \times d$ matrices. We may additionally note that, since there are no explicitly time-dependent forces in the full system, the form of the reduced system's coefficients should be time-translationally invariant. Therefore, we can eliminate the explicit time dependence of the $B^{i j}$, so $B^{i j}=B^{i j}(\widehat{q}, \widehat{p})$. The expressions we have derived for the $\alpha$ 's and $\gamma$ 's allow us to write the Fokker-Planck equation (5.17) as

$\partial_{t} f=-\sum_{i=1}^{M}\left\{\frac{1}{m} \widehat{p}_{i} \cdot \nabla_{\widehat{q}_{i}} f-\nabla_{\widehat{p}_{i}} \cdot\left[\left(\nabla_{\widehat{q}_{i}} \widehat{\phi}+\sum_{j=1}^{M} A^{i j} \widehat{p}_{j}\right) f\right]\right\}+\frac{1}{2} \sum_{i, j=1}^{M} \sum_{\mu, \nu=1}^{M} \partial_{\widehat{p}_{i \mu}} \partial_{\widehat{p}_{j \nu}}\left(B_{\mu \nu}^{i j} f\right)$.

The distribution of the coordinates of the full system is assumed to come to equilibrium at the canonical probability density (2.24), which implies that the marginal distribution of the resolved variables will equilibrate to the renormalized canonical distribution $\hat{f}_{c}(\widehat{q}, \widehat{p})$, which is defined by equation (4.49). Thus, $\hat{f}_{c}$ must be a stationary solution of (5.19). This fact allows us to derive more properties of the matrices $B^{i j}$. If we eliminate the $\partial_{t} f$ term in $(5.19)$, set $f=\hat{f}_{c}$, and simplify the resulting expression, we arrive at

$$
\begin{aligned}
\hat{f}_{c}\left[-\sum_{i=1}^{M} \operatorname{Tr}\left\{A^{i i}\right.\right. & \left.-\frac{1}{2 m k_{B} T} B^{i i}\right\}+\frac{1}{m k_{B} T} \sum_{i, j=1}^{M} \widehat{p}_{i} \cdot\left\{\left(A^{i j}-\frac{1}{2 m k_{B} T} B^{i j}\right) \widehat{p}_{j}\right\} \\
& \left.-\frac{1}{2} \sum_{i, j=1}^{M} \sum_{\mu, \nu=1}^{M}\left\{\left(\partial_{\widehat{p}_{i \mu}} \partial_{\widehat{p}_{j \nu}} B_{\mu \nu}^{i j}\right)-\frac{1}{m k_{B} T}\left(\widehat{p}_{i \mu} \partial_{\widehat{p}_{j \nu}}+\widehat{p}_{j \nu} \partial_{\widehat{p}_{i \mu}}\right) B_{\mu \nu}^{i j}\right\}\right]=0,
\end{aligned}
$$

which is a necessary and sufficient condition for $\hat{f}_{c}$ to be a stationary state of (5.19). (Here $\operatorname{Tr}\{\ldots\}$ indicates the trace of a matrix.)

In order to simplify (5.20) somewhat, we make the additional assumption that the random forces $\mathscr{F}_{i}$ are independent of the resolved momenta $\widehat{p}_{i}$. This is a very common assumption in the literature, and can be justified as follows: The higher the momentum of a given resolved particle, the greater the degree of resistance it will feel from the surrounding "sea" of unresolved particles, due to a larger number of interparticle collisions. However, this net effect has already been accounted for by our approximation to the memory term (5.9), and the random forces $\mathscr{F}_{i}$ represent fluctuations from the mean contribution of these interactions. Such fluctuations result from factors such as the variations in local density and average velocity of the unresolved particles, which would depend weakly, if at all, on the momenta of the resolved particles.

We define a set of matrices $D^{i j}$ by the relation

$$
B^{i j}=2 m k_{B} T A^{i j}+D^{i j} .
$$

Equation (5.21), along with our assumption that the forces $\mathscr{F}_{i}$, and hence the matrices $B^{i j}$, are $\widehat{p}$-independent, allows us to write $(5.20)$ as

$$
\sum_{i=1}^{M} \operatorname{Tr}\left\{D^{i i}\right\}-\frac{1}{m k_{B} T} \sum_{i, j=1}^{M} \widehat{p}_{i} \cdot D^{i j} \widehat{p}_{j}=0
$$


which must be true for any choice of the $\widehat{p}_{i}$. This can be used to derive some conditions on the $D^{i j}$. If we choose to set $\widehat{p}_{i}=0$ for all $i$, then (5.22) becomes

$$
\sum_{i=1}^{M} \operatorname{Tr}\left\{D^{i i}\right\}=0 .
$$

However, if we instead choose $\widehat{p}_{i}=\widehat{p}_{k} \delta_{i k}$, for arbitrary $k$ and $\widehat{p}_{k}$, then (5.22), in combination with (5.23), reduces to

$$
\widehat{p}_{k} \cdot D^{k k} \widehat{p}_{k}=0 \quad \forall k, \widehat{p}_{k},
$$

which implies that $D^{k k}$ is skew-symmetric:

$$
\left(D^{k k}\right)^{T}=-D^{k k}
$$

Finally, if we let $\widehat{p}_{i}=\widehat{p}_{k} \delta_{i k}+\widehat{p}_{\ell} \delta_{i \ell}$, where $k \neq \ell$, then (5.22) becomes

$$
\begin{aligned}
0 & =\underbrace{\widehat{p}_{k} \cdot D^{k k} \widehat{p}_{k}}_{=0 \text { by }(5.24)}+\widehat{p}_{k} \cdot D^{k \ell} \widehat{p}_{\ell}+\widehat{p}_{\ell} \cdot D^{\ell k} \widehat{p}_{k}+\underbrace{\widehat{p}_{\ell} \cdot D^{\ell \ell} \widehat{p}_{\ell}}_{=0 \text { by }(5.24)} \\
& =\widehat{p}_{\ell} \cdot\left(\left(D^{k \ell}\right)^{T}+D^{\ell k}\right) \widehat{p}_{k},
\end{aligned}
$$

which can be true for all $\widehat{p}_{k}$ and $\widehat{p}_{\ell}$ if and only if

$$
\left(D^{k \ell}\right)^{T}=-D^{\ell k} \quad \text { for } \quad k \neq \ell .
$$

Note that equations $(5.21),(5.24)$, and (5.25) are equivalent to the statement

$$
\mathcal{B}=2 m k_{B} T \mathcal{A}+\mathcal{D} \quad \text { where } \quad \mathcal{D}^{T}=-\mathcal{D} .
$$

Here $\mathcal{A}$ is as defined in (5.11), and $\mathcal{B}$ and $\mathcal{D}$ are similarly defined as the $M d \times M d$ block matrices of the $B^{i j}$ and $D^{i j}$, respectively. From (5.26), and the fact that $\mathcal{A}$ is positive semi-definite, it follows that $\mathcal{B}$ is positive semi-definite, as well. This implies that the $B^{i j}$ satisfy a similar theorem to Theorem 5.1.

Equation (5.21) or, equivalently, (5.26), is an example of a fluctuation-dissipation relation. Along with the supplementary conditions (5.24) and (5.25), they describe most general constraints that the $B^{i j}$ must satisfy in order to ensure that $\hat{f}_{c}(\widehat{q}, \widehat{p})$ is a stationary state of the Fokker-Planck equation (5.19). Note, however, that we have not yet arrived at an approximate form for the forces $\mathscr{F}_{i}$. The expression (5.18) serves to define the covariances of the $\mathscr{F}_{i}$ in terms of the $B^{i j}$, but does not tell us how to evaluate the forces themselves in an actual simulation. An expression for the $\mathscr{F}_{i}$ derived from an approximation to the friction matrices $A^{i j}$ will be given in a later section.

\subsection{Relaxation to Equilibrium}

In Section 5.2 we have shown, given our approximation (5.9) to the memory term, and given certain conditions on the covariances of the random forces, that the renormalized canonical distribution (RCD) $\hat{f}_{c}$ is a stationary state of the Fokker-Planck equation (5.19). However, we have yet to show that this state is likely to be reached from some given initial 
distribution, which is necessary if the linear friction approximation is to be useful. It is the goal of this section to prove that the distribution of the $\widehat{q}_{i}$ and $\widehat{p}_{i}$ will equilibrate to $\hat{f}_{c}$ from any initial state. (Some of the notation here, as well as parts of the proof of Theorem 5.5, have been adapted from [40].)

If the $B^{i j}$ are momentum-independent, then the Fokker-Planck equation can be written as

$$
\partial_{t} f=-\left(L_{C}+L_{D}\right) f
$$

where

$$
L_{C} \equiv \sum_{i=1}^{M}\left\{\frac{1}{m} \widehat{p}_{i} \cdot \nabla_{\widehat{q}_{i}}-\left(\nabla_{\widehat{q}_{i}} \widehat{\phi}\right) \cdot \nabla_{\widehat{p}_{i}}\right\}
$$

is the Liouville operator associated with the FOOP system (4.47), and

$$
L_{D} \equiv-\sum_{i, j=1}^{M} \nabla_{\widehat{p}_{i}} \cdot\left\{A^{i j} \widehat{p}_{j}+\frac{1}{2} B^{i j} \nabla_{\widehat{p}_{j}}\right\}
$$

is the dissipative Liouville operator which incorporates the effects of the friction and random forcing.

In general, our probability density function $f(\widehat{q}, \widehat{p}, t)$ can be thought of as a function of $\widehat{q}$ and $\widehat{p}$, parameterized by the time $t$. We will assume that $\widehat{p}_{i} \in \mathbb{R}^{d}$, and $\widehat{q}_{i} \in \Omega \subseteq \mathbb{R}^{d}$ for all $1 \leq i \leq M$. Furthermore, we assume boundary conditions (BC) such that $f \rightarrow 0$ as $\left|\widehat{p}_{i}\right| \rightarrow \infty$, and such that $f$ is either periodic in $\widehat{q}_{i}$, or $f \rightarrow 0$ as $\widehat{q}_{i} \rightarrow \partial \Omega$, where $\partial \Omega$ is the boundary of $\Omega$.

We now define $\mathcal{Q}$ as the set of all functions $g$ of $\widehat{q}$ and $\widehat{p}$ that satisfy

$$
\begin{aligned}
& \text { - } \int d \widehat{q} \int d \widehat{p} g(\widehat{q}, \widehat{p})=1 \\
& \text { - } g(\widehat{q}, \widehat{p}) \geq 0 \quad \forall \widehat{q}, \widehat{p} .
\end{aligned}
$$

$\mathcal{Q}$ is the set of all probability density functions on the $\widehat{q}$ and $\widehat{p}$. Note that $\mathcal{Q}$ is a subset of $L^{1}\left(\Omega^{M} \times \mathbb{R}^{M d}\right)$, and that $\hat{f}_{c} \in \mathcal{Q}$. The significance of $\mathcal{Q}$ is illustrated by the following theorem:

Theorem 5.2 The set $\mathcal{Q}$ is invariant under the flow described by the Fokker-Planck equation (5.27).

Proof: Let $f(\widehat{q}, \widehat{p}, t)$ be a solution to $(5.27)$, with initial condition $f(\widehat{q}, \widehat{p}, 0)=f_{0}(\widehat{q}, \widehat{p})$. In order to prove this theorem, it suffices to show that

(1) If $f_{0}(\widehat{q}, \widehat{p})$ satisfies (5.30), then so does $f(\widehat{q}, \widehat{p}, t)$ for all $t$.

(2) If $f_{0}(\widehat{q}, \widehat{p})$ satisfies (5.31), then so does $f(\widehat{q}, \widehat{p}, t)$ for all $t$.

We begin by noting that the Fokker-Planck equation can be written somewhat more compactly in terms of the matrices $\mathcal{A}$ and $\mathcal{B}$ as

$$
\partial_{t} f=-\left(\frac{1}{m} \widehat{p} \cdot \nabla_{\widehat{q}}-\left(\nabla_{\widehat{q}} \widehat{\phi}\right) \cdot \nabla_{\widehat{p}}\right) f+\nabla_{\widehat{p}} \cdot\left(\mathcal{A} \widehat{p}+\frac{1}{2} \mathcal{B} \nabla_{\widehat{p}}\right) f
$$

where $\nabla_{\widehat{q}}$ and $\nabla_{\widehat{p}}$ are the $M d$-dimensional gradients in $\widehat{q}$ and $\widehat{p}$, respectively. 
We now define the functional

$$
\mathcal{N}[f] \equiv \int d \widehat{q} \int d \widehat{p} f(\widehat{q}, \widehat{p}, t)
$$

Then

$$
\begin{aligned}
\partial_{t} \mathcal{N}[f] & =\int d \widehat{q} \int d \widehat{p} \partial_{t} f(\widehat{q}, \widehat{p}, t) \\
& =-\int d \widehat{q} \int d \widehat{p}\left[\nabla_{\widehat{q}} \cdot\left(\frac{1}{m} \widehat{p} f\right)-\nabla_{\widehat{p}} \cdot\left(\nabla_{\widehat{q}} \widehat{\phi} f+\mathcal{A} \widehat{p} f+\frac{1}{2} \mathcal{B} \nabla_{\widehat{p}} f\right)\right] \\
& =0
\end{aligned}
$$

where the last equality follows from the boundary conditions we've assumed. This proves (1).

The proof of (2) is more involved. Suppose there exists some time $t^{*}$ such that $f(\widehat{q}, \widehat{p}, t) \geq 0$ for all $0 \leq t \leq t^{*}$. We do not rule out the possibility that $t^{*}=0$. We now imagine that $f\left(\widehat{q}^{*}, \widehat{p}^{*}, t^{*}\right)=0$ for some $\widehat{q}^{*}$ and $\widehat{p}^{*}$. Rearranging (5.32) somewhat allows us to write the time derivative of $f$ at $\left(\widehat{q}^{*}, \widehat{p}^{*}, t^{*}\right)$ as

$$
\begin{aligned}
\left.\partial_{t} f\right|_{\left(\widehat{q}^{*}, \widehat{p}^{*}, t^{*}\right)}= & -\left.\frac{1}{m} \widehat{p} \cdot \nabla_{\widehat{q}} f\right|_{\left(\widehat{q}^{*}, \widehat{p}^{*}, t^{*}\right)}+\left.\left(\nabla_{\widehat{q}} \widehat{\phi}\right) \cdot \nabla_{\widehat{p}} f\right|_{\left(\widehat{q}^{*}, \widehat{p}^{*}, t^{*}\right)} \\
& +\left.\operatorname{Tr}\{\mathcal{A}\} f\right|_{\left(\widehat{q}^{*}, \widehat{p}^{*}, t^{*}\right)}+\left.\left(\nabla_{\widehat{p}} f\right) \cdot \mathcal{A} \widehat{p}\right|_{\left(\widehat{q}^{*}, \widehat{p}^{*}, t^{*}\right)}+\left.\frac{1}{2} \operatorname{Tr}\left\{\mathcal{B}\left(\nabla_{\widehat{p}} \otimes \nabla_{\widehat{p}} f\right)\right\}\right|_{\left(\widehat{q}^{*}, \widehat{p}^{*}, t^{*}\right)} .
\end{aligned}
$$

The fact that $f\left(\widehat{q}^{*}, \widehat{p}^{*}, t^{*}\right)=0$ means that the third term on the righthand side above is zero. Furthermore, since by assumption $f\left(\widehat{q}, \widehat{p}, t^{*}\right) \geq f\left(\widehat{q}^{*}, \widehat{p}^{*}, t^{*}\right)$ for all $\widehat{q}$ and $\widehat{p}$, it follows that

$$
\left.\nabla_{\widehat{q}} f\right|_{\left(\widehat{q}^{*}, \widehat{p}^{*}, t^{*}\right)}=0 \quad \text { and }\left.\quad \nabla_{\widehat{p}} f\right|_{\left(\widehat{q}^{*}, \widehat{p}^{*}, t^{*}\right)}=0 .
$$

This implies that the first, second, and fourth terms are zero as well, after which we are left with

$$
\left.\partial_{t} f\right|_{\left(\widehat{q}^{*}, \widehat{p}^{*}, t^{*}\right)}=\left.\frac{1}{2} \operatorname{Tr}\left\{\mathcal{B}\left(\nabla_{\widehat{p}} \otimes \nabla_{\widehat{p}} f\right)\right\}\right|_{\left(\widehat{q}^{*}, \widehat{p}^{*}, t^{*}\right)} .
$$

The matrix $\left(\nabla_{\widehat{p}} \otimes \nabla_{\widehat{p}} f\right)$ of second derivatives of $f$ must be positive semi-definite at $\left(\widehat{q}^{*}, \widehat{p}^{*}, t^{*}\right)$, since $f$ is either flat or a minimum in each of the $2 M d$ coordinate directions at that point. This then implies that

$$
\left.\partial_{t} f\right|_{\left(\widehat{q}^{*}, \widehat{p}^{*}, t^{*}\right)} \geq 0
$$

because $\mathcal{B}$ is positive semi-definite, and the trace of the product of two positive semi-definite matrices is always greater than or equal to zero.

The above argument shows that any $f$ which is greater than or equal to zero everywhere for $0 \geq t \geq t^{*}$ cannot pass through zero under the flow generated by (5.32) at any point $\left(\widehat{q}^{*}, \widehat{p}^{*}, t^{*}\right)$, since $f$ is nondecreasing when $f=0$. This proves $(2)$.

Now that we have proven that solutions to the Fokker-Planck equation that start out in $\mathcal{Q}$ stay in $\mathcal{Q}$ for all time, we can proceed to analyze the flow in $\mathcal{Q}$ generated by (5.27). We define an energy functional

$$
E[f] \equiv \int d \widehat{q} \int d \widehat{p} \mathscr{H}(\widehat{q}, \widehat{p}) f(\widehat{q}, \widehat{p})
$$


where $\mathscr{H}=\sum_{i=1}^{M}\left|\widehat{p}_{i}\right| / 2 m+\widehat{\phi}(\widehat{q})$ is the renormalized Hamiltonian defined in Chapter 4, as well as an entropy functional

$$
S[f] \equiv-k_{B} \int d \widehat{q} \int d \widehat{p} \log (f(\widehat{q}, \widehat{p})) f(\widehat{q}, \widehat{p}),
$$

and a Helmholtz free energy functional

$$
F[f] \equiv E[f]-T S[f]=\int d \widehat{q} \int d \widehat{p}\left(\mathscr{H}(\widehat{q}, \widehat{p})+k_{B} T \log (f(\widehat{q}, \widehat{p}))\right) f(\widehat{q}, \widehat{p}) .
$$

We will eventually be using $F[f]$ as a Lyapunov functional to show that all solutions equilibrate to $\hat{f}_{c}$. The first step is to show that $\hat{f}_{c}$ minimizes $F[f]$.

Theorem $5.3 F\left[\hat{f}_{c}\right] \leq F[f]$ for any $f \in \mathcal{Q}$, with equality if and only if $f=\hat{f}_{c}$.

Proof: Any element $f \in \mathcal{Q}$ can be written as

$$
f(\widehat{q}, \widehat{p})=\hat{f}_{c}(\widehat{q}, \widehat{p})+\delta f(\widehat{q}, \widehat{p}),
$$

for some $\delta f$. By (5.31), $\delta f(\widehat{q}, \widehat{p}) \geq-\hat{f}_{c}(\widehat{q}, \widehat{p})$ for all $\widehat{q}$ and $\widehat{p}$. Furthermore, (5.30) implies that

$$
\int d \widehat{q} \int d \widehat{p} \delta f(\widehat{q}, \widehat{p})=0 .
$$

We then have:

$$
\begin{aligned}
& F[f]= F\left[\hat{f}_{c}+\delta f\right] \\
&= \int d \widehat{q} \int d \widehat{p}\left(\mathscr{H}+k_{B} T \log \left(\hat{f}_{c}+\delta f\right)\right)\left(\hat{f}_{c}+\delta f\right) \\
&= \underbrace{\int d \widehat{q} \int d \widehat{p}\left(\mathscr{H}+k_{B} T \log \left(\hat{f}_{c}\right)\right) \hat{f}_{c}}_{=F\left[\hat{f}_{c}\right]}+\underbrace{\int d \widehat{q} \int d \widehat{p} \delta f\left(\mathscr{H}+k_{B} T \log \left(\hat{f}_{c}\right)\right)}_{=-k_{B} T \log (Z) \int d \widehat{q} \int d \widehat{p} \delta f(\widehat{q}, \widehat{p})=0} \\
& \quad+k_{B} T \int d \widehat{q} \int d \widehat{p} \log \left(1+\frac{\delta f}{\hat{f}_{c}}\right)\left(\hat{f}_{c}+\delta f\right) \\
&=F\left[\hat{f}_{c}\right]+k_{B} T \int d \widehat{q} \int d \widehat{p} \hat{f}_{c}\left(1+\frac{\delta f}{\hat{f}_{c}}\right) \log \left(1+\frac{\delta f}{\hat{f}_{c}}\right) .
\end{aligned}
$$

The fact that $(1+x) \log (1+x) \geq x$ for all $x \geq-1$, with equality if and only if $x=0$, tells us that

$$
\left(1+\frac{\delta f}{\hat{f}_{c}}\right) \log \left(1+\frac{\delta f}{\hat{f}_{c}}\right) \geq \frac{\delta f}{\hat{f}_{c}}
$$

in which case

$$
\begin{aligned}
F[f] & \geq F\left[\hat{f}_{c}\right]+k_{B} T \int d \widehat{q} \int d \widehat{p} \hat{f}_{c} \frac{\delta f}{\hat{f}_{c}} \\
& \geq F\left[\hat{f}_{c}\right]+k_{B} T \int d \widehat{q} \int d \widehat{p} \delta f \\
& \geq F\left[\hat{f}_{c}\right],
\end{aligned}
$$

where the equality holds only when $\delta f=0$, i.e. when $f=\hat{f}_{c}$.

The next step is to show that $\hat{f}_{c}$ is the only minimum of $F$. 
Theorem 5.4 Let $f^{*}$ be an extremum of $F$ in $\mathcal{Q}$. Then $f^{*}=\hat{f}_{c}$.

Proof: We define the functional

$$
G[f]=F[f]+\lambda\left(\int d \widehat{q} \int d \widehat{p} f(\widehat{q}, \widehat{p})-1\right),
$$

where $\lambda$ is a Lagrange multiplier, and the purpose of the second term is to enforce the constraint (5.30). The functional derivative of $G$ at $f^{*}$ must be zero if $f^{*}$ is to be an extremum:

$$
\left.\frac{\delta}{\delta f} G[f]\right|_{f=f^{*}}=\mathscr{H}+k_{B} T\left(1+\log \left(f^{*}\right)\right)+\lambda=0,
$$

or

$$
f^{*}=\exp \left(-1-\frac{\lambda}{k_{B} T}\right) e^{-\beta \mathscr{H}} .
$$

Using the normalization condition (5.30) allows us to solve for $\lambda$, to yield $f^{*}=e^{-\beta \mathscr{H}} / Z=\hat{f}_{c}$.

Theorems 5.3 and 5.4 prove that $\hat{f}_{c}$ is the unique, global minimum of $F$. Our next goal is to show that $F$ will approach this minimum monotonically in time. In order to accomplish this, we define $\mathcal{Q}_{K} \subset \mathcal{Q}$ as the set of all $f \in \mathcal{Q}$ that have the form

$$
f(\widehat{q}, \widehat{p})=\frac{g(\widehat{q})}{\left(2 \pi m k_{B} T\right)^{M d / 2}} \exp \left(-\beta \sum_{i=1}^{M} \frac{\left|\widehat{p}_{i}\right|^{2}}{2 m}\right) .
$$

Clearly, $g$ must satisfy $g \geq 0$ and $\int d \widehat{q} g(\widehat{q})=1$. The following theorem then holds:

Theorem 5.5 If $\mathcal{A}$ is strictly positive definite, and $f \in \mathcal{Q}$ is a solution to the Fokker-Planck equation (5.27) or, equivalently, (5.32), then

$$
\partial_{t} F[f] \leq 0,
$$

with equality if and only if $f \in \mathcal{Q}_{K}$.

\section{Proof:}

$$
\begin{aligned}
\partial_{t} F[f]= & \int d \widehat{q} \int d \widehat{p}\left(\mathscr{H}+k_{B} T \log (f)\right) \partial_{t} f+k_{B} T \underbrace{\int d \widehat{q} \int d \widehat{p} \partial_{t} f}_{=0 \text { by Theorem } 5.2} \\
= & -\int d \widehat{q} \int d \widehat{p}\left(\mathscr{H}+k_{B} T \log (f)\right) L_{C} f-\int d \widehat{q} \int d \widehat{p}\left(\mathscr{H}+k_{B} T \log (f)\right) L_{D} f \\
= & \int d \widehat{q} \int d \widehat{p}(\underbrace{L_{C} \mathscr{H}}_{=0} f+\underbrace{k_{B} T L_{C} f}_{\text {yields } 0 \text { by BC }}) \\
& +\int d \widehat{q} \int d \widehat{p}\left(\mathscr{H}+k_{B} T \log (f)\right) \nabla_{\widehat{p}} \cdot\left\{\left(\mathcal{A} \widehat{p}+\frac{1}{2} \mathcal{B} \nabla_{\widehat{p}}\right) f\right\},
\end{aligned}
$$

where in the third equality we have used the fact that $L_{C}$ is a first-order differential operator to integrate by parts. If we also integrate the last expression above by parts in $\widehat{p}$, and use the fact that $\nabla_{\widehat{p}} f=f \nabla_{\widehat{p}} \log (f)$, we arrive at

$$
\partial_{t} F[f]=-\int d \widehat{q} \int d \widehat{p} \frac{1}{m} f\left(\widehat{p}+m k_{B} T \nabla_{\widehat{p}} \log (f)\right) \cdot\left(\mathcal{A} \widehat{p}+\frac{1}{2} \mathcal{B} \nabla_{\widehat{p}} \log (f)\right) .
$$


Substituting in the expression (5.26) for $\mathcal{B}$ yields:

$$
\begin{aligned}
\partial_{t} F[f]=-\int d \widehat{q} \int d \widehat{p} & \frac{1}{m} f\left(\widehat{p}+m k_{B} T \nabla_{\widehat{p}} \log (f)\right) \cdot \mathcal{A}\left(\widehat{p}+m k_{B} T \nabla_{\widehat{p}} \log (f)\right) \\
& -\int d \widehat{q} \int d \widehat{p} \frac{1}{2 m} f\left(\widehat{p}+m k_{B} T \nabla_{\widehat{p}} \log (f)\right) \cdot \mathcal{D} \nabla_{\widehat{p}} \log (f) .
\end{aligned}
$$

The second term on the righthand side above can be manipulated as follows:

$$
\begin{aligned}
& \int d \widehat{q} \int d \widehat{p} \frac{1}{2 m} f\left(\widehat{p}+m k_{B} T \nabla_{\widehat{p}} \log (f)\right) \cdot \mathcal{D} \nabla_{\widehat{p}} \log (f) \\
&=\int d \widehat{p} \frac{1}{2 m} f(\widehat{p} \cdot \mathcal{D} \nabla_{\widehat{p}} \log (f)+m k_{B} T \underbrace{\nabla_{\widehat{p}} \log (f) \cdot \mathcal{D} \nabla_{\widehat{p}} \log (f)}_{=0 \text { since } \mathcal{D} \text { is skew-symmetric }}) .
\end{aligned}
$$

If we again make use of $f \nabla_{\widehat{p}} \log (f)=\nabla_{\widehat{p}} f$, and integrate by parts, this expression becomes $\int d \widehat{q} \int d \widehat{p} \operatorname{Tr}\{\mathcal{D}\} f=0$, and the original expression simplifies to

$$
\begin{aligned}
\partial_{t} F[f] & =-\int d \widehat{q} \int d \widehat{p} \frac{1}{m} f\left(\widehat{p}+m k_{B} T \nabla_{\widehat{p}} \log (f)\right) \cdot \mathcal{A}\left(\widehat{p}+m k_{B} T \nabla_{\widehat{p}} \log (f)\right) \\
& \leq 0
\end{aligned}
$$

where the last step follows from the fact that $\mathcal{A}$ is positive definite. Equality is obtained if and only if

$$
\widehat{p}+m k_{B} T \nabla_{\widehat{p}} \log (f)=0,
$$

which implies that $f \in \mathcal{Q}_{K}$.

Theorems 5.3, 5.4, and 5.5 above allow us to finally accomplish the goal of this section:

Theorem 5.6 If $f(\widehat{q}, \widehat{p}, t)$ is a solution of the Fokker-Planck equation (5.32), and $\mathcal{A}$ is strictly positive definite, then $f \rightarrow \hat{f}_{c}$ asymptotically as $t \rightarrow \infty$.

Proof: According to Theorem 5.5, if $\mathcal{A}$ is positive definite, then $\partial_{t} F[f]<0$ for $f \notin \mathcal{Q}_{K}$. Theorems 5.3 and 5.4 tell us that $\hat{f}_{c}$ is the only minimum of $F$. This means that $f$ will approach $\hat{f}_{c}$ monotonically, until it either reaches it, or enters the set $\mathcal{Q}_{K}$. Therefore, all that remains to establish global equilibration to $\hat{f}_{c}$ is to show that $\hat{f}_{c}$ is the only fixed point of the Fokker-Planck equation in $\mathcal{Q}_{K}$. This can be accomplished as follows: Let $f^{*} \in \mathcal{Q}_{K}$ be written as in (5.37). We can substitute this expression into (5.32), to derive an equation for the evolution of $g$, the $\widehat{q}$-dependent part of $f^{*}$. Performing this substitution and simplifying the resulting expression yields

$$
\partial_{t} g=-\frac{1}{m} \widehat{p} \cdot\left\{\nabla_{\widehat{q}} g+\frac{1}{k_{B} T}\left(\nabla_{\widehat{q}} \widehat{\phi}\right) g\right\},
$$

which must be zero for all $\widehat{p}$ in order for $f^{*}$ to be a stationary state. However, this can only be realized by assuming that

$$
\nabla_{\widehat{q}} g+\frac{1}{k_{B} T}\left(\nabla_{\widehat{q}} \widehat{\phi}\right) g=0
$$

which implies that $f^{*}=\hat{f}_{c}$. Any other form for $g$ will necessarily cause $f^{*}$ to leave the set $\mathcal{Q}_{K}$, and continue its approach to $\hat{f}_{c}$. Since $\hat{f}_{c}$ is the only stationary point in $\mathcal{Q}_{K}$, and any point off of $\mathcal{Q}_{K}$ approaches $\hat{f}_{c}$ monotonically, the theorem is established. 
Theorem 5.6 shows us that our reduced system (5.16) will equilibrate to the renormalized canonical distribution from any initial state. It does not tell us the time scale of the equilibration. The amount of time necessary to obtain an adequate approximation to the RCD from some arbitrary, presumably highly non-canonical, state must be determined experimentally by simulating the system. One important thing to note is that any numerical scheme designed to integrate the equations of motion (5.16) will not require an additional thermostat, such as the Nosé-Hoover thermostat of Chapter 4, to maintain its temperature. The balance between the memory and random forcing terms implied by the fluctuationdissipation relation (5.26) will accomplish that for us.

\subsection{Approximating the Friction Matrices and Random Forces}

The expression (5.8) for the friction matrices $A^{i j}$ is not particularly useful as it is written, since it depends in general on all $M$ of the $\widehat{x}_{i}$, and involves a projection which we have no way of evaluating exactly. We will therefore make several approximations to (5.8) which are closely related to the approximation made in Chapter 4, where we neglected many-particle effects in order to derive an expression for the renormalized potential $\widehat{\phi}$.

We start with the off-diagonal sub-matrices of $\mathcal{A}$, the $A^{i j}$ with $i \neq j$. Using the fact that

$$
\phi(x)=\sum_{i<j}^{N} U\left(\left|x_{i}-x_{j}\right|\right) \equiv \sum_{i<j}^{N} U_{i j},
$$

as well as the approximation (4.48)

$$
\widehat{\phi}(\widehat{x}) \approx \sum_{i<j}^{M} \widehat{U}\left(\left|\widehat{x}_{i}-\widehat{x}_{j}\right|\right) \equiv \sum_{i<j}^{M} \widehat{U}_{i j},
$$

allows us to write $A^{i j}(\widehat{x})$ as

$$
A^{i j}(\widehat{x}) \approx \frac{\tau}{m}\left[\left(\nabla_{\widehat{x}_{i}} \otimes \nabla_{\widehat{x}_{j}}\right)\left(U_{i j}-\widehat{U}_{i j}\right)\right] \quad i \neq j .
$$

If we now define $\delta \widehat{U}=\widehat{U}-U$ to be the amount by which the renormalized potential differs from the bare potential, then we arrive at

$$
A^{i j}(\widehat{x}) \approx \frac{\tau}{m}\left(\nabla_{\widehat{x}_{i}} \otimes \nabla_{\widehat{x}_{i}}\right) \delta \widehat{U}_{i j} \quad i \neq j
$$

where we have used the fact that $\nabla_{\widehat{x}_{j}} U_{i j}=-\nabla_{\widehat{x}_{i}} U_{i j}$ and $\nabla_{\widehat{x}_{j}} \widehat{U}_{i j}=-\nabla_{\widehat{x}_{i}} \widehat{U}_{i j}$. This expression can be cast in a more intuitive form by applying the useful relation

$$
\left(\nabla_{\widehat{x}_{i}} \otimes \nabla_{\widehat{x}_{i}}\right) \Theta\left(\left|\widehat{x}_{i}-\widehat{x}_{j}\right|\right)=\Theta^{\prime \prime}\left(\left|\widehat{x}_{i}-\widehat{x}_{j}\right|\right) P^{i j}+\frac{\Theta^{\prime}\left(\left|\widehat{x}_{i}-\widehat{x}_{j}\right|\right)}{\left|\widehat{x}_{i}-\widehat{x}_{j}\right|} Q^{i j},
$$

where $\Theta$ is an arbitrary function,

$$
P^{i j} \equiv \frac{\left(\widehat{x}_{i}-\widehat{x}_{j}\right) \otimes\left(\widehat{x}_{i}-\widehat{x}_{j}\right)}{\left|\widehat{x}_{i}-\widehat{x}_{j}\right|^{2}}
$$

is the $d \times d$ spatial projection matrix, which, when applied to any vector, picks out the component parrallel to $\widehat{x}_{i}-\widehat{x}_{j}$, and $Q^{i j}=I-P^{i j}$ is its complement. Note that $\left(P^{i j}\right)^{2}=P^{i j}$, 
$\left(Q^{i j}\right)^{2}=Q^{i j}$, and $P^{i j} Q^{i j}=Q^{i j} P^{i j}=0$. Note also that the $P^{i j}$ and $Q^{i j}$ are symmetric and positive semi-definite. Combining (5.38) and (5.39) yields

$$
A^{i j}(\widehat{x}) \approx \frac{\tau}{m}\left(\delta \widehat{U}_{i j}^{\prime \prime} P^{i j}+\frac{\delta \widehat{U}_{i j}^{\prime}}{\left|\widehat{x}_{i j}\right|} Q^{i j}\right) \quad i \neq j .
$$

The $i=j$ case is somewhat more involved, but the same principles may be applied. The expression for $A^{i i}$ can be divided into two parts as follows:

$$
\begin{aligned}
A^{i i}(\widehat{x}) & =\frac{\tau}{m}\left[\mathbb{P}\left\{\left(\nabla_{\widehat{x}_{i}} \otimes \nabla_{\widehat{x}_{i}}\right) \phi(x)\right\}-\left(\nabla_{\widehat{x}_{i}} \otimes \nabla_{\widehat{x}_{i}}\right) \widehat{\phi}(\widehat{x})\right] \\
& \approx \frac{\tau}{m}\left[\sum_{j \neq i}^{N} \mathbb{P}\left\{\left(\nabla_{\widehat{x}_{i}} \otimes \nabla_{\widehat{x}_{i}}\right) U_{i j}\right\}-\sum_{j \neq i}^{M}\left(\nabla_{\widehat{x}_{i}} \otimes \nabla_{\widehat{x}_{i}}\right) \widehat{U}_{i j}\right] \\
& =\frac{\tau}{m}\left[\sum_{j \neq i}^{M}\left(\nabla_{\widehat{x}_{i}} \otimes \nabla_{\widehat{x}_{i}}\right) U_{i j}+\sum_{j=M+1}^{N} \mathbb{P}\left\{\left(\nabla_{\widehat{x}_{i}} \otimes \nabla_{\widehat{x}_{i}}\right) U_{i j}\right\}-\sum_{j \neq i}^{M}\left(\nabla_{\widehat{x}_{i}} \otimes \nabla_{\widehat{x}_{i}}\right) \widehat{U}_{i j}\right] \\
& =\frac{\tau}{m}\left[(N-M) \mathbb{P}\left\{\left(\nabla_{\widehat{x}_{i}} \otimes \nabla_{\widehat{x}_{i}}\right) U_{i M+1}\right\}-\sum_{j \neq i}^{M}\left(\nabla_{\widehat{x}_{i}} \otimes \nabla_{\widehat{x}_{i}}\right) \delta \widehat{U}_{i j}\right]
\end{aligned}
$$

where in the second line we have again approximated $\widehat{\phi}$ by its pair term, and in the fourth line we have made use of the indistinguishability of the unresolved particles.

The remaining projection in (5.42) can be written as

$$
\begin{aligned}
(N-M) \mathbb{P}\{( & \left.\left(\nabla_{\widehat{x}_{i}} \otimes \nabla_{\widehat{x}_{i}}\right) U_{i M+1}\right\} \\
& =(N-M) \frac{\int d \widetilde{x}\left(\nabla_{\widehat{x}_{i}} \otimes \nabla_{\widehat{x}_{i}}\right) U_{i M+1} e^{-\beta \phi}}{\int d \widetilde{x} e^{-\beta \phi}} \\
& =(N-M) \frac{\int d \widetilde{x}_{1}\left(\nabla_{\widehat{x}_{i}} \otimes \nabla_{\widehat{x}_{i}}\right) U\left(\left|\widehat{x}_{i}-\widetilde{x}_{1}\right|\right) \int d \widetilde{x}_{2} \ldots d \widetilde{x}_{N-M} e^{-\beta \phi}}{\int d \widetilde{x} e^{-\beta \phi}} .
\end{aligned}
$$

This expression can be simplified somewhat by using the relations

$$
\int d \widetilde{x} e^{-\beta \phi}=\widetilde{\rho}^{M} \frac{(N-M) !}{N !} g^{(M)}(\widehat{x}, \widetilde{\rho}, T) \int d x e^{-\beta \phi}
$$

and

$$
\int d \widetilde{x}_{2} \ldots d \widetilde{x}_{N-M} e^{-\beta \phi}=\widetilde{\rho}^{M+1} \frac{(N-M-1) !}{N !} g^{(M+1)}\left(\widehat{x}, \widetilde{x}_{1}, \widetilde{\rho}, T\right) \int d x e^{-\beta \phi},
$$

which follow from the definition of the $M$-particle and $M+1$-particle distribution functions. We then have:

$$
(N-M) \mathbb{P}\left\{\left(\nabla_{\widehat{x}_{i}} \otimes \nabla_{\widehat{x}_{i}}\right) U_{i M+1}\right\}=\widetilde{\rho} \frac{\int d \widetilde{x}_{1}\left(\nabla_{\widehat{x}_{i}} \otimes \nabla_{\widehat{x}_{i}}\right) U\left(\left|\widehat{x}_{i}-\widetilde{x}_{1}\right|\right) g^{(M+1)}\left(\widehat{x}, \widetilde{x}_{1}, \widetilde{\rho}, T\right)}{g^{(M)}(\widehat{x}, \widetilde{\rho}, T)} .
$$


Equation (5.43) is an exact expression for the projection term in (5.42). However, it is not useful as written, since we do not have access to the full $M$-particle and $M+1$-particle distribution functions. We may write these distribution functions in terms of the potentials of mean force described in Chapter 4:

$$
g^{(M)}(\widehat{x})=\exp \left[-\beta \psi^{(M)}(\widehat{x})\right] \quad \text { and } \quad g^{(M+1)}\left(\widehat{x}, \widetilde{x}_{1}\right)=\exp \left[-\beta \psi^{(M+1)}\left(\widehat{x}, \widetilde{x}_{1}\right)\right],
$$

where we have omitted the arguments $\widetilde{\rho}$ and $T$ for clarity. Here

$$
\psi^{(M)}(\widehat{x})=\sum_{i<j}^{M} \widehat{U}\left(\left|\widehat{x}_{i}-\widehat{x}_{j}\right|\right)+\sum_{i<j<k}^{M} \widehat{U}_{3}\left(\left|\widehat{x}_{i}-\widehat{x}_{j}\right|,\left|\widehat{x}_{j}-\widehat{x}_{k}\right|,\left|\widehat{x}_{k}-\widehat{x}_{i}\right|\right)+\cdots
$$

is the same as the renormalized potential, to within constants [23]. We may also write $\psi^{(M+1)}$ as:

$$
\psi^{(M+1)}\left(\widehat{x}, \widetilde{x}_{1}\right)=\psi^{(M)}(\widehat{x})+\sum_{i=1}^{M} \widehat{U}\left(\left|\widehat{x}_{i}-\widetilde{x}_{1}\right|\right)+\sum_{i<j}^{M} \widehat{U}_{3}\left(\left|\widehat{x}_{i}-\widetilde{x}_{1}\right|,\left|\widehat{x}_{j}-\widetilde{x}_{1}\right|,\left|\widehat{x}_{i}-\widehat{x}_{j}\right|\right)+\cdots
$$

If we now ignore the three-particle and higher interactions in (5.46), and use the fact that $g^{(2)}=\exp [-\beta \widehat{U}]$, then we arrive at

$$
g^{(M+1)}\left(\widehat{x}, \widetilde{x}_{1}\right) \approx g^{(M)}(\widehat{x}) \prod_{j=1}^{M} g^{(2)}\left(\left|\widehat{x}_{j}-\widetilde{x}_{1}\right|\right) .
$$

This approximation simplifies (5.43) to:

$$
(N-M) \mathbb{P}\left\{\left(\nabla_{\widehat{x}_{i}} \otimes \nabla_{\widehat{x}_{i}}\right) U_{i M+1}\right\} \approx \widetilde{\rho} \int d \widetilde{x}_{1}\left(\nabla_{\widehat{x}_{i}} \otimes \nabla_{\widehat{x}_{i}}\right) U\left(\left|\widehat{x}_{i}-\widetilde{x}_{1}\right|\right) \prod_{j=1}^{M} g^{(2)}\left(\left|\widehat{x}_{j}-\widetilde{x}_{1}\right|\right) \text {. }
$$

The expression (5.47) still involves an intractable integral that depends on all $M$ of the $\widehat{x}_{i}$. In order to remedy this, we express the product of radial distribution functions in (5.47) as a Mayer-like expansion, analogous to the one used in Chapter 4 to derive the renormalized potential:

$$
\begin{aligned}
& \prod_{j=1}^{M} g^{(2)}\left(\left|\widehat{x}_{j}-\widetilde{x}_{1}\right|\right) \\
& \quad=g^{(2)}\left(\left|\widehat{x}_{i}-\widetilde{x}_{1}\right|\right) \prod_{j \neq i}^{M} g^{(2)}\left(\left|\widehat{x}_{j}-\widetilde{x}_{1}\right|\right) \\
& \quad=g^{(2)}\left(\left|\widehat{x}_{i}-\widetilde{x}_{1}\right|\right)\left\{1+\sum_{j \neq i}^{M}\left(g^{(2)}\left(\left|\widehat{x}_{j}-\widetilde{x}_{1}\right|\right)-1\right)\right. \\
& \left.\quad+\sum_{j \neq i}^{M} \sum_{k \neq i, j}^{M}\left(g^{(2)}\left(\left|\widehat{x}_{j}-\widetilde{x}_{1}\right|\right)-1\right)\left(g^{(2)}\left(\left|\widehat{x}_{k}-\widetilde{x}_{1}\right|\right)-1\right)+\cdots\right\} .
\end{aligned}
$$


The advantage of this is that whereas $g^{(2)}(r) \rightarrow 1$ as $r \rightarrow \infty$, and is thus long-ranged, $\left(g^{(2)}(r)-1\right) \rightarrow 0$ as $r \rightarrow \infty$, with a length scale equal to that of the renormalized potential. The first term in braces above represents an effect that depends only on the coordinates of the single resolved particle $\widehat{x}_{i}$, the second term yields an interaction between two resolved particles $\left(\widehat{x}_{i}\right.$ and $\left.\widehat{x}_{j}\right)$, the third term generates a three-particle interaction $\left(\widehat{x}_{i}, \widehat{x}_{j}\right.$, and $\left.\widehat{x}_{k}\right)$, and so on. In keeping with our previous approximations, we will neglect all but the first two of these terms, which yields

$$
\begin{aligned}
(N-M) \mathbb{P}\left\{\left(\nabla_{\widehat{x}_{i}} \otimes \nabla_{\widehat{x}_{i}}\right) U_{i M+1}\right\} & \\
\approx \widetilde{\rho} & \int d \widetilde{x}_{1}\left(\nabla_{\widehat{x}_{i}} \otimes \nabla_{\widehat{x}_{i}}\right) U\left(\left|\widehat{x}_{i}-\widetilde{x}_{1}\right|\right) g^{(2)}\left(\left|\widehat{x}_{i}-\widetilde{x}_{1}\right|\right) \\
& \quad+\sum_{j \neq i}^{M} \widetilde{\rho} \int d \widetilde{x}_{1}\left(\nabla_{\widehat{x}_{i}} \otimes \nabla_{\widehat{x}_{i}}\right) U\left(\left|\widehat{x}_{i}-\widetilde{x}_{1}\right|\right) g^{(2)}\left(\left|\widehat{x}_{i}-\widetilde{x}_{1}\right|\right)\left(g^{(2)}\left(\left|\widehat{x}_{j}-\widetilde{x}_{1}\right|\right)-1\right) .
\end{aligned}
$$

We will consider the two terms in the truncated expansion above one at a time. The first can be simplified by realizing that the presence of the factor $\left(\nabla_{\widehat{x}_{i}} \otimes \nabla_{\widehat{x}_{i}}\right) U\left(\left|\widehat{x}_{i}-\widetilde{x}_{1}\right|\right)$ in the integrand ensures that it is short-ranged, i.e. it rapidly approaches zero as the integration variable $\widetilde{x}_{1}$ moves away from $\widehat{x}_{i}$. This implies that, unless $\widehat{x}_{i}$ is very close to the edge of the domain (a boundary effect we ignore, as usual), we can shift the origin of the integral to eliminate the $\widehat{x}_{i}$ dependence:

$$
\int d \widetilde{x}_{1}\left(\nabla_{\widehat{x}_{i}} \otimes \nabla_{\widehat{x}_{i}}\right) U\left(\left|\widehat{x}_{i}-\widetilde{x}_{1}\right|\right) g^{(2)}\left(\left|\widehat{x}_{i}-\widetilde{x}_{1}\right|\right)=\int d y\left(\nabla_{y} \otimes \nabla_{y}\right) U(|y|) g^{(2)}(|y|) .
$$

Since there is no preferred direction in the above integral, symmetry implies that the matrix it generates must be isotropic, i.e.

$$
\int d y\left(\nabla_{y} \otimes \nabla_{y}\right) U(|y|) g^{(2)}(|y|)=\Gamma I
$$

where $I$ is the identity matrix and

$$
\Gamma=\Gamma(\widetilde{\rho}, T) \equiv \frac{1}{3} \int d y \triangle U(|y|) g^{(2)}(|y|, \widetilde{\rho}, T) .
$$

(Here " $y$ " is a dummy variable of integration.)

The integral in (5.50) depends sensitively on values of $g^{(2)}(r)$ at small $r$, which are difficult to ascertain numerically, due to the hard-core repulsion present in most interparticle potentials. It is therefore more accurate to evaluate $\Gamma$ directly, by running a MD simulation of the full system at density $\widetilde{\rho}$ and temperature $T$, and using the equivalent expression

$$
\Gamma(\widetilde{\rho}, T)=\frac{2}{3 \widetilde{\rho} N}\left\langle\sum_{i<j}^{N} \triangle U\left(\left|x_{i}-x_{j}\right|\right)\right\rangle_{C},
$$

which follows from the definition of $g^{(2)}$. A graph of $\Gamma$ as a function of density, as calculated via this method for the Lennard-Jones potential at reduced temperature $T^{*}=1.6$, is shown in Figure 5.1. Note that $\Gamma$ is a relatively large, positive number for all densities. The discontinuity near $\rho^{*}=1.0$ is due to the fluid-solid phase transition. 


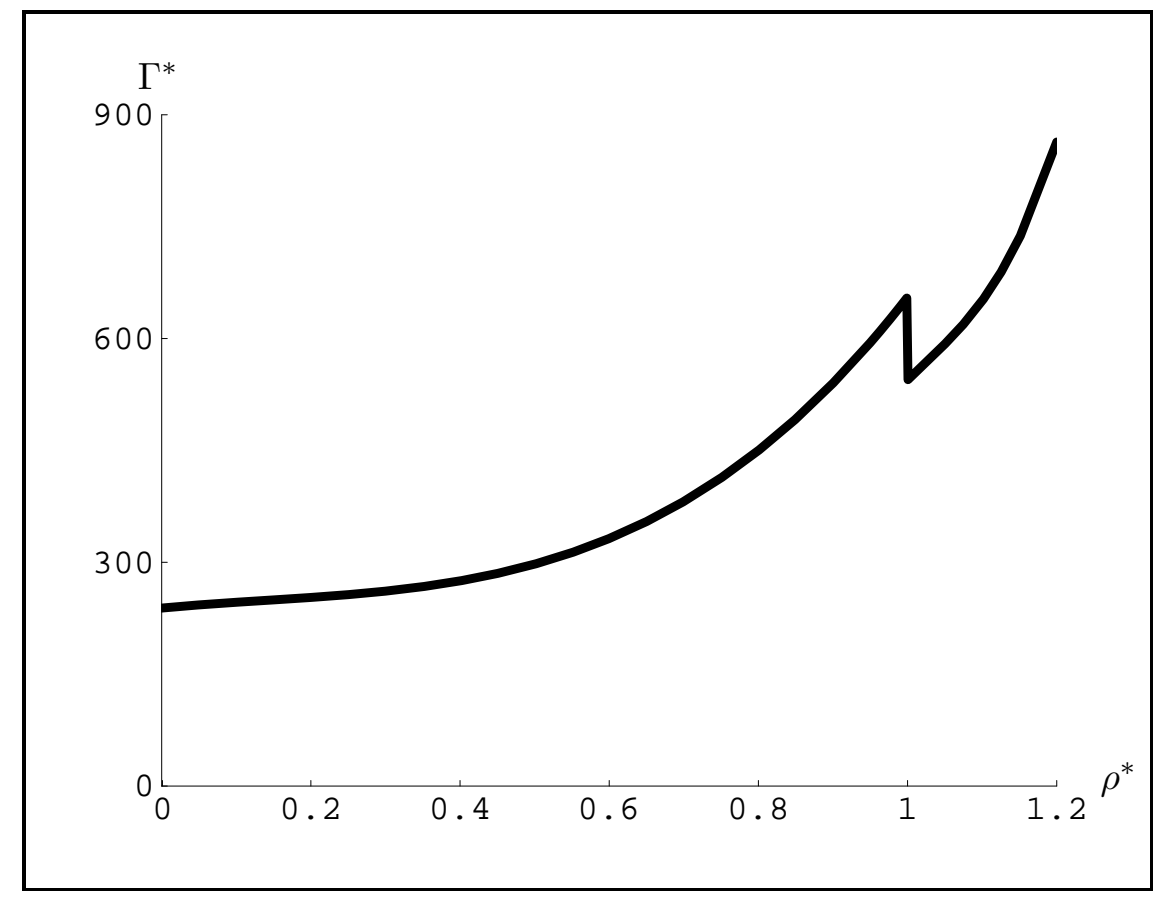

Figure 5.1: $\Gamma^{*}$ as a function of $\rho^{*}$ for the Lennard-Jones system at $T^{*}=1.6$.

We now consider the integral in the second term of (5.48):

$$
\int d \widetilde{x}_{1}\left(\nabla_{\widehat{x}_{i}} \otimes \nabla_{\widehat{x}_{i}}\right) U\left(\left|\widehat{x}_{i}-\widetilde{x}_{1}\right|\right) g^{(2)}\left(\left|\widehat{x}_{i}-\widetilde{x}_{1}\right|\right)\left(g^{(2)}\left(\left|\widehat{x}_{j}-\widetilde{x}_{1}\right|\right)-1\right) .
$$

This cannot be simplified completely using spatial isotropy, as we did to arrive at (5.49), because there is now a preferred direction, namely the vector $\widehat{x}_{i j}=\widehat{x}_{i}-\widehat{x}_{j}$ joining the two resolved coordinates. However, cylindrical symmetry around $\widehat{x}_{i j}$ tells us two things: first, that the matrix generated by (5.52) can depend only on the magnitude of $\widehat{x}_{i j}$, second, that it can be divided into a longitudinal part that acts on components of vectors parrallel to $\widehat{x}_{i j}$, as well as a transverse part that acts on components perpendicular to $\widehat{x}_{i j}$.

We begin by shifting the origin of (5.52) to $\widehat{x}_{i}$, and rotate the coordinates of the integrand so that $\widehat{x}_{i j}$ points along the positive $d^{\text {th }}$ axis. If we further define $r \equiv\left|\widehat{x}_{i j}\right|$, then (5.52) becomes

$$
S_{i j}^{-1} \int d y\left(\nabla_{y} \otimes \nabla_{y}\right) U(|y|) g^{(2)}(|y|)\left(g^{(2)}\left(\left|y-r e_{d}\right|\right)-1\right) S_{i j},
$$

where $S_{i j}$ is any $d \times d$ rotation matrix that brings $\widehat{x}_{i j}$ parallel to the $d^{\text {th }}$ axis. In this basis,

$$
\begin{aligned}
\int d y\left(\nabla_{y} \otimes \nabla_{y}\right) U(|y|) g^{(2)}(|y|) & \left(g^{(2)}\left(\left|y-r e_{d}\right|\right)-1\right) \\
& =\operatorname{Diag}\left(\xi^{\perp}(r), \xi^{\perp}(r), \ldots, \xi^{\perp}(r), \xi^{\|}(r)\right),
\end{aligned}
$$

where

$$
\xi^{\|}(r) \equiv \int d y \frac{\partial^{2}}{\partial y_{d}^{2}} U(|y|) g^{(2)}(|y|)\left(g^{(2)}\left(\left|y-r e_{d}\right|\right)-1\right)
$$


(a)

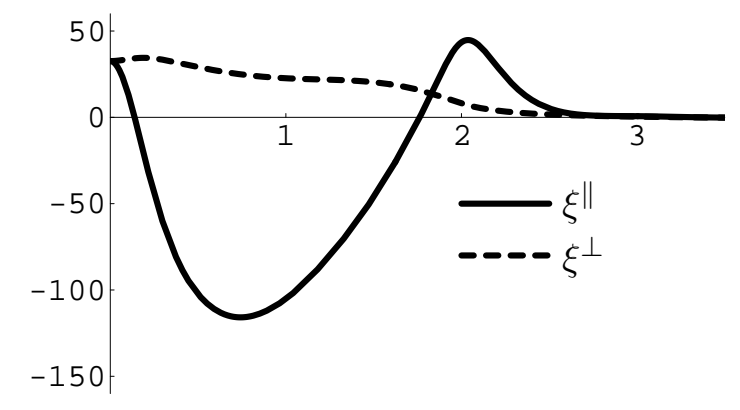

(b)

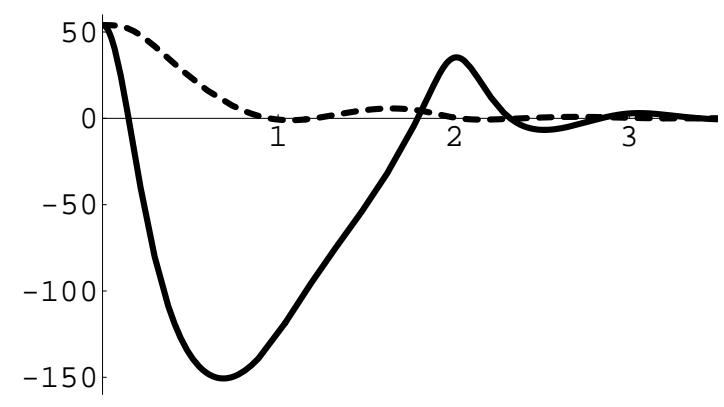

(c)

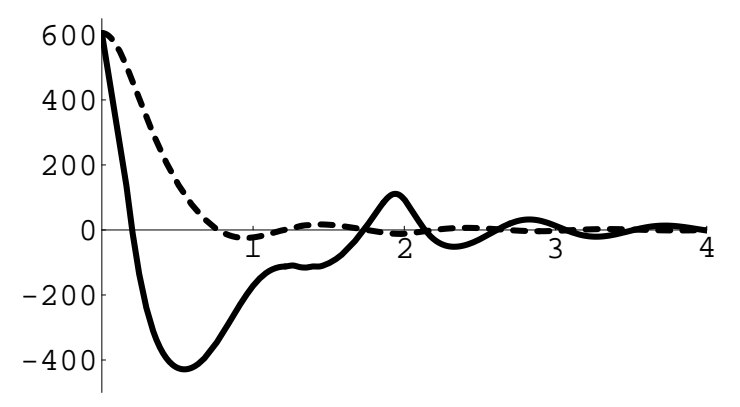

Figure 5.2: The functions $\xi^{\|}$and $\xi^{\perp}$ for the Lennard-Jones potential at $T^{*}=1.6$, in reduced units. a) $\widetilde{\rho}^{*}=0.0657$ b) $\widetilde{\rho}^{*}=0.4148$ c) $\widetilde{\rho}^{*}=0.9625$.

and

$$
\xi^{\perp}(r) \equiv \int d y \frac{\partial^{2}}{\partial y_{1}^{2}} U(|y|) g^{(2)}(|y|)\left(g^{(2)}\left(\left|y-r e_{d}\right|\right)-1\right) .
$$

The shapes of the functions $\xi^{\|}$and $\xi^{\perp}$ depend on the state variables $\widetilde{\rho}$ and $T$. In $d=3$, $\xi^{\|}$and $\xi^{\perp}$ can be calculated most efficiently using the expressions

$$
\begin{aligned}
\xi^{\|}(r)=2 \pi \int_{0}^{\infty} d R \int_{-1}^{1} d \alpha\left(\left(1-\alpha^{2}\right) R U^{\prime}(R)+\right. & \left.\alpha^{2} R^{2} U^{\prime \prime}(R)\right) g^{(2)}(R) \\
& \times\left(g^{(r)}\left(\sqrt{R^{2}+r^{2}-2 R r \alpha}\right)-1\right) \\
\xi^{\perp}(r)=-\frac{1}{2} \xi^{\|}(r)+\pi \int_{0}^{\infty} d R \int_{-1}^{1} d \alpha R\left(2 U^{\prime}(R)+\right. & \left.R U^{\prime \prime}(R)\right) g^{(2)}(R) \\
& \times\left(g^{(r)}\left(\sqrt{R^{2}+r^{2}-2 R r \alpha}\right)-1\right)
\end{aligned}
$$

which follow by switching to spherical polar coordinates. Examples of $\xi^{\|}$and $\xi^{\perp}$ for the Lennard-Jones system at various values of $\widetilde{\rho}$ are shown in Figure 5.2.

It should be clear that equations (5.53) and (5.54) together are equivalent to

$$
\xi^{\|}(r) P^{i j}+\xi^{\perp}(r) Q^{i j}
$$


in which case our expression (5.48) for the projection becomes

$$
\begin{aligned}
(N-M) \mathbb{P} & \left\{\left(\nabla_{\widehat{x}_{i}} \otimes \nabla_{\widehat{x}_{i}}\right) U_{i M+1}\right\} \\
& \approx \widetilde{\rho}\left[\Gamma(\widetilde{\rho}, T) I+\sum_{j \neq i}^{M}\left(\xi^{\|}\left(\left|\widehat{x}_{i j}\right|\right) P^{i j}+\xi^{\perp}\left(\left|\widehat{x}_{i j}\right|\right) Q^{i j}\right)\right] .
\end{aligned}
$$

This, when combined with (5.42), yields the final approximate form for the matrices $A^{i i}$ :

$$
A^{i i}(\widehat{x}) \approx \frac{\tau}{m}\left[\widetilde{\rho} \Gamma I+\sum_{j \neq i}^{M}\left(\chi_{i j}^{(1)} I+\chi_{i j}^{(2)} P^{i j}\right)\right]
$$

where we have used the fact that $Q^{i j}=I-P^{i j}$, and defined two new functions for convenience,

$$
\begin{aligned}
& \chi_{i j}^{(1)} \equiv \widetilde{\rho} \xi_{i j}^{\perp}-\frac{\delta \widehat{U}_{i j}^{\prime}}{\left|\widehat{x}_{i j}\right|} \\
& \chi_{i j}^{(2)} \equiv \widetilde{\rho}\left(\xi_{i j}^{\|}-\xi_{i j}^{\perp}\right)+\frac{\delta \widehat{U}_{i j}^{\prime}}{\left|\widehat{x}_{i j}\right|}-\delta \widehat{U}_{i j}^{\prime \prime} .
\end{aligned}
$$

Examples of $\chi^{(1)}(r)$ and $\chi^{(2)}(r)$ for several densities are plotted in Figure 5.3. Note that these functions are shown only for $r^{*}>1$. This is because the hard-core repulsion of the Lennard-Jones potential makes $\delta \widehat{U}(r)$ difficult to determine accurately at small distances. This should not be a problem, however, because for $r^{*} \leq 1$ the interaction between any two resolved particles is dominated by the strong repulsive forces of the renormalized pair potential, and friction becomes less important. In any case, other than at very high resolved densities, such encounters should be extremely rare.

The form of our approximations (5.41) and (5.57) to the friction matrices $A^{i j}$ is highly suggestive. All of the pairwise functions (i.e. $\delta \widehat{U}^{\prime \prime}(r), \delta \widehat{U}^{\prime}(r) / r, \chi^{(1)}(r)$, and $\left.\chi^{(2)}(r)\right)$ involved in determining the $A^{i j}$ go to zero at large separation. This implies that a resolved particle in isolation will feel an isotropic friction governed solely by the surrounding sea of unresolved particles, with

$$
A_{o}^{i j}=0 \quad(i \neq j) \quad \text { and } \quad A_{o}^{i i}=\frac{\tau}{m} \widetilde{\rho} \Gamma I,
$$

where we have defined the independent parts $A_{o}^{i j}$ of the friction matrices. The deviations from isotropy embodied in (5.41) and (5.57) are a result of the fact that the distribution of unresolved particles around any given resolved particle is altered by the proximity of other resolved particles.

We now turn to the evaluation of the random forces $\mathscr{F}_{i}(t)$. Equation (5.18) tells us that the $\mathscr{F}_{i}$ satisfy

$$
\left\langle\mathscr{F}_{i}(t) \otimes \mathscr{F}_{j}\left(t^{\prime}\right)\right\rangle=B^{i j} \delta\left(t-t^{\prime}\right)
$$

This can be written more concisely by defining the $M d$-dimensional vector $\mathcal{F}$, where $\mathcal{F}(t) \equiv$ $\left(\mathscr{F}_{1}(t), \ldots, \mathscr{F}_{M}(t)\right)$, in which case we have

$$
\left\langle\mathcal{F}(t) \otimes \mathcal{F}\left(t^{\prime}\right)\right\rangle=\mathcal{B} \delta\left(t-t^{\prime}\right) .
$$



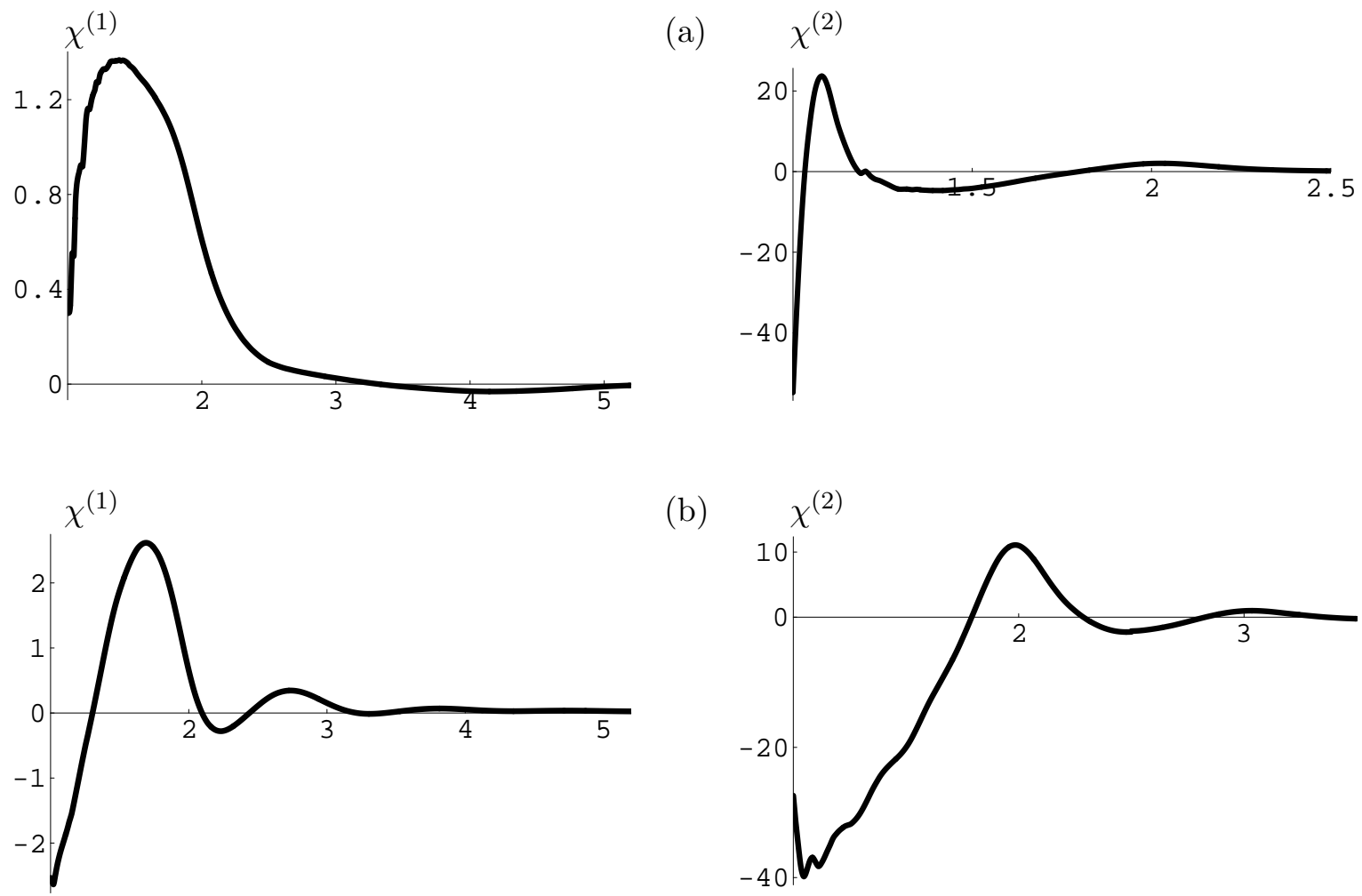

(b)
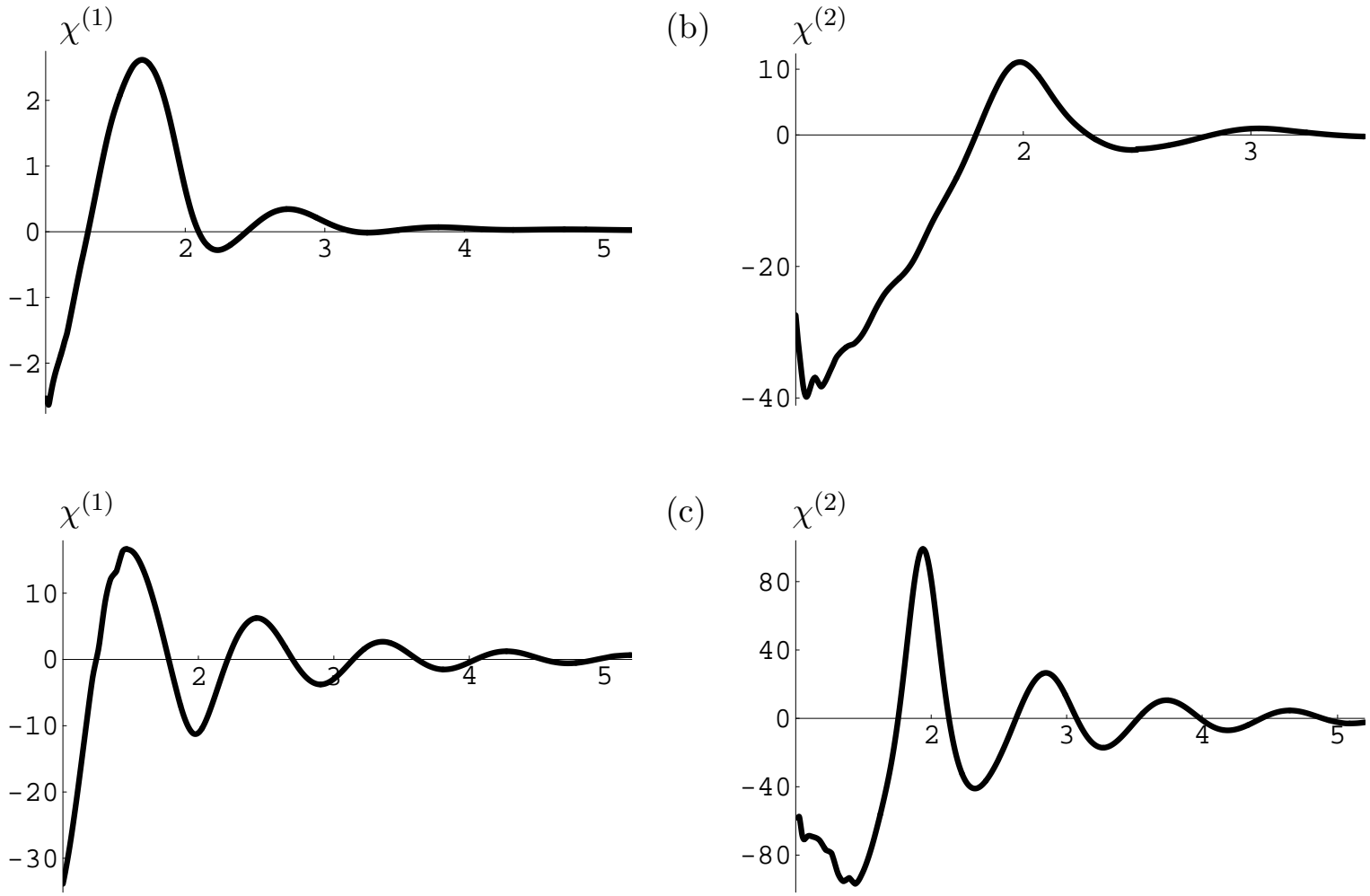

(c)

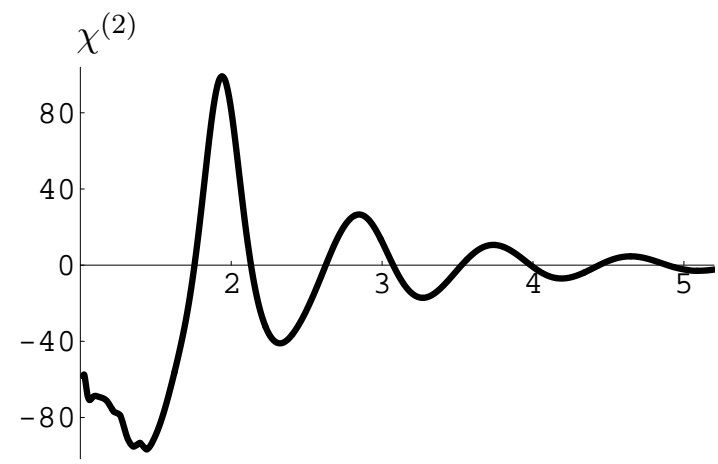

Figure 5.3: The functions $\chi^{(1)}$ and $\chi^{(2)}$ for the Lennard-Jones potential at $T^{*}=1.6$, in reduced units. a) $\widetilde{\rho}^{*}=0.0657$ b) $\widetilde{\rho}^{*}=0.4148$ c) $\widetilde{\rho}^{*}=0.9625$. 
The fact that $\mathcal{F}$ is $\delta$-correlated implies that it must be some linear combination of white noise processes. The most general way (5.59) can be realized is thus

$$
\mathcal{F}(t)=\mathcal{G} \mathcal{W}(t)
$$

Here $\mathcal{G}$ is a real, $M d \times M^{\prime}$ matrix, with $M^{\prime} \geq M d$, and $\mathcal{W}(t)$ is an $M^{\prime}$-dimensional vector of standard white noise processes, with components $\mathcal{W}_{\mu}(t)$ that satisfy

$$
\left\langle\mathcal{W}_{\mu}(t)\right\rangle=0 \quad \text { and } \quad\left\langle\mathcal{W}_{\mu}(t) \mathcal{W}_{\nu}\left(t^{\prime}\right)\right\rangle=\delta_{\mu \nu} \delta\left(t-t^{\prime}\right) .
$$

In order for (5.59) to hold, we must also have

$$
\mathcal{G G}^{T}=\mathcal{B}=2 m k_{B} T \mathcal{A}+\mathcal{D},
$$

where we have used (5.26). The fact that $\mathcal{G G}^{T}$ is symmetric for all real matrices $\mathcal{G}$ immediately implies that the previously arbitrary skew-symmetric matrix $\mathcal{D}$ must be zero, since $\mathcal{A}$ is symmetric by construction, and (5.60) cannot hold otherwise.

We will restrict ourselves to solutions with $M^{\prime}=M d$, in which case $\mathcal{G}$ is a square matrix that must obey

$$
\mathcal{G G}^{T}=2 m k_{B} T \mathcal{A}
$$

by (5.60). For the general case of $\mathcal{A}$ described by the approximations (5.41) and (5.57), there is no analytical solution to (5.61) for all configurations $\widehat{x}$ of the resolved particles. However, if these approximations preserve the positive-definiteness of $\mathcal{A}$, then we may calculate $\mathcal{G}$ numerically at each timestep as a lower-triangular real matrix via a Cholesky decomposition [51], and, in principle, determine the random forces $\mathscr{F}_{i}$. Unfortunately, in practice $\mathcal{A}$ is an extremely large $M d \times M d$ matrix, where, for the numerical experiments we are conducting, $1,500 \leq M d \leq 12,000$. For these matrix sizes, performing a single Cholesky decomposition requires at least several minutes, and so this method is untenable in a simulation of several thousand timesteps. Some additional approximation must therefore be made.

We choose to ignore the contributions to $\mathcal{A}$ from the off-diagonal sub-matrices $A^{i j}$ with $i \neq j$. This is equivalent to assuming that the frictional force felt by each resolved particle depends only on its own momentum, and not on the momenta of any other surrounding resolved particles. We can justify this numerically by looking at the magnitude of the contribution to the frictional force from the off-diagonal elements of $\mathcal{A}$. The frictional force on particle $i$ can be divided into a diagonal part and an off-diagonal part as follows:

$$
\begin{aligned}
-\sum_{j=1}^{M} A^{i j}(\widehat{q}) \widehat{p}_{j} & =-A^{i i}(\widehat{q}) \widehat{p}_{i}-\sum_{j \neq i}^{M} A^{i j}(\widehat{q}) \widehat{p}_{j} \\
& =-\frac{\tau}{m}\left(\theta_{i}^{D}(\widehat{q}, \widehat{p})+\theta_{i}^{O D}(\widehat{q}, \widehat{p})\right),
\end{aligned}
$$

where, according to (5.41) and (5.57),

$$
\begin{aligned}
\theta_{i}^{D}(\widehat{q}, \widehat{p}) & =\left[\tilde{\rho} \Gamma I+\sum_{j \neq i}^{M}\left(\chi_{i j}^{(1)} I+\chi_{i j}^{(2)} P^{i j}\right)\right] \widehat{p}_{i}, \\
\theta_{i}^{O D}(\widehat{q}, \widehat{p}) & =\sum_{j \neq i}^{M}\left[\frac{\delta \widehat{U}_{i j}^{\prime}}{\left|\widehat{q}_{i j}\right|} I+\left(\delta \widehat{U}_{i j}^{\prime \prime}-\frac{\delta \widehat{U}_{i j}^{\prime}}{\left|\widehat{q}_{i j}\right|}\right) P^{i j}\right] \widehat{p}_{j} .
\end{aligned}
$$




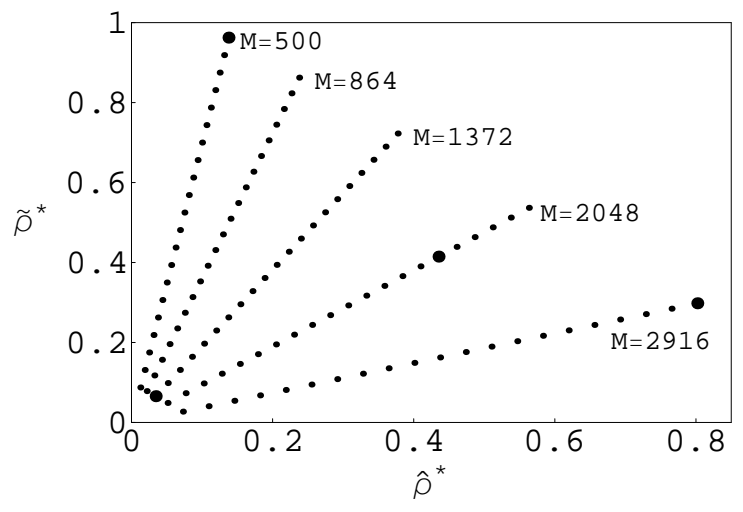

Figure 5.4: The set of $\left(\widehat{\rho}^{*}, \widetilde{\rho}^{*}\right)$ pairs considered in this thesis, in reduced units. Bold points represent those states described in Figures 5.5 and 5.6.

If ignoring the off-diagonal contributions to the friction is to be a plausible approximation, we must have $\left|\theta_{i}^{D}\right|$ significantly greater than $\left|\theta_{i}^{O D}\right|$ for most probable configurations. The average magnitudes of these quantities in a typical simulation depend on the state variables $T$, $\widehat{\rho}$, and $\widetilde{\rho}$. All of the $(\widehat{\rho}, \widetilde{\rho})$ pairs we consider in this thesis are shown in Figure 5.4. In order to check the relative size of the $\theta_{i}^{D}$ and $\theta_{i}^{O D}$, we select several representative points from this graph (those shown in bold), and perform a thermostated run of the FOOP system described in Chapter 4, at temperature $T^{*}=1.6$, and with the corresponding resolved and unresolved densities. This can be used to generate configurations of the resolved particles that are drawn from the renormalized canonical ensemble. These are then used to evaluate the $\theta_{i}^{D}$ and $\theta_{i}^{O D}$ for all $1 \leq i \leq M$, and compare the distribution of their magnitudes. The results of such a calculation for the Lennard-Jones system are shown in Figure 5.5. We see that the typical values of $\left|\theta^{D}\right|$ are usually about 4 to 10 times larger than those of $\left|\theta^{O D}\right|$.

Under the above approximation, we can write the random force on particle $i$ as $\mathscr{F}_{i}(t)=$ $G^{i i} W^{i}(t)$, where $G^{i i}$ is a real, $d \times d$ matrix that satisfies $G^{i i}\left(G^{i i}\right)^{T}=2 m k_{B} T A^{i i}$, and $W^{i}(t)$ is a $d$-dimensional vector of white noise processes. In general, there is no analytical solution to this for all configurations of the resolved particles, so we will still be performing Cholesky decompositions to find the $G^{i i}$. However, our labor has been greatly reduced from the calculation of a single $M d \times M d$ matrix, to the much more tractable task of finding $M d \times d$ matrices, which is an $O(M)$ process that can be done quite rapidly.

All that remains in order to show that this can work is to prove that the diagonal submatrices $A^{i i}$ of $\mathcal{A}$ are themselves positive definite. Unfortunately, this is not the case in general. It is possible to construct configurations $\widehat{x}$ of the resolved particles such that one or more of the $A^{i i}$ is not positive definite. However, as we will see, these configurations have an extremely low probability under the renormalized canonical distribution, and are thus not likely to occur very frequently in an actual simulation.

A necessary and sufficient condition for a matrix to be positive definite is that all of its eigenvalues be positive. The properties of the matrices $A^{i i}$ depend on the state variables $\widetilde{\rho}$ and $T$. In addition, the set of probable configurations of the resolved particles depends on the resolved density $\widehat{\rho}$. We can generate a set of values for the matrices $A^{i i}$ by sampling configurations from the renormalized canonical ensemble via the method described above. This will give us a set of $M d \times d$ matrices, from which $M d$ eigenvalues can be calculated. 

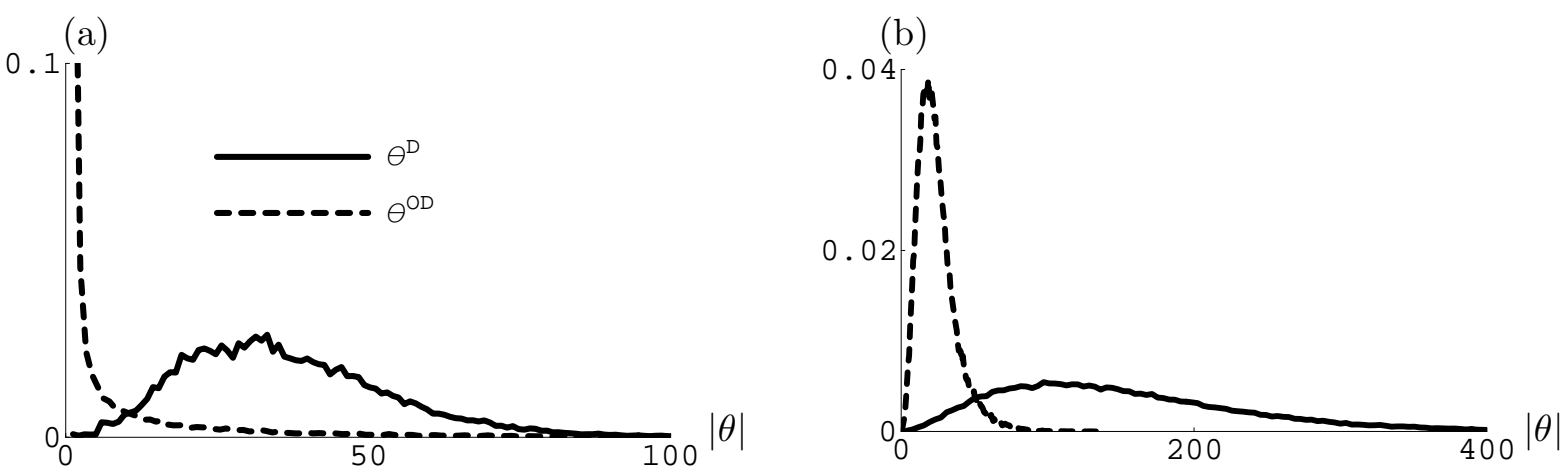

(c)

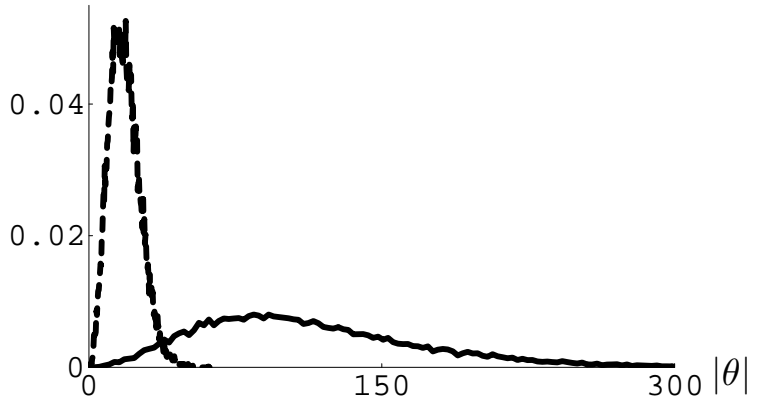

(d)

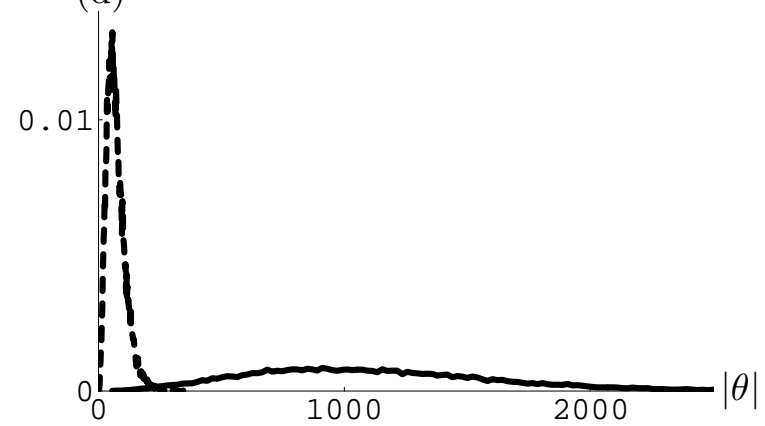

Figure 5.5: The probability distributions of $\left|\theta^{D}\right|$ and $\left|\theta^{O D}\right|$ for several density pairs, in reduced units. a) $\widehat{\rho}^{*}=0.0343, \widetilde{\rho}^{*}=0.0657$ b) $\widehat{\rho}^{*}=0.4352, \widetilde{\rho}^{*}=0.4148$ c) $\widehat{\rho}^{*}=0.8019$, $\widetilde{\rho}^{*}=0.2981$ d) $\widehat{\rho}^{*}=0.1375, \widetilde{\rho}^{*}=0.9625$. 

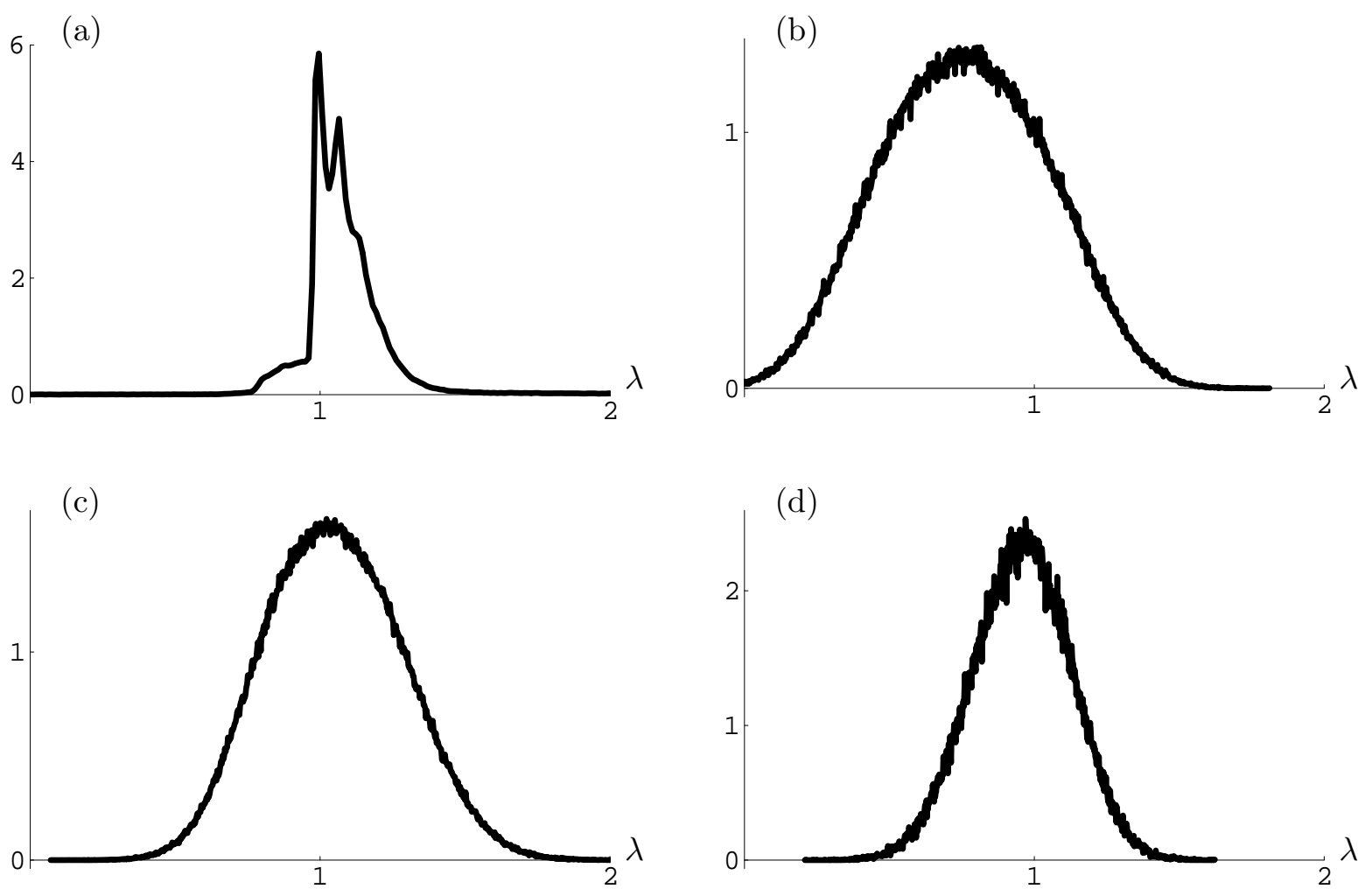

Figure 5.6: The probability distributions of $\lambda$, a typical eigenvalue of $A^{i i}$, for several density pairs, in reduced units and scaled by the corresponding value of $\tau^{*} \widetilde{\rho}^{*} \Gamma^{*}$. a) $\widehat{\rho}^{*}=0.0343$, $\widetilde{\rho}^{*}=0.0657$ b) $\widehat{\rho}^{*}=0.4352, \quad \widetilde{\rho}^{*}=0.4148$ c) $\widehat{\rho}^{*}=0.8019, \quad \widetilde{\rho}^{*}=0.2981$ d) $\widehat{\rho}^{*}=0.1375$, $\widetilde{\rho}^{*}=0.9625$.

We denote the $\mu^{\text {th }}$ eigenvalue of the $i^{\text {th }}$ matrix as $\lambda_{\mu}^{i}$, where $1 \leq i \leq M$ and $1 \leq \mu \leq d$. Since the $A^{i i}$ are symmetric, all of the $\lambda_{\mu}^{i}$ will be real. Furthermore, since the configurations of resolved particles that are used to generate the $A^{i i}$ are random, this means that the $\lambda_{\mu}^{i}$ are themselves random variables. Finally, due to spatial isotropy and the indistinguishability of the resolved particles, we can conclude that the $\lambda_{\mu}^{i}$ are distributed identically, regardless of $i$ or $\mu$. The eigenvalue distributions of the $A^{i i}$ obtained in this manner for the bold points in Figure 5.4 are shown in Figure 5.6. It is clear that the $A^{i i}$ are positive definite with overwhelming probabilty.

We now arrive at the final form for the approximate equations of motion of the resolved particles under the linear friction approximation:

$$
\begin{aligned}
\frac{d}{d t} \bar{q}_{i} & =\frac{1}{m} \bar{p}_{i} \\
\frac{d}{d t} \bar{p}_{i} & =-\nabla_{\bar{q}_{i}} \widehat{\phi}(\bar{q})-A^{i i}(\bar{q}) \bar{p}_{i}+G^{i i}(\bar{q}) W^{i}(t),
\end{aligned}
$$

where the $A^{i i}$ are as in (5.57), and the $G^{i i}$ satisfy the fluctuation-dissipation relation

$$
G^{i i}\left(G^{i i}\right)^{T}=2 m k_{B} T A^{i i}
$$




\subsection{Finding the Optimal Microscopic Timescale}

The value of the microscopic timescale $\tau$ cannot be determined directly from the steps leading to the short-memory approximation (5.4). However, we can obtain a rough estimate of its order of magnitude by other means. In this section, we use the requirement that the velocity autocorrelation functions of the full and reduced systems coincide as much as possible to derive estimates for $\tau$ in the low-density and high density limits. These results will be valid in $d=3$, though the method may be easily generalised to an arbitrary number of dimensions.

\subsubsection{Low Density}

When the density $\rho$ of the full system is low, the resolved and unresolved densities $\widehat{\rho}$ and $\widetilde{\rho}$ will be low as well. In that case, subsequent collisions between a tagged particle and the surrounding particles should be approximately uncorrelated in both the full system and the reduced system. We can therefore use a first-principles kinetic argument to derive an expression for the average net force on a given particle due to collisions with its neighbors.

Consider a tagged particle moving with momentum $p$ in the full system at equilibrium, where the density of surrounding particles is $\rho$, and the temperature is $T$. The change in the particle's momentum during a small time interval can be written as:

$$
\begin{aligned}
d p & =\left(\begin{array}{c}
\text { Number of particles } \\
\text { encountered }
\end{array}\right) \times\left(\begin{array}{c}
\text { Average change in } \\
\text { momentum per encounter }
\end{array}\right) \\
& =d N \frac{d p}{d N} .
\end{aligned}
$$

The number of particles encountered $d N$ can be estimated by considering the volume of influence of the particle when it moves a distance $d x$, as well as the number of particles contained in that volume (see Figure 5.7):

$$
\begin{aligned}
d N & \approx d x \pi(\alpha \sigma)^{2} \rho \\
& =\frac{|p|}{m} d t \pi \alpha^{2} \sigma^{2} \rho .
\end{aligned}
$$

Here $d t$ is a small increment of time, and $\pi(\alpha \sigma)^{2}$ is the total scattering cross section area of the interparticle potential. We have defined $\alpha \sigma$ to be the effective range of the potential, where $\sigma$ is defined in Chapter 3 as the scale length of the potential, and $\alpha$ is a dimensionless parameter of order unity. Note that the scattering cross section must be finite, since the potential is assumed to fall off faster than $1 / r^{d}$.

The change in momentum per encounter $d p / d N$ can be estimated by noting that, since all of the particles have equal mass, the tagged particle's momentum will change by an amount roughly equal to the momentum of the particle it collides with. This will have a magnitude of approximately $\sqrt{m k_{B} T}$ at thermal equilibrium. Furthermore, we expect the average change in momentum to be directed opposite the momentum of the tagged particle. Thus:

$$
\frac{d p}{d N} \approx-\sqrt{m k_{B} T} \frac{p}{|p|}
$$

Combining equations (5.64), (5.65), and (5.66) yields

$$
d p \approx-\pi \alpha^{2} \sigma^{2} \rho \sqrt{\frac{k_{B} T}{m}} p d t,
$$




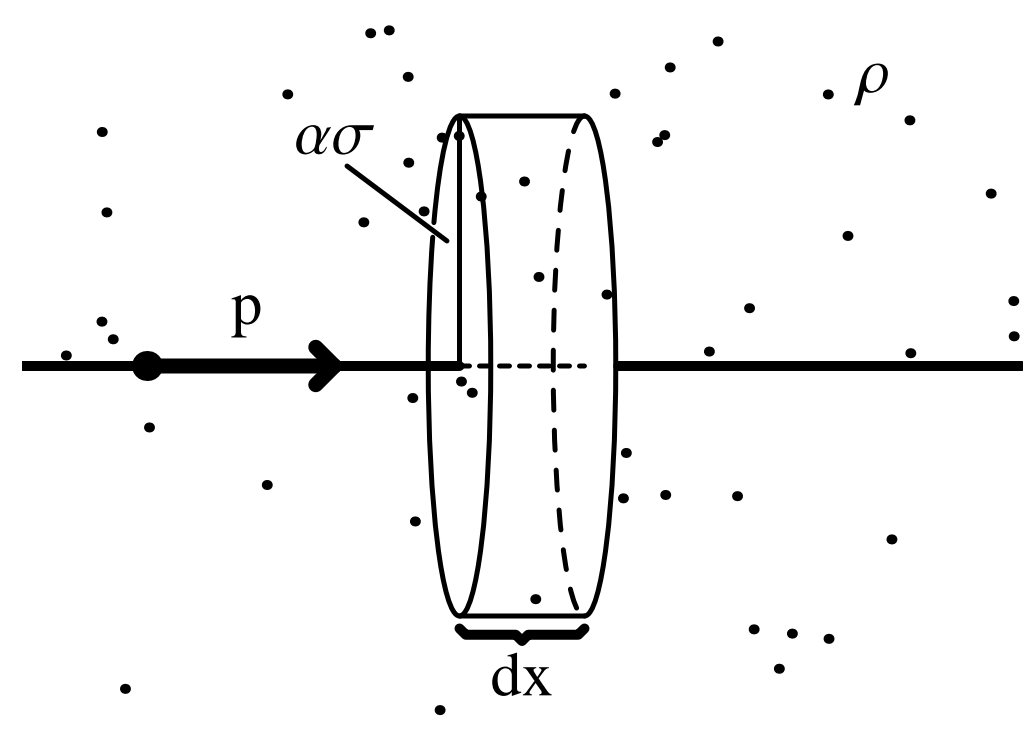

Figure 5.7: Schematic diagram depicting the volume of influence of a particle moving at momentum $p$ that travels a distance $d x$.

which implies that the equation of motion of a single particle in the full system at low density can be approximated by

$$
\frac{d}{d t} p(t)=-\pi \alpha^{2} \sigma^{2} \rho \sqrt{\frac{k_{B} T}{m}} p(t)+\text { noise } .
$$

Here "noise" represents mean-zero deviations from the average value of $d p / d N$ from (5.66).

The velocity autocorrelation function $C_{v v}(t)$ of the full system is given in terms of the tagged particle's momentum by

$$
C_{v v}(t)=\frac{1}{3 m^{2}}\langle y \cdot p(t)\rangle_{C} \cdot
$$

Here $p(0)=y$ is the initial value of the momentum, and $\langle\ldots\rangle_{C}$ represents an average over the canonical distribution. If we multiply both sides of $(5.67)$ by $\left(1 / 3 m^{2}\right) y$, and take a canonical average, we arrive at

$$
\frac{d}{d t} C_{v v}(t)=-\pi \alpha^{2} \sigma^{2} \rho \sqrt{\frac{k_{B} T}{m}} C_{v v}(t)
$$

where we have made the assumption that the noise term is uncorrelated with $y$. Combining (5.68) with that fact that $C_{v v}(0)=k_{B} T / m$ at equilibrium allows us to write $C_{v v}(t)$ as

$$
C_{v v}(t)=\frac{k_{B} T}{m} \exp \left(-\pi \alpha^{2} \sigma^{2} \rho \sqrt{\frac{k_{B} T}{m}} t\right) .
$$

Note that this expression cannot be accurate for large $t$, since it is known that in this limit the velocity autocorrelation function decays as $t^{-3 / 2}$ due to hydrodynamic effects [2]. However, for small $t$, it should suffice. 
Turning now to the reduced system with linear friction given by equation (5.62), we may write the equation of motion for the momentum of a tagged particle, for example particle 1 , as

$$
\frac{d}{d t} \bar{p}_{1}=-\nabla_{\bar{q}_{1}} \widehat{\phi}(\bar{q})-A^{11}(\bar{q}) \bar{p}_{1}+\text { noise } .
$$

The first term on the righthand side of (5.70) represents the net interaction of particle 1 with its neighbors via the renormalized pair potential. Since the density of the surrounding resolved particles $\widehat{\rho}$ is low, we may approximate this quantity by the same kinetic argument that lead us to (5.67), which yields:

$$
-\nabla_{\bar{q}_{1}} \widehat{\phi}(\bar{q}) \approx-\pi \widehat{\alpha}^{2} \sigma^{2} \widehat{\rho} \sqrt{\frac{k_{B} T}{m}} \bar{p}_{1} .
$$

Here $\widehat{\alpha} \sigma$ is the effective range of the renormalized pair potential, and $\widehat{\alpha}$ is a dimensionless quantity of order unity. Since the range of the renormalized potential tends to increase with increasing $\widetilde{\rho}$, we expect $\widehat{\alpha}$ to be a function of $\widetilde{\rho}$, and to be greater than or equal to $\alpha$. The second term in (5.70) may be simplified by noting that Figure 5.6a indicates that the matrices $A^{i i}$ are dominated by their leading diagonal contributions at low densities. This allows us to make the approximation that

$$
A^{11}(\bar{q}) \approx \frac{\tau}{m} \widetilde{\rho} \Gamma(\widetilde{\rho}) I
$$

in which case (5.70) becomes

$$
\frac{d}{d t} \bar{p}_{1} \approx-\left(\pi \widehat{\alpha}^{2} \sigma^{2} \widehat{\rho} \sqrt{\frac{k_{B} T}{m}}+\frac{\tau}{m} \widetilde{\rho} \Gamma(\widetilde{\rho})\right) \bar{p}_{1}+\text { noise } .
$$

Equation (5.72) implies that the velocity autocorrelation function $\widehat{C}_{v v}(t)$ of the reduced system may be written as

$$
\widehat{C}_{v v}(t)=\frac{k_{B} T}{m} \exp \left[-\left(\pi \widehat{\alpha}^{2} \sigma^{2} \widehat{\rho} \sqrt{\frac{k_{B} T}{m}}+\frac{\tau}{m} \widetilde{\rho} \Gamma(\widetilde{\rho})\right) t\right] .
$$

Since the correlation functions of the reduced system are meant to mimic those of the full system as well as possible, we equate the two exponents in (5.69) and (5.73), which allows us to solve for $\tau_{\ell d}$, the low density estimate for $\tau$ :

$$
\tau_{\ell d}=\frac{\pi \sigma^{2} \sqrt{m k_{B} T}\left(\alpha^{2} \rho-\widehat{\alpha}^{2} \widehat{\rho}\right)}{\widetilde{\rho} \Gamma(\widetilde{\rho})} .
$$

Since $\rho, \widehat{\rho}$, and $\widetilde{\rho}$ are not independent of one another, we may consider $\tau_{\ell d}$ to be a function of any two of them. For later convenience, we choose to regard this and all other expressions for $\tau$ as functions of $\rho$ and $\widetilde{\rho}$.

It may be argued that we have simply replaced one unknown parameter, $\tau$, by the two unknown parameters $\alpha$ and $\widehat{\alpha}$. However, there are several advantages to this formulation. Since both $\alpha$ and $\widehat{\alpha}$ are of order unity, we know their order of magnitude. The same cannot be said for $\tau$. Furthermore, because $\widehat{U} \rightarrow U$ as $\widetilde{\rho} \rightarrow 0$, we expect $\widehat{\alpha} \rightarrow \alpha$ in this limit as well. Thus, in the low density limit, we should be able to approximate the $\widetilde{\rho}$ dependence of $\widehat{\alpha}$ by the first terms of a series around $\widetilde{\rho}=0: \widehat{\alpha} \approx \alpha+\gamma \sigma^{3} \widetilde{\rho}$. Here a factor of $\sigma^{3}$ has been introduced to keep $\widehat{\alpha}$ dimensionless, and $\gamma$ is a small, dimensionless parameter. The original problem of finding a different value of $\tau$ for every value of $\rho$ and $\widetilde{\rho}$ has therefore been reduced to the determination of the two dimensionless quantities $\alpha$ and $\gamma$. 


\subsubsection{High Density}

At high densities, matters become more complicated, as subsequent interparticle collisions become highly correlated in a way that is difficult to model analytically. However, we can make some progress by noting that at high densities dynamical autocorrelation functions are better approximated by their Taylor expansions around $t=0$ than at low densities. In other words, the first few terms of the Taylor series track the behavior of the true function for much larger values of $t$ at high densities. By examining the leading terms of the expansions of the velocity autocorrelation functions in the full and reduced systems, we can extract an estimate for the high density behavior of $\tau$.

The velocity autocorrelation function $C_{v v}(t)$ of the full system can be written as a Taylor series as follows:

$$
\begin{aligned}
C_{v v}(t) & =\frac{1}{3 m^{2}}\left\langle y_{1} \cdot p_{1}(t)\right\rangle_{C} \\
& =\frac{1}{3 m^{2}}\left\langle y_{1} \cdot e^{t L} y_{1}\right\rangle_{C} \\
& =\frac{1}{3 m^{2}}\left\{\left\langle\left|y_{1}\right|^{2}\right\rangle_{C}+\frac{t^{2}}{2 !}\left\langle y_{1} \cdot L^{2} y_{1}\right\rangle_{C}+O\left(t^{4}\right)\right\} .
\end{aligned}
$$

Since all particles are equivalent, we have chosen to write $C_{v v}(t)$ in terms of the momentum of particle 1 for convenience. Note that only terms even in $t$ appear in (5.75) due to timereversal symmetry. We can simplify the terms in (5.75) as follows:

$$
\left\langle\left|y_{1}\right|^{2}\right\rangle_{C}=3 m k_{B} T
$$

and

$$
\begin{aligned}
\left\langle y_{1} \cdot L^{2} y_{1}\right\rangle_{C} & =-\left\langle y_{1} \cdot L \nabla_{x_{1}} \phi(x)\right\rangle_{C} \\
& =-\frac{1}{m} \sum_{i=1}^{N}\left\langle\left(y_{1} \cdot \nabla_{x_{1}}\right)\left(y_{i} \cdot \nabla_{x_{i}}\right) \phi(x)\right\rangle_{C} \\
& =-k_{B} T\left\langle\triangle_{x_{1}} \phi(x)\right\rangle_{C} \\
& =-k_{B} T(N-1) \frac{\int d x_{1} \int d x_{2} \triangle_{x_{1}} U_{12} \int d x_{3} \ldots d x_{N} e^{-\beta \phi}}{\int d x e^{-\beta \phi}} \\
& =-k_{B} T \rho \int d x_{1} \triangle_{x_{1}} U\left(\left|x_{1}\right|\right) g^{(2)}\left(\left|x_{1}\right|, \rho, T\right) \\
& =-3 k_{B} T \rho \Gamma(\rho),
\end{aligned}
$$

where in the third equality we have used the fact that $\left\langle y_{i \mu} y_{j \nu}\right\rangle_{C}=\delta_{i j} \delta_{\mu \nu} m k_{B} T$, and in the fifth equality we have used the definition of $g^{(2)}$. The function $\Gamma(\rho)$ is as defined in (5.50). These expressions allow us to write (5.75) as

$$
C_{v v}(t)=\frac{k_{B} T}{m}\left\{1-\frac{\rho \Gamma(\rho)}{m} \frac{t^{2}}{2 !}+O\left(t^{4}\right)\right\} .
$$

In order to find the leading terms in the series expansion of $\widehat{C}_{v v}(t)$, we start with (5.70). Replacing $A^{11}$ with its dominant diagonal contribution as in (5.71), multiplying both sides 
on the left by $\left(1 / 3 m^{2}\right) \widehat{y}_{1}$, and taking an average over the renormalized canonical distribution gives us

$$
\frac{d}{d t} \widehat{C}_{v v}(t)=-\frac{\tau}{m} \widetilde{\rho} \Gamma(\widetilde{\rho}) \widehat{C}_{v v}(t)-\frac{1}{3 m^{2}}\left\langle\widehat{y} \cdot \nabla_{\bar{q}_{1}} \widehat{\phi}(\bar{q})\right\rangle_{\widehat{C}}
$$

which may be solved for $\widehat{C}_{v v}(t)$ to yield

$$
\begin{aligned}
\widehat{C}_{v v}(t)=\frac{k_{B} T}{m} \exp (- & \left.\frac{\tau}{m} \widetilde{\rho} \Gamma(\widetilde{\rho}) t\right) \\
& \quad-\frac{1}{3 m^{2}} \int_{0}^{t} d s \exp \left(-\frac{\tau}{m} \widetilde{\rho} \Gamma(\widetilde{\rho})(t-s)\right)\left\langle\widehat{y}_{1} \cdot \nabla_{\bar{q}_{1}} \widehat{\phi}(\bar{q}(s))\right\rangle_{\widehat{C}}
\end{aligned}
$$

The integral term in (5.77) contributes only to second order in $t$, as can be seen by expanding the integrand:

$$
\begin{aligned}
\int_{0}^{t} d s \exp \left(-\frac{\tau}{m} \widetilde{\rho} \Gamma(\widetilde{\rho})(t-s)\right) & \left\langle\widehat{y}_{1} \cdot \nabla_{\bar{q}_{1}} \widehat{\phi}(\bar{q}(s))\right\rangle_{\widehat{C}} \\
& =\int_{0}^{t} d s\{1+O(t, s)\} \times\{\underbrace{\left\langle\widehat{y}_{1} \cdot \nabla_{\widehat{x}_{1}} \widehat{\phi}(\widehat{x})\right\rangle_{\widehat{C}}}_{=0}+O(s)\} \\
& =O\left(t^{2}\right) .
\end{aligned}
$$

This allows us to write (5.77) as

$$
\widehat{C}_{v v}(t)=\frac{k_{B} T}{m}\left\{1-\frac{\tau}{m} \widetilde{\rho} \Gamma(\widetilde{\rho}) t+O\left(t^{2}\right)\right\} .
$$

Comparing equations (5.76) and (5.78) indicates that it is impossible for $C_{v v}(t)$ and $\widehat{C}_{v v}(t)$ to coincide exactly at high densities, since the former is even in $t$, whereas the series of the latter contains nonzero contributions to all orders in $t$. However, we can ensure that the curves $C_{v v}(t)$ and $\widehat{C}_{v v}(t)$ are close to one another in the neighborhood of $t=0$ by requiring the values of the leading-order coefficients in their series expansions to satisfy

$$
\frac{\tau}{m} \widetilde{\rho} \Gamma(\widetilde{\rho}) \sim\left(\frac{\rho \Gamma(\rho)}{m}\right)^{\frac{1}{2}} .
$$

Treating (5.79) as a literal equality allows us to solve for $\tau_{h d}$, the high density estimate for $\tau$ :

$$
\tau_{h d}=\frac{(m \rho \Gamma(\rho))^{\frac{1}{2}}}{\widetilde{\rho} \Gamma(\widetilde{\rho})} .
$$

\subsubsection{Comparison to Optimal Values}

The extensive approximations we have made in order to arrive at the low and high density expressions (5.74) and (5.80) imply that they cannot be used for anything more than orderof-magnitude estimates of $\tau$. In order to test this, we can directly determine the optimal values of $\tau$ for various values of $\rho$ and $\widetilde{\rho}$, by the following procedure: Let $C_{v v}(t, \rho, T)$ and $\widehat{C}_{v v}(t, \tau, \widetilde{\rho}, \rho, T)$ be the velocity autocorrelation functions of the full system and the reduced system, respectively. We include here the parametric dependence of both of these functions 
upon such quantities as $\rho, T, \tau$, etc. The "distance" between these two correlation functions is defined as

$$
I(\tau, \rho, \widetilde{\rho}, T) \equiv \int_{0}^{t_{\max }} d t\left[C_{v v}(t, \rho, T)-\widehat{C}_{v v}(t, \tau, \widetilde{\rho}, \rho, T)\right]^{2}
$$

Here " $t_{\max }$ " is a somewhat arbitrary timescale equal to the time below which all of the "interesting" behavior of the full system's autocorrelation function occurs. For example, the $\rho^{*}=1.1$ autocorrelation function of the full system depicted in Figure 4.22 would have $t_{\max } \approx 0.7$.

Our estimate of the optimal value of $\tau$ for a given $\rho, \widetilde{\rho}$, and $T$ is the value that minimizes $I(\tau, \rho, \tilde{\rho}, T)$. We can bracket this minimum within an acceptable tolerance by numerically simulating the linear friction equations of motion (5.62) for many values of $\tau$, via an algorithm to be described Section 5.7. The results of such a calculation for the Lennard-Jones system at reduced temperature $T^{*}=1.6$, and at various values of $\rho^{*}$ and $\widetilde{\rho}^{*}$, is shown in Figure 5.8, along with the low and high density estimates $\tau_{\ell d}$ and $\tau_{h d}$, as well as their average $\left(\tau_{\ell d}+\tau_{h d}\right) / 2$. We see that $\tau_{\ell d}$ and $\tau_{h d}$ give surprisingly good results at low and high densities, respectively. At moderate densities, however, neither estimate captures the correct value of $\tau$, which generally lies between them.

We should point out two things about this calculation. First, we find that the parameter values $\alpha \approx 1.36$ and $\gamma \approx 0.57$ yield the best fit of (5.74) to the low density results. Second, we find that $I(\tau, \rho, \widetilde{\rho}, T)$ is relatively insensitive to the value of $\tau$ near the minimum. Thus, there is a wide range of acceptable $\tau$ 's at any given density and temperature. This is illustrated by the vertical bars on each of the points in Figure 5.8.

\subsection{Optimal Prediction and Dissipative Particle Dynamics}

It is worth pausing at this point to note the similarities and differences between the linear friction approximation of this chapter as derived from OP, and another coarse-graining procedure known as dissipative particle dynamics (DPD) $[25,16,40]$. DPD is a technique for simulating a fluid on the mesoscopic scale that seeks to overcome the limitations of pure $\mathrm{MD}$, which is confined to microscopic time and length scales. A typical implementation of DPD consists of $M$ particles, with positions $q_{i}$ and $p_{i}$, that evolve according to a set of stochastic differential equations which are usually written as follows:

$$
\begin{aligned}
\frac{d}{d t} q_{i} & =\frac{1}{m} p_{i} \\
\frac{d}{d t} p_{i} & =\sum_{j \neq i}^{M}\left\{-\nabla_{q_{i}} \mathcal{U}\left(\left|q_{i j}\right|\right)-\frac{\gamma}{m} w_{D}\left(\left|q_{i j}\right|\right)\left[\hat{e}_{i j} \cdot\left(p_{i}-p_{j}\right)\right] \hat{e}_{i j}+\sigma w_{R}\left(\left|q_{i j}\right|\right) \hat{e}_{i j} \eta_{i j}(t)\right\} .
\end{aligned}
$$

The three terms in brackets above can be interpreted as a conservative force, a dissipative frictional force, and a random force, respectively. Here $\mathcal{U}$ is a radially symmetric interparticle potential, as is normally found in MD. In the second term, $\gamma$ is a positive constant, $w_{D}$ is a scalar weighting function, and $\hat{e}_{i j}$ is a unit vector along the line joining particle $j$ and particle $i$. In the last term, $\sigma$ is another positive constant, $w_{R}$ is another weighting function, and the $\eta_{i j}(t)$ are a family of scalar-valued white noise processes that satisfy

$$
\left\langle\eta_{i j}(t)\right\rangle=0 \quad \text { and } \quad\left\langle\eta_{i j}(t) \eta_{k \ell}\left(t^{\prime}\right)\right\rangle=\delta\left(t-t^{\prime}\right)\left(\delta_{i k} \delta_{j \ell}+\delta_{i \ell} \delta_{j k}\right) .
$$




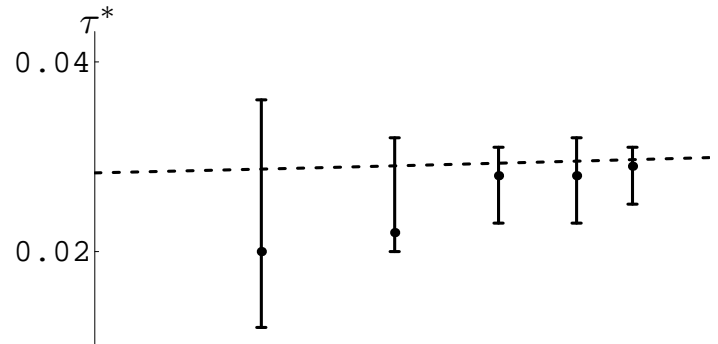

(a) $\rho^{*}=0.10$
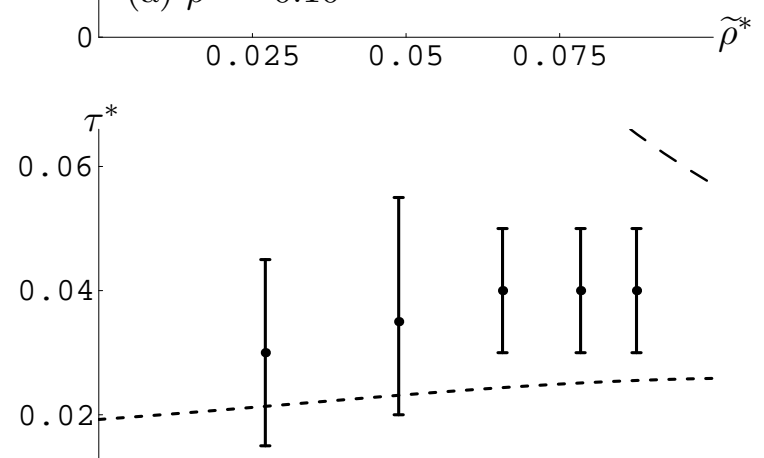

(c) $\rho^{*}=0.45$
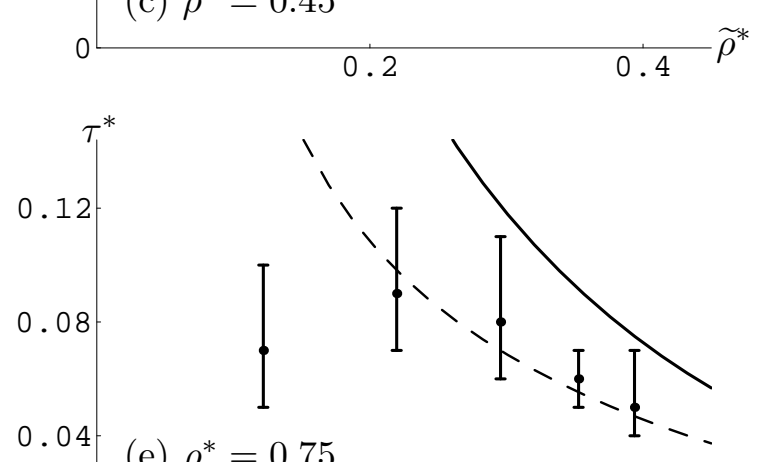

(e) $\rho^{*}=0.75$
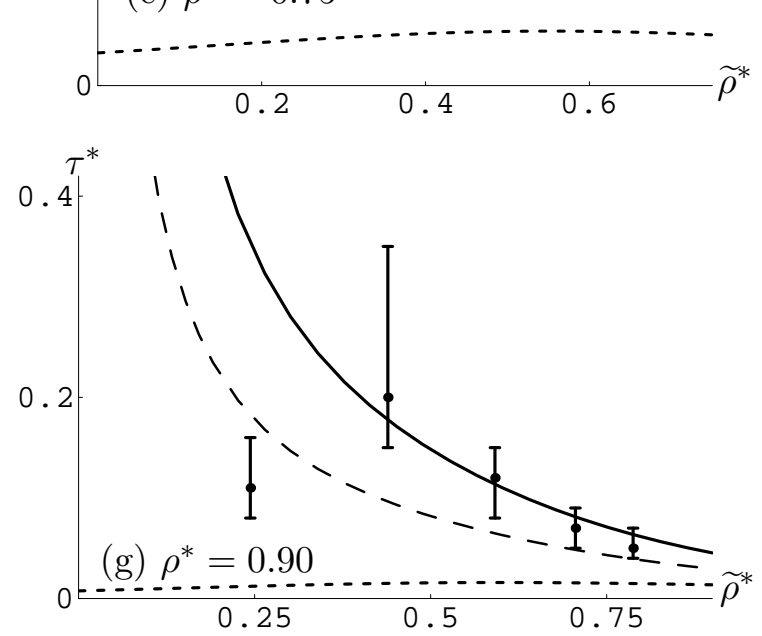

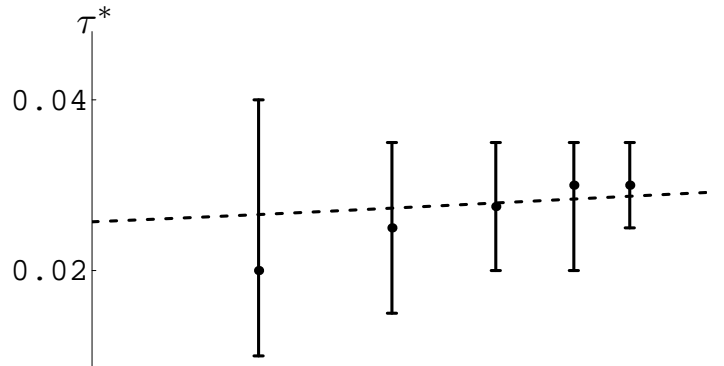

(b) $\rho^{*}=0.20$
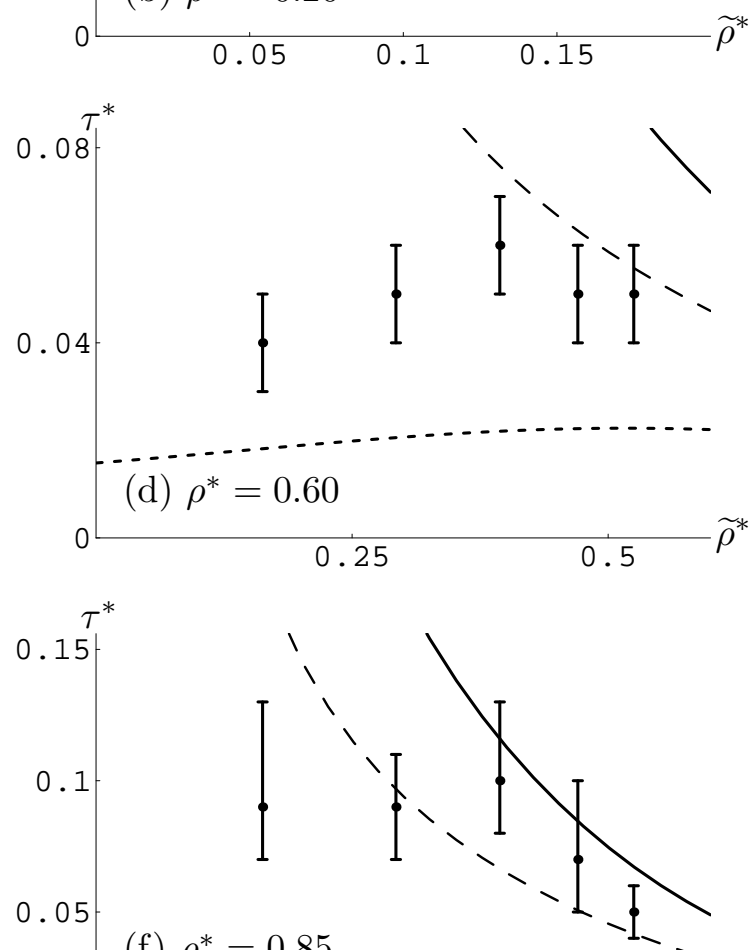

(f) $\rho^{*}=0.85$
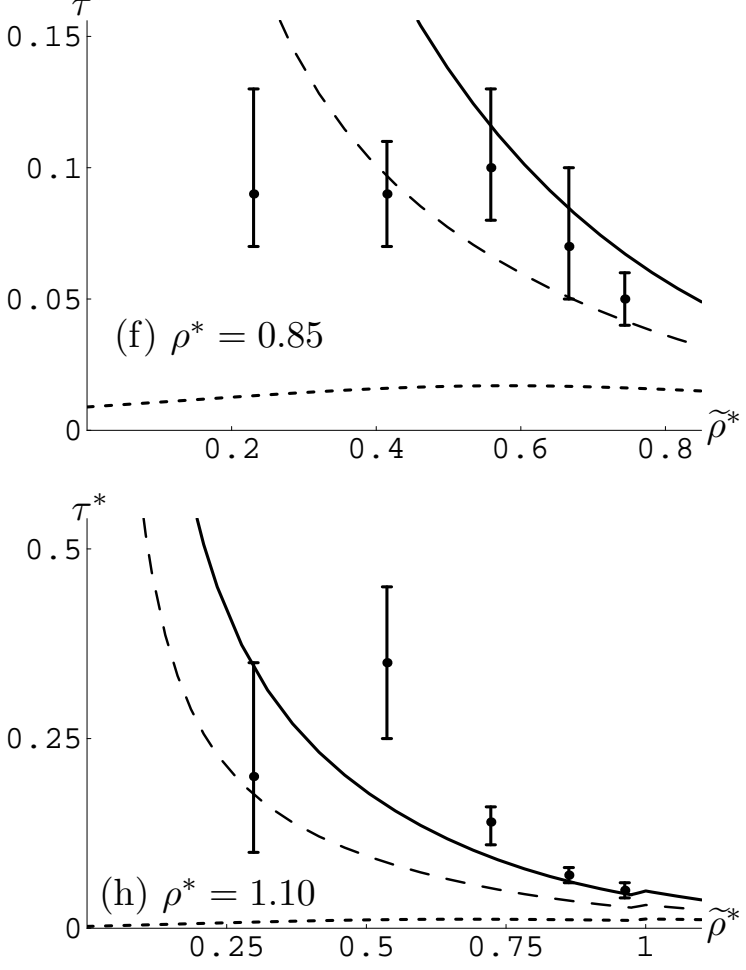

Figure 5.8: The microscopic timescale $\tau^{*}$ as a function of $\widetilde{\rho}^{*}$, for various values of $\rho^{*}$, at $T^{*}=1.6$. Points: numerically-determined optimal values (bars represent the acceptable interval for $\left.\tau^{*}\right)$. Dotted lines: low density estimate $\tau_{\ell d}$. Solid lines: high density estimate $\tau_{h d}$. Dashed lines: average of the low and high density estimates, $\frac{1}{2}\left(\tau_{\ell d}+\tau_{h d}\right)$. 
Note that all of the forces in (5.81) are pairwise forces directed along the line joining each pair of particles. Furthermore, (5.81) conserves both linear and angular momentum, though not energy. By using detailed balance, as well the requirement that the DPD system equilibrate to the canonical distribution associated with the Hamiltonian

$$
\mathcal{H} \equiv \sum_{i=1}^{M} \frac{\left|p_{i}\right|^{2}}{2 m}+\sum_{i<j}^{M} \mathcal{U}\left(\left|q_{i j}\right|\right),
$$

it can be shown that

$$
w_{D}(r)=w_{R}^{2}(r) \equiv w(r) \quad \text { and } \quad k_{B} T=\frac{\sigma^{2}}{2 \gamma} .
$$

This implies that $w(r)$ must be a positive function. Numerical results tend to be fairly insensitive to the exact form of $w(r)$, and so it is usually chosen for computational convenience. A typical choice is

$$
w(r)=\left\{\begin{array}{ll}
2\left(1-\frac{r}{r_{c}}\right) & r<r_{c} \\
0 & r \geq r_{c}
\end{array} .\right.
$$

We can rearrange (5.81) somewhat to put it into a more suggestive form by noting that $\left[\hat{e}_{i j} \cdot\left(p_{i}-p_{j}\right)\right] \hat{e}_{i j}=P^{i j}\left(p_{i}-p_{j}\right)$, where $P^{i j}$ is as defined in (5.40). Furthermore, it is easy to show that the quantity $\hat{e}_{i j} \eta_{i j}(t)$, subject to the constraints (5.82), is identical, in the statistical sense, to $P^{i j} \tilde{W}^{i j}(t)$, where the $\tilde{W}^{i j}$ are a family of $d$-dimensional white noise processes that satisfy

$$
\left\langle\tilde{W}^{i j}(t)\right\rangle=0 \quad \text { and } \quad\left\langle\tilde{W}_{\mu}^{i j}(t) \tilde{W}_{\nu}^{k \ell}\left(t^{\prime}\right)\right\rangle=\delta\left(t-t^{\prime}\right) \delta_{\mu \nu}\left(\delta_{i k} \delta_{j \ell}-\delta_{i \ell} \delta_{j k}\right) .
$$

This, along with the constraint (5.83), allows us to rewrite (5.81) as

$$
\begin{aligned}
\frac{d}{d t} q_{i} & =\frac{1}{m} p_{i} \\
\frac{d}{d t} p_{i} & =\sum_{j \neq i}^{M}\left\{-\nabla_{q_{i}} \mathcal{U}\left(\left|q_{i j}\right|\right)-\frac{\gamma}{m} w\left(\left|q_{i j}\right|\right) P^{i j}\left(p_{i}-p_{j}\right)+\left[2 k_{B} T \gamma w\left(\left|q_{i j}\right|\right)\right]^{1 / 2} P^{i j} \tilde{W}^{i j}(t)\right\} .
\end{aligned}
$$

We now turn to the linear friction approximation, in the form

$$
\begin{aligned}
& \frac{d}{d t} \bar{q}_{i}=\frac{1}{m} \bar{p}_{i} \\
& \frac{d}{d t} \bar{p}_{i}=-\sum_{j \neq i}^{M} \nabla_{\bar{q}_{i}} \widehat{U}\left(\left|\bar{q}_{i j}\right|\right)+\sum_{j=1}^{M}\left\{-A^{i j}(\bar{q}) \bar{p}_{j}+G^{i j}(\bar{q}) \dot{W}^{i j}(t)\right\},
\end{aligned}
$$

where the $A^{i j}$ and $G^{i j}$ are constrained by (5.61). If we use the expressions (5.41) and (5.57) for the $A^{i j}$, then we can rearrange (5.85) to yield:

$$
\begin{aligned}
& \frac{d}{d t} \bar{q}_{i}= \frac{1}{m} \bar{p}_{i} \\
& \frac{d}{d t} \bar{p}_{i}=\sum_{j \neq i}^{M}\left\{-\nabla_{\bar{q}_{i}} \widehat{U}\left(\left|\bar{q}_{i j}\right|\right)-\frac{\tau}{m} \zeta\left(\bar{q}_{i j}\right)\left(\bar{p}_{i}-\bar{p}_{j}\right)+G^{i j}(\bar{q}) \dot{W}^{i j}(t)\right\} \\
&-\frac{\tau}{m} \Omega^{i}(\bar{q}) \bar{p}_{i}+G^{i i}(\bar{q}) \dot{W}^{i i}(t),
\end{aligned}
$$


where

$$
\zeta\left(\bar{q}_{i j}\right) \equiv-\frac{\delta \widehat{U}_{i j}^{\prime}}{\left|\bar{q}_{i j}\right|} I+\left(\frac{\delta \widehat{U}_{i j}^{\prime}}{\left|\bar{q}_{i j}\right|}-\delta \widehat{U}_{i j}^{\prime \prime}\right) P^{i j}
$$

and

$$
\Omega^{i}(\bar{q}) \equiv \widetilde{\rho} \Gamma(\widetilde{\rho}, T) I+\sum_{j \neq i}^{M} \widetilde{\rho}\left[\xi_{i j}^{\perp} I+\left(\xi_{i j}^{\|}-\xi_{i j}^{\perp}\right) P^{i j}\right] .
$$

There are many striking similarities and differences between (5.84) and (5.86). They both share a pairwise conservative force term, a momentum-conserving friction term, and a pairwise white noise term. However, the noise term in (5.86) is not momentum conserving, because the $\dot{W}^{i j}$ are entirely uncorrelated with one another. Furthermore, (5.86) contains an additional one-particle friction term and an additional noise term, neither of which conserves momentum.

The fact that the linear friction approximation does not conserve momentum implies that it will not be able to reproduce macroscopic hydrodynamics, since local momentum conservation is necessary for a momentum transport equation to exist. While the DPD method does conserve local momentum, this is partially a result of the fact that it was not originally derived from any underlying microscopic model (though attempts have been made to remedy this [18]), but was instead an ad-hoc attempt at coarse-graining that was designed to conserve momentum by construction. This also results from differences in the "philosophies" of OP and DPD. When OP is applied to MD, the resolved particles are actual particles of the original system, whereas in DPD, the "particles" are really meant to represent mesoscopic "lumps" of fluid containing many of the underlying microscopic molecules.

This conceptual difference can be illustrated by an analogy with simple two-dimensional spin systems. In such systems, in which there is a lattice of interacting spins, one often wants to make a type of coarse-grained approximation by blocking together several neighboring spins and performing a renormalization step in which each block is replaced by a single spin. This has the effect of altering the system's Hamiltonian. A spin system that possesses only nearest-neighbor pair interactions will, in general, gain longer-ranged interactions that include multi-particle effects after renormalization. This is illustrated schematically in Figure 5.9. This effect is analogous to what happened in Chapter 4 during renormalization of the interparticle potential.

When performing the renormalization step depicted in Figure 5.9, there are various methods used to determine the value of the spin that remains from each block. For example, one might use a "majority rules" or "grouping" technique, by which the value of the new spin is positive if the majority of the spins in the original block were positive, and vice-versa. It should be clear that in this case the new spin is really a composite of the original spins. Alternatively, one might choose a "representative" technique, in which we take the value of the new spin to be equal to the value of one particular spin from the original block (for example, the center spin). In this instance, the new spin is the same as one of the old spins. DPD is a molecular analog of the grouping method of coarse-graining, whereas OP, when applied to MD, is analogous to the representative method. 

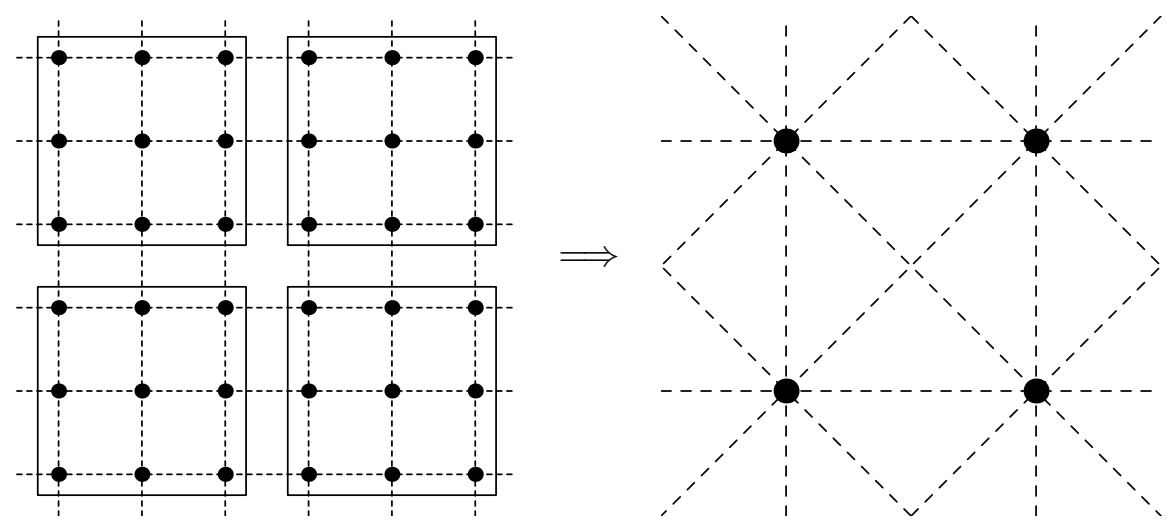

Figure 5.9: Block renormalization of a two-dimensional spin system. Dashed lines represent spin-spin interactions.

\subsection{Numerical Tests of the Linear Friction Approximation}

\subsubsection{Numerical Algorithm}

Before we can test the linear friction approximation to see if it adequately reproduces various thermodynamic quantities, we must construct a suitable numerical scheme for the equations of motion (5.62), which we write here as

$$
\begin{aligned}
\frac{d}{d t} \bar{q}_{i} & =\frac{1}{m} \bar{p}_{i} \\
\frac{d}{d t} \bar{p}_{i} & =F_{i}^{c}-A^{i i} \bar{p}_{i}+G^{i i} \dot{W}^{i}(t),
\end{aligned}
$$

where

$$
F_{i}^{c} \equiv-\sum_{j \neq i}^{M} \nabla_{\bar{q}_{i}} \widehat{U}\left(\left|\bar{q}_{i j}\right|\right)
$$

is the conservative force on particle $i$.

As we will see, it is possible to construct an algorithm that is second-order in the timestep $\Delta t$, requires only one force evaluation per timestep, and is reasonably simple to execute. We start with the analog of the velocity Verlet scheme (3.15) for (5.87):

$$
\begin{aligned}
& \bar{p}_{i}\left(t+\frac{\Delta t}{2}\right)=\bar{p}_{i}(t)+\frac{\Delta t}{2}\left[F_{i}^{c}(t)-A^{i i}(t) \bar{p}_{i}(t)\right]+\left(\frac{\Delta t}{2}\right)^{1 / 2} G^{i i}(t) Z_{i}(t) \\
& \bar{q}_{i}(t+\Delta t)=\bar{q}_{i}(t)+\frac{\Delta t}{m} \bar{p}_{i}\left(t+\frac{\Delta t}{2}\right) \\
& F_{i}^{c}(t+\Delta t)=F_{i}^{c}\left(\bar{q}_{i}(t+\Delta t)\right) \\
& A^{i i}(t+\Delta t)=A^{i i}(\bar{q}(t+\Delta t)) \\
& G^{i i}(t+\Delta t) \text { by Cholesky decomposition from } G^{i i}\left(G^{i i}\right)^{T}=2 m k_{B} T A^{i i} \\
& \bar{p}_{i}(t+\Delta t)=\bar{p}_{i}\left(t+\frac{\Delta t}{2}\right)+\frac{\Delta t}{2}\left[F_{i}^{c}(t+\Delta t)-A^{i i}(t+\Delta t) \bar{p}_{i}(t+\Delta t)\right] \\
&+\left(\frac{\Delta t}{2}\right)^{1 / 2} G^{i i}(t+\Delta t) Z_{i}\left(t+\frac{\Delta t}{2}\right) .
\end{aligned}
$$


Here the $Z_{i}(t)$ and $Z_{i}\left(t+\frac{\Delta t}{2}\right)$ are $d$-dimensional vectors whose components are independent, identically-distributed random Gaussian numbers with mean zero and unit variance. These, along with the factors $\left(\frac{\Delta t}{2}\right)^{1 / 2}$, follow from the fact that

$$
\int_{t}^{t+\frac{\Delta t}{2}} d s \dot{W}_{\mu}^{i}(s)=\left(\frac{\Delta t}{2}\right)^{1 / 2} Z
$$

where $Z$ is Gaussian, mean zero, and has unit variance.

There are a number of difficulties that must be overcome in order to make the algorithm (5.88) practical. First, the implicit step (4) must be rendered explicit. Solving for $\bar{p}_{i}(t+\Delta t)$ yields

$$
\begin{aligned}
\bar{p}_{i}(t+\Delta t)=\left(I+\frac{\Delta t}{2} A^{i i}(t+\Delta t)\right)^{-1}\left[\bar{p}_{i}\left(t+\frac{\Delta t}{2}\right)+\frac{\Delta t}{2} F_{i}^{c}(t+\Delta t)\right. & \\
& \left.+\left(\frac{\Delta t}{2}\right)^{1 / 2} G^{i i}(t+\Delta t) Z_{i}\left(t+\frac{\Delta t}{2}\right)\right] .
\end{aligned}
$$

Since we are only trying to construct a second-order scheme, we can avoid performing $M$ matrix inversions per timestep, by Taylor exanding as follows:

$$
\left(I+\frac{\Delta t}{2} A^{i i}(t+\Delta t)\right)^{-1}=I-\frac{\Delta t}{2} A^{i i}(t+\Delta t)+\left(\frac{\Delta t}{2} A^{i i}(t+\Delta t)\right)^{2}+O\left(\Delta t^{3}\right) .
$$

Also, we must guard against the event that $A^{i i}$ is not positive definite. Figure 5.6(b) implies that we may expect rare configurations of the resolved particles to make this happen a few times during a simulation, especially at moderate values of $\widehat{\rho}$ and $\widetilde{\rho}$. If $A^{i i}$ is not positive definite, it raises the possibility of negative friction, which makes the system (5.87) highly unstable. We can remedy this by introducing a step into our algorithm in which we check to see if $A^{i i}$ is positive definite. This test can be performed concurrent with the Cholesky decomposition step in which we calculate $G^{i i}$. If it is not, we simply set both the matrices $A^{i i}$ and $G^{i i}$ equal to zero. 
Taking the above details into account, we can write our modified Verlet scheme as:

$$
\begin{aligned}
\bar{p}_{i}\left(t+\frac{\Delta t}{2}\right) & =\bar{p}_{i}(t)+\frac{\Delta t}{2}\left[F_{i}^{c}(t)-A^{i i}(t) \bar{p}_{i}(t)\right]+\left(\frac{\Delta t}{2}\right)^{1 / 2} G^{i i}(t) Z_{i}(t) \\
\bar{q}_{i}(t+\Delta t) & =\bar{q}_{i}(t)+\frac{\Delta t}{m} \bar{p}_{i}\left(t+\frac{\Delta t}{2}\right) \\
F_{i}^{c}(t+\Delta t) & =F_{i}^{c}\left(\bar{q}_{i}(t+\Delta t)\right) \\
A^{i i}(t+\Delta t) & =A^{i i}(\bar{q}(t+\Delta t))
\end{aligned}
$$

Check $A^{i i}(t+\Delta t)$. If it is positive definite, then calculate $G^{i i}(t+\Delta t)$ via Cholesky decomposition from $G^{i i}\left(G^{i i}\right)^{T}=2 m k_{B} T A^{i i}$.

Otherwise, set $A^{i i}=G^{i i}=0$.

$$
\begin{aligned}
p^{*} & =\bar{p}_{i}\left(t+\frac{\Delta t}{2}\right)+\frac{\Delta t}{2} F_{i}^{c}(t+\Delta t) \\
& +\left(\frac{\Delta t}{2}\right)^{1 / 2} G^{i i}(t+\Delta t) Z_{i}\left(t+\frac{\Delta t}{2}\right) \\
p^{* *} & =\frac{\Delta t}{2} A^{i i}(t+\Delta t) p^{*} \\
p^{*} & =p^{*}-p^{* *} \\
\bar{p}_{i}(t+d t) & =p^{*}+\frac{\Delta t}{2} A^{i i}(t+\Delta t) p^{* *} .
\end{aligned}
$$

Here $p^{*}$ and $p^{* *}$ are temporary dummy variables, and steps (4)-(7) simply describe the most efficient way of performing the matrix multiplications by various powers of $\frac{\Delta t}{2} A^{i i}$.

The method (5.89) is second-order, fast, and requires only one force evaluation per timestep (in step (3)). Furthermore, it preserves the temperature well, which is vital if we are to equilibrate to the canonical equilibrium.

\subsubsection{Results}

The linear friction approximation (LFA), as given in (5.87), was tested via the algorithm (5.89), and with the same parameter values used in Section 4.5.2 to investigate FOOP and the Galerkin model. The values chosen for the $\tau$ 's were determined by a least-square fit to the velocity autocorrelation data, as described in Section 5.5.3.

The relative runtimes of the LFA, as defined by (??), are shown in Figure ?? for each value of $M$ considered. A comparison with Figures ?? and ?? immediately reveals that the LFA requires significantly longer to simulate than either FOOP or the Galerkin system. In fact, at the highest densities, only the $M=500$ system runs faster than the full system. This is entirely due to the increased computational overhead inherent in the LFA scheme (5.89). In particular, at each timestep there are $M$ Cholesky decompositions (step (3)) and $2 M$ matrix multiplications (steps (5) and (7)) to perform, neither of which is present in the modified velocity Verlet algorithm (3.22) used in Chapter 4. Nevertheless, even with these handicaps, the LFA allows considerable reduction in the runtime for low-to-moderate densities and for low values of $M$. 


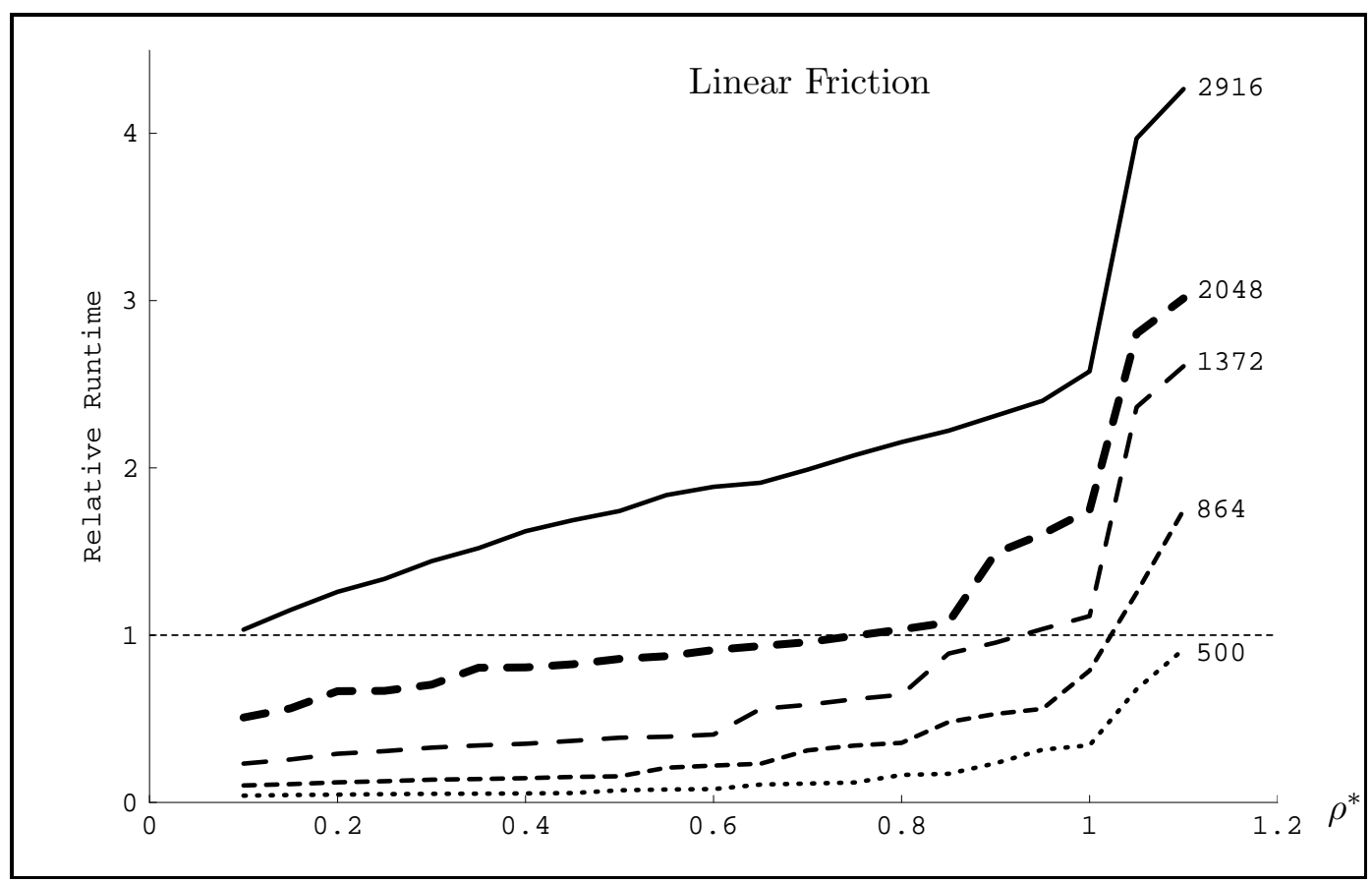

Figure 5.10: Relative runtimes of the linear friction systems as functions of reduced density, and for various values of $M$

\section{Static Averages}

As before, equations (4.51), (4.52), and (4.57) were used to evaluate the energy per particle, pressure, and heat capacity per particle, respectively, and the results are shown in Figures 5.11, 5.12, and 5.13. A comparison with Figures 4.12, 4.14, and 4.16 in Chapter 4 shows that the results are fairly similar to those obtained with FOOP. Although the heat capacity is still plagued by noise for the same reasons as explained in the previous chapter, the performance of the LFA system in determining the energy and pressure is superior to that of the Galerkin model. It is important to note, however, that there is a greater degree of irregularity in the data obtained from the LFA model, especially at high densities or low $M$ values. The reasons for this are subtle. MD simulations are often plagued by rare events, i.e. unlikely configurations of particles that cause two or more of them to approach one another extremely closely during a single timestep. This creates an unphysical blowup of local particle speeds, which, though rare, has an impact on the calculation of global quantities such as energy or pressure, since it tends to drive the system away from canonical equilibrium. Clearly, this source of error will be enhanced at high densities, due to the greater likelihood of manyparticle encounters. Furthermore, a random force added to the system, such as the one in the linear friction model, will have a similar effect, because the discretization of a white noise process adds kicks of potentially arbitrary size to the coordinates of the system at each timestep. Since the magnitude of the random force scales roughly linearly with $\widetilde{\rho}$ in the LFA, the size of this effect will tend to be greater when either $\rho$ is large or $M$ is small. Thus, the LFA has two sources of harmful rare events, whereas FOOP has only one.

Figures 5.14 and 5.15 graph the $\delta E^{*}$ and $\delta P^{*}$, respectively, of the linear friction system. These should be compared to Figures 4.18 and 4.20, the analogous results for FOOP. We 


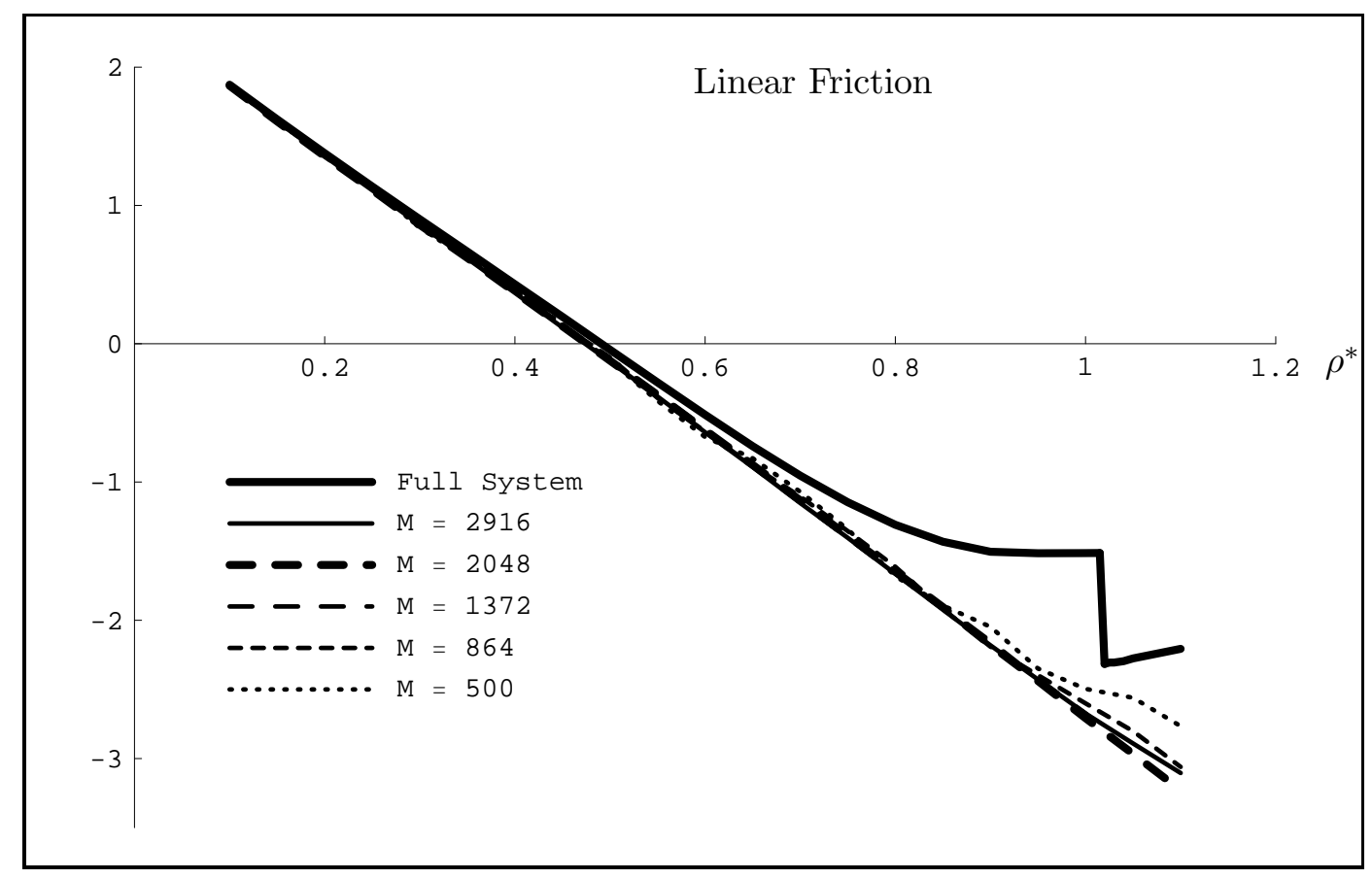

Figure 5.11: $E^{*} / N$ of the full system, and $\widehat{E}^{*}(M) / N$ calculated via the linear friction system for various $M$ values.

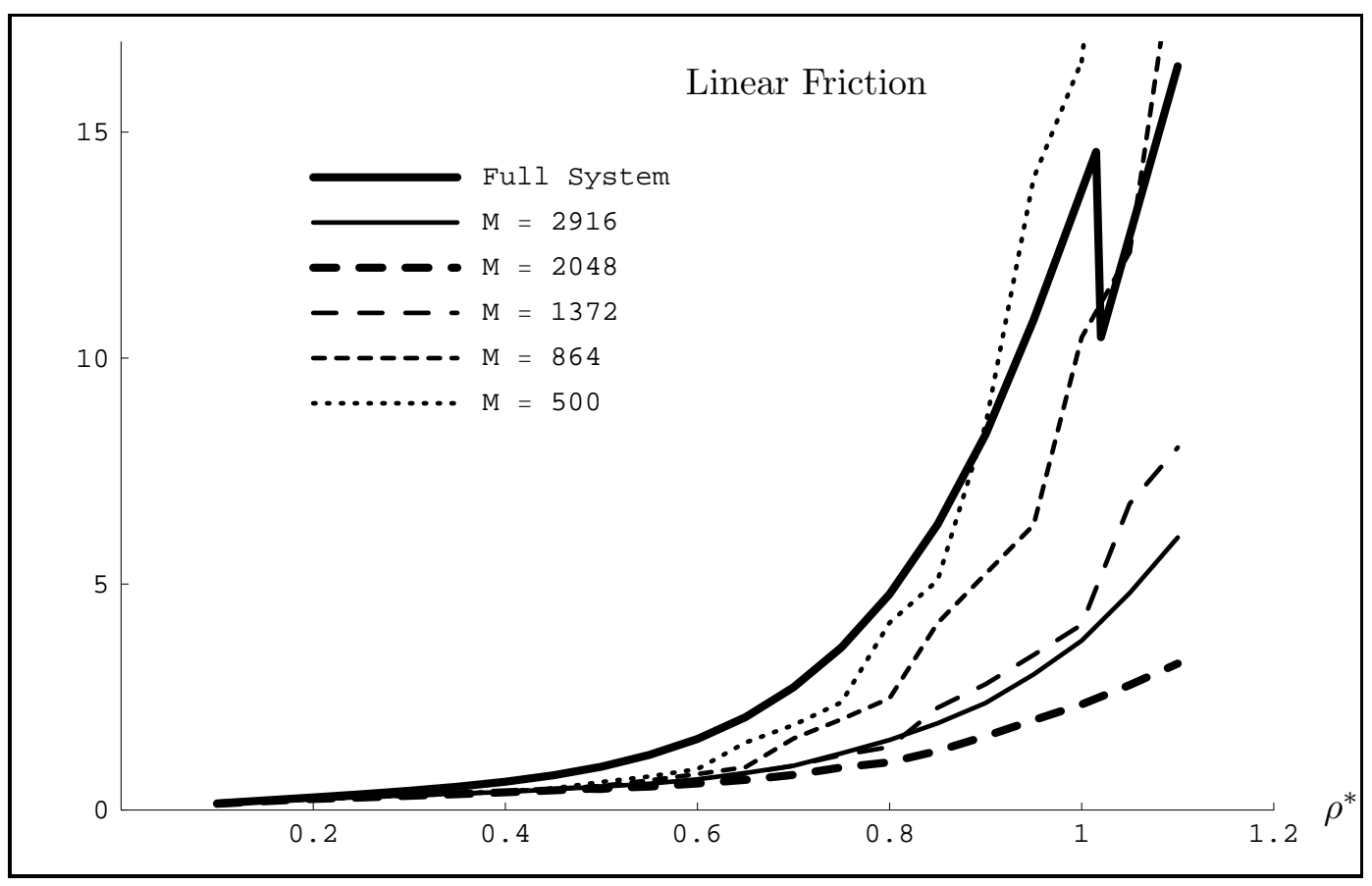

Figure 5.12: $P^{*}$ of the full system, and $\widehat{P}^{*}(M)$ calculated via the linear friction system for various $M$ values. 


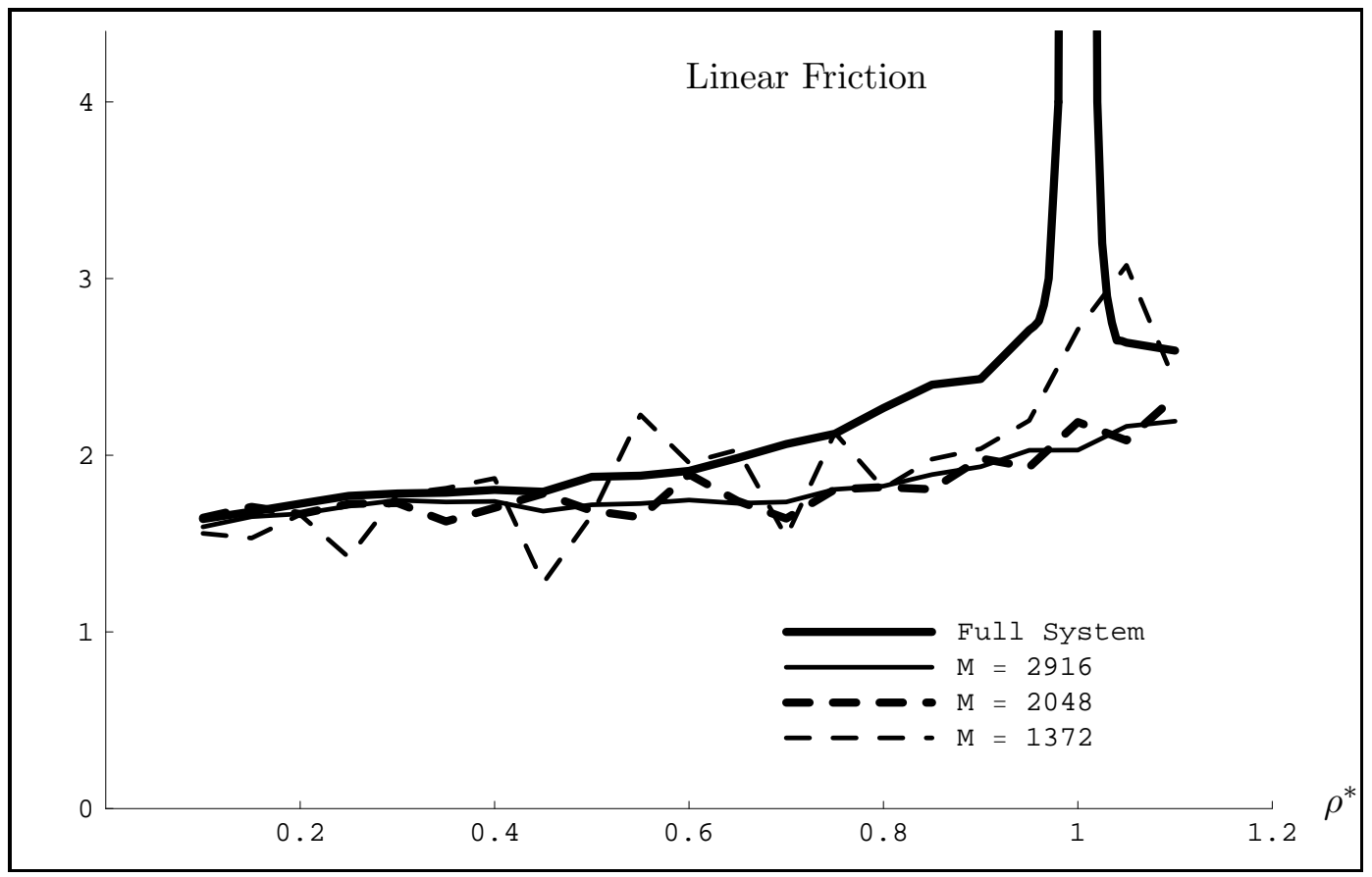

Figure 5.13: $C_{V}^{*} / N$ of the full system, and $\widehat{C}_{V}^{*}(M) / N$ calculated via the linear friction system for various $M$ values.

see that while the magnitudes of the errors under the LFA are somewhat larger than those of FOOP, as explained above, the qualitative behavior is the same: smaller errors at high and low values of $M$, with a maximum in the intermediate region. This serves to further confirm the validity of the formalism presented in Section 4.4, which predicts this effect.

\section{Autocorrelation Functions}

The velocity autocorrelation functions and shear stress autocorrelations for the linear friction system were calculated via the method derived in Section 4.3.2. Examples of the VAC and SSAC for the LFA system are shown in Figures 5.16 and 5.17, respectively. These should be compared to Figures 4.22 and 4.23 . Whereas for static averages the LFA performed slightly worse than FOOP, for autocorrelation functions the results are clearly far superior. Particularly striking are the low and moderate density VAC's, which lie essentially on top of the exact full system result for all values of $M$. Even for high density, the LFA curves are much closer to the truth than those obtained from FOOP, although the linear friction model is incapable of capturing the complex oscillatory behavior of the high-density autocorrelation function. This is due to the breakdown of the short-memory assumption embodied in equations (5.3) and (5.4).

The linear friction system (5.62) qualitatively resembles the Langevin equation in the form (5.14), which was originally proposed as a way to describe the behavior of systems such as a massive Brownian particle under constant bombardment by the much lighter molecules of the fluid that surrounds it. In that case, one would expect a large separation of time scales between the slow motion of the Brownian particle and the rapid motion of the particles striking it, and a short-memory approximation would be appropriate. What we 


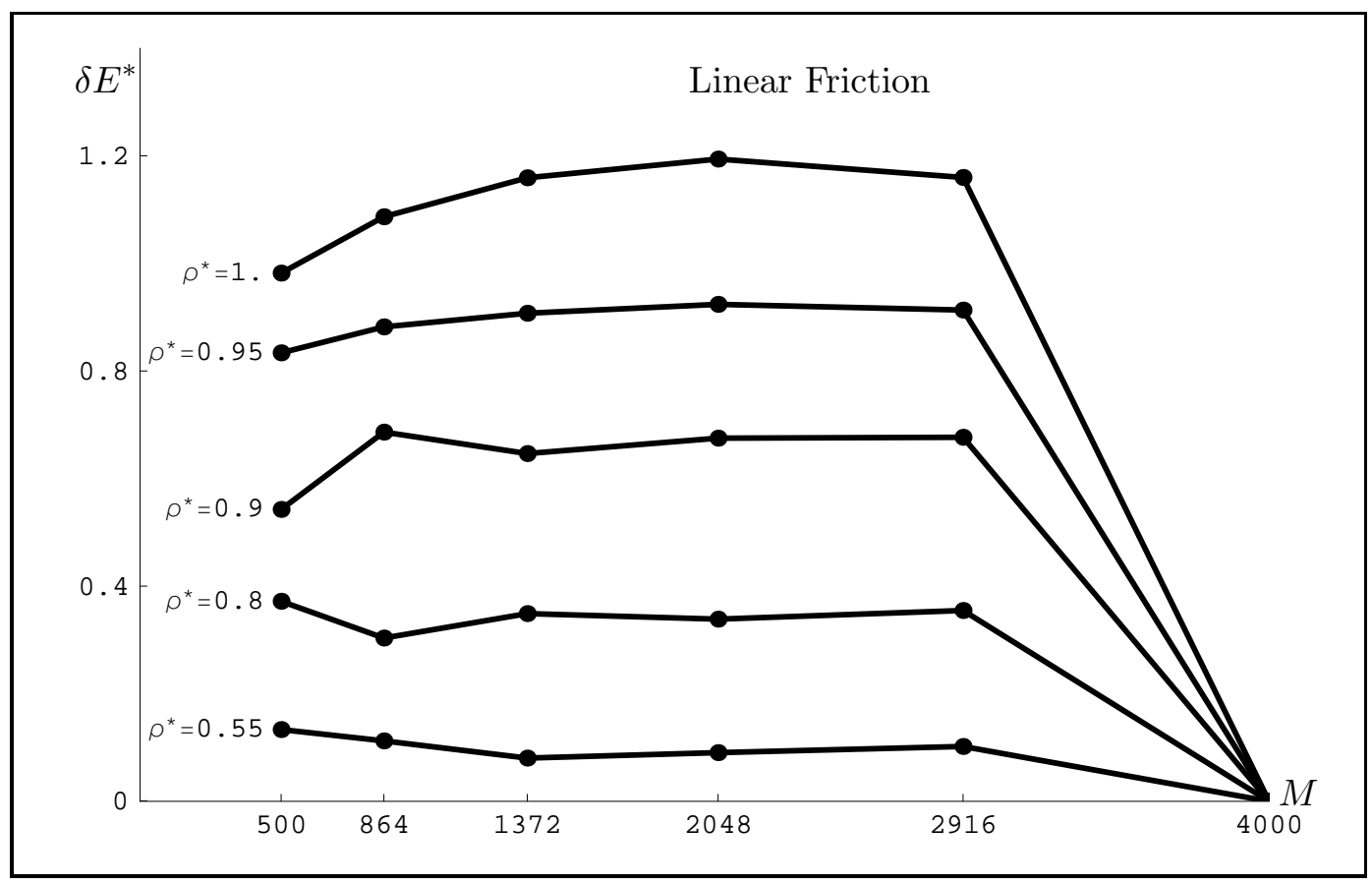

Figure 5.14: $\delta E^{*}$ of the linear friction system as a function of $M$, for several densities.

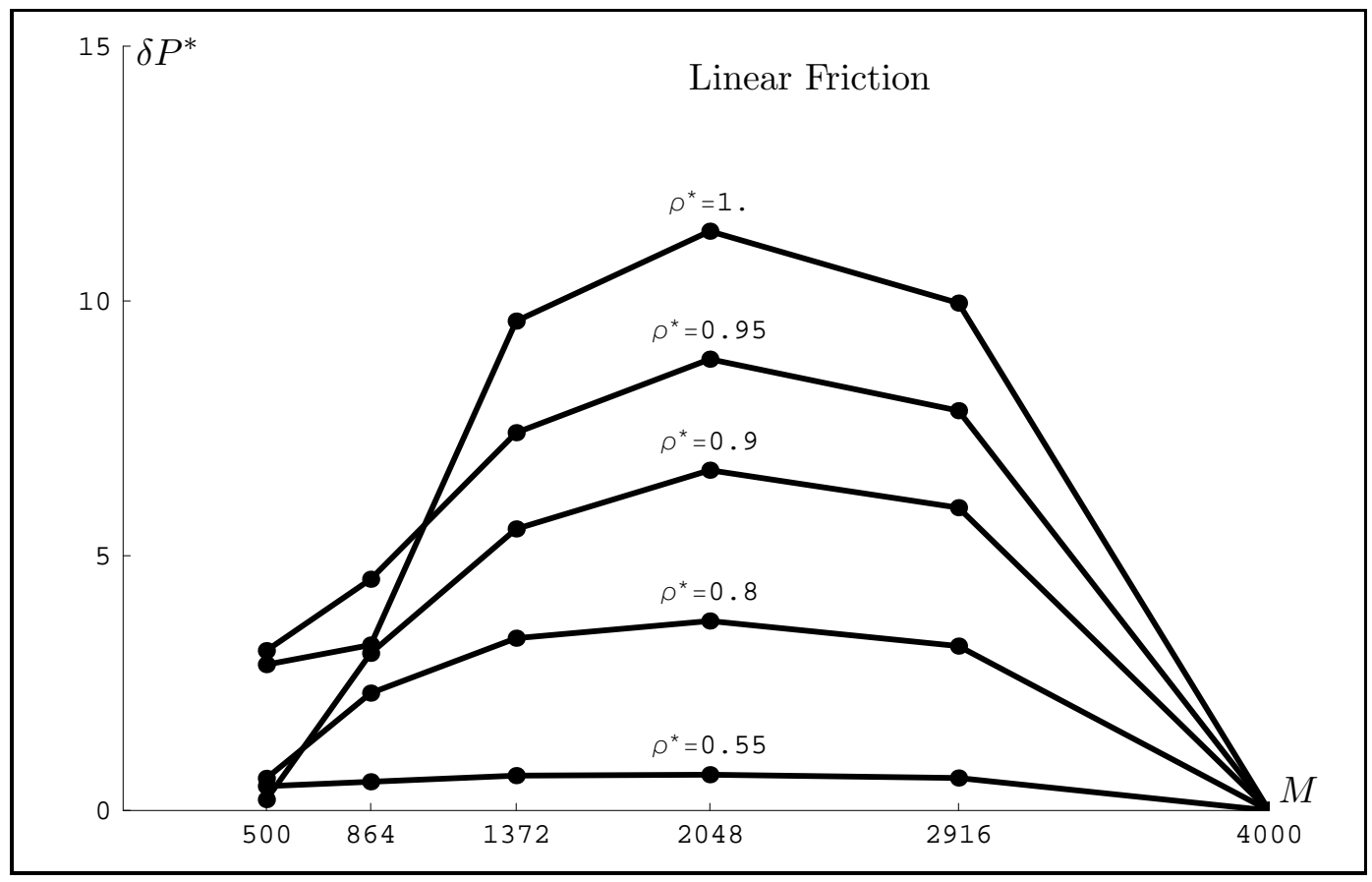

Figure 5.15: $\delta P^{*}$ of the linear friction system as a function of $M$, for several densities. 
are considering, on the other hand, is a simple fluid of identical-mass molecules, in which the time scale of the motion of any given particle is the same as the time scale of those colliding with it. As long as the density is low, subsequent collisions are reasonably uncorrelated, and a short-memory approximation is plausible. At high densities, however, each particle is trapped in an effective potential well due to the combined influence of its neighbors, and its motion becomes highly self-correlated for long periods of time. In this case, the support of the memory kernel (5.2) should extend far back in time, and the approximations (5.3) and (5.4) become untenable.

As in Chapter 4, the shear stress autocorrelation functions of this reduced model are subject to a significant degree of noise. Nevertheless, we can see that the decay rates of the LFA SSAC's are an improvment over the results of Chapter 4, especially at low densities, although there is an increased deviation in the value of $C_{\widehat{\Xi} \widehat{\Xi}}(0)$, for reasons explained above.

The results of this section show that even the rather crude approximation we have made to the memory and random forcing terms of optimal prediction can lead to a remarkable improvement in the degree to which the reduced system captures the dyamical properties of the underlying full system. 


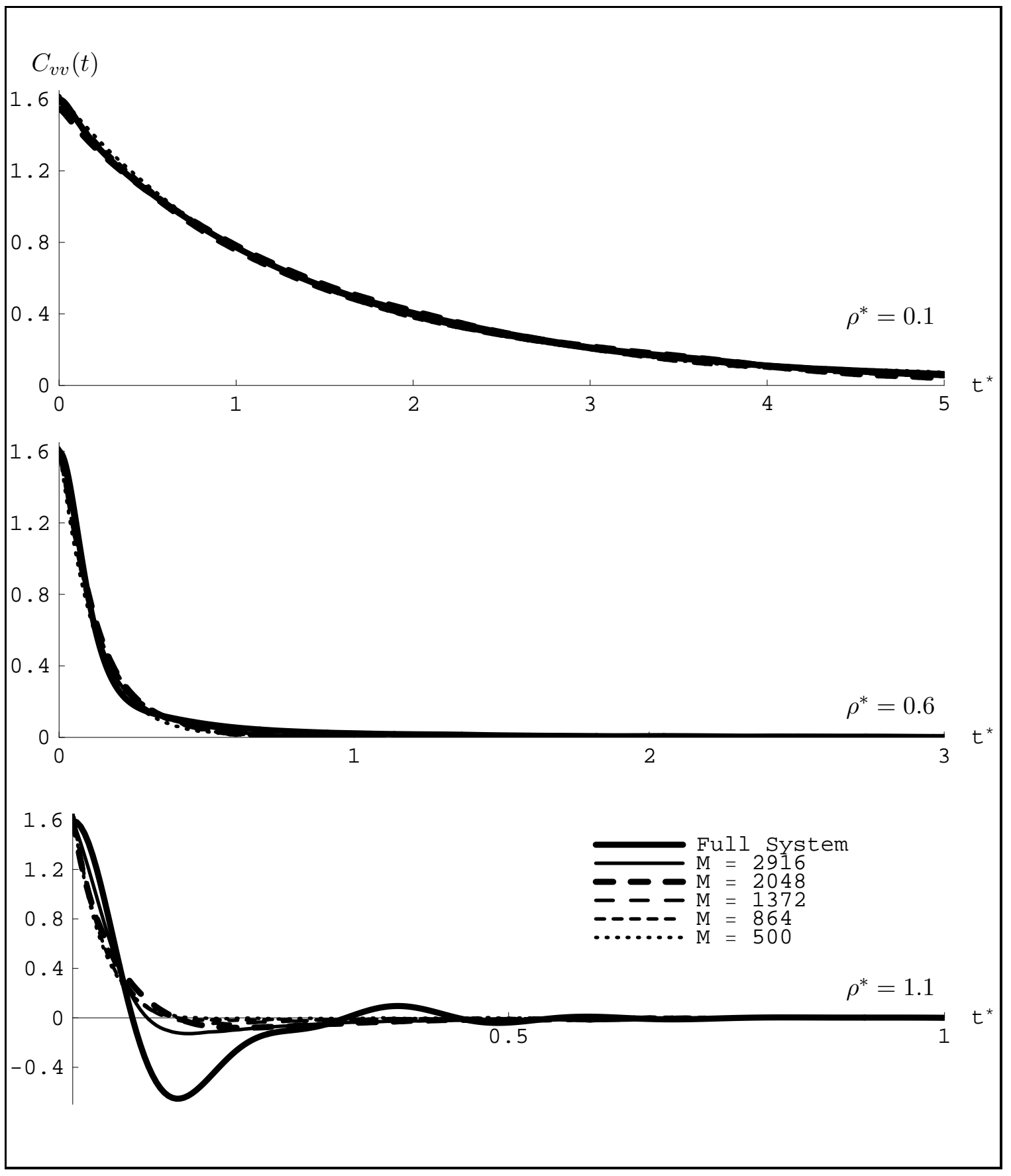

Figure 5.16: Velocity autocorrelation functions calculated via the full system and via linear friction, for various values of $M$, and for several densities. 


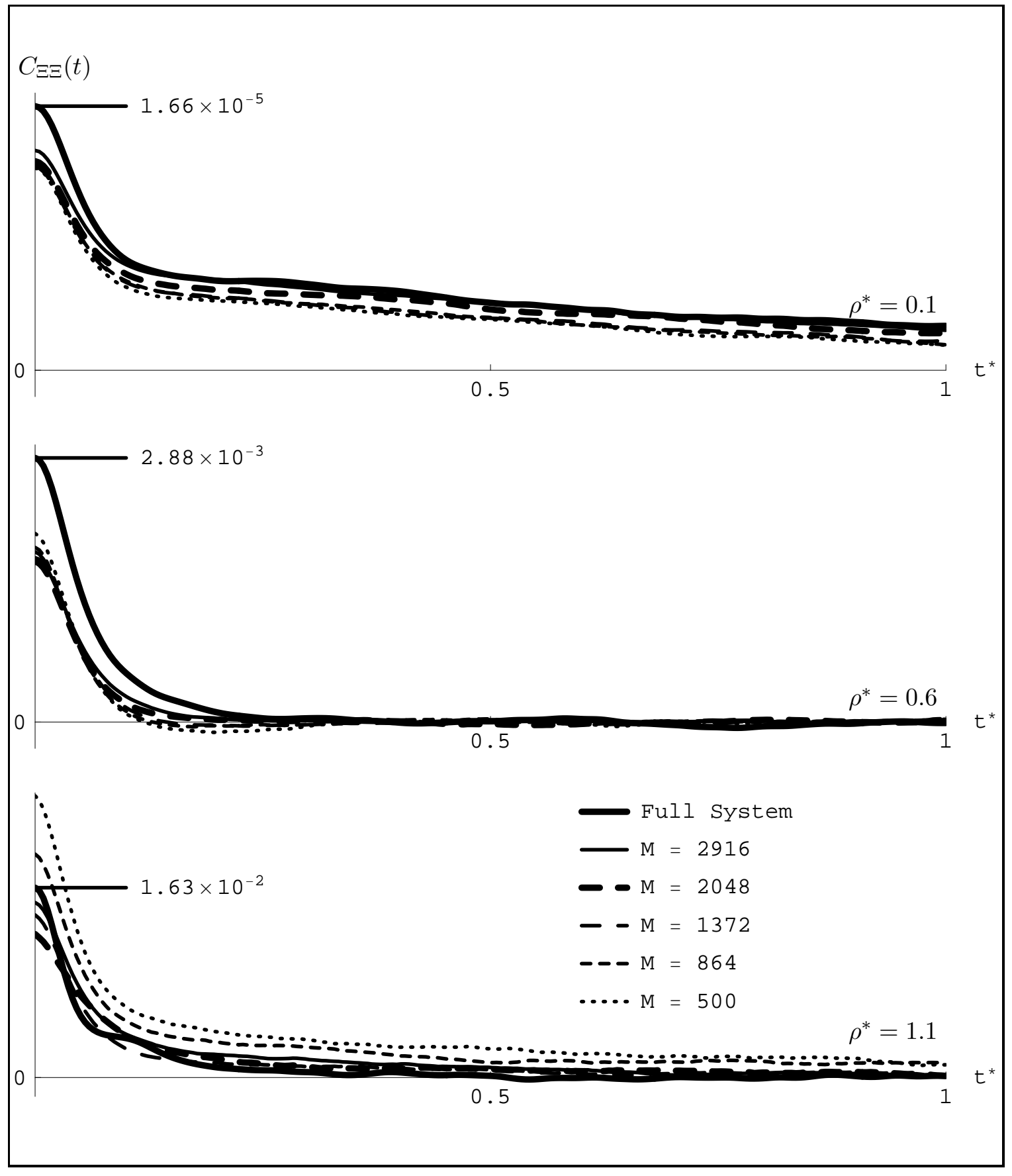

Figure 5.17: Shear stress autocorrelation functions calculated via the full system and via linear friction, for various values of $M$, and for several densities. The values of $C_{\Xi \Xi}(0)$ for the full system are labeled for reference. 


\section{Chapter 6}

\section{Conclusions}

The goal of this thesis was to construct a formalism for applying OP to the problem of the MD of an $N$-particle system, by devising a smaller system of $M$ particles from which the properties of the original system could be recovered. In this chapter, we will summarize the progress that has been made towards this goal, and suggest several possible future avenues of research.

In Chapter 2, after presenting some necessary background from probability theory, we described the foundations of OP, which follow from a particular implementation of the Mori-Zwanzig formalism. We then established some general notational conventions for the application of OP to Hamiltonian systems, which were used throughout this thesis.

In Chapter 3 we described a very general system of $N$ classical, identical, point particles. The equations of OP from Chapter 2 were then cast into a form more easily applicable to the set of Hamiltonian ODE's that governs this system. We then outlined the standard techniques of MD simulation. It is important to note that the form of MD considered in this thesis is of the simplest and oldest type, and that many variants and additions have been formulated over the years. Nevertheless, these methods form the backbone of most modern MD simulation schemes. Thus, the main results of this thesis can potentially be applied to many disparate areas of atomistic modeling.

The remainder of the thesis concerned the derivation and application of various approximations to the full equations of motion from Chapter 3. Chapter 4 described first order optimal prediction (FOOP), in which the exact integro-differential equations of motion for the reduced system are approximated by an effective dynamics governed by a renormalized Hamiltonian $\mathscr{H}$. Our investigation into the form of $\mathscr{H}$ allowed us to make a number of new connections between OP and several classical results from the microscopic theory of fluids. In particular, the $M$-particle distribution function $g^{(M)}$ arose naturally when considering the nature of the renormalized potential $\widehat{\phi}$. We were therefore able to take advantage of the large literature regarding $g^{(M)}$. For example, we made use of the low density expansion for the $n$-particle potential of mean force, as well as various related convergence properties. These results are not typically seen applied in a dynamical context, as has been done here.

A large part of Chapter 4 was spent outlining two of the main results of this thesis. In Section 4.3, we proved that if the exact renormalized potential is used, many of the static properties of the original $N$-particle system can be extracted from averages of dynamical variables under the reduced system. This involved the use of scaling expressions which are rigorously constrained to reproduce the analogous quantities in the full system.

In reality, however, we were forced to use a pairwise approximation to the exact ex- 
pression for $\widehat{\phi}$. The second major result of Chapter 4 was the development, in Section 4.4, of a formalism that yields a qualitative explanation for when and how this approximation will fail. Although not quantitative, this formalism makes the nonintuitive prediction that, after a certain point, reducing the number of resolved particles in the approximate system can actually improve the accuracy of the scaling expressions described above.

The numerical results of Chapter 4 confirmed that FOOP can accurately reproduce the energy and pressure of the full system at low-to-moderate densities, while requiring a fraction of the computer time. For example, the $M=500$ FOOP system predicted the correct energy and pressure for $\rho^{*} \leq 0.75$ and $\rho^{*} \leq 0.60$, respectively, to within an average error of $\sim \% 5$. On the other hand, it required, on average, $\sim 1 / 35^{\text {th }}$ as much runtime as the full system to achieve this result. Of nearly equal importance, however, was the behavior of FOOP in the region where it failed. An analysis of the high-density errors clearly confirmed the surprising prediction of Section 4.4, in that the deviations between the FOOP estimates and the exact values of the energy and pressure decreased with decreasing $M$ for $M \leq 2048$.

This has profound implications for the scalability of FOOP to larger system sizes. For example, with modern computers, MD simulations with $N \sim 1,000,000$ are commonplace. If we were to take this as our full system, it would not be unreasonable to consider a reduced FOOP system with $M \sim 5000$. As mentioned in Section 4.4, this is at least one order of magnitude higher than the number of particles required to avoid small system-size effects. The only significant remaining source of error would then be associated with making the pairwise approximation to the renormalized potential. However, for these choices of $N$ and $M$ we have $M / N \sim 0.005$. Results such as those shown in Figure 4.20 strongly suggest that in that case the error would drop to approximately zero. Meanwhile, this reduced system would require only a small fraction of the runtime.

In Chapter 5, we improved upon FOOP's treatment of dynamical quantities, such as autocorrelation functions, by making a short-memory approximation, in which the integral memory and random forcing terms were replaced by a linear friction and white noise, respectively. The form of the friction term was derived directly from the equations for the memory kernel, whereas the statistics of the forcing were deduced independently, by examining the Fokker-Planck equation for the distribution of the $2 M$ positions and momenta. We then proved that the resulting approximate system necessarily equilibrates to the renormalized canonical distribution, and described the similarities and differences between this linear friction approximation (LFA) and another coarse-graining technique known as Dissipative Particle Dynamics. The results of simulating the LFA system via a numerical method derived from the velocity Verlet scheme of MD showed that it is comparable to FOOP in terms of calculating static properties, but far better when it comes to measuring correlation functions. However, like FOOP, the LFA works poorly at high densities for the $M$ values considered in this thesis.

These two approximations, FOOP and the LFA, can serve as a basis for further developments. One way to better capture the static properties of high density systems such as solids or dense liquids would be to include some approximation to the three body potential. In terms of evaluating dynamical quantities, it would also be useful to construct a form for the memory kernel that allows finite-time memory effects, which would in turn transform the random forcing term into a colored noise process with a known autocorrelation function. Furthermore, there are many other possible forms for the memory kernel that arise by considering alternate projection operators in its derivation, some of which may lead to better results. These have yet to be explored. 
It would be interesting to move beyond the determination of simple fluid properties, and into the realm of everyday MD applications. For instance, one might extend these methods to polyatomic molecules. Systems reduction for complex molecules would probably take a different form than that introduced here. For example, instead of decreasing the overall number of particles, one may choose to average over the internal degrees of freedom, and thus reduce polyatomic molecules to monatomic particles. This would yield a different type of renormalized potential, with different behavior. Another area that might benefit from these techniques is the simulation of biological macromolecules, such as proteins. In a typical single-protein simulation, a significant fraction of the computational time is spent modeling the behavior of solvent-solvent and solvent-protein interactions. Since the properties of interest are those of the protein itself, and the solvent is often represented as a simple, one-component fluid, we can imagine applying OP to the solvent, while the protein is left to evolve according to its full equations of motion.

Another setting in which these methods have yet to be explored is the study of nonequilibrium situations, in particular the determination of the mesoscopic flow of a fluid by MD. Since the formalism of this thesis uses the canonical distribution, which precludes large-scale flow, a new measure on the space of initial conditions appropriate to the nonequilibrium state would need to be constructed. This opens up the interesting possiblility of entire families of variations on optimal prediction, each predicated on the use of different nonequilibrium ensembles.

One point that should be stressed, however, is the fact that the techniques outlined in this thesis do not apply to systems that interact via the Coulomb potential, such as plasmas. A long-range bare potential would necessarily invalidate the diagrammatic expansion of Section 4.2.1, since the integrals to which the diagrams correspond would no longer converge. Furthermore, the form of the renormalized potential would depend strongly on the shape and size of the domain, as well as on position, i.e. spatial isotropy would no longer hold. This set of complications indicates that our formalism would need to be radically altered in order to accomodate the presence of ions or other charged particles. Furthermore, any discussion of plasmas or similar systems would certainly need to include the effects of external fields, which have been neglected in this thesis. This would require an additional term in the Hamiltonian of the form $\sum_{i} U_{1}\left(q_{i}\right)$, where $U_{1}$ is the one-particle potential associate with the external field. On this point, the outlook is more optimistic, since all that needs to be done is to associate with each particle a weight function $e^{-\beta U_{1}\left(q_{i}\right)}$, so that every integral of the form $\int d q_{i}(\cdots)$ becomes $\int d q_{i} e^{-\beta U_{1}\left(q_{i}\right)}(\cdots)$. If the length scale on which the external field varies is short compared to microscopic scales, then the only modification that would need to be made would be to include the parametric dependence of the renormalized potential and friction matrices upon the local values and gradients of the external potential. 


\section{Bibliography}

[1] B. Alder and T. Wainwright. Phase transition for a hard sphere system.

J. Chem. Phys., 27:1208-1209, 1957.

[2] B. Alder and T. Wainwright. Enhancement of diffusion by vortex-like motion of classical hard particles. Proceedings of the International Conference on Statistical Mechanics, Kyoto 1968. J. Phys. Soc. Japan Suppl., 26:267-269, 1969.

[3] M. Allen and D. Tildesley. Computer Simulation of Liquids. Oxford Science Publications, 1987.

[4] H. Andersen. Molecular dynamics at constant pressure and/or temperature. J. Chem. Phys., 72:2384-2393, 1980.

[5] A. Buckingham and D. Dunmur. Kerr effect in inert gases and sulphur hexaflouride. Trans. Faraday Soc., 64:1776-1783, 1968.

[6] A. Chorin. Stochastic methods in applied mathematics and physics (Lecture Notes). http://math.berkeley.edu/ ${ }^{\text {chorin/stinis2.ps }}$

[7] A. Chorin, O. Hald, and R. Kupferman. Optimal prediction and the Mori-Zwanzig representation of irreversible processes, Proc. Natl. Acad. Sci. USA. 97(7):2968-2973, 2000 .

[8] A. Chorin, O. Hald, and R. Kupferman. Non-Markovian optimal prediction. Monte Carlo Methods Appl., 7(1-2):99-109, 2001. Monte Carlo and probabilistic methods for partial differential equations (Monte Carlo, 2000).

[9] A. Chorin, O. Hald, and R. Kupferman. Optimal prediction with memory. Phys. D, 166(3-4):239-257, 2002.

[10] A. Chorin, A. Kast, and R. Kupferman. Optimal prediction of underresolved dynamics. Proc. Nat. Acad. Sci. USA, 95(8):4094-4098, 1998.

[11] A. Chorin, A. Kast, and R. Kupferman. On the prediction of large-scale dynamics using underresolved computations. In Nonlinear Partial Differential Equations (Evanston, IL, 1998), pages 53-75. Amer. Math. Soc., Providence, RI, 1999.

[12] A. Chorin, A. Kast, and R. Kupferman. Unresolved computation and optimal prediction. Comm. Pure Appl. Math., 52(10):1231-1254, 1999. 
[13] E. D'Azevedo, C. Romine, and D. Walker. Shared-memory emulation enables billionatom molecular dynamics simulation, in Applications on Advanced Architecture Computers, G. Astfalk, ed., SIAM, Philadelphia, 1996.

[14] F. Dyson. The radiation theories of Tomonaga, Schwinger, and Feynman. Phys. Rev., 75(3):486-502, 1949.

[15] W. E and Z. Huang. Matching conditions in atomistic-continuum modeling of materials. Phys. Rev. Lett., 87(13):135501, 2001.

[16] P. Español and P. Warren. Statistical Mechanics of dissipative particle dynamics. Europhys. Lett., 30(4):191-196, 1995.

[17] D. Evans and G. Morriss. Statistical Mechanics of Nonequilibrium Liquids. Academic Press, London, 1990.

[18] E. Flekkøy and P. Coveney. From molecular dynamics to dissipative particle dynamics. Phys. Rev. Lett., 83(9):1775-1778, 1999.

[19] D. Forster. Hydrodynamic Fluctuations, Broken Symmetry, and Correlation Functions. Benjamin, Reading, Massachussets, 1975.

[20] D. Givon, O. Hald, and R. Kupferman. Existence proof for orthogonal dynamics and the Mori-Zwanzig formalism. Israeli J. Math., 2004 (in press).

[21] A. Greenfield, J. Wellendorf, and N. Wiser. X-ray determination of the static structure factor of liquid Na and K. Phys. Rev. A, 4(4):1607-1616, 1971.

[22] J. Groeneveld. Estimation Methods for Mayer Graphical Expansions. Doctoral Thesis, University of Amsterdam, Netherlands, 1967.

[23] J. Hansen and I. McDonald. Theory of Simple Liquids. Academic Press, London, 1986.

[24] P. Hohenberg and B. Halperin. Theory of dynamic critical phenomena. Rev. Mod. Phys., 49(3):435-479, 1977.

[25] P. Hoogerbrugge and J. Koelman. Simulating microscopic hydrodynamic phenomena with dissipative particle dynamics. Europhys. Lett., 19(3):155-160, 1992.

[26] W. Hoover. Canonical Dynamics: Equilibrium phase-space distributions. Phys. Rev. A, 31(3):1695-1697, 1985.

[27] E. Ingerman. Modelling the Loss of Information in Optimal Prediction. PhD Thesis, University of California at Berkeley, Berkeley, CA 94720, 2003.

[28] C. Johnson. Numerical Solution of Partial Differential Equations by the Finite Element Method. Cambridge University Press, Cambridge, 1987.

[29] J. Kirkwood. Statistical mechanics of fluid mixtures. J. Chem. Phys., 3:300-313, 1935.

[30] C. Kittel. Introduction to Solid State Physics, Seventh Edition. John Wiley \& Sons, Inc., New York, 1996. 
[31] A. Ladd. Short-time motion of colloidal particles: Numerical simulation via fluctuating Lattice-Boltzmann Equation. Phy. Rev. Lett., 70(9):1339-1342, 1993.

[32] R. Larsen and M. Marx. An Introduction to Mathematical Statistics and Its Applications. Prentice-Hall, New Jersey, 1986.

[33] J. Lebowitz. Statistical Mechanics - A Review of Selected Rigorous Results. Ann. Rev. Phys. Chem., 19:389-418, 1968.

[34] J. Lebowitz and O. Penrose. Convergence of Virial Expansions. J. Math. Phys., 5(7):841-847, 1964.

[35] J. Lennard-Jones. Wave functions of many-electron atoms. Proc. Cambridge Phil. Soc., 27:469-480, 1931

[36] D. Levesque and L. Verlet. Computer "experiments" on classical fluids. III. Timedependent self-correlation functions. Phys. Rev. A, 2(6):2514-2528, 1970.

[37] D. Levesque, L. Verlet, and J. Kürkijarvi. Computer "experiments" on classical fluids. IV. Transport properties and time-correlation functions of the Lennard-Jones fluid near its triple point. Phys. Rev. A, 7(5):1690-1700, 1973.

[38] S. Lin, M. Blanco, W. Goddard. The two-phase model for calculating thermodynamic properties of liquids from molecular dynamics: Validation of the phase diagram for Lennard-Jones fluids. J. Chem. Phys., 119(22):11792-11805, 2003.

[39] S. Lovesey. Single-particle motion in classical monatomic liquids. J. Phys. C: Solid State Phys., 6:1856-1862, 1973.

[40] C. Marsh. Theoretical Aspects of Dissipative Particle Dynamics. PhD Thesis, University of Oxford, Oxford, U.K., 1998.

[41] J. Mayer and M. Mayer. Statistical Mechanics. Wiley, New York, 1940.

[42] J. Mayer and E. Montroll. Statistical Mechanics of Imperfect Gases. J. Chem. Phys., 9:626-637, 1941.

[43] N. Metropolis, A. Rosenbluth, M. Rosenbluth, A. Teller, and E. Teller. Equation of state calculations by fast computing machines. J. Chem. Phys., 21:1087-1092, 1953.

[44] H. Mori. Transport, collective motion, and Brownian motion. Prog. Theor. Phys., 33(3):423-455, 1965.

[45] P. Morse. Diatomic Molecules according to the wave mechanics. II. Vibrational levels. Phys. Rev., 34(1):57-64, 1929.

[46] M. Murat and K. Kremer. From many monomers to many polymers: Soft ellipsoid model for polymer melts and mixtures. J. Chem. Phys., 108(10):4340-4348, 1998.

[47] S. Nosé. Constant temperature molecular dynamics methods. Prog. Theor. Phys. Supplement, 103:1-46, 1991.

[48] R. Pathria. Statistical Mechanics, Second Edition. Butterworth-Heinemann, Oxford, 1996. 
[49] A. Pazy. Semigroups of Linear Operators and Applications to Partial Differential Equations. Springer-Verlag, New York, 1983.

[50] J. Potoff and A. Panagiotopoulos. Critical point and phase behaviour of the pure fluids and a Lennard-Jones mixture. J. Chem. Phys., 109:14-20, 1998.

[51] W. Press, A. Teukolsky, W. Vetterling, and B. Flannery. Numerical Recipes in C. Cambridge University Press, Cambridge, 1992.

[52] A. Rahman. Correlations in the motion of atoms in liquid argon. Phys. Rev., 136(2A):405-411, 1964.

[53] D. Reichman and E. Rabani. Self-consistent mode-coupling theory for self-diffusion in quantum liquids. Phys. Rev. Lett., 87(26):265702, 2001.

[54] D. Ruelle. Classical Mechanics of a System of Particles Helv. Phys. Acta., 36:183-197, 1963.

[55] D. Ruelle. Statistical Mechanics: Rigorous Results. Benjamin, Reading, 1969.

[56] Ch. Schütte, A. Fischer, W. Huisinga, and P. Deuflhard. A direct approach to conformational dynamics based on hybrid Monte Carlo. J. Comp. Phys., 151:146-168, 1999.

[57] B. Seibold. Optimal Prediction in Molecular Dynamics. Diploma Thesis, University of Kaiserslautern, Kaiserslautern, Germany, 2003.

[58] B. Seibold. Optimal prediction in molecular dynamics. Monte Carlo Methods and Appl., 10(1):25-50, 2004.

[59] L. Sjögren and A. Sjölander. Kinetic theory of self-motion in monatomic liquids. J. Phys. C: Solid State Phys., 12:4369-4392, 1979.

[60] R. Skeel, G. Zhang, and T. Schlick. A family of symplectic integrators: stability, accuracy, and molecular dynamics applications. SIAM J. Sci. Comput., 18(1):203-222, 1997.

[61] A. Sveshnikov. Problems in Probability Theory, Mathematical Statistics, and the Theory of Random Functions. Dover Publications, New York, 1978.

[62] W. Swope, H. Anderson, P. Berens, and K. Wilson. A computer simulation method for the calculation of equilibrium constants for the formation of physical clusters of molecules: Application to small water clusters. J. Chem. Phys., 76(1):637-649, 1982.

[63] K. Theodoropoulos, Y.-H. Qian, and I. Kevrekidis. "Coarse" stability and bifurcation analysis using timesteppers: a reaction-diffusion example. Proc. Natl. Acad. Sci., 97(18):9840-9843, 2000.

[64] S. Thompson. Use of neighbour lists in molecular dynamics. CCP5 Newsletter, No. 8, 1983.

[65] L. Verlet. Computer "experiments" on classical fluids. I. Thermodynamical properties of Lennard-Jones molecules. Phys. Rev., 159(1):98-103, 1967. 
[66] L. Verlet. Computer "experiments" on classical fluids. II. Equilibrium correlation functions. Phys. Rev., 165(1):201-214, 1968.

[67] K. Zahn, G. Maret, C. Ruß, and H. von Grünberg. Three-particle correlations in simple liquids. Phys. Rev. Lett., 91(11):5502-5505, 2003.

[68] R. Zwanzig. Ensemble method in the theory of irreversibility. J. Chem. Phys., 33(5):1338-1341, 1960.

[69] R. Zwanzig. Memory effects in irreversible thermodynamics. Phys. Rev., 124(4):983-992, 1961. 


\section{Appendix A}

\section{Evaluation of the Shear Stress Autocorrelation Function at $t=0$}

The shear stress autocorrelation function is

$$
C_{\Xi \Xi}(t)=\left\langle\Xi_{\mu \nu}(t) \Xi_{\mu \nu}(0)\right\rangle_{C} \quad \quad \mu \neq \nu,
$$

where

$$
\Xi_{\mu \nu}=\frac{1}{V} \sum_{i=1}^{N}\left(\frac{1}{m} p_{i \mu} p_{i \nu}-q_{i \mu} \nabla_{q_{i \nu}} \phi(q)\right)
$$

is an off-diagonal component of the stress tensor. Our goal is to calculate $C_{\Xi \Xi}(0)$. Since any $\mu \nu$ pair will yield the same result, we will choose $\mu=1$ and $\nu=2$ for simplicity. Then

$$
\begin{aligned}
& C_{\Xi \Xi}(0)=\frac{1}{V^{2}}\left\langle\sum_{i=1}^{N}\left(\frac{1}{m} p_{i 1} p_{i 2}-q_{i 1} \nabla_{q_{i 2}} \phi(q)\right) \sum_{j=1}^{N}\left(\frac{1}{m} p_{j 1} p_{j 2}-q_{j 1} \nabla_{q_{j 2}} \phi(q)\right)\right\rangle_{C} \\
& =\frac{1}{V^{2}} \sum_{i, j=1}^{N}\{\underbrace{\left\langle\frac{1}{m^{2}} p_{i 1} p_{i 2} p_{j 1} p_{j 2}\right\rangle_{C}}_{=\left(k_{B} T\right)^{2} \delta_{i j}}-\underbrace{\left\langle\frac{1}{m} p_{i 1} p_{i 2} q_{j 1} \nabla_{q_{j 2}} \phi\right\rangle_{C}}_{=0} \\
& -\underbrace{\left\langle\frac{1}{m} p_{j 1} p_{j 2} q_{i 1} \nabla_{q_{i 2}} \phi\right\rangle_{C}}_{=0}+\left\langle\left(q_{i 1} \nabla_{q_{i 2}} \phi\right)\left(q_{j 1} \nabla_{q_{j 2}} \phi\right)\right\rangle_{C}\} \\
& =\frac{1}{V} \rho\left(k_{B} T\right)^{2}+\underbrace{\frac{1}{V^{2}} \sum_{i, j=1}^{N}\left\langle\left(q_{i 1} \nabla_{q_{i 2}} \phi\right)\left(q_{j 1} \nabla_{q_{j 2}} \phi\right)\right\rangle_{C}}_{\equiv \Lambda},
\end{aligned}
$$

where in the second line we have made repeated use of the fact that, under the canonical distribution, $\left\langle p_{i \mu} p_{j \mu}\right\rangle_{C}=m k_{B} T \delta_{i j} \delta_{\mu \nu}$. This follows because the different components of the momentum for each of the $N$ particles are independently distributed according to a Maxwellian distribution. Let us concentrate on the second term (which we have labeled 
" $\Lambda$ " for ease of reference):

$$
\Lambda=\frac{1}{V^{2}} \sum_{i, j=1}^{N} \frac{1}{Z_{c}} \int d q\left(q_{i 1} \nabla_{q_{i 2}} \phi\right)\left(q_{j 1} \nabla_{q_{j 2}} \phi\right) e^{-\beta \phi} .
$$

Here $Z_{c} \equiv \int d q e^{-\beta \phi}$ is known as the configurational part of the partition function. We now make use of the fact that

$$
\left(q_{i 1} \nabla_{q_{i 2}} \phi\right) e^{-\beta \phi}=-k_{B} T \nabla_{q_{i 2}}\left(q_{i 1} e^{-\beta \phi}\right)
$$

and perform an integration by parts, to arrive at

$$
\Lambda=\frac{k_{B} T}{V^{2}} \sum_{i, j=1}^{N} \frac{1}{Z_{c}} \int d q q_{i 1} q_{j 1}\left(\nabla_{q_{i 2}} \nabla_{q_{j 2}} \phi\right) e^{-\beta \phi} .
$$

In the above expression, there are $N$ terms with $i=j$ (all of which are equal), and $N(N-1)$ terms with $i \neq j$ (all of which are equal). This allows us to write

$$
\Lambda=\frac{k_{B} T}{V^{2}} \frac{1}{Z_{c}}\left\{N \int d q q_{11}^{2}\left(\nabla_{q_{12}} \nabla_{q_{12}} \phi\right) e^{-\beta \phi}+N(N-1) \int d q q_{11} q_{21}\left(\nabla_{q_{12}} \nabla_{q_{22}} \phi\right) e^{-\beta \phi}\right\}
$$

Since

$$
\nabla_{q_{12}} \nabla_{q_{12}} \phi=\sum_{i=2}^{N} \nabla_{q_{12}} \nabla_{q_{12}} U_{1 i}
$$

and

$$
\nabla_{q_{12}} \nabla_{q_{22}} \phi=\nabla_{q_{12}} \nabla_{q_{22}} U_{12}=-\nabla_{q_{12}} \nabla_{q_{12}} U_{12}
$$

we can use indistinguishability of particles to obtain:

$$
\Lambda=\frac{k_{B} T}{V^{2}} \frac{N(N-1)}{Z_{c}} \int d q\left(q_{11}^{2}-q_{11} q_{21}\right)\left(\nabla_{q_{12}} \nabla_{q_{12}} U_{12}\right) e^{-\beta \phi} .
$$

This can be simplified further by realizing that $q_{11}^{2}-q_{11} q_{21}=\left(q_{11}-q_{21}\right)^{2}-\left(q_{21}^{2}-q_{11} q_{21}\right)$. By symmetry, the quantity $q_{21}^{2}-q_{11} q_{21}$ gives the same result when substituted for $q_{11}^{2}-q_{11} q_{21}$ in the above integral. Thus

$$
\Lambda=\frac{k_{B} T}{V^{2}} \frac{1}{Z_{c}} \frac{N(N-1)}{2} \int d q\left(q_{11}-q_{21}\right)^{2}\left(\nabla_{q_{12}} \nabla_{q_{12}} U_{12}\right) e^{-\beta \phi} .
$$

According to the definition of the radial distribution function [23],

$$
\frac{1}{Z_{c}} \int d q_{3} \ldots d q_{N} e^{-\beta \phi}=\rho^{2} \frac{1}{N(N-1)} g^{(2)}\left(\left|q_{1}-q_{2}\right|\right) .
$$

If we take advantage of this, and define a new variable $x=q_{1}-q_{2}$ with components $x_{1}, x_{2}$, etc, then $\Lambda$ simplifies to

$$
\Lambda=\frac{k_{B} T}{2 V} \rho^{2} \int d x x_{1}^{2}\left(\partial_{x_{2}}^{2} U(|x|)\right) g^{(2)}(|x|)
$$


Finally, we switch to spherical polar coordinates and perform the angular part of the resulting integral, which yields

$$
\Lambda=\frac{2 \pi}{15} \frac{k_{B} T}{V} \rho^{2} \int_{0}^{\infty} d r \frac{d}{d r}\left(r^{4} U^{\prime}(r)\right) g^{(2)}(r) .
$$

Our final expression for $C_{\Xi \Xi}(0)$ is therefore

$$
C_{\Xi \Xi}(0)=\frac{k_{B} T}{V}\left\{\rho k_{B} T+\frac{2 \pi}{15} \rho^{2} \int_{0}^{\infty} d r \frac{d}{d r}\left(r^{4} U^{\prime}(r)\right) g^{(2)}(r)\right\} .
$$

